\title{
Next Generation Nuclear Plant Methods Research and Development Technical Program Plan
}

September 2008

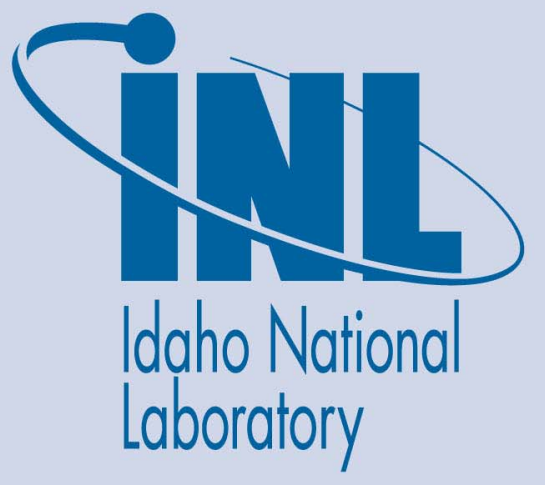

The INL is a U.S. Department of Energy National Laboratory operated by Battelle Energy Alliance 
INL/EXT-06-11804

PLN-2498

Rev. 1

\title{
Next Generation Nuclear Plant Methods Research and Development Technical Program Plan
}

September 2008

\author{
Idaho National Laboratory \\ Idaho Falls, Idaho 83415
}

http://www.inl.gov

Prepared for the

U.S. Department of Energy

Office of Nuclear Energy

Under DOE Idaho Operations Office

Contract DE-AC07-05ID14517 


\begin{tabular}{|c|c|c|c|}
\hline NEXT GENERATION NUCLEAR PLANT & Identifier: & PLN-2498 & \\
\hline METHODS RESEARCH AND & Revision: & 1 & \\
\hline $\begin{array}{l}\text { DEVELOPMENT TECHNICAL } \\
\text { PROGRAM PLAN }\end{array}$ & Effective Date: & $9 / 25 / 08$ & Page: ii of ii \\
\hline
\end{tabular}

\section{REVISION LOG}

\begin{tabular}{|c|c|c|c|}
\hline Rev. & Date & Affected Pages & Revision Description \\
\hline 1 & $9 / 25 / 08$ & Chapter 5 & $\begin{array}{l}\text { Chapter 5,"neutronics an Reactor Kinetics Analysis } \\
\text { Development" has been completely rewritten to } \\
\text { emphasive the needs for new or enhanced neutronic } \\
\text { methods such as PEBBED-THERMIX-COMBINE- } \\
\text { NEM and RELAP-CYNOD-COMBINE. }\end{array}$ \\
\hline 1 & $9 / 25 / 08$ & All & $\begin{array}{l}\text { Minor editorial and grammatical changes throughout } \\
\text { the document }\end{array}$ \\
\hline & & & \\
\hline & & & \\
\hline & & & \\
\hline & & & \\
\hline & & & \\
\hline & & & \\
\hline & & & \\
\hline & & & \\
\hline & & & \\
\hline & & & \\
\hline & & & \\
\hline
\end{tabular}




\begin{tabular}{|c|c|c|c|}
\hline $\begin{array}{c}\text { NEXT GENERATION NUCLEAR PLANT } \\
\text { METHODS RESEARCH AND } \\
\text { DEVELOPMENT TECHNICAL } \\
\text { PROGRAM PLAN }\end{array}$ & $\begin{array}{l}\text { Identifier: } \\
\text { Revision: } \\
\text { Effective Date: }\end{array}$ & $\begin{array}{l}\text { PLN-2498 } \\
125 \\
9 / 24 / 08\end{array}$ & Page: \\
\hline
\end{tabular}

\section{Next Generation Nuclear Plant Methods Research and Development Technical Program Plan}

PLN-2498

Revision 1

Approved by:
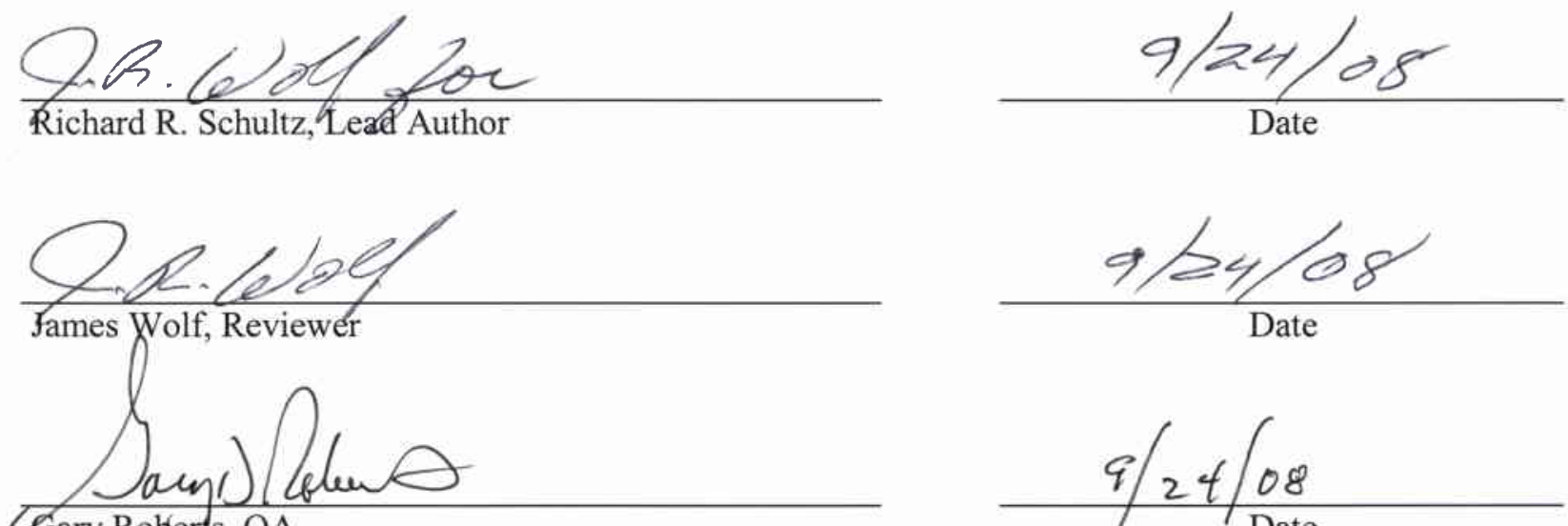

Gary Roberts, QA
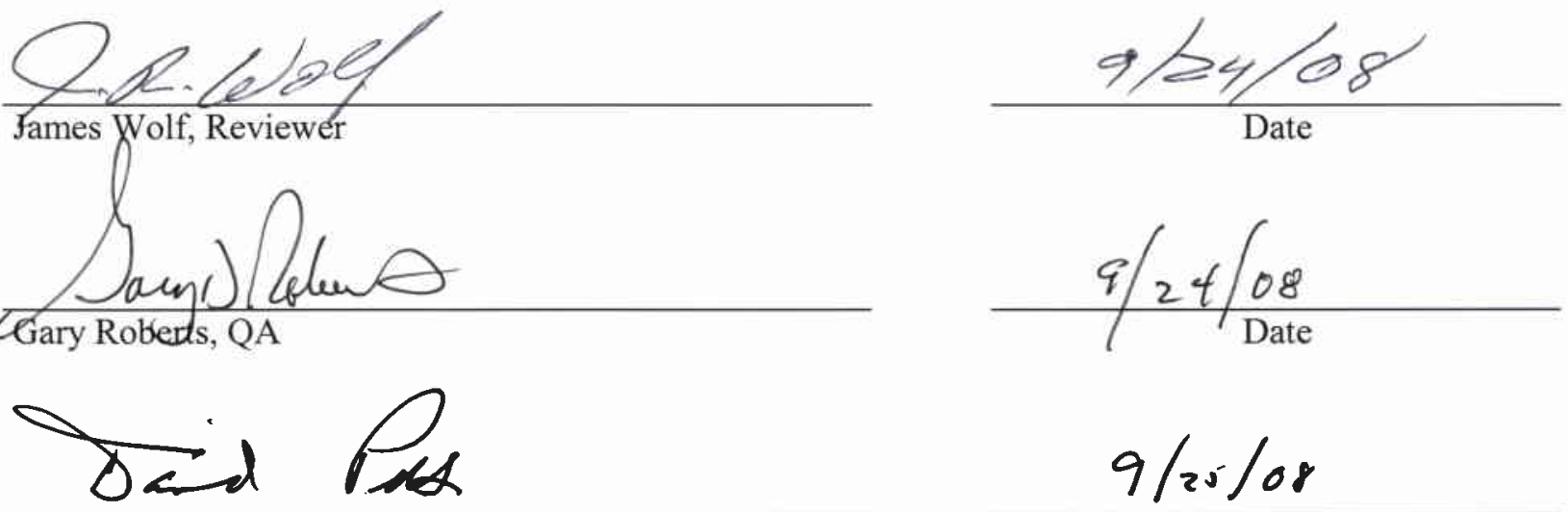

David Petti, TDO Project Director

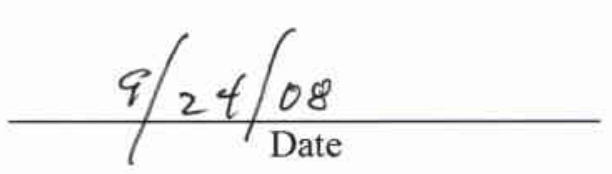

$9 / 25 / 08$ Date 


\begin{tabular}{|c|lll|}
\hline NEXT GENERATION NUCLEAR PLANT & Identifier: & PLN-2498 \\
METHODS RESEARCH AND & Revision: & 1 & \\
DEVELOPMENT TECHNICAL & Effective Date: & $9 / 25 / 08$ & Page: iv of iv \\
PROGRAM PLAN & & & \\
\hline
\end{tabular}

\section{EXECUTIVE SUMMARY}

One of the great challenges of designing and licensing the Very High Temperature Reactor (VHTR) is confirming that the intended VHTR analysis tools can be used confidently to make decisions, thereby ensuring that all reactor systems are safe and compliant with the performance objectives of the Generation IV Program. The research and development (R\&D) methods defined in this technical program plan will ensure that the tools used to perform the required calculations and analyses can be trusted. The R\&D tasks are designed to ensure that the calculational envelope of the tools used to analyze the VHTR reactor systems encompasses or is larger than the operational and transient envelope of the VHTR itself.

The R\&D methods presented in this plan focus on the development of tools to assess the neutronic and thermal fluid behavior of the Next Generation Nuclear Plant (NGNP). The fuel behavior and fission product transport models are discussed in the Advanced Gas Reactor Program Plan. Various stress analysis and mechanical design tools will also need to be developed and validated, which will ultimately be included in this technical program plan.

The calculational envelope of the neutronics and thermal-fluids software tools intended to be used on the NGNP is defined by the scenarios and phenomena that these tools can calculate with confidence. The software tools can only be used confidently when the results they produce have been shown to be in reasonable agreement ${ }^{\mathrm{a}}$ with first-principle results, thought-problems, and data that describe the highly ranked phenomena inherent in all operational conditions and important accident scenarios for the VHTR.

The $R \& D$ methods process is outlined in Figure E-1. The requirements associated with identifying the scenario, defining the Phenomena Identification and Ranking Tables (PIRTs), completing the required development, and performing the necessary validation studies must all be completed before the required analyses can be performed with confidence.

The present status of methods to be used in analyzing the VHTR is as follows:

- Software tools and methods needed to design and analyze the VHTR do no meet the Generation IV standards required for the NGNP. Considerable validation and probably, development of the necessary tools, are required.

- The software capabilities needed to perform

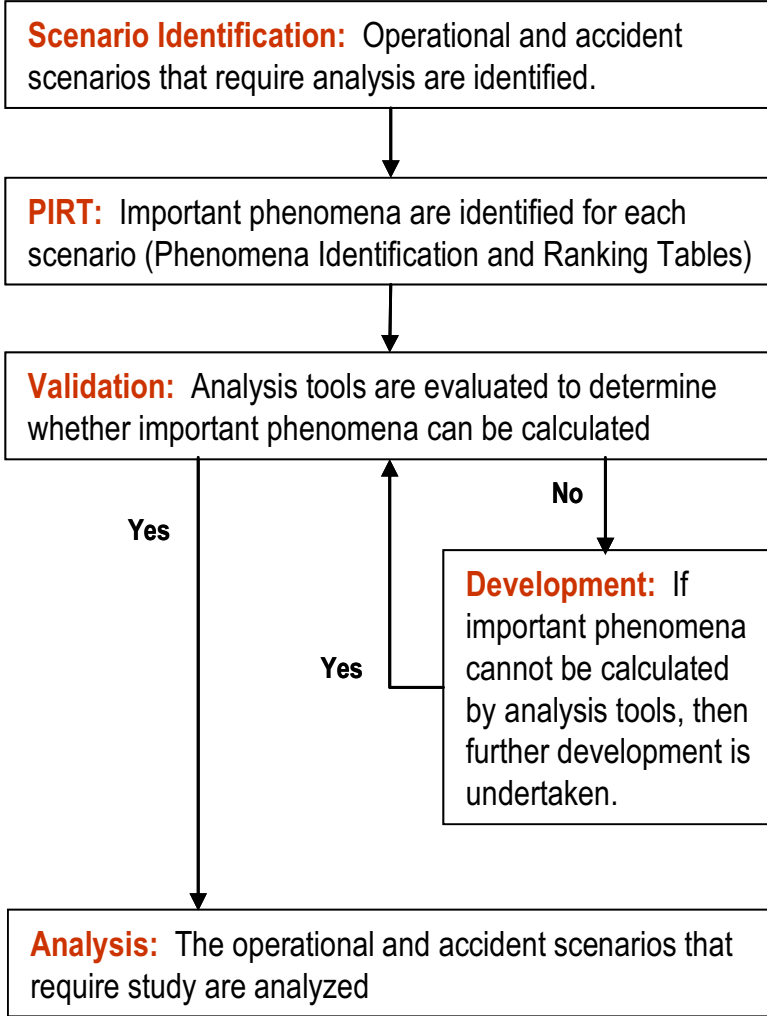

Figure E-1. PIRT informed R\&D process. NGNP licensing calculations to achieve Generation IV system objectives need to be developed and validated.

a. Reasonable agreement is achieved when the calculation generally lies within the uncertainty band of the data used for validation and always shows the same trends as the data. Code deficiencies are minor. 


\begin{tabular}{|c|lll|}
\hline NEXT GENERATION NUCLEAR PLANT & Identifier: & PLN-2498 \\
METHODS RESEARCH AND & Revision: & 1 & \\
DEVELOPMENT TECHNICAL & Effective Date: & $9 / 25 / 08$ & Page: $v$ of v \\
PROGRAM PLAN & & & \\
\hline
\end{tabular}

- The practices and procedures acceptable for both validating and developing the necessary software tools for the NGNP need to be defined and implemented to the satisfaction of the community.

These conclusions are true because (a) the key phenomena for the most challenging scenarios that must be analyzed for the to-be-selected VHTR have not yet been identified, (b) software tools that have a low calculational uncertainty will be required to analyze the behavior of the VHTR to enable the plant to operate at a high efficiency with a competitive economic margin, and (c) most of the software tools that will be used have not been validated for the scenarios and phenomena that must be analyzed. For instance, although systems analysis software has been validated for selected cases, a full validation has not been performed, nor are the data available that will enable a full validation to be performed. Also, the computational fluid dynamics software that will be widely used to analyze VHTR behavior has never been used in large measure to audit, design, or license calculations for a nuclear plant.

The VHTR design has not yet been selected. Consequently, the R\&D process is focused on scenarios and highly ranked phenomena that have already been identified as important by the advanced gas-cooled reactor community for the designs being considered as candidates for the VHTR. This approach has resulted in a VHTR-specific PIRT from which R\&D methods are being defined using the following assumptions:

1. The selected VHTR design could be either a pebble-bed or a block-type reactor.

2. The calculational and experimental needs, and consequently the required $\mathrm{R} \& \mathrm{D}$, are focused in the following eight distinct areas based on the relative state of the software in each:

a. Basic differential and integral nuclear cross-section data measurement and evaluation, including mathematically rigorous sensitivity studies of the effects of uncertainties in the differential nuclear data and other independent design variables on key integral reactor properties (the task of characterizing the effects of the nuclear fuel, fission products, moderator, and other relevant materials on the system reactivity, neutron flux distribution, and power production)

b. Reactor assembly cross-section preparation (the task of translating the fundamental data characterized in area (a) into formats and states useful for analysis)

c. Discrete ordinates transport (the process of approximating the neutron flux in a tractable manner for analysis)

d. Nodal diffusion (calculation of the energy and spatial flux profiles, reaction rates, reactivity changes, etc.)

e. Reactor kinetics (calculation of spatial changes in flux and power level as functions of time during postulated transients)

f. Thermal-fluids (the models that describe the fluid behavior and heat transfer behavior during steady-state and transient conditions for the scenarios of interest)

g. Fuel behavior

h. Fission product transport (determination of fission product movement once fission products have escaped from the confines of the fuel).

The methods R\&D is tailored to follow the guidance and timelines defined by the Energy Policy Act of 2005 (Public Law 109-58, 2005). That is, between now and perhaps until 2011, methods R\&D will be performed to enable analyses to be performed that can characterize the behavior of the candidate VHTR designs. The period beginning from the passage of the Energy Policy Act until the design is selected is Phase 1. Phase 2 will begin when Phase 1 is completed. During Phase 2 validation of the software tools 


\begin{tabular}{|c|lll|}
\hline NEXT GENERATION NUCLEAR PLANT & Identifier: & PLN-2498 \\
METHODS RESEARCH AND & Revision: & 1 & \\
DEVELOPMENT TECHNICAL & Effective Date: & $9 / 25 / 08$ & Page: vi of vi \\
PROGRAM PLAN & & & \\
\hline
\end{tabular}

will be completed using data directly scaled to the NGNP design and the operational, off-normal, and accident behavior of the design will be analyzed.

Commercial companies such as Areva, Ltd and PBMR Pty, which are currently designing future gascooled reactors, are still, in large measure, using legacy analysis tools to describe the operating and accident characteristics of their designs, and they intend to use them for licensing purposes. Recent visits of methods R\&D personnel in the NGNP Design and Safety Methods Validation Program to Areva, Ltd and PBMR Pty headquarters have fostered important interactions between commercial and national laboratory researchers. These interactions have shown that ongoing R\&D being performed at the national labs is on track to produce high-quality NGNP design and evaluation tools that are independent of the commercial companies and in keeping with Generation IV system objectives. These tools will not only be available to the Department of Energy (DOE) and the U.S. Nuclear Regulatory Commission (NRC), they may also be used by the vendors via agreements with DOE.

Between March and August 2006, NGNP methods R\&D personnel interacted numerous times with vendors and the thermal gas-cooled reactor community. In general, each of these meetings resulted in the same findings.

The NGNP Design Methods Workshop was held in Salt Lake City, Utah on August 22 and 23, 2006, to discuss the direction being taken in methods R\&D planning. The audience consisted of technical staff from the NRC, three national laboratories, two vendors, a consulting firm, and three universities. The reactor physics and thermal fluids areas were about equally represented by the attending experts from these organizations. The workshop was convened to review the research and development activities presently ongoing in methods $R \& D$ as well as the future program plans. The discussion centered on whether gaps exist between the program needs and the present R\&D tasks/plans. If gaps were identified, the workshop would then focus on how to eliminate them. Attendees determined that all of the identified gaps, issues, and inadequacies are currently being addressed, either directly, by formulating the practices and procedures to be used for planned verification and validation, or are planned to be implemented at some stage. Further details are presented in Appendix B of this plan.

In general, methods R\&D activities are divided into five distinct areas, as shown in Figure E-2: PIRT, nuclear data $R \& D$, neutronics methods validation $R \& D$, thermal-fluids methods validation $R \& D$, and analysis and evaluation. 


\begin{tabular}{|c|lll|}
\hline NEXT GENERATION NUCLEAR PLANT & Identifier: & PLN-2498 \\
METHODS RESEARCH AND & Revision: & 1 & \\
DEVELOPMENT TECHNICAL & Effective Date: & $9 / 25 / 08$ & Page: vii of vii \\
PROGRAM PLAN & & & \\
\hline
\end{tabular}

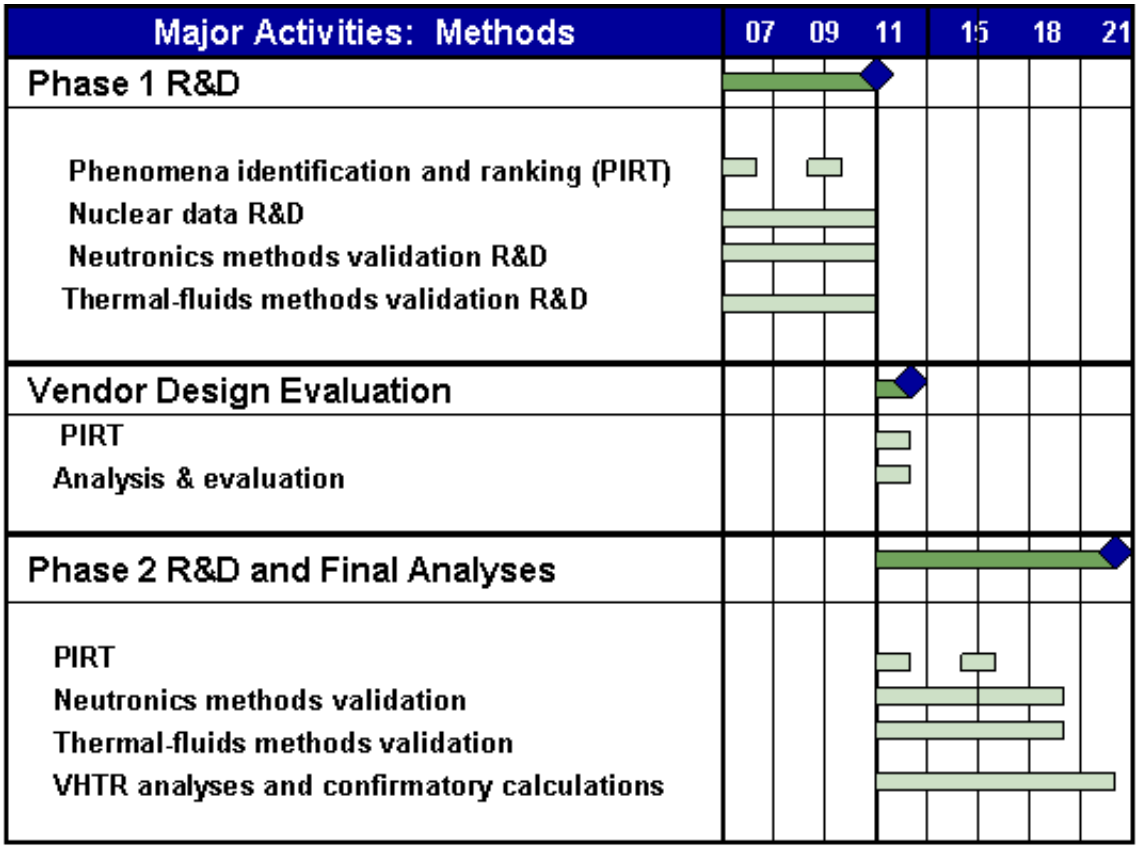

Task completion

Figure E-2. General activities associated with methods R\&D.

The highest-priority R\&D for Fiscal Years 2007, 2008, and 2009 is aimed at properly calculating the thermal-fluid conditions in the lower plenum during normal operation, developing and validating neutronics techniques that are necessary for analyzing both prismatic and pebble-bed reactors (PBRs), and analyzing the behavior of the plant during depressurized conduction cooldown and pressurized conduction cooldown accident scenarios.

Subsequent years will sharpen the focus in these and other areas that require analyses with low uncertainties for the most challenging scenarios identified by the PIRTs. The PIRTs form the heart of methods R\&D in that the R\&D needs are both identified and prioritized. Hence the PIRTs used to govern the R\&D needs will be updated throughout the cycle leading to the construction of the VHTR at INL. 


\begin{tabular}{|c|c|c|c|}
\hline $\begin{array}{c}\text { NEXT GENERATION NUCLEAR PLANT } \\
\text { METHODS RESEARCH AND } \\
\text { DEVELOPMENT TECHNICAL } \\
\text { PROGRAM PLAN }\end{array}$ & $\begin{array}{l}\text { Identifier: } \\
\text { Revision: } \\
\text { Effective Date: }\end{array}$ & $\begin{array}{l}\text { PLN-2498 } \\
1 \\
9 / 25 / 08\end{array}$ & Page: viii of vii \\
\hline
\end{tabular}

\section{CONTENTS}

EXECUTIVE SUMMARY iv

ACRONYMS xiv

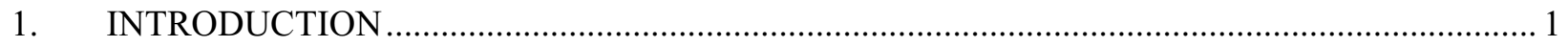

1.1 Role of NGNP Design and Safety Methods Validation Program ........................................ 1

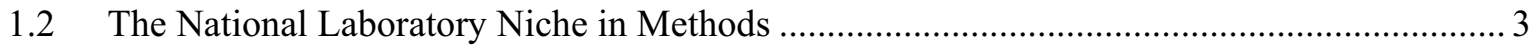

1.3 Qualification Process and Planning Approach.................................................................... 4

1.4 Qualification Procedures Based on NRC Regulatory Guide 1.203 Requirements ................ 4

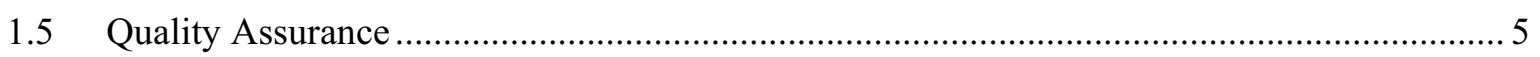

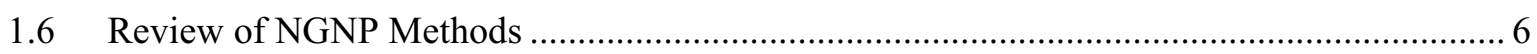

2. NGNP METHODS-THE KEY TO MAXIMIZING NGNP PLANT OPERATIONAL EFFICIENCIES, MINIMIZING UNCERTAINTIES, AND OPTIMIZING SAFETY

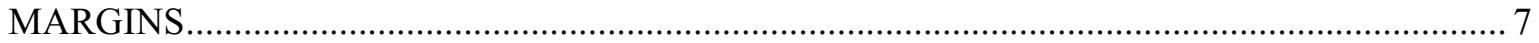

2.1 Conservative vs. Best-Estimate Analysis Methods............................................................. 7

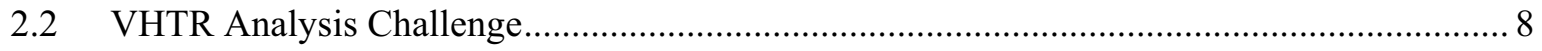

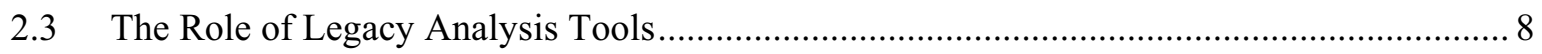

2.4 Mission of the Design and Safety Methods Validation Program .......................................... 9

2.5 Advantages of Advanced Analysis Tools ....................................................................... 9

2.6 Economics of Using Advanced Analysis Techniques....................................................... 9

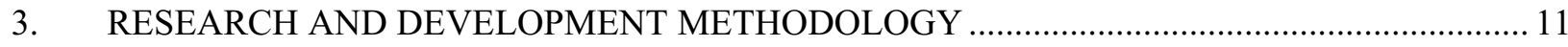

3.1 Scenario Identification and Phenomena Identification and Ranking Tables ....................... 12

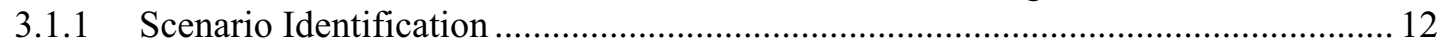

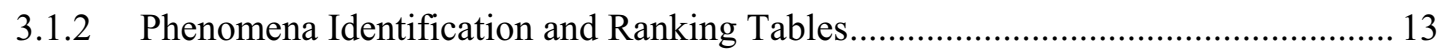

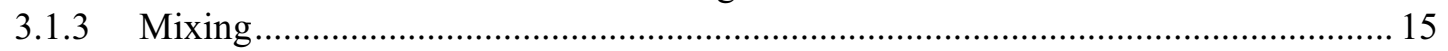

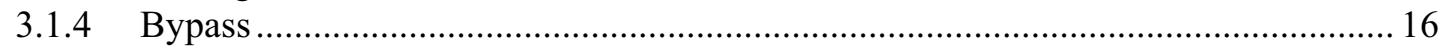

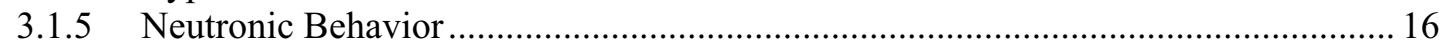

3.1.6 Laminar-Turbulent Transition Flow and Forced-Natural Mixed Convection

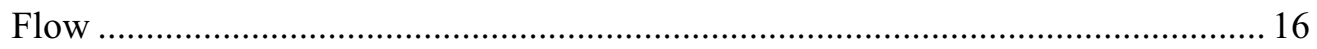

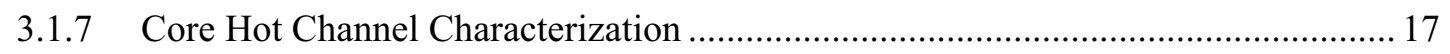

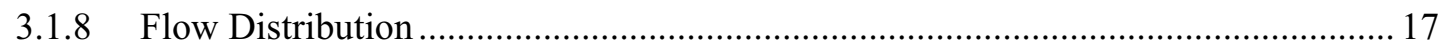

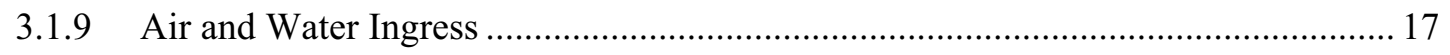

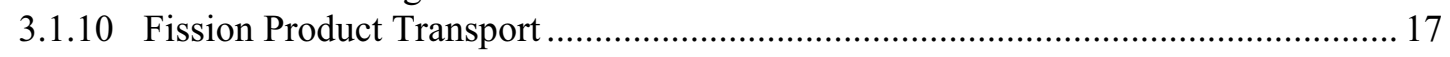

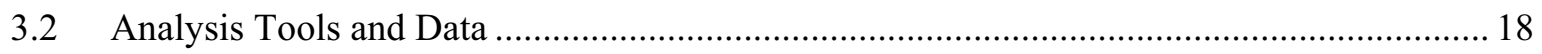

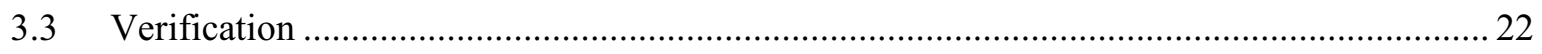

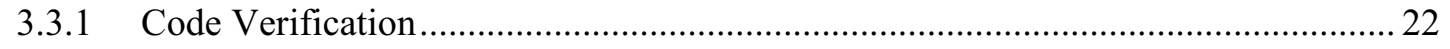

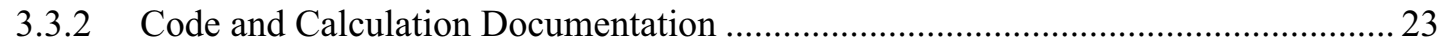

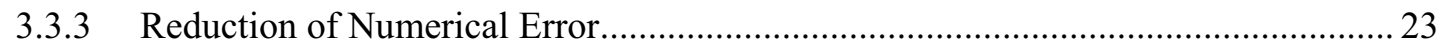

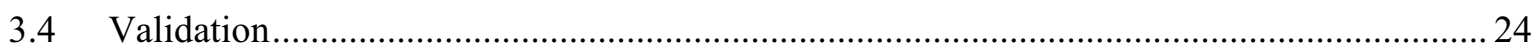

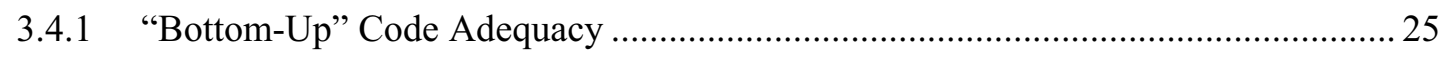




\begin{tabular}{|c|lll|}
\hline NEXT GENERATION NUCLEAR PLANT & Identifier: & PLN-2498 \\
METHODS RESEARCH AND & Revision: & 1 & \\
DEVELOPMENT TECHNICAL & Effective Date: & $9 / 25 / 08$ & Page: ix of ix \\
PROGRAM PLAN & & & \\
\hline
\end{tabular}

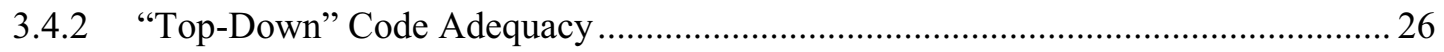

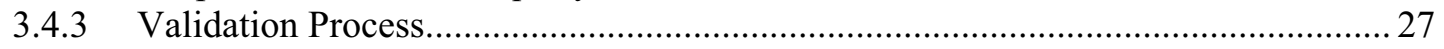

3.5 Software Tool Selection and Software Development ….................................................... 28

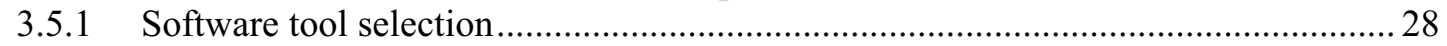

3.6 Analysis of Operational and Accident Scenarios and Expert Review .................................29

3.6.1 Summary of Meetings with PBMR Pty and Areva Ltd ........................................... 30

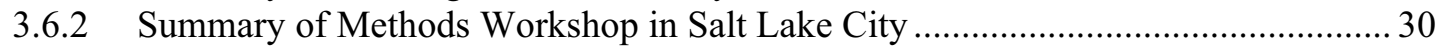

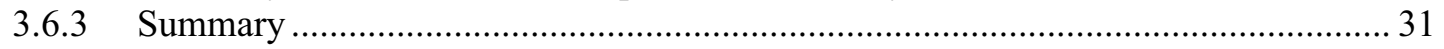

4. NUCLEAR DATA: INTEGRAL EXPERIMENT EVALUATIONS, SENSITIVITY

STUDIES, AND DIFFERENTIAL CROSS SECTION MEASUREMENTS .............................. 32

4.1 Integral Neutronic Parameter Evaluations for V\&V and Assessment of Needs for

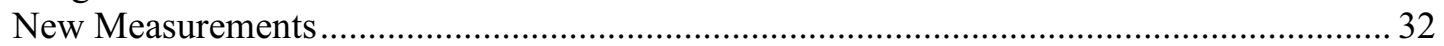

4.1.1 Integral Experiment Assessment for VHTR Methods Validation

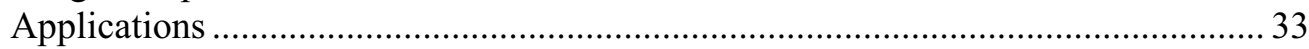

4.1.2 Protocols for Evaluating Integral Nuclear Data for Quality Assurance

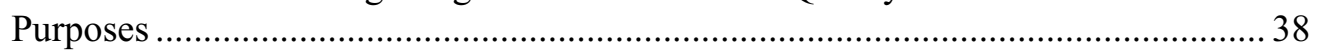

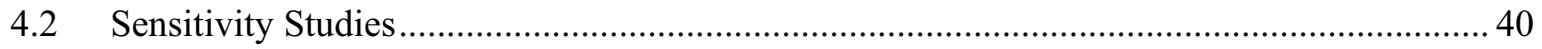

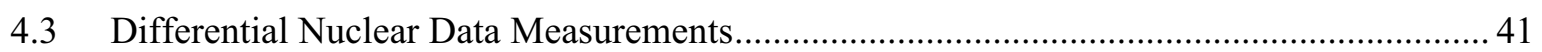

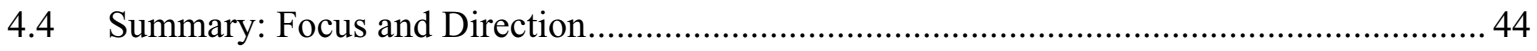

5. NEUTRONICS AND REACTOR KINETICS ANALYSIS DEVELOPMENT …........................ 46

5.1 Unit Cell and Assembly Analysis for Cross Section Generation......................................... 53

5.1.1 Method for Improved Treatment of Double Heterogeneity Using Improved

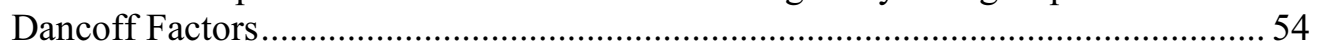

5.1.2 COMBINE Spectrum Code for Few-Group Cross Section Generation...................... 55

5.1.3 Argonne National Laboratory Assembly Code for Prismatic Block Crosssection Generation................................................................................................ 57

5.2 Static Analysis for Evaluations of Criticality and Power Distribution (Core

Simulation)

5.2.1 General Considerations in Static Core Simulation with Diffusion and Transport Theory.

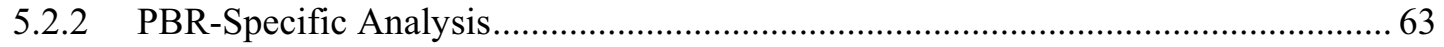

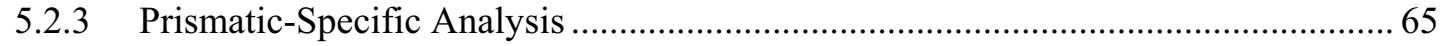

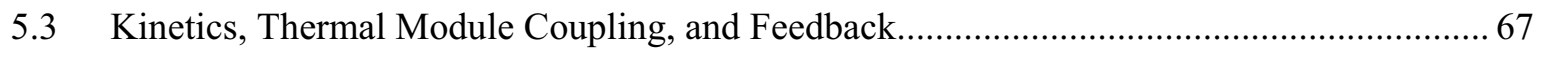

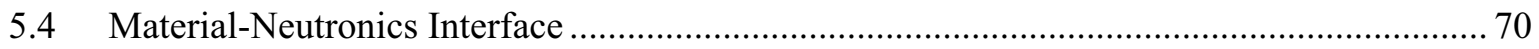

5.5 Improvement, Validation, and Verification (V\&V) of Code Suite.................................... 72

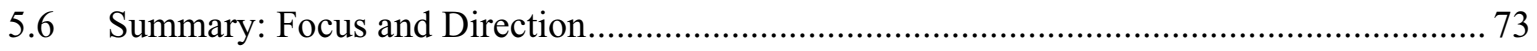

6. THERMAL-FLUID BEHAVIOR AND STRUCTURE TEMPERATURES …........................... 75

6.1 Introduction to CFD and Systems Analysis Code Validation Experiments.......................... 78

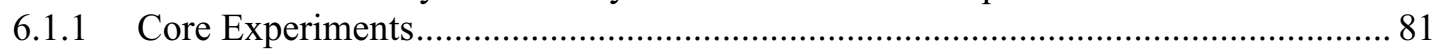

6.1.2 Upper and Lower Plenum Fluid Behavior Experiments ..................................... 86

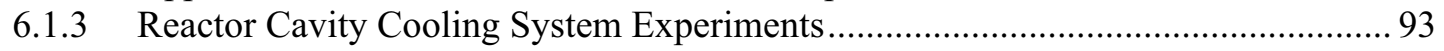

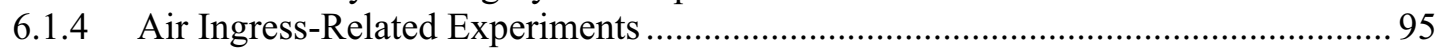




\begin{tabular}{|c|lll|}
\hline NEXT GENERATION NUCLEAR PLANT & Identifier: & PLN-2498 \\
METHODS RESEARCH AND & Revision: & 1 & \\
DEVELOPMENT TECHNICAL & Effective Date: & $9 / 25 / 08$ & Page: $\mathrm{x}$ of $\mathbf{x}$ \\
PROGRAM PLAN & & & \\
\hline
\end{tabular}

6.1.5 Larger-Scale Vessel Experiments and Prototypical Concept Experiments............... 97

6.2 Introduction to Thermal-Fluids Design Methods Development, Validation, and

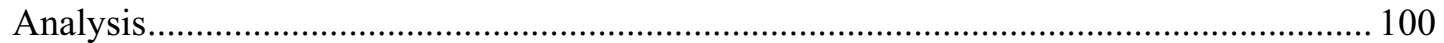

6.2.1 Methodology for Determining the Computational Fluid Dynamics Software........ 101

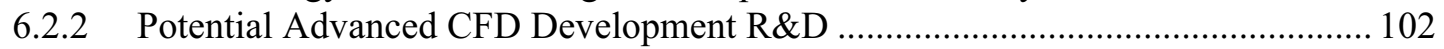

6.2.3 Core Heat Transfer Model Validation, Development, and Analyses....................... 103

6.2.4 Upper and Lower Plenum Flow Mixing Validations, Development, and

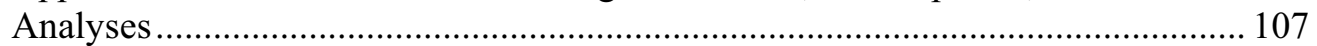

6.2.5 Reactor Cavity Cooling System Validations, Development, and Analyses............ 108

6.2.6 Air-Ingress Validations, Development, and Analyses ............................................ 112

6.2.7 Integral System Behavior Validations, Development, and Analyses...................... 113

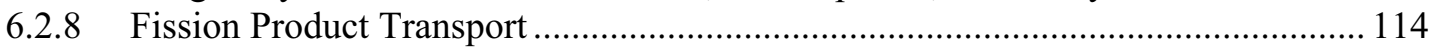

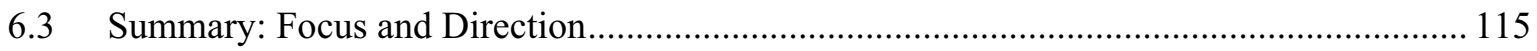

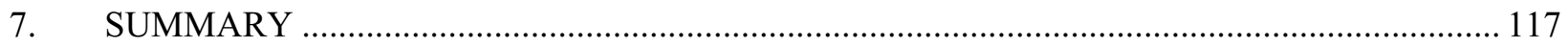

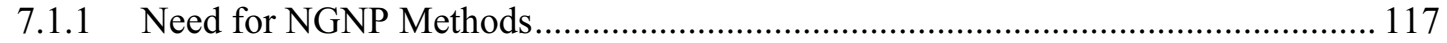

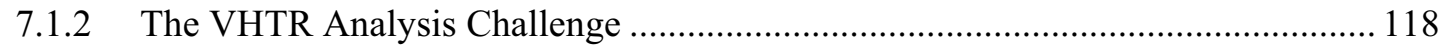

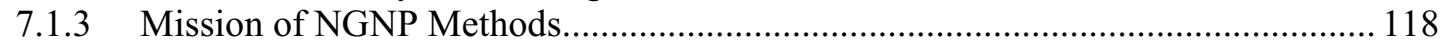

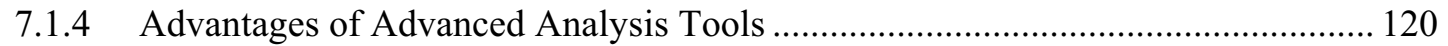

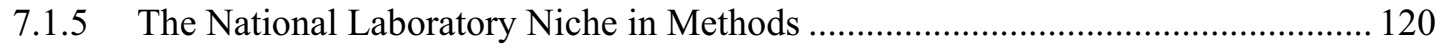

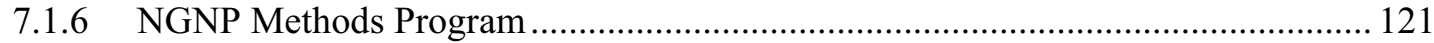

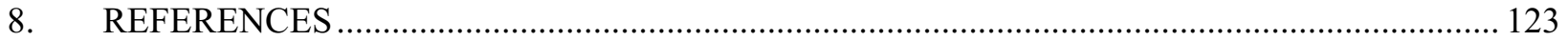

Appendix A—System Description and Phenomena Identification and Ranking Tables....................... 129

Appendix B-Summary of Next Generation Nuclear Plant Methods Workshop, Salt Lake City,

UT August 22-23, 2006

Appendix C - Differential Nuclear Data Measurements at the ANL Intense Pulsed Neutron

Source

Appendix D—The Use of Nuclear Energy for Process Heat.

\section{FIGURES}

Figure E-1. PIRT informed R\&D process. iv

Figure E-2. General activities associated with methods R\&D.................................................................vii

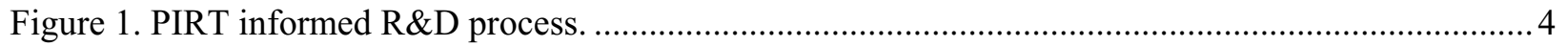

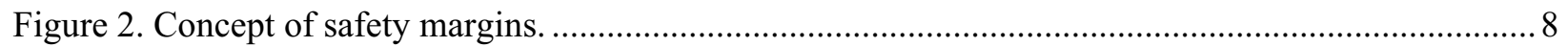

Figure 3. Expanded description of methods R\&D process.................................................................... 11

Figure 4. Computational fluid dynamics calculation of mixing in lower plenum (Courtesy of Fluent Corp)

Figure 5. Reactor Cavity Cooling System Configuration 


\begin{tabular}{|c|lll|}
\hline NEXT GENERATION NUCLEAR PLANT & Identifier: & PLN-2498 \\
METHODS RESEARCH AND & Revision: & 1 & \\
DEVELOPMENT TECHNICAL & Effective Date: & $9 / 25 / 08$ & Page: xi of xi \\
PROGRAM PLAN & & & \\
\hline
\end{tabular}

Figure 6. Convection flow regimes at various operating pressures for both helium and $\mathrm{CO}_{2}$ (from Williams, et al, 2003)

Figure 7. Calculation process.

Figure 8a. Application of process to pebble-bed candidate designs for NGNP with applicable software.

Figure $8 \mathrm{~b}$. Application of process to prismatic candidate designs for NGNP with applicable software.

Figure 9. VHTR system design software - elements of adequacy evaluation and acceptance testing practices.

Figure 10. Validation process, including participation by experts from the national laboratories, universities, vendors, and the community specific to the software undergoing validation.

Figure 11. Flow chart to evaluate applicability of analysis software.

Figure 12. Fission (left) and capture (right) cross sections for ${ }^{240} \mathrm{Pu}$. ENDF/B. Version 6 evaluations are shown with key original data superimposed.

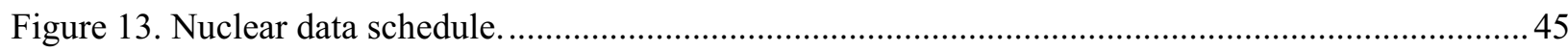

Figure 14. Typical HTGR spectrum and some low-lying resonances. ................................................5

Figure 15. Thermal neutron spectrum computed by COMBINE (version 6.02) and MCNP for a

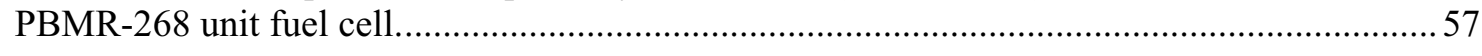

Figure 16. Comparison of VHTR spectra generated by various codes (ANL) ....................................58

Figure 17. Thermal neutron flux profile in the NGNP $600 \mathrm{MWt}$ reactor calculated by PEBBED.

Figure 18. Pebble flow lines (left) and sketch of the defueling cones near the bottom of the PBMR core (right).

Figure 19. Temperature trajectory during a DLOFC event in the PBMR ….......................................... 68

Figure 20. Ab initio (first principles) modeling of imperfections in a graphite crystalline structure.......... 71

Figure 21. Time line for neutronic and reactor kinetics analysis development. ...................................... 74

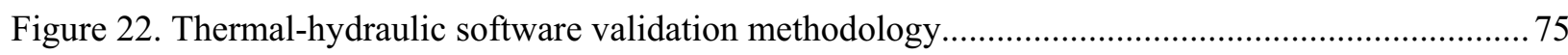

Figure 23. Core high power zones generate very hot exit gas and lead to high temperatures at turbine inlet or other power conversion equipment (IHX).

Figure 24. Preliminary assessment of popular turbulence models for flow in a vertical circular tube such as a prismatic NGNP cooling channel.

Figure 25. Potential apparatus to obtain benchmark turbulence data in heated channel flow. .82

Figure 26. Schematic diagram of miniature five-sensor probe by Vukoslavcevic and Wallace (2003), the dimensions are in millimeters.

Figure 27. Examples of some possible flow paths in the lower plenum of a typical VHTR block reactor concept. 


\begin{tabular}{|c|lll|}
\hline NEXT GENERATION NUCLEAR PLANT & Identifier: & PLN-2498 \\
METHODS RESEARCH AND & Revision: & 1 & \\
DEVELOPMENT TECHNICAL & Effective Date: & $9 / 25 / 08$ & Page: xii of xii \\
PROGRAM PLAN & & & \\
\hline
\end{tabular}

Figure 28. Matched-Index-of-Refraction flow system and a conceptual model design to study important flow features in a VHTR lower plenum.

Figure 29. Conceptual design of model for the anticipated first MIR lower plenum experiment (plan view).

Figure 30. Schematic diagram of benchmark experiment to simulate effect of buoyancy on thermal mixing in a lower plenum. 91

Figure 31. Schematic of ANL Natural Convection Shutdown Heat Removal Test Facility......................92

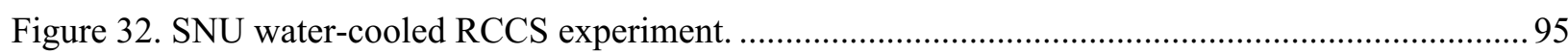

Figure 33. Schematic of HTR-10 (from HTGR Proceedings, Beijing, China, March 2001)....................98

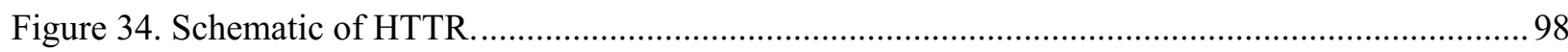

Figure 35. Approach for achieving validation objectives for CFD .................................................... 101

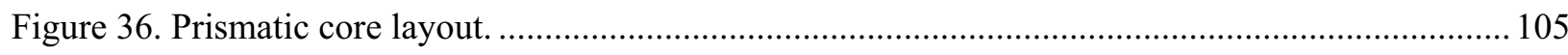

Figure 37. Prismatic block reactor core primitive............................................................................. 105

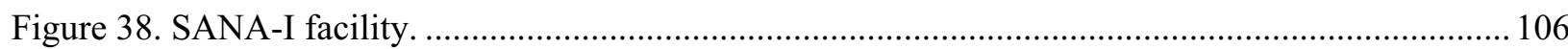

Figure 39. Preliminary calculation of mixing in prismatic reactor lower plenum. Courtesy of Fluent, Inc and General Atomics Corp. 107

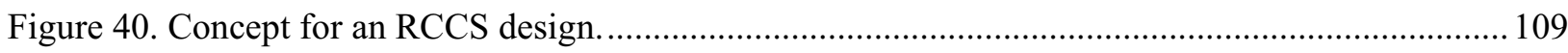

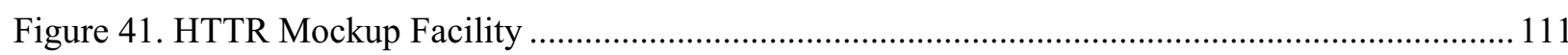

Figure 42. Timeline for thermal-fluid and structures thermal analysis............................................. 115

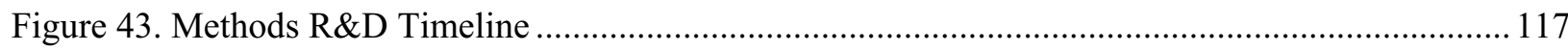

\section{TABLES}

Table 1. PIRT results for normal operation, PCC, and DCC scenarios............................................... 14

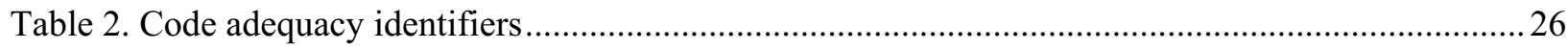

Table 3. Comparison of facilities for Pebble-Bed type cores for benchmarking models. ........................ 34

Table 4. Comparison of Facilities for Prismatic Block-Type cores for Benchmarking Models.................35

Table 5. Spectral indices as computed by MCNP and MICROX-2 ....................................................55

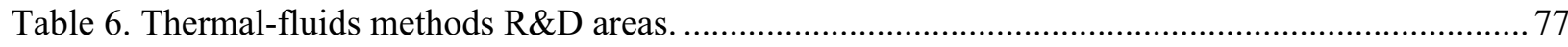

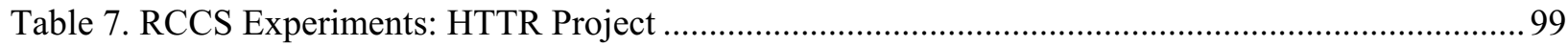




\begin{tabular}{|c|lll|}
\hline NEXT GENERATION NUCLEAR PLANT & Identifier: & PLN-2498 \\
METHODS RESEARCH AND & Revision: & 1 & \\
DEVELOPMENT TECHNICAL & Effective Date: & $9 / 25 / 08$ & Page: xiii of xiii \\
PROGRAM PLAN & & & \\
\hline
\end{tabular}




\begin{tabular}{|c|lll|}
\hline NEXT GENERATION NUCLEAR PLANT & Identifier: & PLN-2498 \\
METHODS RESEARCH AND & Revision: & 1 & \\
DEVELOPMENT TECHNICAL & Effective Date: & $9 / 25 / 08$ & Page: xiv of xiv \\
PROGRAM PLAN & & & \\
\hline
\end{tabular}

\section{ACRONYMS}

AVR Arbeitsgemeinschaft Versuchsreaktor

ANL Argonne National Laboratory

CFD computational fluid dynamics

CFR Code of Federal Regulations

DCC depressurized conduction cooldown scenario

DOE U.S. Department of Energy

FSAR Final Safety Analysis Report

FY fiscal year

GIF Generation IV International Forum

GTMHR gas turbine modular helium reactor

HTGR high temperature gas reactor

HTR-10 High Temperature Gas-Cooled Reactor (Chinese)

HTR high-temperature reactor

HTTR High-Temperature Test Reactor

IAEA International Atomic Energy Agency

IHX intermediate heat exchanger

I-NERI International Nuclear Energy Research Initiative

INET Institute of Nuclear Energy Technology

INL Idaho National Laboratory

IPNS intense pulsed neutron source

JAEA Japan Atomic Energy Agency (formerly JAERI)

LWR light-water reactor

LANSCE Los Alamos Neutron Science Center

MCNP Monte Carlo N-Particle (code)

MWe megawatts-electrical

MWt megawatts-thermal

NCSU North Carolina State University

NGNP Next Generation Nuclear Plant

NRC U.S. Nuclear Regulatory Commission

PBMR pebble-bed modular reactor

PBR pebble-bed reactor 


\begin{tabular}{|c|lll|}
\hline NEXT GENERATION NUCLEAR PLANT & Identifier: & PLN-2498 & \\
METHODS RESEARCH AND & Revision: & 1 & \\
DEVELOPMENT TECHNICAL & Effective Date: & $9 / 25 / 08$ & Page: xv of $\mathbf{x v}$ \\
PROGRAM PLAN & & & \\
\hline
\end{tabular}

PIRT phenomena identification and ranking tables

PMR prismatic modular reactor

PCC pressurized conduction cooldown scenario

RCCS reactor cavity cooling system

R\&D research and development

TRISO tri-isotopic, ceramic-coated-particle fuel

VHTR Very High Temperature Reactor

VSOP Very Superior Old Programs (a code system for reactor physics and fuel cycle analysis in pebble-bed reactors)

$\mathrm{V} \& \mathrm{~V} \quad$ verification and validation 


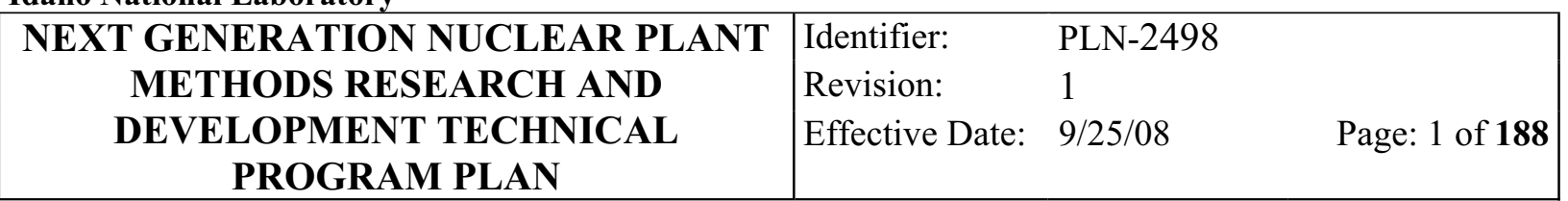

\section{INTRODUCTION}

President George W. Bush signed the Energy Policy Act of 2005 on August 8, 2005 (Public Law 109-58 2005). As summarized on page 15 of the September 2005 issue of Nuclear News, in the article "Compromise Energy Bill Becomes Law," the nuclear provisions specific to the Next Generation Nuclear Plant (NGNP) are as follows:

The DOE shall establish the Next Generation Nuclear Plant project, with a prototype to be sited at Idaho National Laboratory. The centerpiece is to be the development of reactor, fuel, and associated technology for the production of hydrogen as well as electricity. The DOE and the NRC are to submit jointly a licensing strategy to Congress within three years after enactment. Hydrogen production technology and initial reactor design parameters are to be chosen by September 30, 2011, or an alternative date is to be submitted to Congress by that time. The reactor is to begin operation by September 30, 2021, or an alternative date is to be submitted to Congress by that time. The project is authorized to receive $\$ 1.25$ billion over fiscal years 2006 through 2015, and such sums as are necessary thereafter.

Research and development (R\&D) specific to NGNP mentioned in the Energy Policy Act (2005) and conducted to date is based on the gas-cooled Very High Temperature Reactor (VHTR) concept promulgated in the Generation IV technology roadmap (Generation IV International Forum 2002). Presently, the most likely VHTR candidates are the prismatic and pebble-bed designs. ${ }^{b}$ Consequently, the $\mathrm{R} \& \mathrm{D}$ described in this document is focused on these types of gas-cooled thermal reactors. The fuel behavior and source term models are discussed in the Technical Program Plan for the Advanced Gas Reactor Fuel Development and Qualification Program (Petti, Hobbins, Kendall, and Saurwein 2005).

\subsection{Role of NGNP Design and Safety Methods Validation Program}

An important ingredient in the R\&D spectrum that must be accomplished to make the NGNP a reality is the development and validation of design and evaluation tools. Various design and analysis tools ${ }^{\mathrm{c}}$ are needed to calculate the behavior of the NGNP within its operating envelope and within its off-normal and accident envelopes. Thus, examples of products that will be provided by the NGNP Design and Safety Methods Validation Program are software tools and the pertinent experiments for their validation, or the benchmarking of these tools that will allow the plant operational and licensing requirements to be calculated:

1. Specific analysis tools are needed for off-normal and accident conditions to calculate:

a. The prescribed distribution of the fuel in the core both initially and at any time during the life of the reactor. The fuel in the prismatic reactors is fixed, but the capability to define where each fuel element should be located (given a defined enrichment), both initially and during reloads, and the capability to calculate the burnup histories of each fuel element over its tenure in its prescribed location are essential. The fuel in the pebble-bed reactor (PBR) begins its journey through the

b. The Fort St. Vrain power plant was a prismatic configuration (also called a block-type) reactor, and the German Arbeitsgemeinschaft Versuchsreaktor (AVR) was a pebble-bed configuration reactor.

c. End products are usually computer software, analysis reports, comparison with experimental results (physical benchmarks) and procedures for performing these analyses. 
Identifier: $\quad$ PLN-2498

Revision: $\quad 1$

Effective Date: $9 / 25 / 08$

core as a function of where it is initially placed at the top of the core; the trajectory of the pebbles and how they influence the core power at each point along their paths must be calculated.

b. The three-dimensional (3-D) core power distribution.

c. The helium coolant core flow distribution, including the bypass flows.

d. The flow distribution and flow dynamics throughout the remainder of the reactor vessel, for example in the outlet plenum.

e. The potential for cyclic flows and/or equipment flutter and vibration.

f. How to prescribe fuel enrichments and locations for reloads.

g. The graphite dust generation rate.

h. The graphite dust distribution throughout the system.

i. Whether localized hot spots exist throughout the system that may lead to premature structural failures.

j. Whether excessive thermal gradients are present that may lead to premature structural failures.

$\mathrm{k}$. The reactor vessel wall temperature distribution and the interaction between the reactor and the reactor cavity.

1. The parasitic energy losses to the reactor cavity and the cavity cooling system.

m. The inlet, in-component, and exit conditions (most likely two-dimensional [2-D] or 3-D) for the plant power conversion systems, for example an intermediate heat exchanger.

3. Specific analysis tools are needed for off-normal and accident conditions to calculate:

a. The leakage rates for various break configurations and the resulting effects on the system such as: graphite dust redistribution both within the reactor and through the leak to the reactor cavity, pressure pulse propagation, structural loads, depressurization rates, flow distributions within the reactor system resulting from prolonged leaks, and the effect of the leaks on the fluid dynamics and pressurization rates in the reactor vault (reactor cavity).

b. Potential reactivity events including the fuel power transients and reactivity interactions with the system.

c. The fuel temperatures and fuel failure rates.

d. The core temperature distribution throughout the various off-normal and accident scenarios.

e. Density-gradient flow distributions both for depressurized conduction cooldown and pressurized conduction cooldown scenarios including the presence of localized hot spots and potentially excessive thermal gradients.

f. The stratified flow behavior that will occur when a break unchokes and the flow from and into the vessel are governed by the density differences between the outgoing helium and incoming airfor densimetric Froude numbers less than one.

g. The air ingress into the core region, including the extent of graphite oxidation that will occur.

h. The fluid dynamics of potential water ingress scenarios.

i. The energy transfer between the reactor (and any discharge from the reactor) to the reactor cavity and the cavity cooling systems.

j. The potential for boiling and dryout of the cavity cooling working fluid (two of the candidate designs use water to cool the reactor cavity walls).

k. The trajectories of the released fission products and the fraction of the fission products that are released to the out-plant environment. 


\begin{tabular}{|c|lll|}
\hline NEXT GENERATION NUCLEAR PLANT & Identifier: & PLN-2498 \\
METHODS RESEARCH AND & Revision: & 1 & \\
DEVELOPMENT TECHNICAL & Effective Date: & $9 / 25 / 08$ & Page: 3 of 188 \\
PROGRAM PLAN & & & \\
\hline
\end{tabular}

1. The reactor system behavior during seismic events.

To demonstrate whether or not the NGNP analysis software is capable of analyzing the above phenomena and plant integral behavior, a set of data are required for almost every item identified for operational conditions, off-normal conditions, and accident conditions to allow a rigorous validation to be performed. In some cases the data are available, but need to be qualified. However, there are a considerable number of phenomena or plant integral behavior scenarios that require new experiments to be performed.

\subsection{The National Laboratory Niche in Methods}

The gas-cooled thermal reactors built and operated in the United States and elsewhere are, to date, characterized by sustained operations at conditions that provide or have substantial design and safety margins. The margins were designed to be large because the legacy analysis tools used to calculate limiting parameters on the systems were incapable of calculating important local limiting parameters with sufficient accuracy to reduce the safety margins to more desirable levels where the economics of the plant operational, off-normal, and accident envelopes could be optimized. Until recently, the analysis tools and the required boundary conditions (material properties and neutronics cross-sections) were unavailable, preventing the required parameters (such as localized power distributions and localized wall temperatures) from being calculated with a sufficiently low calculational uncertainty. This approach has resulted in sustained operating temperatures and efficiencies below the Generation IV system goals.

The commercial companies (for example Areva, Ltd, General Atomics, Inc and PBMR Pty) currently designing the future gas-cooled reactors are still, in large measure, using legacy analysis tools to describe the operating and accident characteristics of their designs, and they intend to use them for licensing purposes. Recent visits of Design and Safety Methods Validation Program personnel to the headquarters of Areva Ltd, General Atomics, Inc., and PBMR Pty have allowed commercial and national laboratory researchers to interact effectively (Schultz et al, 2006a, 2006b). The outcome of these interactions is that it is clear that the products of the Design and Safety Methods Validation Program will be of use to the vendors.

The Design and Safety Methods Validation Program will provide advanced, state-of-the-art tools that are independent of those used by the vendors. Most of these tools, which include a mixture of commercial software and software written in the national laboratories, are already available. Only limited tool development is needed. The major cost remaining focus on software verification and validation (V\&V). The cost distribution is projected to be approximately $\$ 120 \mathrm{M}$, with $16 \%$ for neutronics and $84 \%$ for thermal-fluids. ${ }^{\mathrm{d}}$ Although $\$ 120 \mathrm{M}$ is the projected amount required to complete the entire scope of work outlined by the Design and Safety Methods Validation Program, including software development, benchmark activities, and actual physical experiments, the cost will be more than is recovered by the capability of the plant to operate at peak thermal efficiencies with maximum outlet temperatures. A summary of the benefits stemming from achieving the objectives of the Design and Safety Methods Validation Program is given in Chapter 2 together with a brief economic analysis.

d. The cost distribution projections are heavily weighted towards the cost of required experiments. Once V\&V of the NGNP software is completed, the tools may be used by the vendors and will be available for use by DOE and the NRC. 


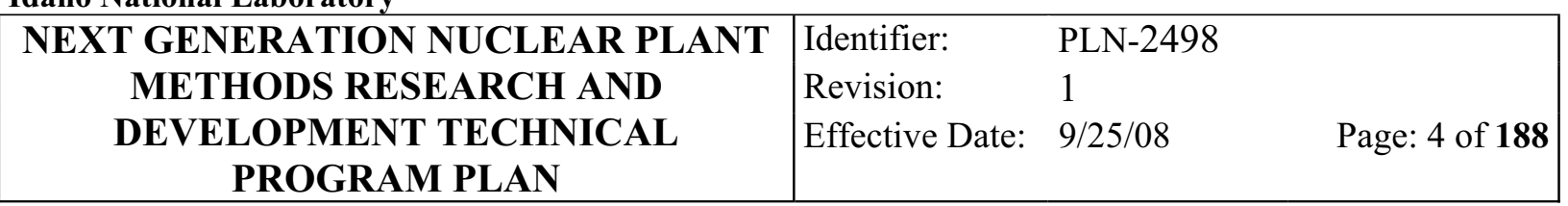

\subsection{Qualification Process and Planning Approach}

The process of identifying R\&D needs and then formulating plans is straightforward, even though there are many unknowns and the process itself is iterative. The process, shown in flowchart form in Figure 1, is a five stage process that consists of (1) identifying the scenarios of importance, (2) identifying the key phenomena for the scenarios of importance, (3) determining whether the tools to be used to analyze the scenario progressions are adequate, (4) correcting or completing existing software and carrying out any software development that may be needed to ensure that the analysis tools are adequate, and finally, (5) performing the required analyses.

Presently, the status of the methods to be used for analyzing the VHTR is:

- State-of-the-art software and advanced detailed methods are not ready to perform design and analysis to VHTR standards; the software tools require limited development and considerable validation.

- The above conclusion also applies to present software capabilities to perform VHTR licensing calculations.

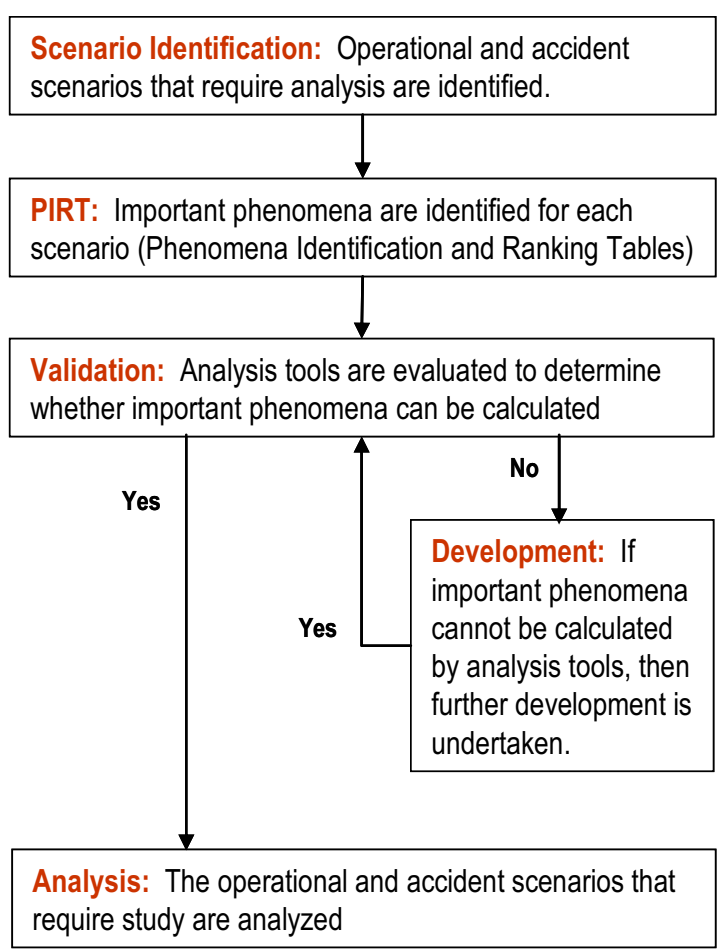

Figure 1. PIRT informed R\&D process.

- The practices and procedures acceptable for both validating and developing the necessary software tools for the VHTR must be defined and implemented to a standard defined by the community.

These conclusions are true because: (a) the key phenomena for the most challenging scenarios that must be analyzed for the to-be-selected VHTR have not been identified yet, (b) software tools that have a low calculational uncertainty will be required to analyze the behavior of the VHTR to enable the plant to operate at a high efficiency with a competitive economic margin, and (c) most of the software tools that will be used have not been validated for the scenarios and phenomena that must be analyzed for licensing. For example, even though systems analysis software has been validated for selected cases, a full validation has not been performed nor is the data available that will enable a full validation to be performed. Also, computational fluid dynamics (CFD) software, which will be widely used to analyze VHTR behavior, have never been used in large measure to perform auditing, design, or licensing calculations for a nuclear plant.

\subsection{Qualification Procedures Based on NRC Regulatory Guide 1.203 Requirements}

The U.S. Nuclear Regulatory Commission (NRC) describes a process in their Regulatory Guide 1.203 which they consider acceptable for use in developing and assessing evaluation models that may be used to analyze transient and accident behavior that is within the design basis of a nuclear power plant. In general, the Evaluation Model Development and Assessment Process (EMDAP) described in Guide 1.203 


\begin{tabular}{|c|c|c|c|}
\hline $\begin{array}{c}\text { NEXT GENERATION NUCLEAR PLANT } \\
\text { METHODS RESEARCH AND } \\
\text { DEVELOPMENT TECHNICAL } \\
\text { PROGRAM PLAN }\end{array}$ & $\begin{array}{l}\text { Identifier: } \\
\text { Revision: } \\
\text { Effective Date: }\end{array}$ & $\begin{array}{l}\text { PLN-2498 } \\
1 \\
9 / 25 / 08\end{array}$ & Page: 5 of $\mathbf{1 8 8}$ \\
\hline
\end{tabular}

consists of (1) determining the requirements for the evaluation model, ${ }^{\mathrm{e}}(2)$ developing an assessment base $\mathrm{f}^{\mathrm{f}}$ consistent with the determined requirements, (3) developing the evaluation model, (4) assessing the adequacy of the evaluation model, (5) following an appropriate quality assurance protocol during the EMDAP, and (6) providing comprehensive, accurate, up-to-date information.

Although a specific NGNP design has not been selected, the methods R\&D effort has proceeded by examining and postulating the evaluation model requirements in conjunction with making a preliminary formulation of the required assessment base (Lee, Wei, and Schultz et al 2005) in Steps 1 and 2. Because the NGNP will likely be either a prismatic or a pebble-bed type gas-cooled thermal reactor with known general characteristics, the various steady-state and transient characteristics are generally known. The assessment base (benchmark experiments) cannot be defined and selected until the final design selection, since many of the thermal-fluids experiments are very geometry specific and very dependent on initial conditions that would reflect initial operating and accident conditions. The most probable VHTR design basis scenarios are described in Chapter 3.

The evaluation models have been selected (Step 3), as will be noted in Chapter 3. A different suite of methods software is required to calculate the reactor physics behavior for the prismatic as opposed to the pebble-bed gas-cooled reactor. However, the software used to calculate the thermal-fluids behavior is the same for both reactor types.

In essence, much of this plan deals with defining (a) the appropriate experiments to enable methods software validation to meet the Regulatory Guide 1.203 requirements and (b) the practices and procedures that must be developed and used to ensure the evaluation models are deemed adequate. Thus, much of this plan addresses Steps 2 and 4 of the EMDAP; Chapters 4, 5, and 6 discuss these topics.

In summary, the methods $R \& D$ tasks are being planned and executed in compliance with the approach, practices, and methodologies recommended in Regulatory Guide 1.203.

\subsection{Quality Assurance}

Although the approach, methodologies, practices, and procedures used to define and design the Design and Safety Methods Validation Program follow Regulatory Guide 1.203, the Quality Assurance standards, practices, and procedures that govern the Design and Safety Methods Validation Program are those specified for INL and the U.S. Department of Energy (DOE), specifically, American Society of Mechanical Engineers (ASME) Standard NQA-1.

Specific actions needed for the Design and Safety Methods Validation Program to meet NQA-1 requirements are proceduralized in PLN-2889, "Requirements for NGNP Methods Software Quality Control" and in PLN-2888, "Requirements for Code Validation Experiments Performed in Support of NGNP Methods." These plans will be used to direct software validation activities performed at INL. When validation activities are performed at other sites, such as other national labs or universities, those sites will have the option of adopting the INL plans or creating their own corresponding procedures. Should other procedures be developed, the Design and Safety Methods Validation Program manager or designated alternate must approve the new procedure prior to use to ensure whatever procedures are used conform to the quality assurance requirements of the NGNP Project.

INL PLN-2889 for software quality fulfills Regulatory Guide 1.203 requirements, but also relies heavily on NQA-1 requirements. It applies to software modified, developed, or acquired by the Design

e. An evaluation model is the calculational framework for evaluating the behavior of the reactor system during a postulated transient or design-basis accident.

f. Either certifying existing experimental data as being adequate or designing physical experiments that will provide highquality, acceptable data. 


\begin{tabular}{|c|lll|}
\hline NEXT GENERATION NUCLEAR PLANT & Identifier: & PLN-2498 & \\
METHODS RESEARCH AND & Revision: & 1 & \\
DEVELOPMENT TECHNICAL & Effective Date: & $9 / 25 / 08$ & Page: 6 of 188 \\
PROGRAM PLAN & & & \\
\hline
\end{tabular}

and Safety Methods Validation Program to support DOE Office of Environmental Management validation by federal agencies. It may be used to support audits and reviews performed by federal agencies, such as the NRC and DOE.

The Software Quality Plan includes sections on problem reporting and corrective action, configuration control, required reviews, software procurement and acquisition, and records management. It includes roles and responsibilities and training requirements. It then follows the software life-cycle and presents requirements by life-cycle phase. Lastly, it presents example forms for use in meeting the documentation requirements stated in the plan.

INL PLN-2888 for validation experiments fulfills the requirements set forth in the Regulatory Guide 1.203 and NQA-1. Similar to software activities performed by the Design and Safety Methods Validation Program, the results are not intended for use by the program for nuclear plant design or construction. However, due to the complexity and cost of experimentation, it must be done to the highest standards so that other entities who may use the resulting data can be sure of its pedigree.

The Experiment Plan includes sections on configuration control, reviews, training, and roles and responsibilities. It discusses calibration requirements, records management, and management of discrepancies. The plan details the requirements for and contents of an acceptable experiment plan.

\subsection{Review of NGNP Methods}

Two techniques have been used to review the approach and plans of NGNP methods R\&D over the past year. The first reviews were done by two commercial vendors independent of one another: Areva Ltd and PBMR Pty. Areva Ltd designs prismatic-type reactors and PBMR Pty designs pebble-bed type helium-cooled thermal reactors. The reviews of these commercial vendors were in the context of a dialogue between national laboratory staff members who visited the vendor faculties with the intent to view toward determining areas of collaboration. A second review was done by a Methods Working Group in a workshop held specifically to determine what gaps, if any, existed in the NGNP methods planning. The outcome of the three reviews was the same: general agreement with the approach taken by the NGNP methods development effort and specific suggestions on (a) regions of collaboration between the national laboratories and the vendors and (b) how to improve the NGNP Design and Safety Methods Validation Program planning to date. The outcomes of these reviews are summarized in Chapter 3.

The remainder of this report is divided into seven sections: Chapter 2 summarizes the benefits and cost savings inherent to the achieving the goals of the Design and Safety Methods Validation Program; Chapter 3 defines the methodology for producing validated analytical tools for the analysis of the VHTR; Chapters 4, 5, and 6 detail the planned research program in the three key areas of nuclear data measurements, reactor kinetics and neutronics, and thermal-fluid behavior, respectively. Chapter 7 summarizes R\&D while Chapter 8 gives the references.

Following the main body of the report, five appendices are attached. Appendix A describes the phenomena identification and ranking table analyses for three of the scenarios that define the NGNP operating envelope together with a description of the gas-cooled VHTR design concepts including operating conditions and transients. Appendix B summarizes the results of the August 2006 NGNP Methods Workshop. Appendix C describes the differential nuclear data measurements that are needed using the Argonne National Laboratory (ANL) Intense Pulsed Neutron Source. Appendix D has a short discussion regarding the use of nuclear energy for process heat. Appendix E summarizes the cost and schedule for the Methods R\&D tasks. Appendix E is published separately and is available on request with the permission of the DOE. 


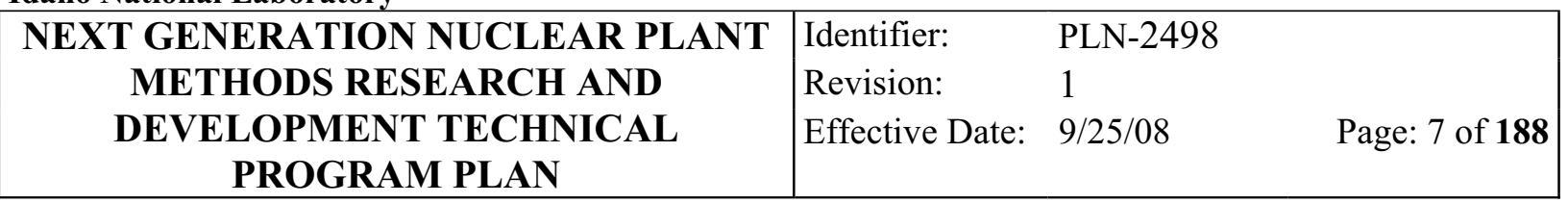

\section{NGNP METHODS-THE KEY TO MAXIMIZING NGNP PLANT OPERATIONAL EFFICIENCIES, MINIMIZING UNCERTAINTIES, AND OPTIMIZING SAFETY MARGINS}

Online nuclear power plants can only operate within limits defined by the capability of the licensee to demonstrate that all important figures-of-merit for plant safety parameters are never challenged-a sufficient margin must be observed so the figure-of-merits are not in danger of being violated. A figureof-merit is a key parameter indicative of whether or not a safety limit or an equipment failure limit has been breached. For example, important figures-of-merit are fuel temperature and reactor vessel wall temperature. Although there are a number of figures-of-merit crucial to the operation of a power plant, for the sake of demonstrating the importance of NGNP methods, the reactor vessel wall temperature will be used as an example for the following discussion. Equivalent arguments apply to all figures-of-merit.

Regardless of the figure-of-merit under consideration, each has the potential to limit the operational envelope of the plant in some way that restrictions such as special precautions, operational procedures, or equipment limitations will be required to ensure the figure-of-merit is not challenged. In some cases, the restrictions translate to operating the plant at power levels below the designed power level. In other cases, the restrictions may translate to reducing the rate-of-change of an operational parameter in going from one condition to another, thus increasing the operational transit time. In any case, such restrictions have an economic penalty.

\subsection{Conservative vs. Best-Estimate Analysis Methods}

Figures-of-merit for the present fleet of light-water reactors (LWRs) were traditionally calculated using conservative assumptions and approaches that were guaranteed to yield calculated results with very large safety margins. Models of this sort were based on a prescription of sometimes arbitrary restrictions (e.g., neglecting heat transfer for certain phases of a scenario) to ensure a large safety factor was present in the licensing calculations. The approach codified in Title 10 of Part 50.46, Appendix K of the Code of Federal Regulations is the most widely known example. The major drawback to the Appendix $\mathrm{K}$ approach is that the calculational uncertainty, while known to be large and conservative, is not quantified.

Subsequent to the Appendix K approach, best-estimate approaches were developed and have been used to perform some plant license reevaluations. The best estimate approaches have the advantage of enabling the calculational uncertainties to be defined and quantified. However, one-dimensional (1-D) fluid flow models were almost exclusively used to calculate average or bulk values of the figures-of-merit in the various regions of the plant. Thus, to account for potential deviations from the 1-D model results, because of 3-D behavior, safety factors have been used to provide a sufficient margin from the limiting value.

The concepts discussed in the above two paragraphs are illustrated in Figure 2. The best estimate approach for calculating the safety margin gives more operational latitude to the plant operator than using a conservative approach with prescribed arbitrary models. 


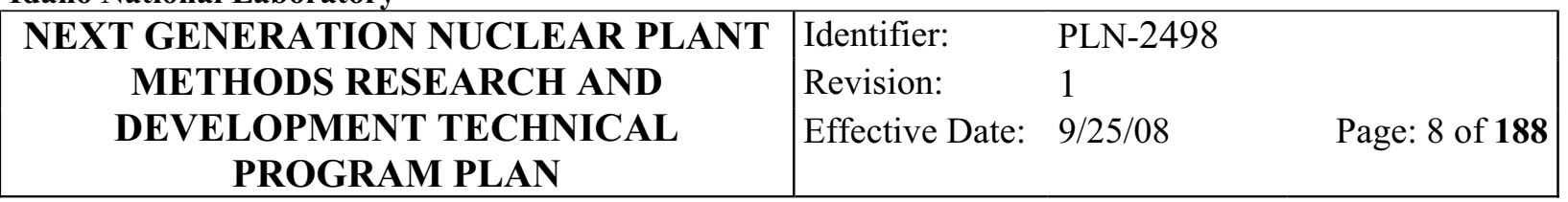

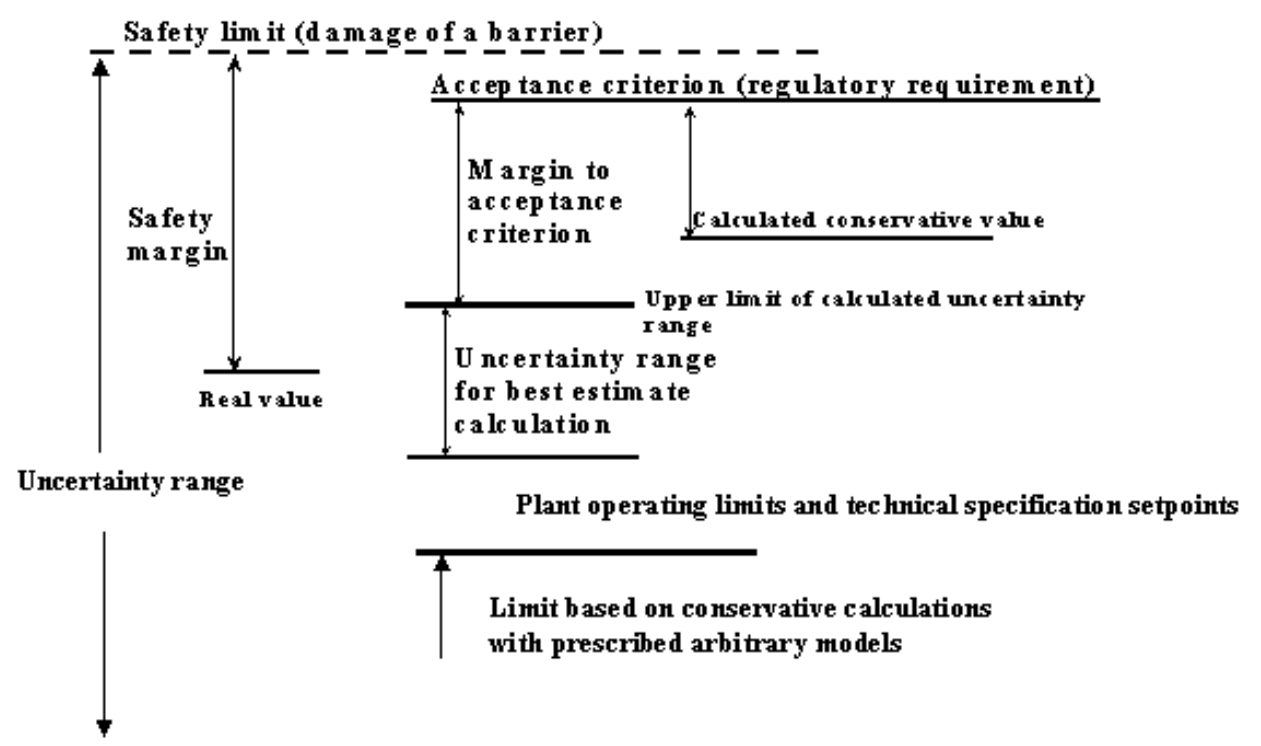

Figure 2. Concept of safety margins.

\subsection{VHTR Analysis Challenge}

1-D techniques were usually adequate for calculating the plant thermal-hydraulic behavior of LWRs because the fluid temperatures, even under the most arduous conditions, were considerably less than the temperatures that challenge the structural material limits, for example the reactor vessel. That is not the case for the VHTR.

Bulk outlet temperatures for the VHTR must be in excess of $900^{\circ} \mathrm{C}$ and as close to $1000^{\circ} \mathrm{C}$ as possible to (a) enable the system to operate at its maximum thermodynamic efficiency and (b) provide an optimal heat source to satisfy the NGNP mission requirements whether the mission is to generate process heat or hydrogen. Operation at bulk outlet temperatures in excess of $900^{\circ} \mathrm{C}$ and as close to $1000^{\circ} \mathrm{C}$ as possible is what is meant by having the VHTR meet Generation IV plant operating requirements. For bulk outlet temperatures of $950^{\circ} \mathrm{C}$, peak exit gas temperatures at particular outlet jet locations may be considerably above $1000^{\circ} \mathrm{C}$ at operational conditions, depending on the core power distribution and the core bypass. Subsequent mixing in the outlet plenum allows for the reduction of temperature in these plumes down to the bulk average temperature; however, the local gas jets exiting the core have the potential to impinge on the walls of the flow path and create localized hot spots if the design has not properly accounted for such behavior. This high temperature gas jet problem impacts materials placement and selection for the accident scenarios and operational plant considerations. Similar arguments apply to conditions that may exist for transient and the most challenging accident scenarios.

\subsection{The Role of Legacy Analysis Tools}

The vendors are using existing, legacy software to calculate the plant operational and accident behavior for licensing purposes. Legacy software performs a rigorous 1-D calculation of the bulk temperatures but does not have the capability to calculate localized hot spots. Hence prescribed safety factors are used to ensure that local material temperatures do not exceed material property limits. Since the prescribed safety factors account for the large uncertainties inherent in the use of legacy tools to calculate the localized core power distributions and maximum outlet jet temperatures, the VHTR will 


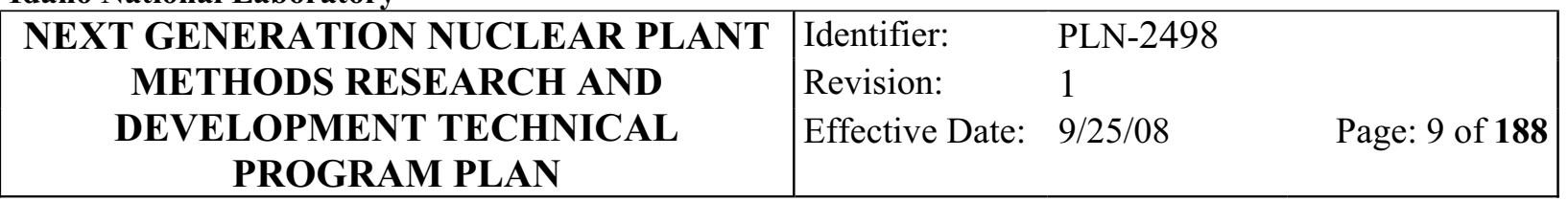

have to operate at a derated power condition as a function of the magnitude of the prescribed safety factor.

\subsection{Mission of the Design and Safety Methods Validation Program}

The mission of the Design and Safety Methods Validation Program is to develop and benchmark state-of-the-art analysis tools that will enable the vendors, the NRC, and DOE analysts to accurately calculate the core power distribution, core bypass, and peak outlet jet temperatures such that localized hot spots can be identified and either eliminated by design or quantified to a degree that licensing calculations can demonstrate the VHTR plant operation without challenging the safety margins, plant parameters, or figures-of-merit. Advanced tools for analyzing the VHTR are consistent with the new safety concerns inherent to the new design and system design requirements.

\subsection{Advantages of Advanced Analysis Tools}

Advanced analysis tools offer the following advantages over older legacy analysis tools:

- Reduced design uncertainty and risk because localized conditions can be calculated with a known uncertainty.

- Reduced design iterations and design costs because plant designers can more rapidly converge on their desired design configuration with greater certainty.

- An accelerated licensing process because the process of prescribing arbitrary safety factors is transformed to a process of using known quantities with quantified uncertainties.

- The capability of quantifying the safety and operational margins to optimal values for maximum outlet temperatures and maximum operational efficiencies.

\subsection{Economics of Using Advanced Analysis Techniques}

The NRC notes that U.S. utilities have been applying for power uprates to their operating licenses for some time (http://www.nrc.gov). One of the most common uprates falls in the category of stretch power uprates, which are typically up to $7 \%$ and are within the design capacity of the plant. The uprates have been achieved by using improved analysis tools to quantify and recapture margin. The actual value of the percentage increase in power that a plant can achieve and stay within the stretch power uprate category is plant-specific and depends on the operating margins included in the design of a particular plant. These uprates have produced major cost savings and improved plant output well in excess of the cost of developing and benchmarking LWR analysis tools.

For example, if the $600 \mathrm{MWt}$ NGNP could produce 5 to $10 \%$ more power or 630 to $660 \mathrm{MWt}$, then, based on current replacement power costs, the cost savings for the above improvements are at a minimum $\$ 210$ million, but have the potential to exceed $\$ 400$ million for only one $600 \mathrm{MWt}$ plant in replacement costs over the life of the plant. Such a cost savings more than justifies the cost of producing, validating, and benchmarking the advanced methods required to achieve the objectives of the NGNP Methods Plan, which may cost approximately $\$ 120$ million $^{\mathrm{g}}$ depending on the scope of work chosen for the methods evaluation effort.

g \$120 million is required to complete the entire scope of work outlined in the NGNP Methods Program, including software development, benchmark activities, and actual physical experiments. 


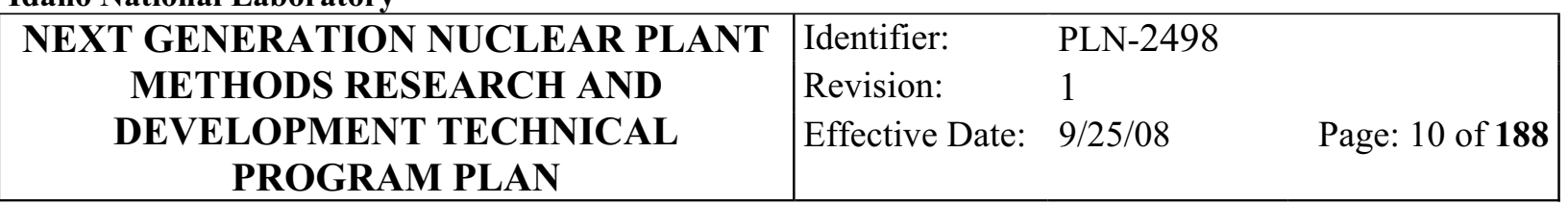

The NGNP Design and Safety Methods Validation Program will use the inherent accuracies of advanced analysis techniques over legacy analysis techniques to:

- Make the design analysis techniques more efficient and less costly

- Make the licensing analysis benchmarking and licensing process less time consuming and more quantifiable.

- Reduce the plant operational and safety margins between 5 and 10\% using benchmarked analysis tools and quantified uncertainties.

Areas for methods development and benchmarking include thermal-fluids modeling and experiments, reactor physics, core kinetics and fuel management modeling, and some nuclear data enhancements. These efforts will enable the NGNP plant designers to calculate plant safety margins and uncertainties, and reduce the effect of licensing assumptions, thus producing a safer, more cost-effective design. 


\begin{tabular}{|c|lll|}
\hline NEXT GENERATION NUCLEAR PLANT & Identifier: & PLN-2498 \\
METHODS RESEARCH AND & Revision: & 1 & \\
DEVELOPMENT TECHNICAL & Effective Date: & $9 / 25 / 08$ & Page: 11 of 188 \\
PROGRAM PLAN & & & \\
\hline
\end{tabular}

\section{RESEARCH AND DEVELOPMENT METHODOLOGY}

This chapter describes the overall methodology used to define the R\&D needed to produce the validated analytical tools required for the VHTR analysis. The implementation methodology for methods R\&D consists of eight interacting activities shown in Figure 1 and expanded to show other supporting activities in Figure 3, which are discussed in the remaining sections of this R\&D plan:

- Selection of the most challenging scenarios together with the dominant phenomena in each (Section 3.1)

- Internal validation of the software tools and data required to calculate the NGNP behavior in each scenario (Section 3.3)

- External validation of the software tools via non-NGNP Project nuclear engineering community participation in international standard problems (Section 3.3)

- $\quad$ R\&D performed through Generation IV International Forum (GIF)-member and NGNP Project collaborations centered in International Nuclear Engineering Research Initiatives (Section 3.3)

- R\&D performed through university and NGNP Project collaborations centered in Nuclear Engineering Research Initiatives or GIF Project Management Board agreements (Section 3.3)

- Software development when validation findings show that certain models are inadequate (Section 3.4)

- Analysis of the operational and accident scenarios (Section 3.5)

- Review of the global process and process ingredients using experts outside the program (Section 3.5).

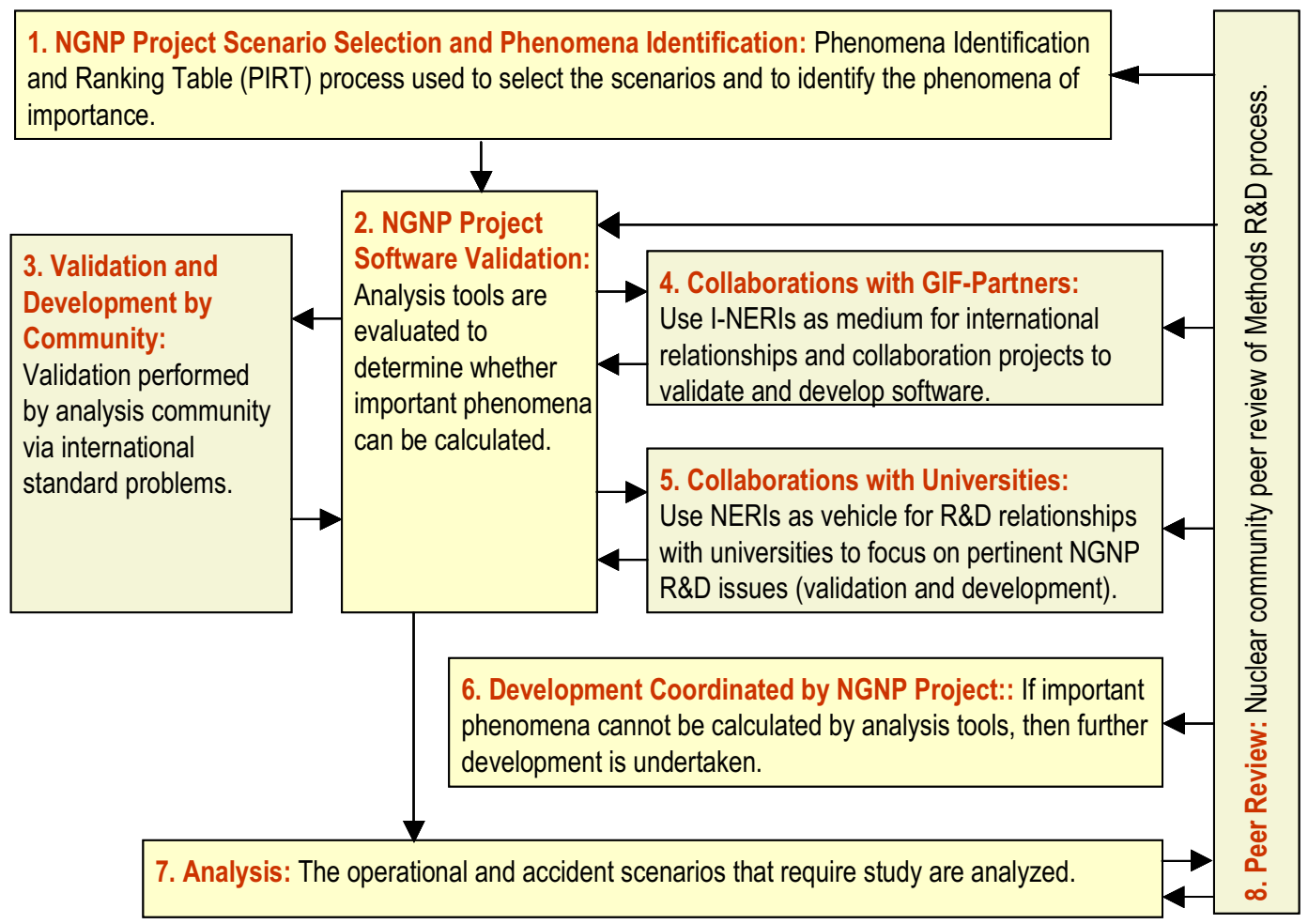

Figure 3. Expanded description of methods R\&D process. 


\begin{tabular}{|c|lll|}
\hline NEXT GENERATION NUCLEAR PLANT & Identifier: & PLN-2498 & \\
METHODS RESEARCH AND & Revision: & 1 & \\
DEVELOPMENT TECHNICAL & Effective Date: & $9 / 25 / 08$ & Page: 12 of 188 \\
PROGRAM PLAN & & & \\
\hline
\end{tabular}

\subsection{Scenario Identification and Phenomena Identification and Ranking Tables}

In accordance with Figure 3 and the project schedule, this section describes task work divided into two phases: Pre-vendor/design selection, and Post-vendor/design selection. During Phase 1, scenarios are identified for plant duty cycle events pertaining to two generic designs: a pebble-bed reactor design and a prismatic reactor design. Phenomena are identified for these events for these two designs at a high level to provide input to the planning for the R\&D program. In particular, the ranking of the phenomena allows the prioritization of model development for the design and safety tools and in the planning of experimental facilities and experiment matrices. Phase 1 objectives include the screening of existing facilities that have the potential for contributing validation data to the methods R\&D process outlined in Figure 3 as well as the screening of existing data. Where the data are not publically available, in particular international data, interactions with the various organizations will be initiated and pursued. Once vendor/design selection has occurred, then Phase 2 will be implemented in close coordination with the plant vendor. During Phase 2, input will be provided not only to the R\&D Plan, but also to aid in the licensing effort and licensing interactions with NRC. Two stages are envisioned: phenomena Identification and Ranking Tables (PIRTs) will be generated during the conceptual design stage with the conceptual design and, as the design proceeds to the final design stage, finalized PIRTs will be generated for the final design. This will all be closely collaborated with the vendor.

\subsubsection{Scenario Identification}

To show that the VHTR meets all safety requirements, proven analysis capability must be available to model not only the operational conditions, but also the accident conditions. Various aspects of the core behavior must also be modeled, including:

- Operational characteristics of the TRISO fuel throughout the VHTR's life cycle, e.g., the fuel temperature profile, the migratory characteristics of the fuel kernel within the fuel microsphere, the shrinkage and swelling of the various pyrolytic carbon coatings, and the stress distributions in the coating layers. All of these operational characteristics are modeled numerically in the PARFUME software (Miller, Petti, Maki, and Knudson 2004; Petti, Hobbins, Kendall, and Saurwein 2005)

- Fuel power distribution as a function of exposure in both the fuel compacts or balls pebbles and in the microspheres

- Thermal fluid conditions during both operating conditions and transient conditions, including the fuel temperature profiles and also the maximum temperatures of plant structural members such as the core barrel, core support plate, and vessel wall

- Mixing characteristics of the fluid inventory in the plena: the lower plenum during operating conditions since the hot exit gases are delivered to the turbine and both plena during a loss-of-forcedflow scenario

- Potential for air ingress and graphite oxidation subsequent to a loss-of-coolant accident (LOCA)

- Fission product release and transport as a function of projected TRISO fuel failure rates.

The highest-priority R\&D for fiscal years 2007, 2008, and 2009 is aimed at properly calculating the thermal-fluid conditions in the "hot channels" and mixing them in the lower plenum during normal operation. 


\begin{tabular}{|c|lll|}
\hline NEXT GENERATION NUCLEAR PLANT & Identifier: & PLN-2498 \\
METHODS RESEARCH AND & Revision: & 1 & \\
DEVELOPMENT TECHNICAL & Effective Date: & $9 / 25 / 08$ & Page: 13 of 188 \\
PROGRAM PLAN & & & \\
\hline
\end{tabular}

The full spectrum of possible accident scenarios of importance is not fully defined, since it is dependent on the presently undefined VHTR design, but, on the basis of the work done to license the Fort St. Vrain (FSV) and AVR reactors, it is known that the following scenarios, at a minimum, must be analyzed, as indicated in the FSV Final Safety Analysis Report (FSAR):

- Anticipated operational occurrences:

a. Main loop transient with forced core cooling

b. Loss of main and shutdown cooling loops

c. Accidental withdrawal of a group of control rods followed by reactor shutdown

d. Small break LOCA $\left(\sim 1\right.$ in. $^{2}$ area break).

- Design basis accidents (assuming that only "safety-related" systems can be used for recovery):

a. Loss of heat transport system and shutdown cooling system (similar to scenario $1 \mathrm{~b}$ above)

b. Loss of heat transport system without control rod trip

c. Accidental withdrawal of a group of control rods followed by reactor shutdown

d. Unintentional control rod withdrawal together with failure of heat transport systems and shutdown cooling system

e. Transient without scram

f. Earthquake-initiated trip of heat transport system

g. LOCA event in conjunction with water ingress from failed shutdown cooling system

h. Large break LOCA

i. Small break LOCA.

On the basis of the experience of gas-cooled reactor designers and experimentalists (Ball 2003 and Krüger et al. 1991), scenarios 2a and 2g, hereafter referred to as the Pressurized Conduction Cooldown (PCC) scenario and the Depressurized Conduction Cooldown (DCC) scenario, respectively, are considered the most demanding and most likely to lead to maximum vessel wall and fuel temperatures. Hence, first-cut R\&D specifications are based on calculation of the hot-channel temperatures and mixing characteristics in the lower plenum during normal operation, and the PCC and the DCC scenarios from the accident envelope.

\subsubsection{Phenomena Identification and Ranking Tables}

The PIRT process entails carefully identifying the most demanding scenarios, followed by prioritizing the phenomena that are found in the most demanding scenarios. Key phenomena are those exerting the most influence on the path taken during the most demanding scenarios. Thus, as discussed in the previous paragraphs, the key phenomena for the PCC and DCC scenarios, or most "highly ranked" phenomena, are those that exert the greatest influence on the peak core temperatures and peak vessel wall temperatures (Gas Reactor Technology Course; ANS 2003). During normal operation other key phenomena such as stresses or irradiation-induced dimensional changes may be important.

A detailed PIRT cannot be completed because the specific VHTR design has yet to be selected. However, during the interim, a first-cut PIRT has been used as a guide for the initial R\&D work and planning for both block-type and pebble-bed-type gas-cooled reactors. The first-cut PIRT is based on observations from seasoned gas-cooled reactor experts and engineering judgment; these factors were used by a team assembled to define the first PIRT for the prismatic and PBRs - the first-cut PIRT. The first-cut PIRT is given in Appendix A and is documented in detail in Lee, Wei, and Schultz et al 2005. The results 


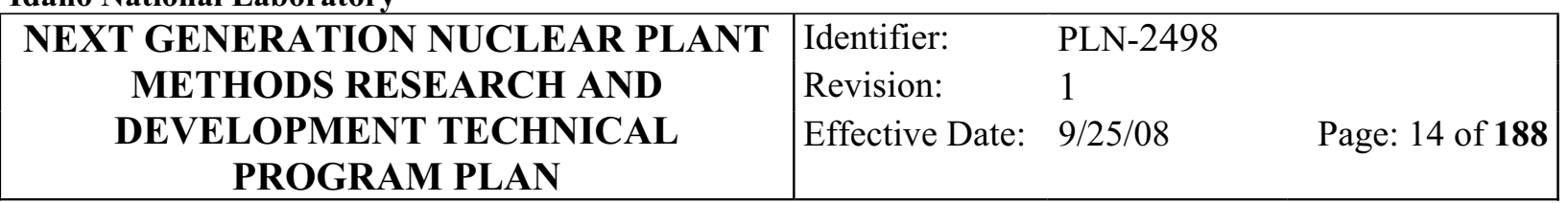

of the first-cut PIRT for steady-state operation, PCC, and DCC scenarios are given in Table 1 for only the upper and lower plena, the core, and the reactor cavity cooling system (RCCS). Terminology used in the table is described in the following paragraphs and also in Appendix A.

Table 1. PIRT results for normal operation, PCC, and DCC scenarios.

\begin{tabular}{|c|c|c|c|c|}
\hline Scenario & Upper Plenum & Core & RCCS & Lower Plenum \\
\hline $\begin{array}{l}\text { Normal } \\
\text { operation }\end{array}$ & $\begin{array}{l}\text { - Flow } \\
\text { distribution } \\
\text { - Pressure drop }\end{array}$ & $\begin{array}{l}\text { - Reactivity feedback behavior } \\
\text { - Core configuration (bypass) } \\
\text { - Pressure drop } \\
\text { - Heat transfer } \\
\text { - Flow distribution } \\
\text { - Power distribution } \\
\end{array}$ & $\begin{array}{l}\text { - Heat transfer at } \\
\text { operational } \\
\text { conditions } \\
\text { - Natural circulation in } \\
\text { cavity }\end{array}$ & $\begin{array}{l}\text { - } \text { Flow distribution } \\
\text { - Heat transfer } \\
\text { - Thermal striping } \\
\text { - Jet behavior }\end{array}$ \\
\hline DCC & $\begin{array}{l}\text { - } \text { Mixing and } \\
\text { stratification } \\
\text { - Hot plumes } \\
\text { - Thermal } \\
\text { resistance of } \\
\text { structures }\end{array}$ & $\begin{array}{l}\text { - Thermal radiation and } \\
\text { conduction of heat across the } \\
\text { core } \\
\text { - Axial heat conduction and } \\
\text { radiation } \\
\text { - Natural circulation in the } \\
\text { reactor pressure vessel } \\
\text { - Air and water ingress } \\
\text { - Potential fission product } \\
\text { transport } \\
\text { - Power distribution } \\
\text { - Core configuration } \\
\text { - Decay heat } \\
\text { - Flow distribution } \\
\text { - Material properties } \\
\text { - Pressure drop }\end{array}$ & $\begin{array}{l}\text { - Laminar-turbulent } \\
\text { transition flow } \\
\text { - Forced-natural mixed } \\
\text { convection flow } \\
\text { - Heat transfer- } \\
\text { radiation and } \\
\text { convection in duct }\end{array}$ & $\begin{array}{l}\text { - Thermal mixing } \\
\text { and stratification } \\
\text { - Flow distribution } \\
\text { - Air ingress }\end{array}$ \\
\hline $\mathrm{PCC}$ & $\begin{array}{l}\text { - } \text { Mixing and } \\
\text { stratification } \\
\text { - Hot plumes } \\
\text { - Thermal } \\
\text { resistance of } \\
\text { structures }\end{array}$ & $\begin{array}{l}\text { - Thermal radiation and } \\
\text { conduction of heat across the } \\
\text { core } \\
\text { - Axial heat conduction and } \\
\text { radiation } \\
\text { - Natural circulation in the } \\
\text { reactor pressure vessel } \\
\text { - Power distribution } \\
\text { - Core configuration } \\
\text { - Decay heat } \\
\text { - Flow distribution } \\
\text { - Material properties } \\
\text { - Pressure drop }\end{array}$ & $\begin{array}{l}\text { - Laminar-turbulent } \\
\text { transition flow } \\
\text { - Forced-natural mixed } \\
\text { convection flow } \\
\text { - Heat transfer- } \\
\text { radiation and } \\
\text { convection in duct }\end{array}$ & $\begin{array}{l}\text { - Thermal mixing } \\
\text { and stratification } \\
\text { - Flow distribution }\end{array}$ \\
\hline
\end{tabular}




\begin{tabular}{|c|lll|}
\hline NEXT GENERATION NUCLEAR PLANT & Identifier: & PLN-2498 \\
METHODS RESEARCH AND & Revision: & 1 & \\
DEVELOPMENT TECHNICAL & Effective Date: & $9 / 25 / 08$ & Page: 15 of 188 \\
PROGRAM PLAN & & & \\
\hline
\end{tabular}

\subsubsection{Mixing}

Mixing refers to the degree to which coolant of differing temperatures entering a region mixes to produce a uniform temperature. In the upper and lower plena, mixing is a 3-D phenomenon and a function of a number of variables. In the upper plenum, where it is identified as important in the PCC scenario, mixing occurs during natural convection as helium moves upward through the hottest portion of the core while cooler helium moves downward through the bypass and the cooler regions of the core. In the outlet plenum, mixing occurs between the bottom of the core and the turbine inlet during normal operation. A preliminary calculation of the temperature variation in the lower plenum is shown in Figure 4, where gas temperature variations are shown to exceed $100^{\circ} \mathrm{C}$. Although the specification for temperature variation at the turbine inlet has not been set, it is thought that the helium temperature variation at the turbine inlet must be less than $\pm 20^{\circ} \mathrm{C}$. It has also been seen that helium has a surprising resistance to thorough mixing (Ball 2004; based on experience of Kunitoni et al. 1986) and that the temperature in the core outlet jet can vary over a considerable range, particularly since the bypass flow may vary between 10 and $25 \%$. It is therefore likely that special design features will be required to ensure good mixing and minimal thermal streaking from the lower plenum to the turbine inlet.
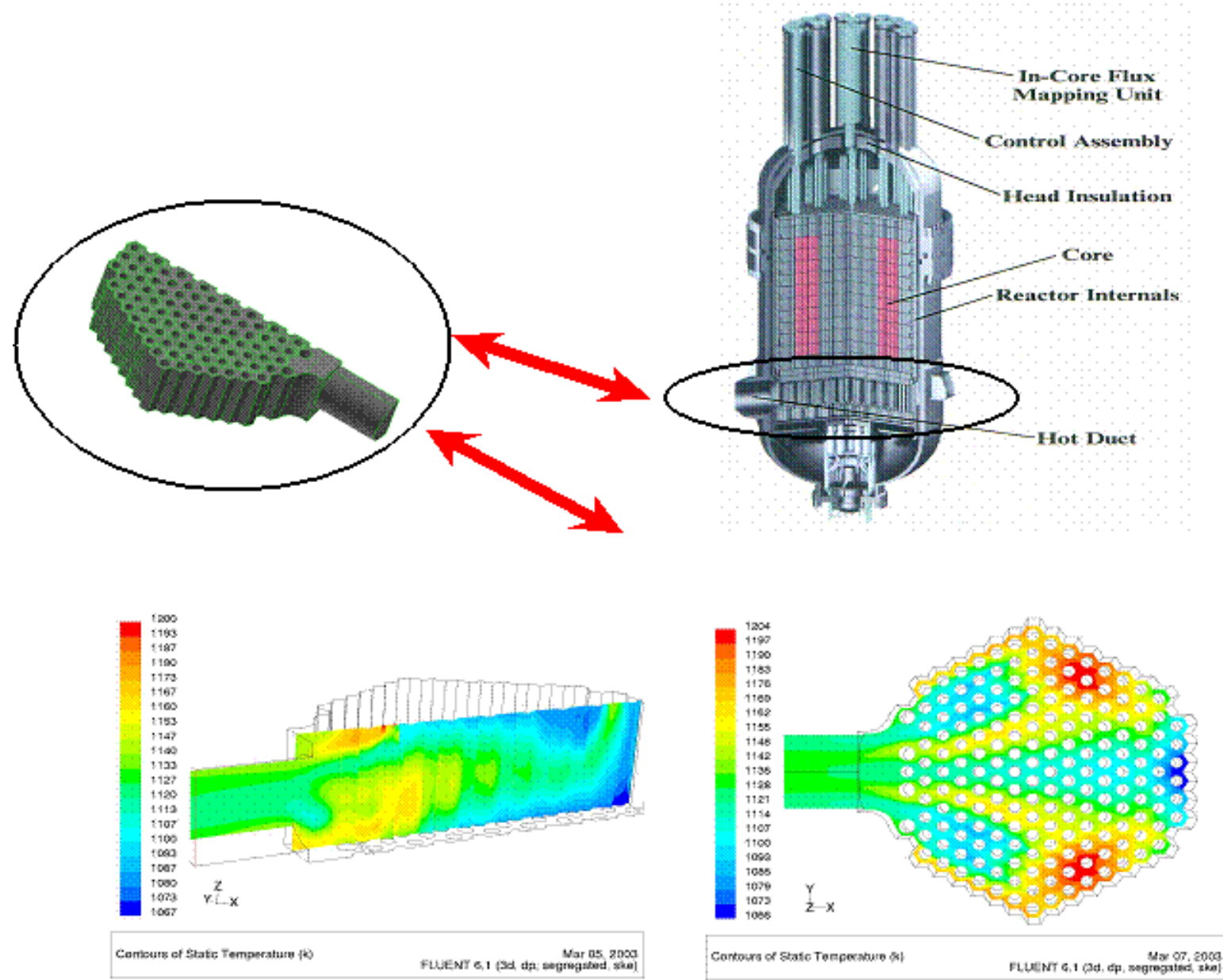

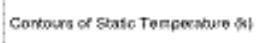

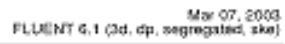

Figure 4. Computational fluid dynamics calculation of mixing in lower plenum (Courtesy of Fluent Corp). 


\begin{tabular}{|c|lll|}
\hline NEXT GENERATION NUCLEAR PLANT & Identifier: & PLN-2498 \\
METHODS RESEARCH AND & Revision: & 1 & \\
DEVELOPMENT TECHNICAL & Effective Date: & $9 / 25 / 08$ & Page: 16 of 188 \\
PROGRAM PLAN & & & \\
\hline
\end{tabular}

\subsubsection{Bypass}

Bypass flows through the reflector regions in both pebble-bed and block reactors and between the blocks in a block-type reactor. Because the quantity of bypass flow is a direct function of the bypass area, which in turn is a function of the temperature distribution, fluence, and graphite properties, the influence of the bypass on the core temperature distribution may be significant.

\subsubsection{Neutronic Behavior}

The current VHTR design candidates have a somewhat harder thermal neutron spectrum than standard LWRs, a more complex fuel geometry, and a fuel cycle with two to three times the burnup. At the very high burnups expected for the VHTR, the higher isotopes of plutonium contribute a significant amount of fission energy, but the cross-section information available from the current nuclear databases for ${ }^{240} \mathrm{Pu},{ }^{241} \mathrm{Pu}$, and ${ }^{242} \mathrm{Pu}$ is in need of improved absolute accuracy. It is also crucial that a suitable suite of evaluated integral neutronics benchmark experiments be available for reactor physics code validation at the appropriate time during the R\&D process. These issues are discussed in detail in Chapter 4 .

In addition to improvements in the cross-section data to increase the accuracy of the neutronics calculations, the anticipated high burnup level and concomitant increase in plutonium inventories require improvements in cross-section processing methods. These improvements are needed in the treatment of resonances in the thermal energy range in graphite-moderated reactors where upscattering is significant. The inability to account properly for this effect leads to substantial errors in the harder spectrum of a graphite-moderated reactor. Another aspect of improving cross-sections is to better account for the heterogeneity on two scales in the VHTR: the fine scale from the fuel particles, and the coarse scale from the pebbles or fuel compacts. The improvement in cross-section generation will reflect enhanced resonance treatment through the use of an improved Dancoff factor. Furthermore, the neutronicallyoptically thin core regions and the surrounding reflectors imply that, in the preparation of data for use in few-groups whole-core models, the homogenization-condensation step must account for spectral interpenetration between zones. These issues and related matters pertinent to neutronics codes for VHTR analyses are discussed in detail in Chapter 5.

\subsubsection{Laminar-Turbulent Transition Flow and Forced-Natural Mixed Convection Flow}

Figure 5 shows a likely layout for the VHTR with the reactor pressure vessel and the vessel containing the intermediate heat exchanger and primary coolant system circulator sited below grade. During the PCC scenario in the core region and during both the PCC and DCC scenarios in the RCCS, there is the potential for having convective cooling in the transition region as shown in Figure 6, where an example is shown of convection flow regimes along the heater (reactor core) and cooler (heat exchanger providing ultimate heat sink) at various pressures in a simplified Reynolds-Rayleigh number map (Williams et al. 2003). Although Figure 6 was generated for a typical gas fast reactor core having hexagonal blocks with circular coolant holes, analogous behavior may occur in the VHTR in various locations and should be investigated. Because the convective cooling contribution is an important ingredient in describing the total heat transfer from the core and thus the ultimate peak core and vessel temperatures, these heat transfer phenomena are potentially important. 


\begin{tabular}{|c|lll|}
\hline NEXT GENERATION NUCLEAR PLANT & Identifier: & PLN-2498 \\
METHODS RESEARCH AND & Revision: & 1 & \\
DEVELOPMENT TECHNICAL & Effective Date: & $9 / 25 / 08$ & Page: 17 of 188 \\
PROGRAM PLAN & & & \\
\hline
\end{tabular}

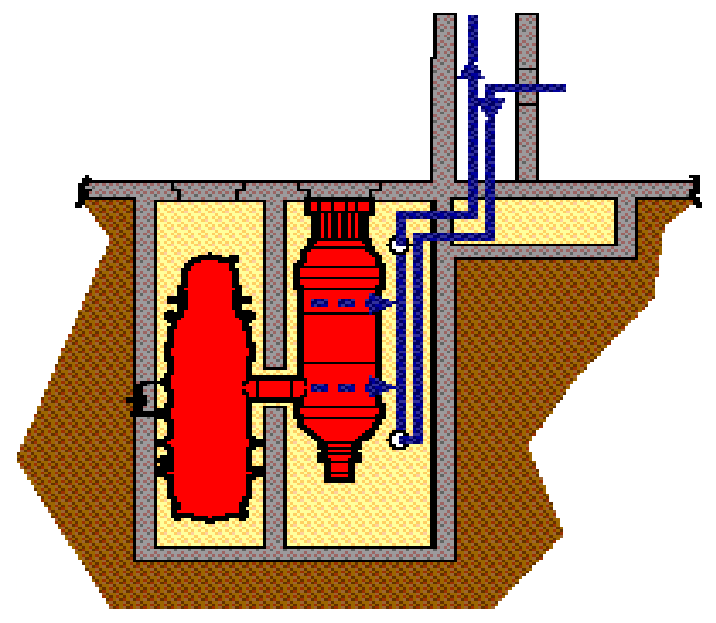

Figure 5. Reactor Cavity Cooling System Configuration

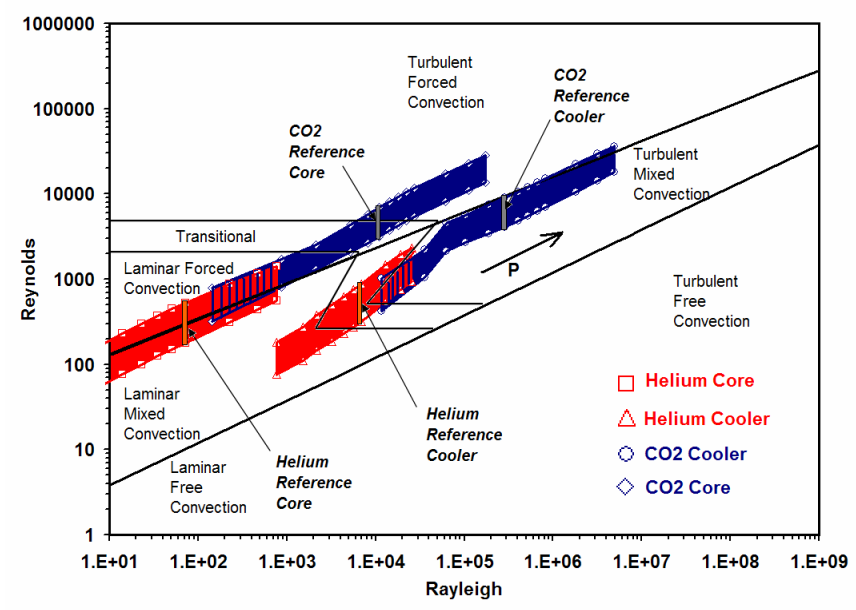

Figure 6. Convection flow regimes at various operating pressures for both helium and $\mathrm{CO}_{2}$ (from Williams, et al, 2003)

\subsubsection{Core Hot Channel Characterization}

The characteristics of the hottest cooling channels at operational conditions are considered a key calculational result since the hot channel temperature distribution defines the hottest initial condition for the fuel and surrounding materials. Hence preliminary CFD studies have been initiated and validation data are sought.

\subsubsection{Flow Distribution}

The distribution of the flow between the various coolant channels in a prismatic reactor (and the complementary behavior in both the upper and lower plena) are important to determine not only the warmest part of the core, but also the location of potential hot spots in the plena wall where hot plumes or jets might impinge.

\subsubsection{Air and Water Ingress}

For loss-of-coolant scenarios, such as the DCC, there is the potential, depending on the scenario assumptions, for air and water ingress into the core in perhaps harmful quantities. Air is present in the reactor cavity and will enter the core by diffusion in a DCC accident. Water is normally present in the air in the form of humidity, but it may enter the core in much greater quantities and a far greater potential effect on reactivity, if the shutdown cooling system suffers a pipe break.

\subsubsection{Fission Product Transport}

Fission product transport must be calculated for cases where some fraction of the TRISO fuel particles fail prior to or in conjunction with the DCC scenario and because certain fission products such as silver and palladium may diffuse through the TRISO coatings. Dust that may contain fission products must be tracked and accounted for using state-of-the-art calculational tools, particularly for the PBR. 


\begin{tabular}{|c|lll|}
\hline NEXT GENERATION NUCLEAR PLANT & Identifier: & PLN-2498 & \\
METHODS RESEARCH AND & Revision: & 1 & \\
DEVELOPMENT TECHNICAL & Effective Date: & $9 / 25 / 08$ & Page: 18 of 188 \\
PROGRAM PLAN & & & \\
\hline
\end{tabular}

\subsection{Analysis Tools and Data}

The analysis requirements (items 1 through 6 in Section 3.1.1) can only be achieved by using a suite of software tools and associated data libraries. For some calculational needs, more than one software tool may sometimes be used to achieve the calculational objective, each tool having a unique strength. The calculational process that satisfies the analysis requirements identified in Section 3.1 above is broken into seven steps, as shown in Figure 7. These seven steps are summarized in paragraphs a through $\mathrm{g}$ below; each paragraph item letter corresponds to a box on the flow chart shown in Figure 7.

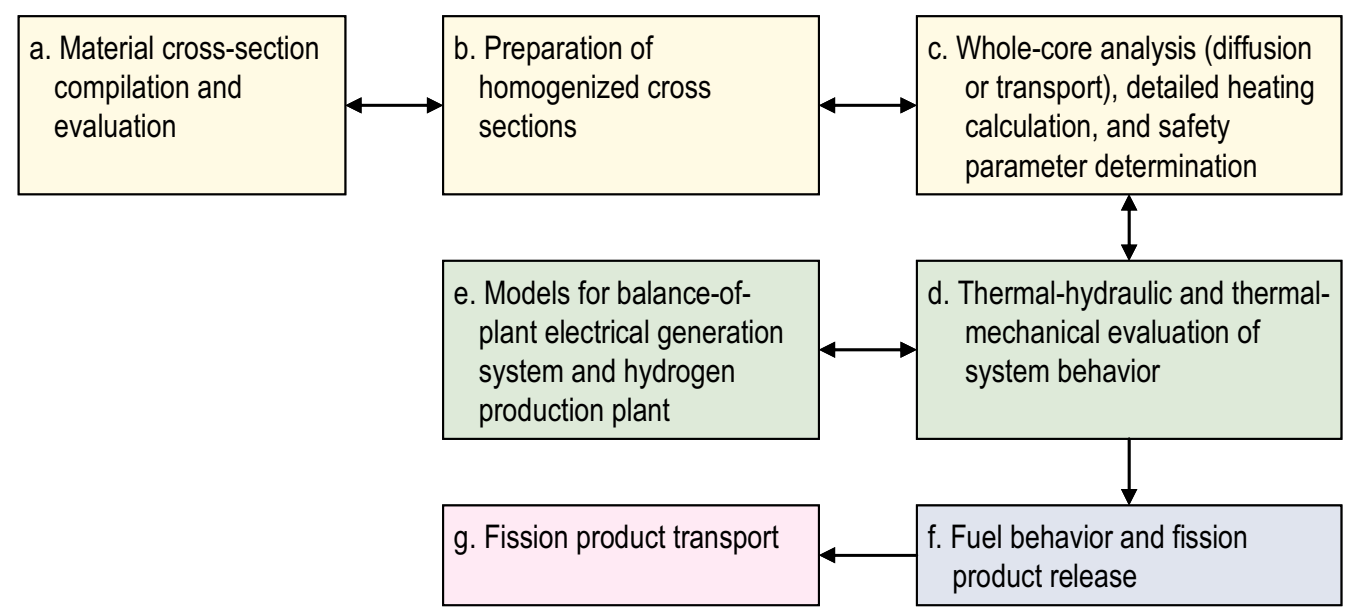

Figure 7. Calculation process.

1. Material cross section compilation and evaluation. Nuclear interaction cross sections are among the most basic fundamental engineering data required for design, licensing, and operation of nuclear systems. Compared to current LWRs, any of the proposed NGNP configurations will feature a somewhat harder neutron spectrum, a more complex fuel form, and two to three times greater burnup. Studies show that there is a near-term need for improved cross section measurements in certain neutron energy ranges for some isotopes to support the extensive computational modeling that will be required for the NGNP design, regardless of the specific basic reactor configuration that is ultimately selected. The isotopes ${ }^{240} \mathrm{Pu},{ }^{241} \mathrm{Pu}$, and ${ }^{242} \mathrm{Pu}$ are particularly important at high burnup. Improved cross section data are ultimately incorporated into the Evaluated Nuclear Data Files (ENDF) maintained by the U.S. National Nuclear Data Center. These data are subsequently processed to produce input libraries useful in reactor analysis software.

2. Preparation of homogenized cross sections. Before it can be used for a specific reactor application, ENDF data, as processed into a general format by NJOY or a similar tool, must be further processed into a case-specific form using local cell and assembly modeling codes. The basic physical data are processed for case-specific resonance shielding and then weighted with characteristic energy and spatial flux profiles generated from unit cell or super-cell models. This step is performed using software that approximates the neutron transport equation using PN or BN transport codes for the energy flux calculation and a 1-D or 2-D transport code for the spatial flux. (In the advanced lattice codes, spatial resolution is typically done using integral transport methods [collision probability or method of characteristics approaches.]) Software that will be initially evaluated for this function includes COMBINE, BONAMI/NITAWL, MICROX-2, WIMS-8, HELIOS, and DRAGON. An appropriate suite of codes will be implemented and validated according to accepted standards. The 


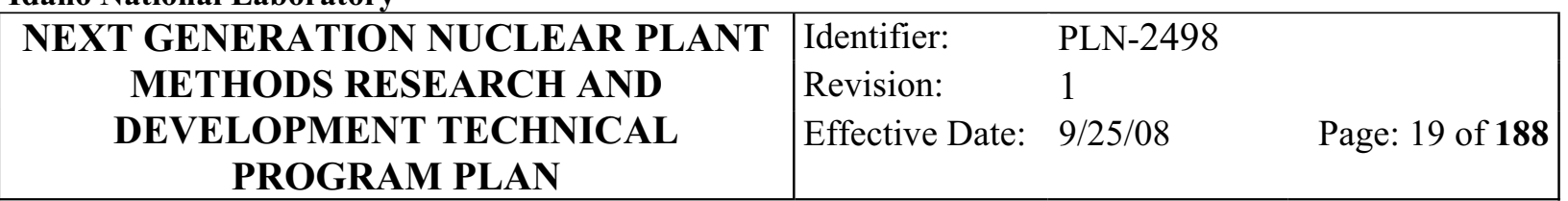

geometric aspects of this process are significantly different in the prismatic and pebble-bed concepts so two computational paths are shown in Figure 8. For additional assurance that the computational results obtained using diffusion theory codes are accurate, higher order deterministic transport methods should be employed to perform selective benchmark checks. Representative software that might serve this function is ATTILA, TWODANT, THREEDANT, or DORT/TORT. These transport packages are also used as part of the assembly cross-section preparation process.

3. Whole-core analysis (diffusion or transport), detailed heating calculations, and safety parameter determination. Nodal diffusion-theory codes, such as DIF3D and the INL-developed code PEBBED, which is designed specifically for pebble-bed reactor simulation, will be the centerpiece production codes to perform NGNP reactor core analyses. Similarly, for the prismatic block cores, a nodal solver in hexagonal-z geometry, such as DIF3D or HEXPEDITE, will be used. Steady-state eigenvalues, energy and spatial flux profiles, reaction rates, reactivity changes (burnup and control rod movement), etc., will be calculated with the nodal diffusion-theory codes. Multigroup cross section data generated in the reactor assembly cross section preparation step (Step b above) will be provided to the nodal diffusion code.

The PEBBED code suite seamlessly integrates all the functions required of a fuel management analysis code, including online re-homogenization (to account for the spectrum interpenetration) and depletion modeling. At INL, the planned hexagonal-z analogue will use the same architecture, albeit the nodal solver will be a hexagonal-z solver. The possible choices for the solver are HEXPEDITE, PARCS, NESTLE, and The DIF3D-VARIANT, which code also contains a nodal transport option (VARIANT) based on the variational transport approach. To consider the power behavior as a function of fuel depletion, additional capabilities are required. This function is usually performed by the REBUS code in conjunction with DIF3D, whereas, it is internal to the PEBBED code for the pebble-bed reactor case. All of these software packages will be verified against alternate computational models, especially models based on the well known MCNP stochastic simulation (Monte Carlo) code as shown in the upper left center of Figures $8 \mathrm{a}$ and $8 \mathrm{~b}$, and various deterministic approaches. In addition, all of the reactor physics models will be validated against various suitable experimental benchmarks.

A preliminary assessment of appropriate validation benchmarks pertinent to the current gas-cooled NGNP reactor concepts has in fact been completed by INL and ANL and more detailed benchmark evaluations are now underway. Output from the nodal diffusion codes will not only provide the steady-state operational physics parameters for each operational analysis conducted, but it will also be used as the initial condition for reactor kinetics calculations required as part of the overall system analyses performed in Steps d and e below. Spatial changes in flux and power level as functions of time during postulated transients, predicted by the kinetics module, will provide the energy source term required for the overall thermal-fluids systems code computations at each time step during each transient. This process permits full coupling of thermal and neutronics computations, consistent with modern practice for nuclear systems analysis. The NESTLE code, a subroutine in the RELAP5-3D systems analysis thermal-fluids code, will serve this purpose for the prismatic reactor concept. The CYNOD code, a time-dependent implementation of the PEBBED code, which has been coupled to the RELAP5-3D code, will be used for the pebble-bed concept.

4. Thermal-fluid and thermal-mechanical evaluations of system behavior. The fluid behavior, and interactions with the neutronics, will be calculated using a systems analysis code, or perhaps a coupled systems analysis/CFD code. Examples of two systems analysis codes and a CFD code are RELAP5-3D, GRSAC, and Fluent. In such a coupling, systems analysis software is used to perform calculations of the overall system behavior considering the interactions between all the parts, e.g., the 


\begin{tabular}{|c|lll|}
\hline NEXT GENERATION NUCLEAR PLANT & Identifier: & PLN-2498 \\
METHODS RESEARCH AND & Revision: & 1 & \\
DEVELOPMENT TECHNICAL & Effective Date: & $9 / 25 / 08$ & Page: 20 of 188 \\
PROGRAM PLAN & & & \\
\hline
\end{tabular}

core, the plenums, the hot exit duct, the turbine, and the remainder of the plant. CFD codes, such as Fluent, are used to calculate the detailed 3-D fluid behavior in a region of the reactor such as a plenum. In some cases, where one code has been validated extensively, it can be used for limited validation of a second code. An example of this is shown in Figure 8 where GRSAC may be used to partially validate RELAP5-3D. In addition to analyzing the fluid behavior under a spectrum of operating and accident conditions, the thermal-fluid tools also will be used to investigate the significance of material geometric tolerance variations due to manufacturing, thermal responses, and irradiation effects such as graphite swelling. The need to examine factors that affect thermalmechanical influence on fluid and heat transfer behavior will be included in the tool selection and evaluation process.

5. Models for balance-of-plant electrical generation system and hydrogen production plant. The behavior of the balance-of-plant systems will be modeled using a systems analysis code such as RELAP5-3D or ASPEN. ${ }^{\mathrm{h}}$ The balance-of-plant models are important to include in the analysis process to account for the important interactions that affect the system efficiency during normal operational conditions, but also to account for the equipment interactions that may lead to undesirable conditions such as turbine over-speed, loss of net positive suction head for auxiliary systems, or oscillatory conditions that may lead to equipment damage. Interactions between the reactor system and its balance-of-plant components lead to boundary conditions that will determine whether fueldamaging conditions are likely (see item f).

6. Fuel behavior and fission product release. The performance of fuel particles under irradiation is modeled to determine whether fuel failure will occur with the subsequent release of fission products and whether subsequent migration of fission products throughout the system must be considered. The INL software designed to perform this function is called PARFUME. In addition to the physical description of the fuel, an operation history generated by physics and thermal analysis codes (consisting of fuel temperature, burnup and fast neutron fluence) is used as input to PARFUME. The code models the mechanical and physicochemical behavior of the fuel and calculates the fraction of the fuel particle inventory that may fail. Several potential failure mechanisms are analyzed, including cracking of structural particle layers, debonding of the inner pyrolytic carbon layer from the silicon carbide ( $\mathrm{SiC}$ ) layer, buildup of internal fission gas pressure, kernel migration (amoeba effect) to the $\mathrm{SiC}$ layer, and thinning of the $\mathrm{SiC}$ layer by fission product interactions. PARFUME also calculates the fraction of selected fission product gases released from failed particles and from fission of uranium contamination in the matrix material surrounding the fuel particles. Calculation of the release of selected metallic fission products is currently under development. The fuel and fission product modeling activities are described in Petti, Hobbins, Kendall, and Saurwein (2005) and Miller, Petti, Maki, and Knudson (2004).

7. Fission product transport. If a loss-of-coolant accident has occurred, such that the fission products may migrate or be impelled into the confinement/containment building with perhaps subsequent release to the environment, the final calculational step is the prediction of the fission product movement into the environment and its environmental distribution. The software tool that will most likely be used to calculate fission product transport is MELCOR.

The process described in items a through g above is shown in the flow chart (Figure 7). The complete calculation process illustrated in Figure 7 is only exercised in its entirety for a few scenarios. Most scenarios would require the use of only a fraction of the calculations represented in Steps a-e. For example, scenarios that do not include a loss of coolant, such as a pipe break, usually would not require

h. Software used to model plant processes for multiple industries. ASPEN may be used to model a reactor balance-of-plant. 


\begin{tabular}{|c|lll|}
\hline NEXT GENERATION NUCLEAR PLANT & Identifier: & PLN-2498 \\
METHODS RESEARCH AND & Revision: & 1 & \\
DEVELOPMENT TECHNICAL & Effective Date: & $9 / 25 / 08$ & Page: 21 of 188 \\
PROGRAM PLAN & & & \\
\hline
\end{tabular}

calculation of fission gas transport (Step g). In addition, if the neutronics has been thoroughly calculated for the reactor system operating condition (Steps a-c), then a multitude of reactor system calculations can be performed using the evaluated reactor power state at time zero, and, hence, calculations made in Steps a through $\mathrm{c}$ may only need to be performed once for a desired operating condition. Thereafter, for such scenarios that assume reactor scram (requiring no reactor kinetics), a multitude of calculations can be performed using only the software tools developed for Steps $\mathrm{d}$ and e.

Figures $8 \mathrm{a}$ and $8 \mathrm{~b}$ identify the software associated with each step in Figure 7 for the pebble-bed and prismatic block reactors, respectively.

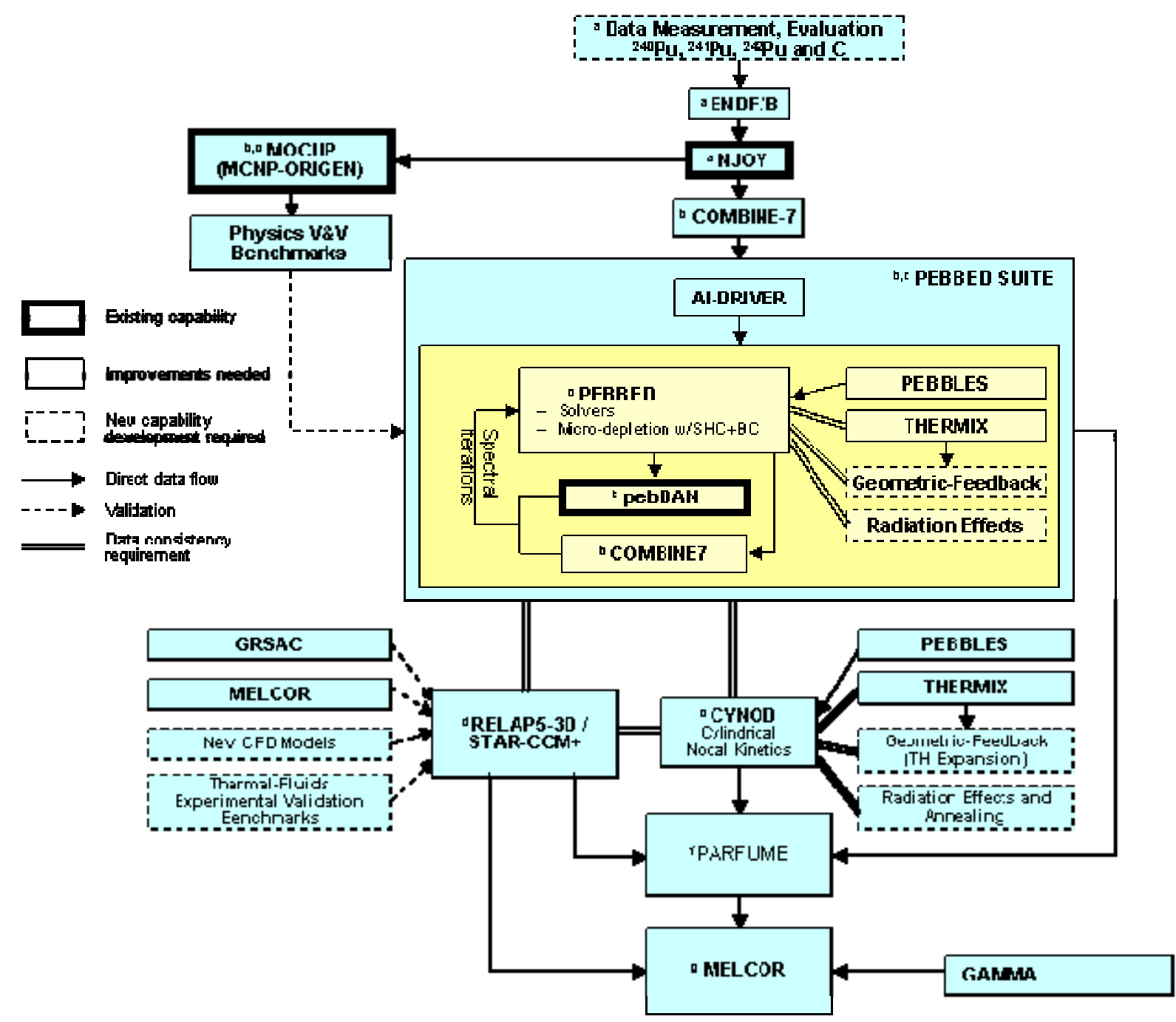

Figure 8a. Application of process to pebble-bed candidate designs for NGNP with applicable software. 


\begin{tabular}{|c|lll|}
\hline NEXT GENERATION NUCLEAR PLANT & Identifier: & PLN-2498 \\
METHODS RESEARCH AND & Revision: & 1 & \\
DEVELOPMENT TECHNICAL & Effective Date: & $9 / 25 / 08$ & Page: 22 of 188 \\
PROGRAM PLAN & & & \\
\hline
\end{tabular}

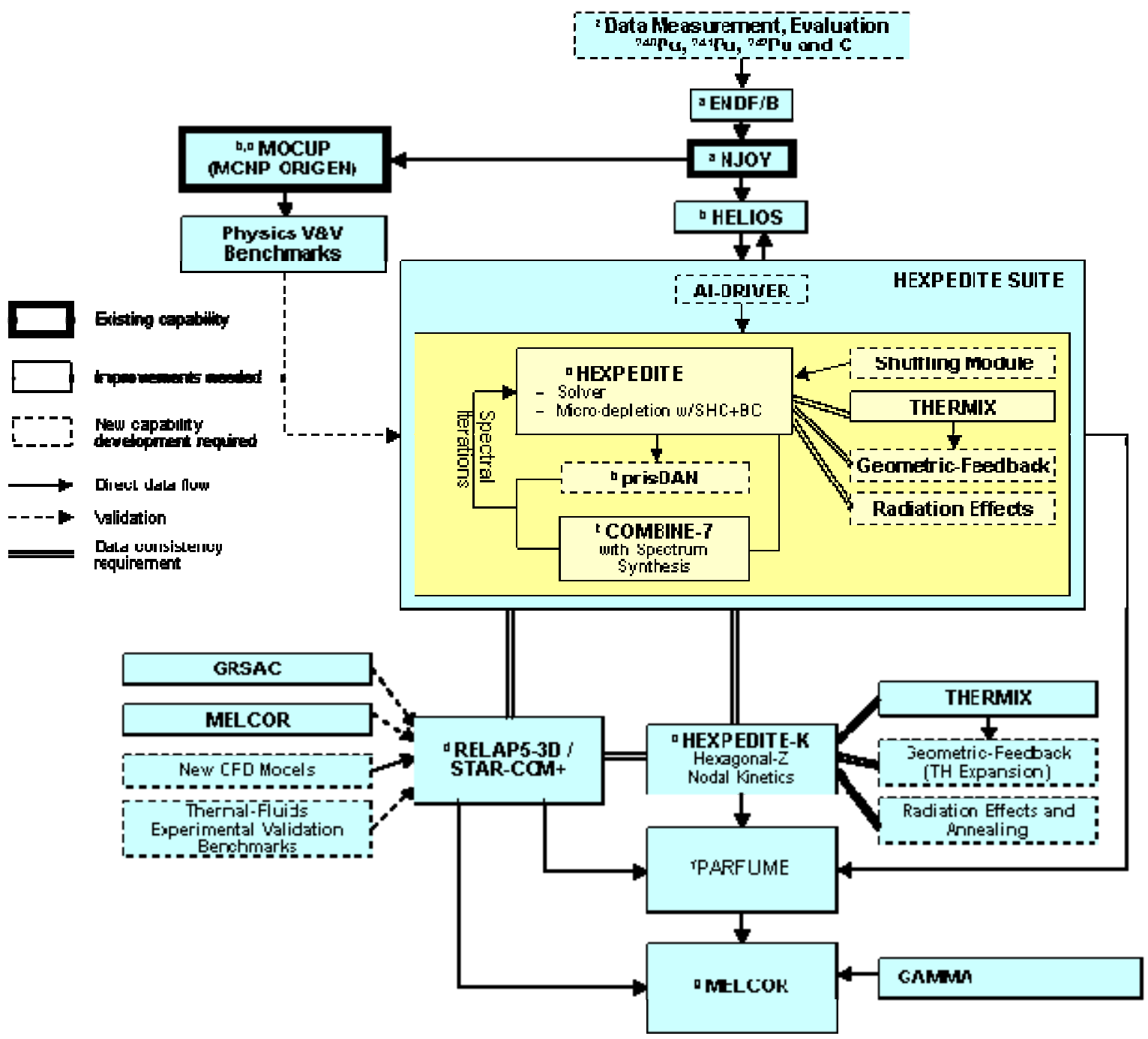

Figure 8b. Application of process to prismatic candidate designs for NGNP with applicable software.

\subsection{Verification}

Practices and procedures are divided into several categories to indicate the goal and intent of each. These categories include Code Verification, Code and Calculation Documentation, Reduction of Numerical Error, Quantification of Numerical Uncertainty, and Calculation Validation. Quantification of numerical uncertainty is discussed in some detail in Johnson et al. (2006). Validation, including calculation validation, is discussed in Section 3.4.

\subsubsection{Code Verification}

Code verification involves the determination of coding correctness (Roache 1998), a process separate from calculation verification (the quantification of numerical uncertainty). INL recognizes that the analysis software that will be applied to reactor safety analyses will already have been subjected to a variety of code verification tests. What will be required is documentation of these tests. 


\begin{tabular}{|c|lll|}
\hline NEXT GENERATION NUCLEAR PLANT & Identifier: & PLN-2498 \\
METHODS RESEARCH AND & Revision: & 1 & \\
DEVELOPMENT TECHNICAL & Effective Date: & $9 / 25 / 08$ & Page: 23 of 188 \\
PROGRAM PLAN & & & \\
\hline
\end{tabular}

Only tests that exercise the options used in the particular computations need to be documented. The tests as a suite must be designed to exercise all the terms in the governing partial differential equations. For example, it is not adequate to only test the code on linearly varying solutions such as planar Couette flow, since this solution does not exercise vertical convection terms and others. The most complete and convincing type of code verification test uses the Method of Manufactured Solutions or MMS (Roache 1998), but this will not be required. If MMS is not used, it will probably be necessary to use a suite of test problems to demonstrate code correctness. For all of these problems, the observed rate of discretization error convergence should be documented and compared to a theoretical value for the discretization algorithms employed. If it is not, then more stringent requirements will be enforced during calculation certification (Johnson et al. 2006). The code verification must also include some data on the effect of iterative convergence criteria on numerical results (see Section 3.3.3, "Reduction of Numerical Error" for details.)

\subsubsection{Code and Calculation Documentation}

Software that is used for nuclear reactor safety analysis must be described in detail in code documentation. Such documentation should include describing equations used and their discretization as well as the basics methods used to obtain a solution. The truncation error and its formal order or accuracy should be given. The code documentation must include all details of implementation of the turbulence models used in calculations, e.g., turbulence models for CFD software. The code documentation should be available for reference to reviewers who must review the associated calculational results.

For each calculation performed and submitted as a safety analysis, assumptions must be listed along with the details of the methods and models used. Other details, including but not limited to boundary and initial conditions, model constants (parameters), and other relevant information must also be provided. Options not used in the calculation need not be documented.

\subsubsection{Reduction of Numerical Error}

The reduction of numerical error is clearly a desirable objective for numerical calculations. Lessons have been learned about what not to do when using computational techniques for numerical analysis. These lessons-learned have been canonized in the requirements for manuscripts submitted to well known journals such as the ASME Journal of Fluids Engineering. It therefore seems prudent to apply them to relevant software to reactor safety analysis. Examples of such requirements are those given in the ASME Journal of Fluids Engineering "Statement on Numerical Accuracy." Details regarding the philosophy and meaning of the various key points are discussed in Johnson et al. (2006). Examples of the content include requirements that (a) methods must be at least second order accurate in space, (b) grid independence or convergence must be established, and (c) in transient calculations phase error must be assessed and minimized.

Grid independence is the process of refining the grid from the starting point until numerical results stop changing or change by negligible amounts. Theoretically, the results will continue to change until the grid spacing approaches zero. The precision of the machine, however, will halt this process at a finite grid spacing. This is sometimes referred to as achieving machine zero (of the residuals). Not only is the process of obtaining grid independence important to reducing numerical errors, it is also a good way to obtain estimates of numerical uncertainty (see "Quantification of Numerical Uncertainty" in Johnson et al. 2006).

Iterative convergence relates to the number of iterations required to obtain residuals that are sufficiently close to zero, either for a steady-state problem or for each time step in an unsteady problem. 


\begin{tabular}{|c|lll|}
\hline NEXT GENERATION NUCLEAR PLANT & Identifier: & PLN-2498 \\
METHODS RESEARCH AND & Revision: & 1 & \\
DEVELOPMENT TECHNICAL & Effective Date: & $9 / 25 / 08$ & Page: 24 of 188 \\
PROGRAM PLAN & & & \\
\hline
\end{tabular}

This error is in addition to the numerical error associated with the truncation error terms. Because of the well-known and unacceptable sensitivity of some commercial codes to the iteration tolerance and the too lax default tolerance, the final calculations must determine this effect. At least two levels of iteration tolerance must be shown and the sensitivity presented. For example, if results for a solution functional $f$ are presented using a default iteration tolerance of (say) $10^{-3}$ reduction in residual from the initial condition, as required in Freitas et al (2003), then another calculation with $10^{-4}$ will be required, and the sensitivity f' 10 will be stated as the normalized \% change in $f$ per decade of change in iteration tolerance.

$f_{10}^{\prime}=\Delta f=\left[f\left(10^{-4}\right)-f\left(10^{-3}\right)\right] / f_{\text {norm }} \times 100 \%$

The normalization can be based on $f$ norm $=f\left(10^{-3}\right)$ when divides by near zero are not a problem, otherwise by another appropriate normalization. The final test of sufficient tightness of the iterative tolerance will be the acceptability of the final results based on estimation of numerical uncertainty and validation metrics.

For transient calculations, the same convergence criterion should apply as for spatial convergence (grid independence). The time step should be refined until negligible change is obtained. Also, though not required by the Journal of Fluids Engineering, it is recommended that the time-wise discretization scheme should be second-order accurate or better. While there are other practices to reduce numerical error, the above will constitute the required practices for rector safety analysis at the present time. Other practices that reduce numerical error are certainly allowed and even encouraged.

\subsection{Validation}

Whether or not software is adequate for performing best-estimate VHTR analyses is determined using both "top-down" and "bottom-up" evaluations, as summarized in Figure 9 and described in this section.

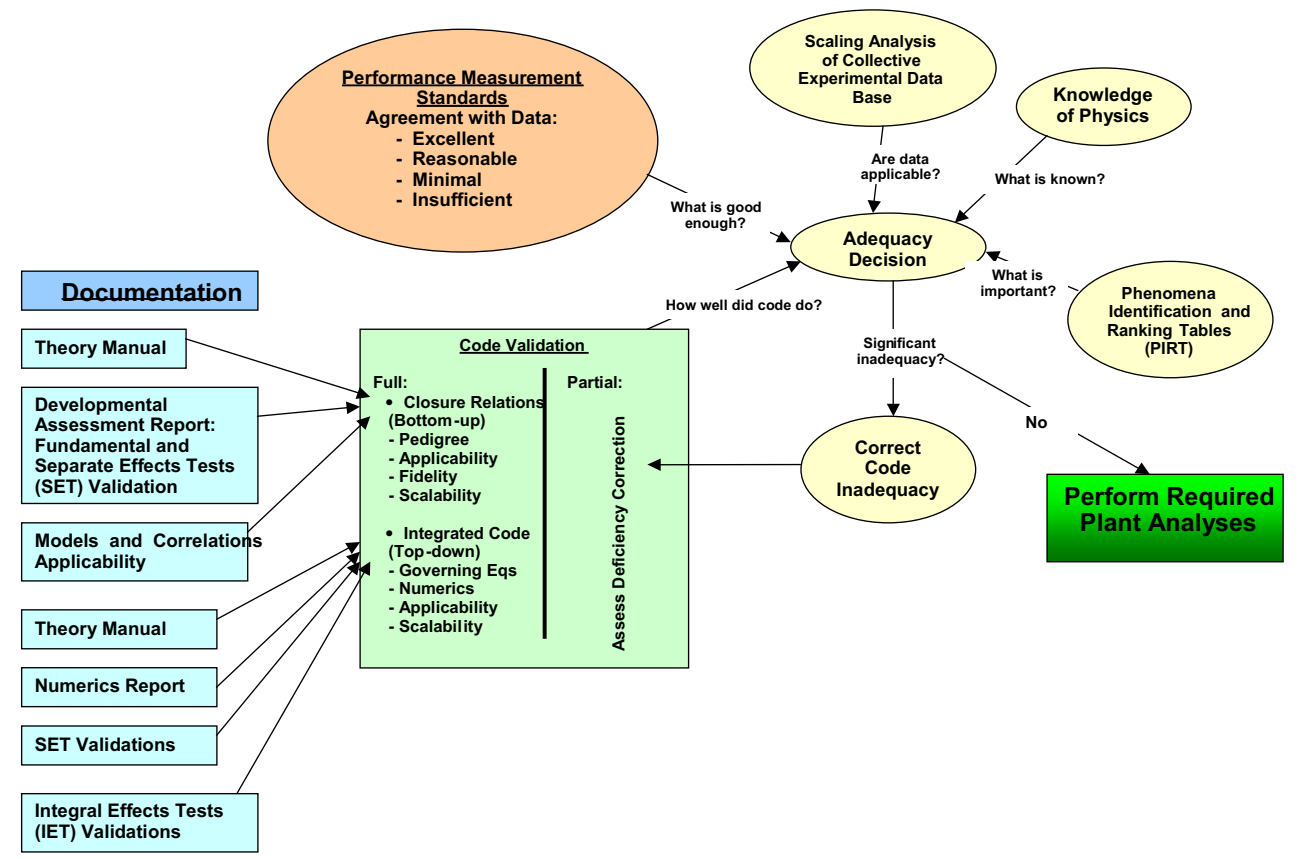

Figure 9. VHTR system design software-elements of adequacy evaluation and acceptance testing practices. 


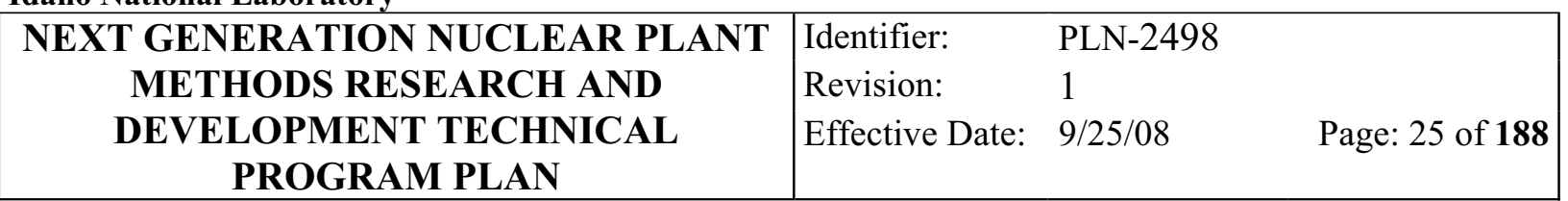

\subsection{1 "Bottom-Up" Code Adequacy}

Bottom-up evaluation of code adequacy entails examination of four features: the pedigree, applicability, fidelity, and scalability of the code under consideration.

The pedigree of a systems code consists of its history, its development procedures, and the basis for each correlation that is used in the code. Any correlations, data sources, and approximations used in the code must be documented in textbooks, laboratory reports, papers, etc. The uncertainty data used to bound the correlation(s), data, and approximations such as instrumentation and data system uncertainties must be included in the documentation. The basis for the uncertainties should be traceable and reproducible. The assumptions and limitations of the models must be known and documented.

The applicability of a systems code depends on the range of use of each of its correlations, data, and approximations. Those ranges must be documented and referenced. Finally, the range of applicability claimed in the code manual should be consistent with the pedigree, or, if a greater range is claimed, the justification for the increase in range must be reported.

The fidelity of a systems code means the degree to which the code's predictions agree with physical reality. High fidelity requires that the mathematical models and correlations used in the code are not altered in an ad-hoc manner from their documented formulation. A code is validated when it is shown that the code's predictions of key parameters agree within allowable tolerances with experimental data. The validation effort should be complete for all the key phenomena in the events of interest. Finally, benchmarking studies may either supplement the validation effort or make up the validation effort if appropriate standards are available, e.g., comparison of code calculation with a closed form solution.

"Bottom-up" scaling stems from the need to:

- Build experimental facilities that model the desired full-scale system

- Closely match the expected behavior of the most important transient phenomena in the scenarios of interest

- Demonstrate the applicability of data from a scaled facility to a full-scale system and to defend the use of data from a scaled facility in a code used to calculate the behavior of a full-scale system

- Relate a calculation of a scaled facility to a calculation of a full-scale system.

Scalability studies are usually performed to scale key parameters for a portion of the system behavior, not to correlate global system behavior. Therefore, scalability analyses consist of four steps: (1) isolate the "first-order" phenomena, (2) characterize the "first-order" phenomena, (3) convert the defining equations into nondimensional form, and (4) adjust the experimental facility conditions to give equivalent behavior with the full-scale system within the limitation of the facility (or nearly equivalent, based on nondimensional numbers that follow from Step 3).

As implied in the above discussion, "bottom-up" code adequacy techniques focus principally on closure relationships. Thus, the field equations used in the code must be correctly formulated and programmed. In addition, the field equations must be reviewed by the scientific community, and its agreement on the correct formulation and insertion of the governing equations in the code must be obtained. 


\begin{tabular}{|c|lll|}
\hline NEXT GENERATION NUCLEAR PLANT & Identifier: & PLN-2498 \\
METHODS RESEARCH AND & Revision: & 1 & \\
DEVELOPMENT TECHNICAL & Effective Date: & $9 / 25 / 08$ & Page: 26 of 188 \\
PROGRAM PLAN & & & \\
\hline
\end{tabular}

\subsection{2 “Top-Down” Code Adequacy}

The "top-down" approach for ensuring code adequacy focuses on the capabilities and performance of the integrated code. The top-down approach consists of four parts: numerics, fidelity, applicability, and scalability.

- Numerics. Evaluation of the numerical solution considers convergence, stability, and property conservation. ${ }^{i}$ Again, agreement by the scientific community on acceptable convergence, stability, and property conservation must be obtained.

- Fidelity. The fidelity of the code is demonstrated by performing thorough code assessments based on applicable integral-effects and separate-effects data. The data are part of an agreed-upon code assessment matrix constructed based on the transients of importance and the key phenomena for each phase of the transients.

- Applicability. The code must be shown to be capable of modeling the key phenomena in the system components and subsystems by conducting thorough validation studies. The key phenomena are identified in the PIRT.

The method to determine whether the code is capable of modeling key phenomena is to compare the calculation produced by the code to data that have known uncertainties. For example, "excellent" agreement between the code calculation and data is achieved if the calculated value is at all times within the data uncertainty band.

The degree of agreement between the code calculation and the data is generally divided into four categories as given in Table 2. A more rigorous definition is given by Schultz (1993). A code is considered adequate in applicability when it shows either excellent or reasonable agreement with the highly ranked phenomena (sometimes identified as the dominant phenomena) for a transient of interest. If the code gives minimal or unacceptable agreement, additional work must be performed, which may range from additional code development to additional analyses if needed to better understand the phenomena.

Table 2. Code adequacy identifiers

\begin{tabular}{|c|c|}
\hline Classifier & Description \\
\hline Excellent & $\begin{array}{l}\text { The calculation lies within or near the data uncertainty band at all times during } \\
\text { phase of interest. }\end{array}$ \\
\hline Reasonable & $\begin{array}{l}\text { The calculation sometimes lies within the data uncertainty band and shows the } \\
\text { same trends as the data. Code deficiencies are minor. }\end{array}$ \\
\hline Minimal & $\begin{array}{l}\text { Significant code deficiencies exist. Some major trends and phenomena are not } \\
\text { predicted. Incorrect conclusions may be drawn based on the calculation without } \\
\text { benefit of data. }\end{array}$ \\
\hline Unacceptable & $\begin{array}{l}\text { A significant difference between the calculation and the data is present - and the } \\
\text { difference is not understood. Such a difference could follow from errors in either } \\
\text { the calculation or the portrayal of the data-or an inadequate code model of the } \\
\text { phenomenon. }\end{array}$ \\
\hline
\end{tabular}

i. Property conservation issues arise when two calculations of the same property are performed by a systems code using two different algorithms or methods. This practice may follow in an effort to enhance the accuracy of the code result. Because the two methods are likely to calculate slightly different values of the same property such as pressure, property conservation must be considered. 


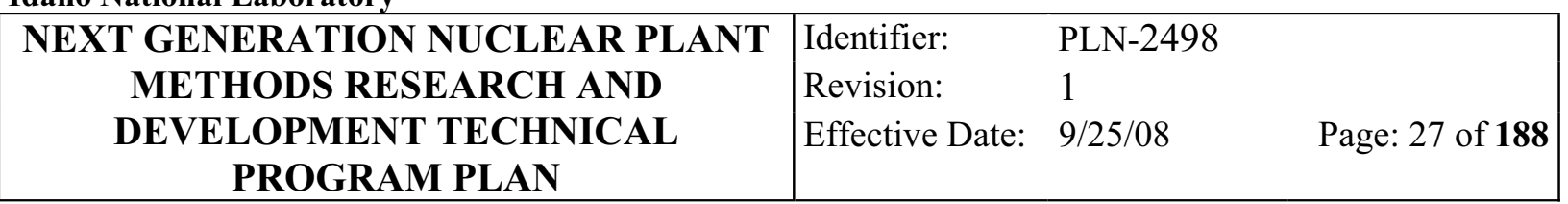

- Scalability. Experimental scaling distortions are identified and isolated, e.g., inappropriate environmental heat losses that stem from the larger surface-to-volume ratios that are inherent to scaled facilities. Finally, an effort to isolate all code scaling distortions is performed through code assessment calculations. Scaling distortions may arise from an inappropriate use of a correlation developed in a small-scale system when applied to a full-scale system.

\subsubsection{Validation Process}

Validation of the analysis tools (e.g., the systems analysis and CFD software) will proceed using a process designed to include the expertise in not only the nuclear industry but the expertise external to the nuclear industry when required. Participation by experts at the national laboratories together with university experts and industry experts will ensure the software tools achieve the defined objectives.

The process is centered on defining a validation matrix that serves as the foundation for a set of standard problems for both systems analysis and CFD software. The validation matrix is assembled by correlating the key phenomena identified in the PIRT for the most challenging scenarios with the available data sets. If data sets are needed but not available, experiments will be designed and performed to provide the needed data. The experiments will be specified to meet the standard required for software validation, that is, with a reasonable uncertainty band and a data range that either includes the required validation range or can be scaled to include the required validation range. Subsequently, the data sets become the basis for standard problems that will be used by the VHTR validation community. The VHTR validation community consists of users in Boxes 2 through 5 in Figure 3: national laboratory users, university community, GIF community (including the vendor community), and the community of users (who may be outside the nuclear community) for the software being validated. This process is shown in Figure 10.

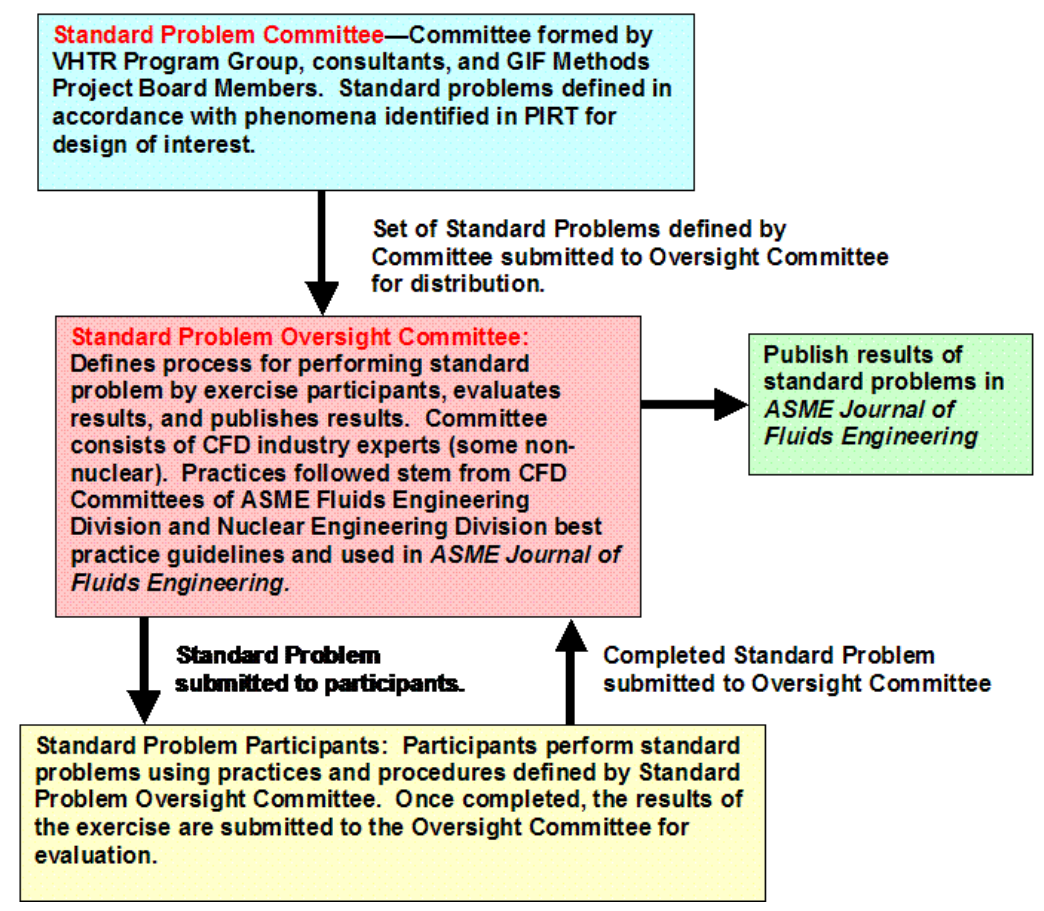

Figure 10. Validation process, including participation by experts from the national laboratories, universities, vendors, and the community specific to the software undergoing validation. 


\begin{tabular}{|c|lll|}
\hline NEXT GENERATION NUCLEAR PLANT & Identifier: & PLN-2498 \\
METHODS RESEARCH AND & Revision: & 1 & \\
DEVELOPMENT TECHNICAL & Effective Date: & $9 / 25 / 08$ & Page: 28 of 188 \\
PROGRAM PLAN & & & \\
\hline
\end{tabular}

The process for specifying standard problems begins with the formation of a "Standard Problem Committee" (presently being formed through the auspices of the GIF Methods Project Management Board). The Standards Problem Committee will consist of members from selected universities, the VHTR Program Group, GIF organizations, and the vendors. The standard problems will be defined to meet a prescribed standard.

The validations performed using standard problems will be assigned to those who will perform validation exercises by the "Standard Problem Oversight Committee" who will also formulate the practices and procedures that will be used for performing the validation calculations. This committee will be composed of experts in the use and validation of the software. For example, one of the committee members for the CFD Standard Problem Oversight Committee will be an expert selected by the ASME CFD Technical Committee, which was previously responsible for the well-known CFD Triathlon in the 90s, who will not necessarily be a member of the nuclear community. Other members will be from the VHTR Program Group, universities, and perhaps the vendors. Following assignment of the standard problems to the participants, the committee will also oversee the final review and publication of the validation studies in the literature as shown in Figure 10 above.

\subsection{Software Tool Selection and Software Development}

\subsubsection{Software tool selection}

When confronted with the need to calculate some of the phenomena that will be encountered in the VHTR scenarios, it is inevitable that analysts will be required to choose one software tool over another. This will be particularly true of systems analysis software (for example, GRSAC, MELCOR, and RELAP5; see Figure 8 above). To assist the analyst in formally choosing software, a methodology is given in Figure 11 where a flow chart summarizes key factors and questions such as:

- Has the software ever been used to analyze the phenomena or scenario? By answering this question the analyst may be introduced to references and other experts who have applied the software to similar phenomena or scenarios. Hence a body of useful information may be available.

- Are the phenomena modeled properly? And does the model region of applicability correspond to the system phenomena or scenario envelope? These questions may be most easily answered by using the required manuals and documentation identified in Figure 9 above, e.g., models and correlations, theory manual, scaling relationships and applications, developmental assessment reports, validations.

- Have validation studies been completed for the phenomena or scenario? Were the validation results reasonable or excellent (as defined in Table 2) — or were the results minimal or unacceptable? If a body of validation results are not available, or if the validation results were not "reasonable" as a minimum, then either the software should not be used or it should be validated to ensure that the calculated results are reliable rather than misleading. 


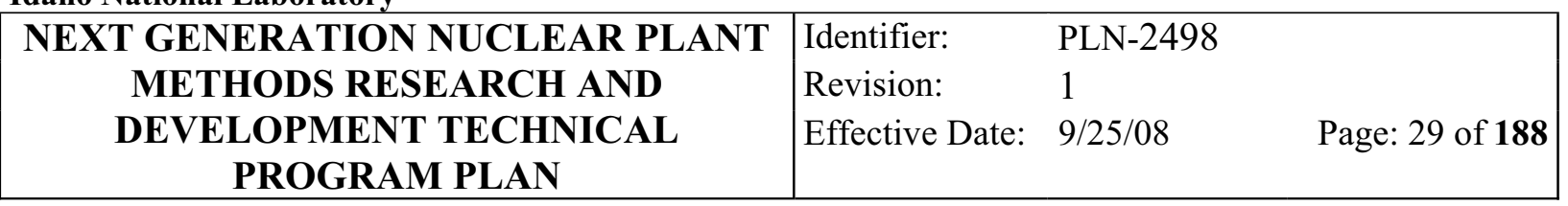

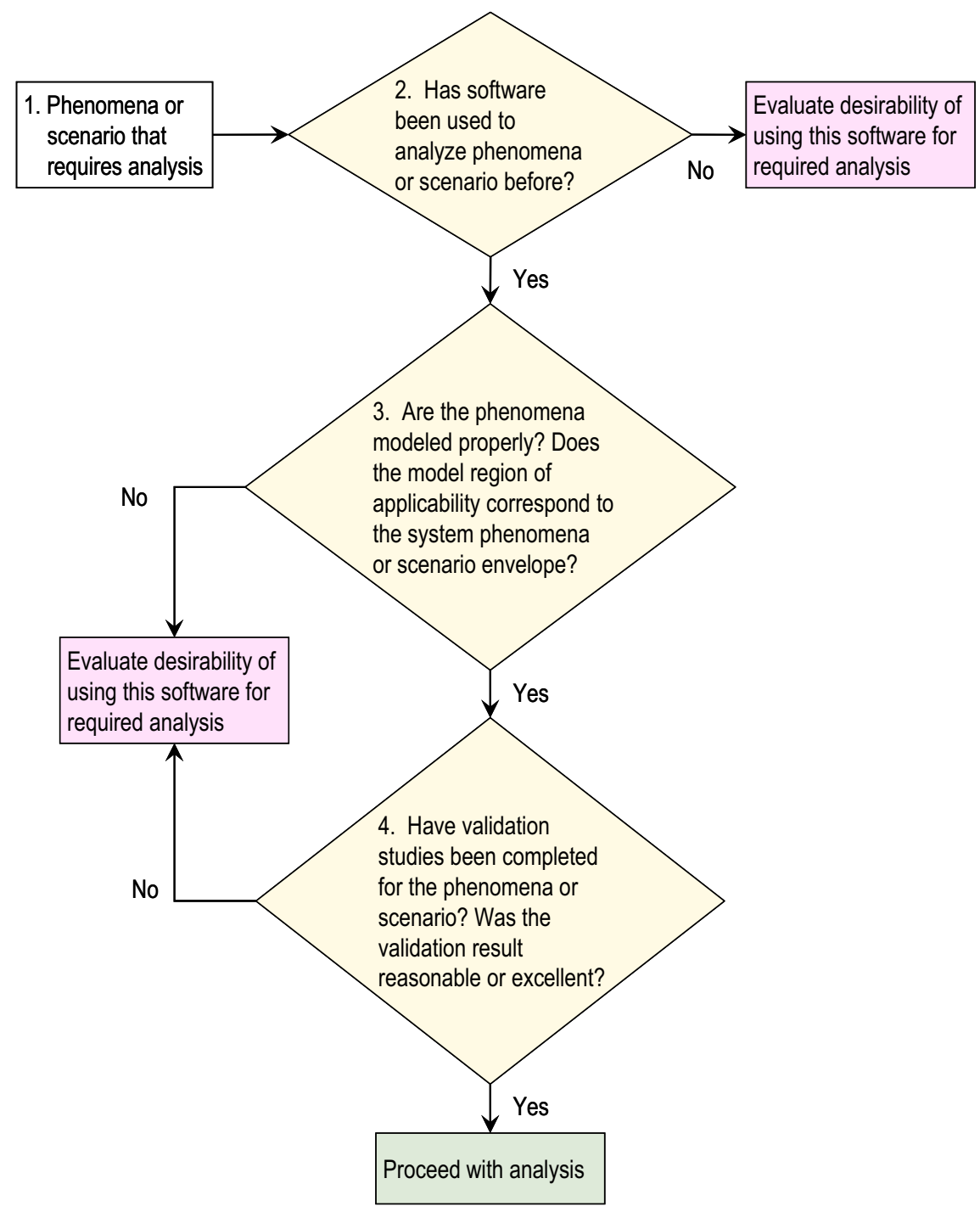

Figure 11. Flow chart to evaluate applicability of analysis software.

Only when acceptable answers are obtained for the questions listed above, can the software under consideration be used for the required analysis with confidence.

\subsection{Analysis of Operational and Accident Scenarios and Expert Review}

Throughout the process outlined in Figure 3 above, when the program achieves key goals or has specific needs for guidance or direction, an expert review will take place. The review may occur in many ways, including a NERAC review, specially convened workshop, etc.

During fiscal year 2006 several reviews of the Design and Safety Methods Validation Program were performed. Program personnel met with staff members of PBMR Pty and Areva Ltd. Finally, a methods workshop was held in Salt Lake City in August 2006 to review the NGNP methods R\&D plans. 


\begin{tabular}{|c|lll|}
\hline NEXT GENERATION NUCLEAR PLANT & Identifier: & PLN-2498 \\
METHODS RESEARCH AND & Revision: & 1 & \\
DEVELOPMENT TECHNICAL & Effective Date: & $9 / 25 / 08$ & Page: 30 of 188 \\
PROGRAM PLAN & & & \\
\hline
\end{tabular}

\subsubsection{Summary of Meetings with PBMR Pty and Areva Ltd}

Design and Safety Methods Validation Program personnel visited PBMR Pty engineering offices in late March and early April 2006 and Areva Ltd engineering offices in July 2006. In both visits program personnel gave extensive presentations reviewing the ongoing $R \& D$ at the national laboratories. Information was interchanged at both meetings. A number of specific potential collaborations were identified for further discussion. These potential collaborative efforts are still under discussion and the notes are available to interested parties.

\subsubsection{Summary of Methods Workshop in Salt Lake City}

The NGNP Design Methods Workshop was held in Salt Lake City, Utah on August 22 and 23, 2006, to discuss the direction being taken in methods R\&D planning. The audience was comprised of technical staff from: Areva Ltd; Westinghouse, Inc, Technology Insights Inc, NRC; Oregon State University, Massachusetts Institute of Technology, University of Idaho, ANL, INL, and Oak Ridge National Laboratory. The experts from these organizations were about equally divided between the reactor physics and thermal fluids areas.

The workshop progressed by first discussing the gaps and then addressing how those gaps might be eliminated or minimized. The gaps refer to whether or not the software tools under consideration are capable of calculating the phenomenon of interest. If the software isn't capable of calculating the required phenomenon, the discussion centered on what sort of planning is ongoing to ensure the software tools under consideration are either replaced by software that can perform the necessary calculations or are being validated adequately. Following a set of presentations designed to acquaint the workshop participants with NGNP methods R\&D planning to date, the workshop was divided into two groups that addressed reactor physics and thermal-fluids behavior, respectively.

The tools discussed under the heading of reactor physics fall into two groups: tools for the calculation of reactor physics parameters and tools for the analysis of pebble flow mechanics. The first group includes tools for both pebble-bed and prismatic reactors, the second group only includes tools applicable to the pebble-bed. The parameters to be calculated by each group of tools are listed below:

The Group I tools calculate reactor physics parameters, including: multiplication factor, reactivity worths and swing, reactivity coefficients, flux distribution, fluence distribution, power distribution, point kinetics parameters, fuel burnup distribution, and nuclide density distribution. The Group II tools address pebble flow mechanics and describe pebble flow patterns.

For each of the parameters listed above, comments were recorded for each of the following categories of tools (The outcome of the discussions on these tools and parameters are given in Appendix B.):

- ENDF data processing tools

- Stochastic capabilities

- Lattice-cell tools (for preparation of multi-group cross sections)

- Whole-core analysis tools (multi-step design approach)

- Whole-core analysis tools (spatially heterogeneous modeling)

- Source term/decay heat determination

- Coupled neutron-gamma calculation 


\begin{tabular}{|c|lll|}
\hline NEXT GENERATION NUCLEAR PLANT & Identifier: & PLN-2498 \\
METHODS RESEARCH AND & Revision: & 1 & \\
DEVELOPMENT TECHNICAL & Effective Date: & $9 / 25 / 08$ & Page: 31 of 188 \\
PROGRAM PLAN & & & \\
\hline
\end{tabular}

- Spatial kinetics calculation

- Integral data for validation.

The tools discussed under the heading of thermal-fluids behavior fall within three groups: computational fluid dynamics, systems analysis, and severe accident software. Of these software types, CFD software can be used to analyze the fluid dynamics in any portion of the NGNP. The specific strength of CFD is its capability to analyze the presence and location of localized hot spots and thermal gradients. The largest impediment to using CFD is the computational requirements and the size of the problem that requires analysis. Systems analysis software (such as RELAP5) can also be used to analyze the fluid dynamics in any portion of the NGNP, but only 1-D analyses can be performed with highfidelity. GRSAC is a very fast-running code that is ideal for performing scoping calculations that describe not only core temperature distributions and other important temperature distributions (e.g., vessel temperatures and confinement material temperatures) as a function of time, but also graphite oxidation calculations due to air ingress. MELCOR is also a fast-running code that will be used to calculation fission product transport from the vessel into the reactor confinement.

Presently, all the gaps, issues, and inadequacies identified by workshop participants are being addressed either directly by formulating the practices and procedures that are to be used for planned V\&V or are planned to be implemented at some stage by the Design and Safety Methods Validation Program. Further details are given in Appendix B.

The final stage in the Methods R\&D process is the performance of the required analyses and calculations to meet the needs identified by DOE and the VHTR program. Presently, these needs have not been defined, except from an overall perspective.

\subsubsection{Summary}

The practices, procedures, and methodologies described in this section provide the mechanism for the Methods R\&D Program to achieve its required objectives. Although some software development has been identified and is ongoing, it is clear that the great bulk of the work resides in verifying and validating the array of software tools required to analyze the behavior of the NGNP at operational, off-normal, and accident conditions. A spectrum of physical experiments is required to enable proper validation of the applicable software. The distribution between the work required in the reactor physics area and the thermal-fluids area is estimated to be approximately $16 \%$ and $84 \%$, respectively. 


\begin{tabular}{|c|lll|}
\hline NEXT GENERATION NUCLEAR PLANT & Identifier: & PLN-2498 & \\
METHODS RESEARCH AND & Revision: & 1 & \\
DEVELOPMENT TECHNICAL & Effective Date: & $9 / 25 / 08$ & Page: 32 of 188 \\
PROGRAM PLAN & & & \\
\hline
\end{tabular}

\section{NUCLEAR DATA: INTEGRAL EXPERIMENT EVALUATIONS, SENSITIVITY STUDIES, AND DIFFERENTIAL CROSS SECTION MEASUREMENTS}

Integral benchmark experiment data for existing critical configurations that are neutronically similar to contemplated VHTR designs are required for physics code validation and quality assurance, both as part of the reactor design process and for licensing applications. Modern computational simulation techniques for reactor physics are capable of very high accuracy, and can, in some cases, replace significantly more costly mockups and critical experiments, but only if the accuracy of the simulation is carefully established by rigorous validation of physics codes against appropriate integral experiment physics data. In addition, mathematically rigorous sensitivity studies for representative VHTR core designs are required as an aid in guiding the design of any needed critical experiments that cannot be replaced by simulations (because experiments with sufficient similarity are determined to be unavailable), and perhaps most importantly, for quantification of the propagation of uncertainties in computational simulations due to uncertainties in the underlying nuclear data and other parameters that make up the input to the simulation models.

Finally, high-accuracy differential nuclear data (nuclear cross section) libraries are required as input for all computational reactor physics tasks associated with VHTR design, licensing, and subsequent operation. Any simulation is only as accurate as the input data, and in the reactor physics field, the differential nuclear cross sections for the various materials used in the reactor constitute the most fundamental and crucial input information needed for the computational simulation process. For example, computational studies performed at INL show that for a reference prismatic VHTR fuel design, an uncertainty of as little as $10 \%$ in the ${ }^{240} \mathrm{Pu}$ capture cross-section can lead to uncertainties in system reactivity of as much as $500 \mathrm{pcm}$ absolute reactivity because of the propagated uncertainty in ${ }^{241} \mathrm{Pu}$ buildup. This is an indication of high sensitivity to this particular cross-section. Furthermore, earlier integral-experiment-based code validation studies performed and published by INL (Sterbentz 2002 and Sterbentz and Wemple 1996) for low-enriched fuel with thermal or slightly hyperthermal neutron spectra representative of typical VHTR designs show that computations of the inventories of the plutonium isotopes of interest can vary by as much as $30 \%$ from corresponding measurements at burnups of less than one-third of what is contemplated in a baseline VHTR scenario. Such discrepancies can propagate in a way that has major effects on the uncertainty of computed safety-related reactor parameters such as reactivity and Doppler feedback.

The following three sections describe the near-term key VHTR-related needs, planned activities, and key milestones in these three areas.

\subsection{Integral Neutronic Parameter Evaluations for V\&V and Assessment of Needs for New Measurements}

Design and safety analysis calculations for the VHTR will require calculation of k-effective, neutron flux distribution, and reaction rates and cross-sections, along with quantities that can be derived from flux and cross-sections such as depletion and power distribution. To confirm that analysis codes can predict these quantities with sufficient accuracy, the codes must be benchmarked against experimental measurements made in the closest possible conditions to those expected in the VHTR. Conditions relevant to benchmarking VHTR codes include geometry, fuel type, and, for a pebble-bed-type experimental facility, whether it achieved an asymptotic state. Code-calculated quantities to be compared with experimental data include k-effective, flux distributions (where measured values are available), and 


\begin{tabular}{|c|lll|}
\hline NEXT GENERATION NUCLEAR PLANT & Identifier: & PLN-2498 \\
METHODS RESEARCH AND & Revision: & 1 & \\
DEVELOPMENT TECHNICAL & Effective Date: & $9 / 25 / 08$ & Page: 33 of 188 \\
PROGRAM PLAN & & & \\
\hline
\end{tabular}

spectral indices (key reaction rate ratios used to determine whether the neutron energy spectra are comparable). Finally, a key concern in selecting appropriate benchmarks is related to whether the required data are readily available from an experimental facility.

\subsubsection{Integral Experiment Assessment for VHTR Methods Validation Applications}

Code packages are typically verified and validated. Verification is the assurance that the code actually performs as designed, that is, it accurately solves the equations that represent the physical phenomena occurring in the systems. Verification is typically accomplished using models, software inspections, and by comparing code solutions to analytical solutions for simple problems, or to solutions from previously verified and validated codes, or to solutions from higher fidelity codes. Validation gives the assurance that the models employed can provide physically valid representations of the system characteristics and can be used to derive core parameters. Validation is usually accomplished by comparing calculated results to experimental measurements or results of other computational models that have already been validated against relevant measurements.

In FY 2004, ANL and INL examined information on several past and present experimental and prototypical facilities based on High Temperature Gas Reactor (HTGR) concepts that could potentially be used as the V\&V basis of codes employed in the design and analysis of the VHTR cores. A preliminary assessment of the applicability of the existing test data for benchmarking the pebble-bed and prismatic block-type cores was performed as part of that effort (Terry et al. 2004). The experiments assessed included.

- Pebble-bed type cores: ASTRA, AVR, CESAR II, GROG, HTR-10, HTR-PROTEUS, KAHTER, SAR, and THTR

- Prismatic block-type cores: CNPS, DRAGON, FSV, GGA HTGR Criticals, HITREX-1, HTLTR, High-Temperature Test Reactor (HTTR), MARIUS-IV, Peach Bottom HTGR, Peach Bottom Criticals, SHE, NESTOR/HECTOR, and VHTRC.

Tables 3 and 4 compare the facilities discussed above with respect to various qualities desired for use in benchmarking computer codes. One of the column headings has different meanings in the two tables. A PBR operating at constant power for a sufficiently long time (on the order of 2 or 3 years) will approach asymptotic distributions of neutron flux and compositions. In principle, except to replace radiationdamaged reflector components, the PBR never needs to be shut down, so these asymptotic distributions will be approached more and more closely as time goes on. Prismatic-type reactors are batch-loaded so the compositions change continuously with time. They do not approach asymptotic distributions as PBRs do, and operation is interrupted at intervals of roughly two years for fuel removal, shuffling, and replacement. However, after several operating cycles, the distribution of compositions at cycle startup approaches an asymptotic configuration. The column heading "Asymptotic State or Zero-Power Startup" refers to the true time-independent asymptotic configuration for PBRs, but to the cycle-independent startup configuration for prismatic-type reactors.

Trends were observed in the experiments that were performed in the various facilities investigated. It was found that most of the experiments for block-type cores were performed in the United States, while those on pebble-bed cores were done predominantly in Europe. Most of the early U.S. experiments used highly enriched uranium. This was not typically the case for the European experiments. Experiments are currently being performed for both pebble-bed and block type cores in Asia (Japan and China) as well as in Russia. Under this VHTR program element, we will have the opportunity to influence the direction of these experiments in a way that enhances the specific benefit to the VHTR effort. 
Idaho National Laboratory

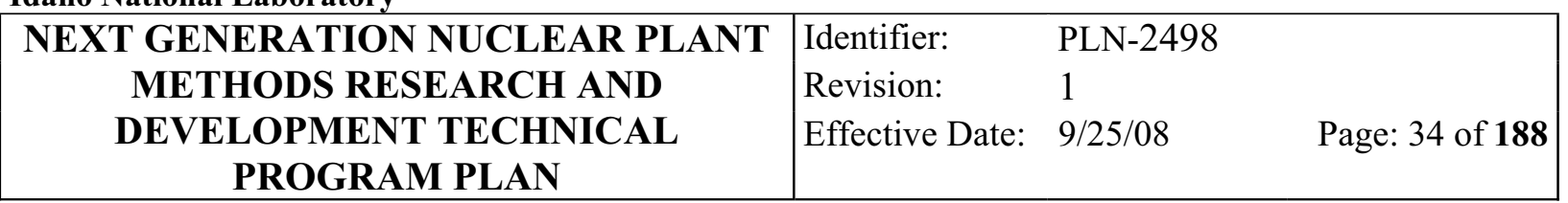

Table 3. Comparison of facilities for Pebble-Bed type cores for benchmarking models.

\begin{tabular}{|c|c|c|c|c|c|c|}
\hline $\begin{array}{l}\text { Facility } \\
\text { (Country) }\end{array}$ & Geometry & Size & Fuel type & $\begin{array}{l}\text { Asymptotic state } \\
\text { or zero-power } \\
\text { startup }\end{array}$ & $\begin{array}{c}\text { Availability of } \\
\text { data }\end{array}$ & Priority \\
\hline $\begin{array}{l}\text { ASTRA } \\
\text { (Russia) }\end{array}$ & $\begin{array}{l}\text { Annular, but } \\
\text { not } \\
\text { azimuthally } \\
\text { symmetric } \\
\end{array}$ & Small & As desired & $\begin{array}{l}\text { Zero-power } \\
\text { startup }\end{array}$ & $\begin{array}{l}\text { Existing facility; } \\
\text { data can be } \\
\text { obtained }\end{array}$ & High \\
\hline $\begin{array}{l}\text { AVR } \\
\text { (Germany) }\end{array}$ & Cylindrical & $\begin{array}{l}\text { Short; radial } \\
\text { extent } \\
\text { appropriate }\end{array}$ & $\begin{array}{l}\text { Various; some } \\
\text { low-enrichment } \\
\text { TRISO } \\
\end{array}$ & Both & Limited & High \\
\hline $\begin{array}{l}\text { CESAR II } \\
\text { (France) }\end{array}$ & Hexagonal & Small & $\begin{array}{l}\text { Low-enriched } \\
\mathrm{UO}_{2}\end{array}$ & $\begin{array}{l}\text { Zero-power } \\
\text { startup }\end{array}$ & $\begin{array}{l}\text { Neutronics data } \\
\text { exist }\end{array}$ & Medium \\
\hline $\begin{array}{l}\text { GROG } \\
\text { (Russia) }\end{array}$ & $\begin{array}{l}\text { Cylindrical } \\
\text { or annular }\end{array}$ & $\begin{array}{l}\text { Short; radial } \\
\text { extent } \\
\text { appropriate }\end{array}$ & $\begin{array}{l}\text { As desired, but } \\
\text { very low packing } \\
\text { fraction }\end{array}$ & $\begin{array}{l}\text { Zero-power } \\
\text { startup }\end{array}$ & $\begin{array}{l}\text { Existing facility; } \\
\text { data can be } \\
\text { obtained }\end{array}$ & Medium \\
\hline $\begin{array}{l}\text { HTR-10 } \\
\text { (China) }\end{array}$ & Cylindrical & Small & $\begin{array}{l}\text { Low-enriched } \\
\text { TRISO }\end{array}$ & Both & $\begin{array}{l}\text { Existing facility; } \\
\text { data can be } \\
\text { obtained }\end{array}$ & Highest \\
\hline $\begin{array}{l}\text { HTR- } \\
\text { PROTEUS } \\
\text { (Switz.) } \\
\end{array}$ & Cylindrical & Small & $\begin{array}{l}\text { LEU pebble-bed } \\
\text { fuel }\end{array}$ & Zero-power & $\begin{array}{l}\text { PSI and IAEA } \\
\text { would need to be } \\
\text { contacted }\end{array}$ & High \\
\hline $\begin{array}{l}\text { KAHTER } \\
\text { (Germany) }\end{array}$ & Cylindrical & Small & Uncertain & $\begin{array}{l}\text { Zero-power } \\
\text { startup }\end{array}$ & Uncertain & High \\
\hline $\begin{array}{l}\text { SAR } \\
\text { (Austria) }\end{array}$ & Cylindrical & Small & $\begin{array}{l}\text { Probably low- } \\
\text { enrichment } \\
\text { TRISO }\end{array}$ & $\begin{array}{l}\text { Zero-power } \\
\text { startup }\end{array}$ & $\begin{array}{l}\text { Limited data were } \\
\text { obtained for this } \\
\text { special-purpose } \\
\text { test }\end{array}$ & Low \\
\hline $\begin{array}{l}\text { THTR } \\
\text { (Germany) }\end{array}$ & Cylindrical & Large & $\begin{array}{l}\text { Thorium- } \\
\text { uranium }\end{array}$ & $\begin{array}{l}\text { Most data for zero } \\
\text { power; reactor } \\
\text { presumably } \\
\text { achieved steady } \\
\text { state }\end{array}$ & $\begin{array}{l}\text { More data } \\
\text { available for zero- } \\
\text { power startup than } \\
\text { operating } \\
\text { conditions }\end{array}$ & Medium \\
\hline
\end{tabular}




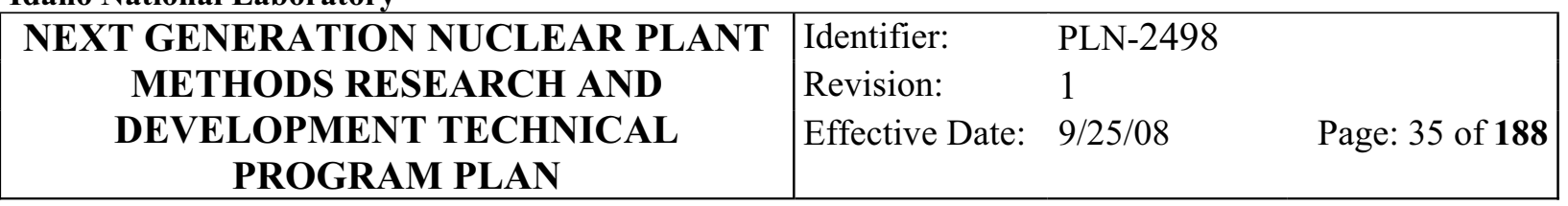

Table 4. Comparison of Facilities for Prismatic Block-Type cores for Benchmarking Models.

\begin{tabular}{|c|c|c|c|c|c|c|}
\hline $\begin{array}{l}\text { Facility } \\
\text { (Country) }\end{array}$ & Geometry & Size & Fuel type & $\begin{array}{l}\text { Asymptotic } \\
\text { state or zero- } \\
\text { power startup }\end{array}$ & $\begin{array}{c}\text { Availability of } \\
\text { data }\end{array}$ & Priority \\
\hline $\begin{array}{l}\text { CNPS } \\
\text { (USA) }\end{array}$ & Cylindrical & Small & LEU & Zero & LANL data & High \\
\hline $\begin{array}{l}\text { DRAGON } \\
\text { (England) }\end{array}$ & Hexagonal & Small & $\mathrm{HEU} / \mathrm{Th}$ & Both & $\begin{array}{l}\text { Data must be } \\
\text { retrieved from } \\
\text { U.K./OECD }\end{array}$ & Low \\
\hline $\begin{array}{l}\text { Fort St. Vrain } \\
\text { (USA) }\end{array}$ & Cylindrical & Large & HEU/Th & Both & $\begin{array}{l}\text { Data is GA } \\
\text { proprietary }\end{array}$ & Medium/High \\
\hline $\begin{array}{l}\text { GGA HTGR criticals } \\
\text { (USA) }\end{array}$ & Cylindrical & Small & HEU & Zero & $\begin{array}{l}\text { Data is GA } \\
\text { proprietary }\end{array}$ & Medium/High \\
\hline $\begin{array}{l}\text { HITREX-1 } \\
\text { (USA) }\end{array}$ & Hexagonal & Small & LEU fuel & Zero & $\begin{array}{l}\text { U.K. nuclear } \\
\text { data }\end{array}$ & Medium/High \\
\hline $\begin{array}{l}\text { HTLTR } \\
\text { (USA) }\end{array}$ & Block & Small & $\mathrm{Pu}-\mathrm{Th}$ fuel & Zero & PNNL data & Low \\
\hline $\begin{array}{l}\text { HTTR } \\
\text { (Japan) }\end{array}$ & $\begin{array}{l}\text { Cylindrical/ } \\
\text { Annular }\end{array}$ & Small & LEU fuel & Both & $\begin{array}{l}\text { Existing } \\
\text { facility; data } \\
\text { can be obtained }\end{array}$ & High \\
\hline $\begin{array}{l}\text { MARIUS-IV } \\
\text { (France) }\end{array}$ & Unknown & Small & HEU-Th & Zero & Unknown & Low \\
\hline $\begin{array}{l}\text { Peach Bottom HTGR } \\
\text { (USA) }\end{array}$ & Cylindrical & Small & HEU/Th & Both & $\begin{array}{l}\text { Data is GA } \\
\text { proprietary }\end{array}$ & Low \\
\hline $\begin{array}{l}\text { Peach Bottom } \\
\text { Criticals } \\
\text { (USA) }\end{array}$ & Cylindrical & Small & LEU/Th & Zero & $\begin{array}{l}\text { Data is GA } \\
\text { proprietary }\end{array}$ & Low \\
\hline $\begin{array}{l}\text { SHE } \\
\text { (Japan) }\end{array}$ & Hexagonal & Small & LEU fuel & Zero & JAEA data & Medium/High \\
\hline $\begin{array}{l}\text { NESTOR/HECTOR } \\
\text { (England) }\end{array}$ & $\begin{array}{l}\text { Square and } \\
\text { cylindrical }\end{array}$ & Small & LEU fuel & $\begin{array}{l}\text { Zero and } \\
\text { elevated } \\
\text { temperatures }\end{array}$ & $\begin{array}{l}\text { U. K. nuclear } \\
\text { data }\end{array}$ & Medium/High \\
\hline $\begin{array}{l}\text { VHTRC } \\
\text { (Japan) }\end{array}$ & Hexagonal & Small & LEU fuel & Zero & JAEA data & High \\
\hline
\end{tabular}

HTGR core designs have evolved to improve system economy and safety. The current basic VHTR concepts of interest include both the prismatic (fixed-fuel) design as well as the pebble-bed (moving fuel, continuous online refueling) design. Both concepts have many different technical aspects compared to those of the early HTGRs. These differences limit the applicability and usefulness of the existing experimental data to VHTR core designs. Additionally, in the case of the data produced on national or commercial bases, the availability of those data might be quite limited.

The 2004 assessment revealed that the VHTR systems under development in the Generation IV program differ in significant ways from previous high-temperature reactors (thorium utilization, highly 


\begin{tabular}{|c|lll|} 
NEXT GENERATION NUCLEAR PLANT & Identifier: & PLN-2498 \\
METHODS RESEARCH AND & Revision: & 1 & \\
DEVELOPMENT TECHNICAL & Effective Date: & $9 / 25 / 08$ & Page: 36 of 188 \\
PROGRAM PLAN & & & \\
\hline
\end{tabular}

enriched fuel, BISO versus TRISO fuel, thermal efficiency, operating temperatures, etc). These differences limit the applicability and direct usefulness of some of the existing experimental data for NGNP core designs. It was acknowledged that the availability of the data produced on a commercial basis or by foreign governments might be limited. An effort was made to identify experimental tests of the highest priority, recover the data for those cases, and then develop standard problems (benchmarks) that are of sufficient quality for use in the licensing of the VHTR analysis codes. A set of criteria employed to judge the relevance of the different tests included: purpose of the previous experiment, geometry of core, fuel forms, core materials, physics parameters measured, measurement state, availability of design and uncertainty data, and applicability of data to $\mathrm{V} \& \mathrm{~V}$. Based on these criteria, the experiments judged to be of the highest priorities are:

- $\quad$ ASTRA, AVR, High Temperature Gas-Cooled Reactor-10 (HTR-10), HTR-PROTEUS for the pebble-bed cores

- HTTR, VHTRC, and CNPS for the prismatic block-type cores.

Further information resulting from this assessment and some follow-up studies is summarized below.

The AVR core was used for many experiments pertinent to the development of high temperature reactors (HTRs). During its lifetime, the core was loaded with a variety of fuel pebbles of different types and enrichments, which were generally mixed in the approach to equilibrium loading for a given fuel type. Consequently, there was never a clean core that would provide the well-characterized, quality data required for benchmarking reactor physics codes. This is explained by the fact that the AVR was a power reactor (46 MWt) built for integrated demonstration of HTR concepts; nonetheless, this experiment remains of high importance precisely because some data for at-power conditions may be available.

The ASTRA, HTR-PROTEUS, and HTR-10 test data would, however, be directly useful to the validation of tools being developed for the analysis of the pebble-bed VHTRs. These experiments include those with an inner reflector similarly to the design of current VHTRs. The attraction of ASTRA and HTR-10 is that they are facilities in which additional experiments could be performed, if necessary, under international collaborations. The HTR-PROTEUS experiments performed in the 1990s offer very clean data for evaluating the performance of codes for VHTR analysis. Collectively, it appears that the data offered by these facilities would be adequate for the V\&V needs of tools for pebble-bed cores.

Preliminary evaluations of the existing physics experimental data for these cores (particularly ASTRA and HTR-10) indicate that the pertinent uncertainty data for specifying detailed benchmarks might be inadequate. In that case, options would include (1) obtaining the pertinent data from the institutions that originally performed the experiments, (2) arranging for additional well-characterized experiments to be performed in the ASTRA and HTR-10 facilities, or (3) reconfiguring the PROTEUS core as a pebble-bed core and performing experiments for VHTR designs; PROTEUS is currently configured for LWR experiments. The approach of reconfiguring cores could also be considered for one of the existing national laboratory or university reactors in the United States.

Startup test data for the FSV reactor core would appear to be directly useful for the validation of reactor physics tools for VHTR cores. It has, however, been found that there are differences in core designs that result in deficiencies in the data required for V\&V of tools for prismatic VHTRs: the FSV core used thorium-uranium fuels, while low enriched uranium fuels are being considered for the NGNP; the FSV core did not use an annular core design typical of all the VHTR cores being considered for the NGNP; the annular core has been found necessary to meet the passive safety design goal of the NGNP; and the arrangement of control rods in the two cores is different. The prismatic VHTR design employs 


\begin{tabular}{|c|lll|}
\hline NEXT GENERATION NUCLEAR PLANT & Identifier: & PLN-2498 \\
METHODS RESEARCH AND & Revision: & 1 & \\
DEVELOPMENT TECHNICAL & Effective Date: & $9 / 25 / 08$ & Page: 37 of 188 \\
PROGRAM PLAN & & & \\
\hline
\end{tabular}

separate safety and regulating rods. The regulating rods are deployed asymmetrically in the radial reflector blocks in these designs, which make determining their reactivity worths quite difficult for deterministic neutronics codes; safety rods are also placed asymmetrically in fuel blocks. While stochastic Monte Carlo tools could be used as reference solutions against which the deterministic tools (typically used for routine calculations) could be qualified, there would still be a need to validate both types of codes to allow their use in the design and safety analyses of VHTR cores. Thus, the FSV data are insufficient for qualifying physics tools for VHTR designs.

The most pertinent data for prismatic VHTR analyses are the experiment test data derived from the VHTRC and High Temperature Engineering Test Reactor (HTTR) experiments. The two facilities are based at the Japan Atomic Energy Agency (JAEA, formerly JAERI) in Japan. The VHTRC critical experiments were used to support the development of the HTTR experimental power reactor (30 MWt). Preliminary evaluations of the HTTR and VHTRC data using Monte Carlo and deterministic codes show differences between measured and calculated core criticality values (greater than $1 \%$ in $\mathrm{k}_{\text {eff). These }}$ differences can only be resolved by the availability of all pertinent experimental data. Access to the experimental data would have to be negotiated under the GIF. The usefulness of the VHTRC and HTTR data for confirming prediction of power peaking at the core reflector interfaces in prismatic VHTR designs is unclear. The initial criticality of the HTTR was obtained with an annular core. The active core thickness and core dimensions of this core are however not representative of the actual prismatic VHTR designs. The JAEA plans to conduct experiments with annular cores at the HTTR facility in the late 2000s. The NGNP program could participate in the planning and performance of these experiments. Finally, an initial evaluation of the CNPS experiments at Los Alamos (Taiwo et al. 2005) indicates that, although it was difficult to quantify the uncertainty in the experimental core integral parameters arising from data uncertainties, high-fidelity models of the evaluated configurations could be useful as numerical benchmarks.

If additional data that could not be fulfilled by the HTTR experiments are required under the NGNP programs, three primary options remain: consider participation in other international prismatic VHTR experiments, upgrade an old graphite-moderated experimental facility in the United States, or convert an existing national laboratory or university reactor facility into one that could be used for prismatic VHTR physics experiments.

Under an ongoing International Nuclear Energy Research Initiative (I-NERI) Project (Project 2004005 F, "Assessment of Existing Physics Experiments Relevant to VHTR Designs," T. Taiwo [ANL] and R. Jaqmin [CEA]) involving CEA, ANL, and INL, the CEA has evaluated the need for additional physics experiments for VHTR cores. The preliminary evaluation indicates that new, reliable, high-quality experimental data will be needed for validation purposes, unless core designers are willing to accept very large uncertainties, which might be insufficient in meeting the requirements of the licensing authorities. CEA is also investigating the feasibility of new experiments in its facilities. If it is determined that such an experimental physics program is feasible in one of the CEA facilities, it has been indicated to the United States that the experiment would be open to international participation. Access could be gained by contributing material and effort. This approach might be an option to the U.S. NGNP project. Even in that case, it is desirable that the U.S. national laboratories be actively involved in the planning and performance of the experiments. 


\begin{tabular}{|c|lll|} 
NEXT GENERATION NUCLEAR PLANT & Identifier: & PLN-2498 \\
METHODS RESEARCH AND & Revision: & 1 & \\
DEVELOPMENT TECHNICAL & Effective Date: & $9 / 25 / 08$ & Page: 38 of 188 \\
PROGRAM PLAN & & & \\
\hline
\end{tabular}

The following activities must be conducted to determine the need for additional experiments and obtain the necessary data:

- Identify and assess experimental and operational data and numerical benchmarks relevant to licensing of core physics analysis code system; compile and analyze selected experimental and numerical benchmarks.

- Identify needs for additional experiments for code $V \& V$ and where to conduct the experiments (locally or internationally).

- Participate in the planning, conduct, and pre- and post-analysis of experiments that would support the licensing of the annular designs being considered for the passively-safe VHTRs; measurements of core criticality (critical mass), power/reaction rate distributions, reactivity worths/defects, and kinetic parameters could be part of this effort. The planning study should include justifying such experiments and demonstrating that the experiments represent VHTR designs.

- Conduct and analyze pertinent experiments.

- Use analysis results to identify deficiencies in codes and upgrade the code capabilities.

- Participate in international efforts directed toward assessing, validating, and qualifying codes and data for VHTR/NGNP analysis.

\subsubsection{Protocols for Evaluating Integral Nuclear Data for Quality Assurance Purposes}

In the general context outlined above, the near-term steps under this R\&D plan involve detailed evaluation and documentation of the most promising selected high-priority benchmarks to provide benchmark specifications that are accepted by the community and by regulators for validation of physics modeling codes. The work can be conducted under the auspices of the International Reactor Physics Evaluation Project (IRPhEP), an international effort that was established by the Organization of Economic Cooperation and Development (OECD) Nuclear Energy Agency (NEA) Nuclear Science Committee (NSC) in June of 2003. Integral nuclear data submitted for evaluation under the protocols of the IRPhEP is published in an OECD Handbook available to all participating countries. Because of the rigorous quality standards involved in the evaluation process, published IRPhEP benchmarks will have the highest reasonably achievable level of international credibility and a-priori regulatory acceptance, as discussed in the following paragraph. In FY 2005 and FY 2006, five of the high-priority cases (HTR-10, HTR-PROTEUS, CNPS, HTTR, and VHTRC) were, in fact, further evaluated for purposes of better understanding and refining their utility, as noted earlier, and in FY 2006 the HTR-10 Evaluation became the first of these to be formally accepted for inclusion in the IRPhEP Handbook (OECD/NEA 2006)

Protocols for the IRPhEP are patterned after those of the closely-related OECD-NEA International Criticality Safety Benchmark Evaluation Project (ICSBEP) for evaluation and preservation of integral data suitable for validation of physics codes and data used for ex-core nuclear criticality safety assurance. Over the past 15 years, ICSBEP has become the accepted international mechanism for standardized, peerreviewed archival of this type of quality assurance data. Nuclear criticality safety physics computations, which are benchmarked against integral data that has undergone the ICSBEP certification process, are accepted by the NRC. The IRPhEP seeks to provide the same framework for quality assurance of in-core reactor physics computations, ultimately streamlining this part of the licensing process and reducing associated costs.

IRPhEP evaluations and protocols are closely coordinated with the ICSBEP in order to avoid duplication of effort (both projects are managed through INL for the OECD-NEA NSC). Some 


\begin{tabular}{|c|lll|}
\hline NEXT GENERATION NUCLEAR PLANT & Identifier: & PLN-2498 \\
METHODS RESEARCH AND & Revision: & 1 & \\
DEVELOPMENT TECHNICAL & Effective Date: & $9 / 25 / 08$ & Page: 39 of 188 \\
PROGRAM PLAN & & & \\
\hline
\end{tabular}

benchmark data are applicable to both nuclear criticality safety and reactor physics technology. Some have already been evaluated and published by the ICSBEP; however, ICSBEP efforts are focused primarily on nonreactor critical configurations and have, in general, only mentioned in passing other types of measurements relevant to reactor cores such as reactivity measurements, flux distributions, spectral indices, reaction rates, and $\beta_{\text {eff. }}$ Experiments relevant to reactor physics applications that have already been evaluated and published by the ICSBEP will simply be extended by the IRPhEP to include evaluation and documentation of other reactor physics measurements that were made in conjunction with the assembly of a critical configuration. Measurements that have not been evaluated by the ICSBEP, such as the VHTR-relevant experiments discussed in this technical program plan, will be fully evaluated and documented in accordance with similar guidelines, requirements, and quality assurance measures as they apply to the OECD-ICSBEP.

INL provides leadership for the IRPhEP Technical Review Group organized in FY 2004 and 2005, maintains the infrastructure of the IRPhEP and is responsible for compiling and distributing annual publications. Based on experience with the ICSBEP, DOE will realize significant benefits. Through this effort the IRPhEP will be able to (1) consolidate and preserve the information base that already exists worldwide, (2) retrieve lost data, (3) identify areas where more data are needed, (4) draw upon the resources of the international reactor physics community to help fill those needs, (5) identify discrepancies between calculations and experiments caused by deficiencies in cross-section data, crosssection processing codes and neutronics codes, (6) eliminate a large portion of the tedious and redundant research and processing of reactor physics experiment data, and (7) improve experimental planning, execution, and reporting.

The formal benchmark evaluation process is quite rigorous and includes the following basic steps performed for a given benchmark by the evaluating organization:

Verify the data, to the extent possible, by reviewing original and subsequently revised documentation, and by talking with the experimenters or individuals familiar with the experimenters or the facility

1. Develop analytical models for the specific core configuration measured using standard computer codes and, in the case of this R\&D plan specific to the VHTR, using the codes specifically intended for VHTR analysis

2. Perform appropriate computations and compare the results with the associated experimental data

3. Compile the data and the methodologies (experimental and analytical) into a standardized format

4. Evaluate the data and uncertainties associated with the data

5. Formally document the work into a single source of verified reactor physics measurements data.

Each draft experiment evaluation then undergoes thorough internal review by the evaluator's organization. Reviewers verify the:

- Accuracy of the descriptive information given in the evaluation by comparison with original documentation (published and unpublished)

- Benchmark specification can be derived from the descriptive information given in the evaluation

- Completeness of the benchmark specification

- Results, conclusions, and adherence to format. 


\begin{tabular}{|c|lll|}
\hline NEXT GENERATION NUCLEAR PLANT & Identifier: & PLN-2498 \\
METHODS RESEARCH AND & Revision: & 1 & \\
DEVELOPMENT TECHNICAL & Effective Date: & $9 / 25 / 08$ & Page: 40 of 188 \\
PROGRAM PLAN & & & \\
\hline
\end{tabular}

In addition, each experiment undergoes independent peer review by another IRPhEP Technical Review Group member at a different facility. Starting with the evaluator's submittal in the appropriate format, independent peer reviewers verify:

- That the benchmark specification can be derived from the descriptive information given in the evaluation

- The completeness of the benchmark specification

- The results and conclusions

- Adherence to format.

A third review by the assembled IRPhEP Technical Review Group then verifies that the benchmark specification and conclusions are adequately supported.

Finally, it may be noted that, in addition to careful evaluation of available data, the operating range of the VHTR (temperature and burnup) may well dictate a need for an additional experimental component to validate the reactor physics calculations, including the Monte Carlo ones. The cost of this effort will depend on the type of facility needed for such experiments, but would likely require significant funding not outlined here. As a first approach, attempts would be made to leverage international activities such as HTTR, HTR-10, ASTRA data, and planned activities by U.S. partners in the Gen IV International Forum. If this is insufficient for code validation, expert groups consisting of analysts and experimentalists would be convened to provide justifications for the experiments and estimates of the associated costs. Where additional experimental measurements are required, attempts will be made to make use of existing facilities worldwide. Conclusions will be made concerning adequacy of available measurements and facilities and will be used as a basis for recommendations relative to the need for new measurements and/or facilities.

\subsection{Sensitivity Studies}

Quantification of the uncertainties in computed core physics parameters that result from propagation of uncertainties in the underlying nuclear data and other input parameters used in the various modeling codes is a key component of the quality assurance process for reactor physics modeling and simulation. It is also an important mechanism for quantifying the need for additional nuclear cross-section measurements and/or integral evaluations for VHTR and as a guide in the planning of future integral measurements and evaluations. Mathematically rigorous sensitivity and uncertainty analysis based on perturbation theory can, for example, be used to identify nuclides that contribute to calculational uncertainties and to quantify the propagated uncertainties in the context of the currently anticipated, likely VHTR core designs. Sensitivity coefficients are calculated by generalized perturbation theory codes and folded with multigroup covariance data (where available) to derive propagated uncertainties in computed integral reactor parameters arising from the nuclear data.

Integral parameters to evaluate include reactivity, peak power, reaction rate ratios, nuclide inventory, safety coefficients, etc. The impact of cross-section data uncertainty on the accuracy of each parameter is evaluated, along with the identification of nuclides, cross-section types, and energy ranges that have the greatest impacts on the accuracy of integral parameters. The process also can be used to rigorously quantify whether a given existing integral benchmark experiment is sufficiently similar to a contemplated VHTR system design to be of significant utility for validation of computations for the system being designed. 


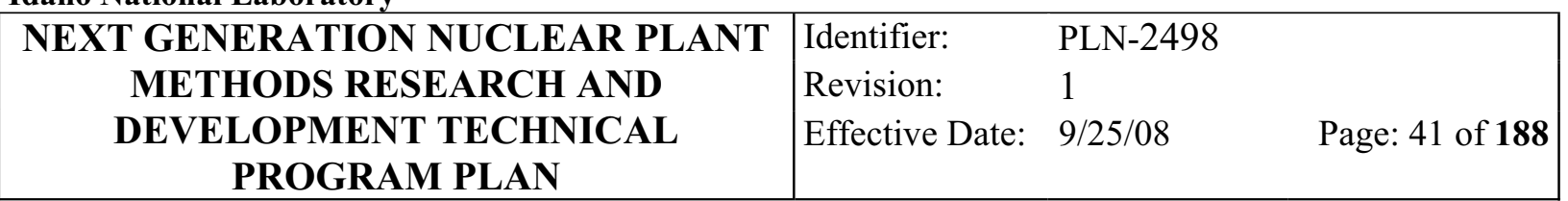

ANL has completed an initial sensitivity analysis for a prismatic concept for the VHTR, which, as is also the case for the pebble-bed concept, features a somewhat harder thermal neutron spectrum and a significantly higher fuel burnup target than is the case for standard LWRs. This study demonstrates a need for improved cross-sections for some isotopes, including ${ }^{238} \mathrm{U},{ }^{239} \mathrm{Pu},{ }^{240} \mathrm{Pu}$, and ${ }^{241} \mathrm{Pu}$, primarily in the resonance energy range, in order to satisfy the defined accuracy requirements on key computed integral parameters. Additional sensitivity studies conducted by ANL for other transuranic nuclides may be of similar importance for some of these concepts, although the primary needs appear to be similarly focused around a few plutonium and americium isotopes. The target accuracies identified in these studies are very stringent and, in many cases, will be a challenge to achieve.

As part of this program plan, the sensitivity studies conducted in FY 2007 and beyond for the prismatic VHTR concept will be extended to the pebble-bed concept. In the near-term, existing code suites will be used for this application. In the longer term, some neutronics code upgrades (for example, development of an adjoint capability for the PEBBED code for modeling of continuously-fueled (pebblebed reactors) will also be necessary as part of this task to allow the most realistic treatment of pebble-bed VHTR concepts. The capabilities to be developed in this program element will be needed both for studies of uncertainties driven by nuclear data and for various other types of analyses. Examples include analysis of multivariate uncertainty propagation in connection with integral data evaluations described in the previous section, studies of reactor kinetics parameters, and similarity studies useful for selection of pertinent integral experiments for $\mathrm{V} \& \mathrm{~V}$, etc.

\subsection{Differential Nuclear Data Measurements}

Essentially all integral nuclear parameters computed using modern reactor physics codes and data libraries are affected by propagation of uncertainty in the underlying differential nuclear data used in the computational models. These parameters include:

- Criticality (multiplication factor)

- Reactivity feedback coefficients (e.g., Doppler, Coolant Void)

- Kinetics parameters (e.g., Effective Delayed Neutron Fraction)

- Reactivity loss during irradiation (Burnup Swing)

- Peak power value

- Conversion ratio of sustainable cores

- Transmutation potential of burner cores

- Maximum displacement per atom (dpa), maximum helium and hydrogen-production, etc.

- Decay heat, radiotoxicity, and neutron and gamma radiation levels.

However, studies conducted to date under the Generation IV and Advanced Fuel Cycle programs have shown that the necessary cross-section information may not be available with the required accuracy from the current nuclear databases for some key nuclides of interest. The current VHTR design candidates have a somewhat harder thermal neutron spectrum than standard LWRs, a more complex fuel geometry, and a fuel cycle with two to three times the burnup. The sensitivity studies complete by ANL for a representative VHTR configuration indicate a need for improved cross-sections for some isotopes, including ${ }^{238} \mathrm{U},{ }^{239} \mathrm{Pu},{ }^{240} \mathrm{Pu},{ }^{241} \mathrm{Pu}$, primarily in the resonance energy range, in order to satisfy the defined accuracy requirements on key computed integral parameters. Integral reactor measurements have of 


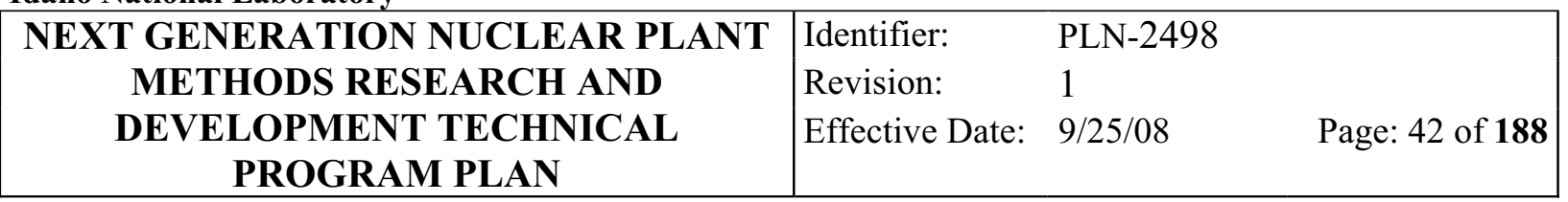

course played, and will continue to play, an important role in reducing the uncertainty of various actinide cross sections of interest for advanced reactor and fuel-cycle design. Key improvements in the relevant data can be achieved by various types of adjustments based on integral experiments, coupled with improved covariance information for the cross sections of interest. However, additional differential measurements are also likely to be important to the overall effort in some cases. Furthermore, there is a premium on application of innovative measurement approaches that offer improved accuracy in the context of very stringent requirements.

As a somewhat simplified but illustrative example of the current situation, Figure 12 shows a plot of the ENDF/B-VI data file values for the ${ }^{240} \mathrm{Pu}$ fission cross-section (the black solid line), along with available published direct measurements over broad energy ranges in the same experiment shown by the colored vertical lines, with the length of the line being an indicator of the reported uncertainty of the data. Experimental data below $\sim 10 \mathrm{eV}$ are limited to single-point experiments that may or may not have been performed under the same conditions. Thus, in several energy ranges of interest, the ENDF values are heavily based on theoretical models with limited experimental data input, and can be highly uncertain. It should also be noted that even where data are available the reported uncertainties are non-negligible as in the capture cross section for ${ }^{240} \mathrm{Pu}$ (Figure 12b). This capture cross section is of particular importance because neutron capture in ${ }^{240} \mathrm{Pu}$ leads to ${ }^{241} \mathrm{Pu}$, which has a large (but also uncertain) fission crosssection, as well as a large capture cross section. Such uncertainties in fundamental nuclear data can propagate in a manner that can have significant effects on the uncertainty of some computed safetyrelated and operational reactor parameters.
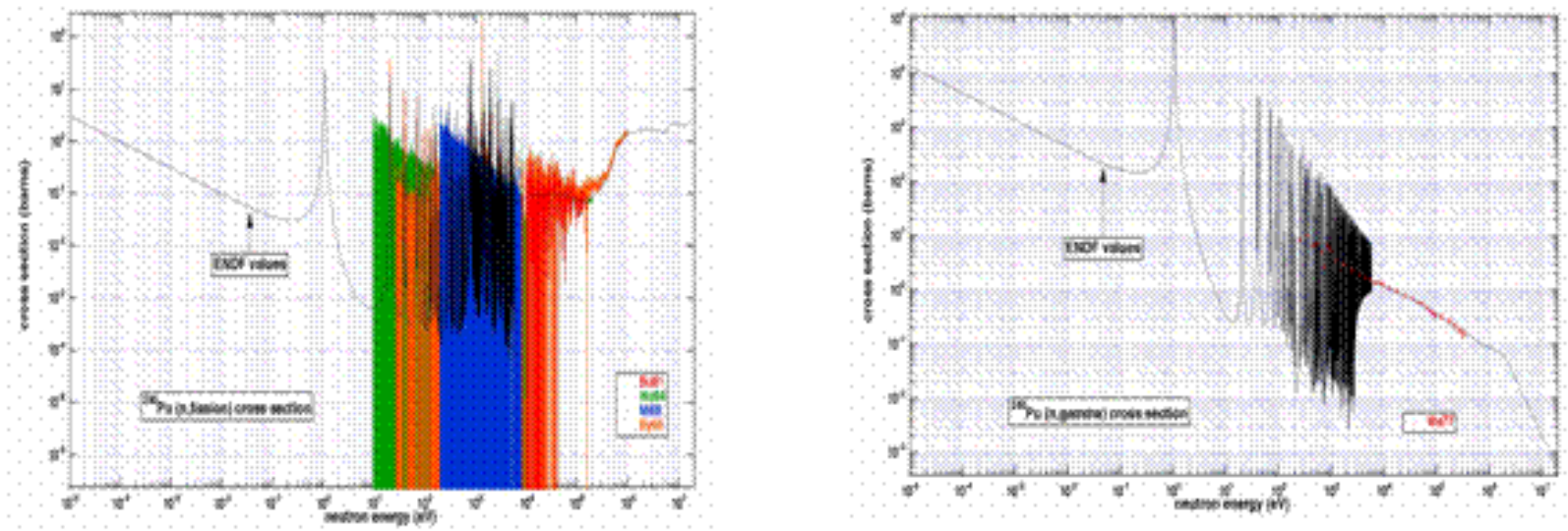

Figure 12. Fission (left) and capture (right) cross sections for ${ }^{240} \mathrm{Pu}$. ENDF/B. Version 6 evaluations are shown with key original data superimposed.

A comprehensive standard data base, CINDA (Computer Index of Neutron Data), maintained by the National Nuclear Data Program at Brookhaven, was used as the source for experimental data files and references for ${ }^{240} \mathrm{Pu}$ shown in Figure 12. In a search of CINDA, 1,450 references and data files were found, but not all of them are reporting experimental results, as these references include papers on evaluations, theoretical calculations and models, and papers without data values. Of these, only one direct measurement of the neutron capture cross section over an extended energy range under self-consistent conditions was found. All other capture cross-section information was extracted from ratio measurements relative to other nuclides, based on calculational extractions from total neutron induced reactions on a ${ }^{240} \mathrm{Pu}$ sample, or composed of single point measurements at one energy or averaged over an energy range 


\begin{tabular}{|c|lll|}
\hline NEXT GENERATION NUCLEAR PLANT & Identifier: & PLN-2498 \\
METHODS RESEARCH AND & Revision: & 1 & \\
DEVELOPMENT TECHNICAL & Effective Date: & $9 / 25 / 08$ & Page: 43 of 188 \\
PROGRAM PLAN & & & \\
\hline
\end{tabular}

to yield a single value. The vast majority of the single point values were at thermal energies or were integral values.

The summary point is that the roughly 50,000 points in the ENDF data file for the ${ }^{240} \mathrm{Pu}$ capture cross section are the result of one or more nuclear model calculations with rather limited experimental data as input. There are 18 experimental data files-18 experimental references in the 1,450 CINDA references - that represent any experimental measurement, with only one file containing a direct measurement with experimental results over an energy range. The 17 other experimental data files used in compiling the ENDF file are total cross-section measurements, ratio measurements, or single-point measurements. As another example, there are 810 references in the CINDA database for the ${ }^{240} \mathrm{Pu}$ fission cross section. Of these references, 40 have experimental data of some form that are used to construct the ENDF evaluated file containing 50,546 data points. The four experimental data files that are plotted represent the only direct, multipoint measurements of the cross-section out of the 40 references containing experimental cross-section values. The other 36 references of experimental data sets are either ratio measurements, single-point measurements, or average values over several broader energy ranges. The single-point values are Maxwellian distributions about some central energy values, generally $0.025 \mathrm{eV}$. The $810{ }^{240} \mathrm{Pu}$ fission cross-section references in CINDA also contain experimental data on other parameters (nu, yield, average kinetic energy) associated with fission as well as evaluations, theoretical papers, reports, and other works that do not contain direct data. Note that the situation for ${ }^{242} \mathrm{Pu}$ is similar to that described in greater detail above for ${ }^{240} \mathrm{Pu}$.

Because the minimum lead time for the type of measurements and subsequent evaluation required is on the order of 5 years, it is important that nuclear data improvement activities pertinent to VHTR applications be initiated in the near term so that the necessary information for the VHTR design will be available in a timely manner. Some new measurements for the key cross sections of interest for VHTR applications are in fact currently being conducted by Los Alamos National Laboratory under the Advanced Fuel Cycle Program. Additional measurements in the resonance energy range may be conducted at the Oak Ridge ORELLA Facility under the DOE-NNSA National Nuclear Criticality Safety Program some time in the future. The results of such measurements will ultimately be incorporated into the standard ENDF/B files via the Cross Section Evaluation Working Group, coordinated by the U.S. National Nuclear Data Center at Brookhaven National Laboratory. The new information may also be incorporated into other standard cross-section databases such as the European JEF file, or the Japanese JENDL files.

Under this portion of the planned VHTR Design and Safety Methods Validation program, INL, in partnership with ANL and various university and international collaborators, is in an excellent position to significantly contribute to addressing the need for new nuclear data via the performance of complementary measurements for the actinides of interest at the ANL Intense Pulsed Neutron Source (IPNS). Such measurements are important to confirm measurements performed elsewhere and to reduce the uncertainty in the final ENDF evaluated libraries by providing additional, independent, data sets.

Over the past several years, the INL Nuclear Physics Group has installed an array of detectors, the supporting electronics, and a data acquisition system based on techniques developed over the last two decades in nuclear physics at the IPNS, and has been using this array for the study of fundamental aspects of the nuclear fission process. This work and related previous work has produced over 100 refereed journal papers and established an international collaboration to support the experimental effort through data analysis. In the past 3 years, INL has also undertaken an effort to upgrade the system in a manner that allows measurement of absolute nuclear cross sections, specifically to support VHTR needs. 


\begin{tabular}{|c|lll|}
\hline NEXT GENERATION NUCLEAR PLANT & Identifier: & PLN-2498 \\
METHODS RESEARCH AND & Revision: & 1 & \\
DEVELOPMENT TECHNICAL & Effective Date: & $9 / 25 / 08$ & Page: 44 of 188 \\
PROGRAM PLAN & & & \\
\hline
\end{tabular}

The INL/ANL measurement program at IPNS would be coordinated with related efforts elsewhere via the Gen-IV/Advanced Fuel Cycle Physics Working Group, especially in the case of nuclear data measurements under way at the Los Alamos Neutron Science Center (LANSCE) under the Advanced Fuel Cycle program as previously noted. Because of the different and independent approach used for measurements at IPNS, the work can complement, not duplicate, those efforts, and it can result in substantial contributions to the national and international nuclear database required to support the VHTR design in the near term and the overall Generation IV advanced nuclear energy program in the longer term. Furthermore, the INL effort would take advantage of the potential for leveraging of funds in the case where VHTR data needs coincided with data needs that will emerge in the context of the new Global Nuclear Energy Partnership (GNEP). Further details of the specific INL/ANL measurement capabilities and planned work scope are provided in Appendix C.

\subsection{Summary: Focus and Direction}

Developing and reviewing VHTR-specific integral data evaluations and defining new experiment requirements, as prioritized by emerging programmatic needs, is high priority. As noted in Section 4.1, these activities will be conducted within the framework of the IRPhEP, which will expedite the overall $\mathrm{V} \& \mathrm{~V}$ process via standardization, international collaboration, and up-front facilitation of compliance with regulatory requirements.

Development of specifications for a sensitivity study appropriate to the pebble-bed design concept for the VHTR, as noted in Section 4.2, are high priority as is the need to determine the computational requirements instrumental in completing this study. Sensitivities to various isotope uncertainties will likely be somewhat different for the pebble-bed design compared to the prismatic design, primarily because of the different asymptotic fuel loading pattern that will be attained. In addition, sensitivity analysis capabilities that would be suitable for this application, implement this capability, and initiate the necessary computational studies that need to be assessed in the near term.

Beyond the R\&D identified in the above two paragraphs, a baseline pebble-bed modular reactor (PBMR) sensitivity study, in collaboration with ANL, needs to be performed as soon as possible (FY 2008). Concurrently, collaborative ANL/INL studies should be performed of uncertainties and sensitivities in VHTR system parameters for both concepts (prismatic and pebble-bed) as the system designs evolve, focusing on a single concept when the decision is made regarding whether the NGNP will be a prismatic or a pebble-bed type system.

In later years, collaborative ANL/INL studies of uncertainty propagation and sensitivities in VHTR system parameters for the selected system concept should be continued. A high-level time line of the nuclear data R\&D is shown in Figure 13. 


\begin{tabular}{|c|c|c|}
\hline $\begin{array}{l}\text { NEXT GENERATION NUCLEAR PLANT } \\
\text { METHODS RESEARCH AND }\end{array}$ & $\begin{array}{l}\text { Identifier: } \\
\text { Revision: }\end{array}$ & $\begin{array}{l}\text { PLN-2498 } \\
1\end{array}$ \\
\hline $\begin{array}{l}\text { DEVELOPMENT TECHNICAL } \\
\text { PROGRAM PLAN }\end{array}$ & Effective Date: & $9 / 25 / 08$ \\
\hline
\end{tabular}

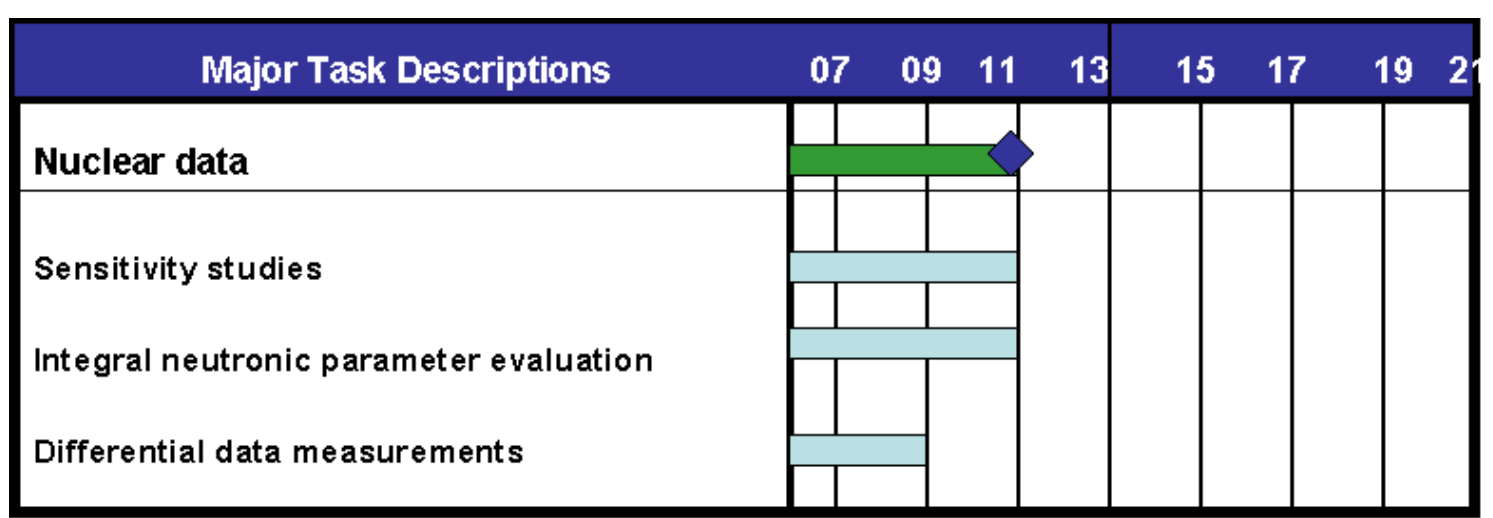

\section{Task completion}

Figure 13. Nuclear data schedule. ${ }^{j}$

j. Subsequent schedules do not include the proposed differential data measurement work described in this chapter and in Appendix C. 


\begin{tabular}{|c|lll|}
\hline NEXT GENERATION NUCLEAR PLANT & Identifier: & PLN-2498 \\
METHODS RESEARCH AND & Revision: & 1 & \\
DEVELOPMENT TECHNICAL & Effective Date: & $9 / 25 / 08$ & Page: 46 of 188 \\
PROGRAM PLAN & & & \\
\hline
\end{tabular}

\section{NEUTRONICS AND REACTOR KINETICS ANALYSIS DEVELOPMENT}

In this chapter the needs for new or enhanced neutronic methods are identified. This chapter evolved from the previous versions and many of the ideas previously presented are still present, and therefore are reiterated and confirmed as part of the current goals. Other ideas, present in previous versions of this document, are still mentioned, but only in order to preserve the historical train of thought and illustrate the many alternatives that had to be winnowed down. These latter ideas and approaches are no longer considered part of the plan to be implemented.

The neutronic design and the analysis of the operation of the NGNP requires the availability of core analysis tools (codes and data) for use in a number of necessary tasks. These tasks include (1) cross section preparation and fuel assembly lattice calculations to produce effective nuclear parameters for subsequent whole-core analysis, (2) static reactor analysis for core design and fuel management, and (3) reactor kinetics and safety analysis. In addition to these three traditional areas of neutronic analysis, supporting studies will have to be carried out on (4) core heating and shielding calculations and (5) decay heat calculations. Finally, the novel high temperature and high burnup aspects of the NGNP will require a sixth area to be considered: (6) evaluating the impacts of material-neutronics interactions on core performance and consequently on core design. The codes must also be qualified for use in safety evaluations. A recent review of existing state-of-the-art diffusion and transport whole-core analysis capabilities indicated that the codes containing R-Z (R- $\theta-Z)$ and Hexagonal-Z geometry options could be used for VHTR modeling. However, there are certain features of the NGNP design that require modifications to the available capabilities. Nearly all the advanced reactor physics tools in the U.S. nuclear industry were developed for the analysis and design of light-water reactors (LWRs) - the dominant reactor types in the U.S. There are, however, significant differences between the LWR and the NGNP designs that make the direct application of the LWR tools inadequate for the analysis of the NGNP. The physics characteristics of the NGNP are quite different from those of the commercial LWRs. The differences include (1) an annular core design, (2) solid graphite moderator, (3) higher enrichment of the uranium fuel, (4) TRISO particles-based fuel, (5) helium gas coolant, (6) large temperature rise/gradient across the core, (7) large holes for guiding control rods in fuel and radial reflector positions, and (8) planned operation to significantly higher burnup levels than in LWRs. A few modern codes were also developed for the analysis and design of liquid metal-cooled fast breeder rectors (FBR). The same limitations as for the LWR codes make these inadequate to the analysis of the NGNP.

The heterogeneity effect arising from the use of the TRISO fuel particles (termed double heterogeneity effect) must be adequately treated in the lattice physics code(s) utilized for the analysis of the NGNP in order to obtain accurate results for the core criticality state. This effect has been found to be about $2-4 \% \Delta \mathrm{k} / \mathrm{k}$ (reactivity) in NGNP assemblies/cores using enriched uranium fuels (Terry 2001), and about $10-15 \% \Delta \mathrm{k} / \mathrm{k}$ for those using transuranics fuels as in the deep-burn concepts (Plukiene 2003). Additionally, the NGNP core is neutronically small because of low neutron absorption in the graphite moderator (which leads to a long mean free path for neutrons) and the annular core design. This poses a challenge to the neutronic codes that are based on homogenized few group constants. Consequently, more energy groups have to be used than for conventional LWR analyses, and multi-assembly, partial-core calculations, or other re-homogenization and spectral iteration procedures might be needed for generating accurate homogenized cross sections and correction factors. The lattice physics code should therefore be able to provide adequate capabilities for these calculations. This includes the necessity for such codes to be extremely fast and compact while retaining high fidelity in order to allow embedding their functions within the full core global computations as well as within fuel management (depletion) and optimization 


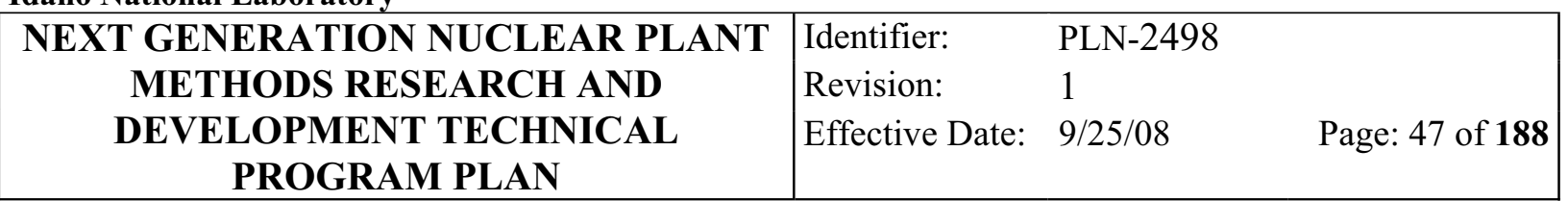

applications. The combined spatial homogenization - energy groups collapsing (or condensation) steps and whole core models - must be capable of correctly and consistently treating control rods and burnable poison in off-center and non-symmetric locations. The whole-core analysis tool should be able to very accurately model the core reactivity, flux, and power distributions, which accounts for the severe flux gradients and power peaking at the core and reflector interfaces of the annular core and for the very high burn-up in the fuel. Local neutron streaming effects in the axial direction arising from the control rod holes in prismatic VHTR designs and the wall effects in pebble-bed design should be accurately represented. The core depletion state (including the nuclide number densities and core burnup distribution) should also be accurately predicted. These core physics parameters have a direct impact on thermal-fluids/safety analysis, fuels and materials designs, and plant economics. Additionally, the code suite should be computationally efficient in order to perform the large number of calculations required to support core scoping analysis and detailed designs in a reasonable time.

A heterogeneous whole-core transport capability using a stochastic or deterministic transport theory solution method would be desirable for modeling VHTR cores accurately, eliminating the cumbersome and complex tasks of lattice cross-section generation, condensation, functionalization, local information recovery, etc. Currently, however, no such capability exists that has a detailed thermal feedback model, the depletion option, and can give whole-core solutions in reasonable time. Therefore, practical computational tools for VHTR design and analysis have, until now and out of necessity since a better scheme is unavailable, been based on the conventional two-step deterministic lattice and whole-core calculation approaches, which have been used successfully in the LWR industry. Such tools were also developed for and properly applied in the case of the earlier graphite-moderated, gas-cooled, reactors. However, their usefulness in the case of the NGNP-type design is at best limited. The reason is that the approach to passive safety goal for the NGNP imposes a very thin annular core design and hence a situation in which inter-zone spectral effects (i.e., spectral interpenetration between zones) are very significant and cannot be ignored during the diffusion theory data preparation, rendering the classical two-step approach inappropriate. Therefore, the prismatic NGNP will require an approach that incorporates the "in-context" derivation of diffusion theory data, as already done through spectral iterations in the PEBBED and V.S.O.P. codes now used for the pebble-bed design and analysis. Such an approach could be implemented into existing code suites or through the development of a new suite comprising a mix of existing codes and new portions of code. Examples of such a suite could be one based on DIF3D-VARIANT, PARCS, or HEXPEDITE. However, the amount of effort required for modification of existing codes and algorithms to achieve a level of fidelity comparable to the one achieved with the PEBBED suite for PBR modeling will be larger the more complex and comprehensive the starting set of codes is. It may prove to be more economical and faster to build most of the suite rather than modify existing codes, as was shown in the case of PEBBED. Ultimately, if time were not of the essence, the development of a transport theory-based modeling capability with detailed geometry and very fine energy-group-structure would be highly desirable. However, such a capability with the proper characteristics that would be responsive to the needs of the NGNP project is presently realistic. ${ }^{\mathrm{k}}$

The kinetics and dynamic modeling of the NGNP will also require codes that incorporate phenomena not usually expected for the LWR codes. Among the requirements that are to be imposed on kinetics and dynamic modeling codes for the NGNP is the incorporation of TRISO-level compatible temperature

k. Of course a transport theory capability with some the required features exists: the MCNP code is capable of very high fidelity both in geometric and energy detail. But the code is not practical for the frequent and repetitive scoping, optimization and the fuel cycle analyses required in the near phase of the NGNP project, and for the coupled neutronicsthermal-hydraulics applications that will be required for supporting the licensing activities anticipated in a later phase. Yet, the MCNP code is useful for benchmarking, although it also requires validation for the type of reactor under consideration, which is underway. 


\begin{tabular}{|c|lll|}
\hline NEXT GENERATION NUCLEAR PLANT & Identifier: & PLN-2498 \\
METHODS RESEARCH AND & Revision: & 1 & \\
DEVELOPMENT TECHNICAL & Effective Date: & $9 / 25 / 08$ & Page: 48 of 188 \\
PROGRAM PLAN & & & \\
\hline
\end{tabular}

feedback neutronic modeling, including Doppler feedback and Doppler-related material-neutronics interface effects, as well as material-neutronic interface and graphite moderator effects. Examples of such effects are those that can be induced by changes in physical thermal and lattice-nuclear properties during transients, which may arise because of temperature-induced annealing of radiation damage. Other examples are the Wigner energy, second Wigner energy, graphite scattering kernel effects, dislocation recovery, and the hysteresis phenomena that characterize these properties. Such effects are novel introductions to the physics of reactors and could be more easily implemented in new subroutines and new codes; however, at greater expense, they could also be implemented as modifications to legacy codes. The magnitude of these effects has not been fully ascertained. However, early indications point towards the necessity of their incorporation into reactor physics codes and models (Hawari 2007; Ortensi 2008).

Because of the large temperature rise across the core and the use of graphite moderator and helium coolant, the NGNP core reactivity responses to thermal upsets would be different from those of LWRs that use water as both the coolant and the moderator. These differences indicate that the verification and validation ( $\mathrm{V}$ and $\mathrm{V}$ ) bases that have been developed for LWRs would not be adequate for the NGNP and consequently effort must be devoted to the $\mathrm{V}$ and $\mathrm{V}$ of tools developed for the analysis of the NGNP. In addition, prior to adopting legacy codes for use in NGNP design and analysis and engaging in expensive modifications and $V \& V$ of said codes, an initial effort should be carried out to test their performance against realistic benchmarks and situations representative of the NGNP designs and to ascertain the feasibility and ease of the modifications they will require. The tests and benchmarks should particularly emphasize the features identified above, which require capabilities different from those used in LWR analyses. The assessment of the effort needed for the related code modifications should include an estimate of the complexity faced in implementing the changes and a realistic estimate of the financial cost and lead-time required in implementing the changes.

Physics tools were developed decades ago by the U.S. nuclear industry for the analysis of graphite moderated, gas-cooled reactors. However, they lack pedigree and sufficient modern V\&V for the NGNP annular core design within the operating ranges envisioned. These legacy tools could make many approximations because the earlier gas reactor designs contained Thorium-fertile and highly enriched Uranium fissile fuels resulting in different reactor physics characteristics and reactivity responses, at both low and high burnup states. These legacy tools cannot be easily adapted to the modeling of advanced NGNP cores. The plans for carrying out the needed development tasks are described in this section.

This section of the overall methods development plan focuses on the development of deterministic codes including spectrum codes, lattice physics codes, and nodal diffusion codes that can be used for efficient and accurate design of the NGNP or for verification of analyses performed using other methods and codes. In order to accomplish the project goal efficiently and in a timely manner, the most costeffective and time-responsive mix of existing and new codes will be used as the components of the new code suites. The legacy/existing codes will have to be augmented with the addition of required functionalities for VHTR applications. The two leading NGNP concepts under consideration at this time (prismatic and pebble-bed), are sufficiently different as to require separate but structurally similar development efforts. For applications to pebble-bed reactors with circulating fuel, INL is developing a complementary code suite around the PEBBED code. Although these codes provide the starting point of this project, a significant amount of development is required to enhance their capabilities and to equip them with group-constant generation modules that properly incorporate all of the relevant physics. The generation or modification of the relevant supporting codes was initiated in FY 2008. The principal of these supporting codes (COMBINE-7.1) is nearing completion as of this writing. The PEBBED-based suite has already been applied to pebble-bed annular cores and was proven effective. Current 


\begin{tabular}{|c|lll|}
\hline NEXT GENERATION NUCLEAR PLANT & Identifier: & PLN-2498 \\
METHODS RESEARCH AND & Revision: & 1 & \\
DEVELOPMENT TECHNICAL & Effective Date: & $9 / 25 / 08$ & Page: 49 of 188 \\
PROGRAM PLAN & & & \\
\hline
\end{tabular}

developments are aimed at enhancing the level of fidelity and flexibility of the suite. For the prismatic block reactors, INL is developing a suite that incorporates the features of the PEBBED suite by merely exchanging the neutronics solver for a hexagonal-z geometry module and making additional, related adaptations, to the cross-section generation and depletion modules. The motivation for choosing this path is that PEBBED has already been proven to work for NGNP-type HTRs. In parallel, ANL is building a suite centered on the DIF3D/REBUS-3 code, which has been successfully used for the physics analyses of fast reactor and light-water thermal reactor systems. Methods common to both reactor types will also be developed and applied accordingly.

Code suites, such as the ones prescribed and described in this section, are required for the range of reactor design and analysis applications necessary for the design optimization, verification, licensing, and eventual operation of the NGNP. For example, PEBBED was originally written for rapid scoping and conceptual design of PBRs in their long-term operating phase, with an asymptotic fuel-loading pattern. It is expected that a PBR will be at or near this asymptotic (equilibrium) burnup state for most of its operating life and PEBBED was written to converge directly upon, and optimize, this core state. As the NGNP program grew, however, the code was modified to capture more detailed and complex physics. It was coupled to the HTR-standard THERMIX heat transfer and gas dynamics code to provide more accurate temperatures for the cross-section feedback algorithm. The COMBINE spectrum code used to generate these cross sections was modified to better account for both the double heterogeneity of PBR fuel and the increased presence of low-energy resonances that can have a significant effect upon core reactivity and nuclide inventory at high burnup. An effort was also undertaken to develop a computational pebble flow model that simulates the pebbles' spatial distributions and local patterns, which can affect the coupling of the burnup and power profiles. The pebble flow model work resulted in the PEBBLES code that is being used for the analysis of the pebble-bed reactor response to earthquakes and in predicting the production of dust in operating reactors and the estimation of stresses imposed on individual pebbles and reactor reflectors and other structures. These efforts and others will enable PEBBED to model the PBR fuel cycle with a very high degree of fidelity.

In the area of reactor kinetics and dynamics for the NGNP, INL will adapt the RELAP5-3D code. However, since this code did not originally possess a cylindrical geometry neutronics capability, an interim solution had to be adopted for that version of the reactor. Therefore, in a first stage the Penn State transient code NEM (Nodal Expansion Method) was acquired, modified, and tested. NEM contains a cylindrical solver and an effort to couple it to the THERMIX code was also completed. The resulting product met with limited success as it inherited the limitations of its constituent codes.

Concurrently, with the NEM-related developments, an effort began at INL to create a kinetic (i.e., time-dependent) version of the static nodal analytic cylindrical geometry code being implemented into the PEBBED code (i.e., a new code based on the analytical nodal method contained within the PEBBED code was developed). The new code, CYNOD, has been completed and has been coupled to the THERMIX code and tested against some of the OECD PBMR400 benchmarks. The CYNOD code has also been recently coupled to the RELAP5-3D code, thus providing a state-of-the-art kinetics and dynamic capability for the analysis of the pebble-bed reactor, which will provide INL and the NGNP Project with a robust and quality-assured traceable safety/systems analysis capability suitable for licensing purposes. The RELAP5-3D-CYNOD code system has also been applied to one of the OECD PBMR400 benchmarks (rod ejection). The coupled code now requires further benchmarking and V\&V.

The combination of these tools (PEBBED-THERMIX-COMBINE-NEM and RELAP-CYNODCOMBINE) will provide the DOE, NRC, reactor vendors, and others with a comprehensive and modern suite of codes for both PBR conceptual design and licensing needs. For a prismatic version of the same 


\begin{tabular}{|c|lll|}
\hline NEXT GENERATION NUCLEAR PLANT & Identifier: & PLN-2498 \\
METHODS RESEARCH AND & Revision: & 1 & \\
DEVELOPMENT TECHNICAL & Effective Date: & $9 / 25 / 08$ & Page: 50 of 188 \\
PROGRAM PLAN & & & \\
\hline
\end{tabular}

capability INL will couple RELAP5-3D with a hexagonal-z kinetic solver that satisfies all the requirements for VHTR physics. For example, such a code could be PARCS (Downar et al. 2004), which has already been coupled to RELAP5 in some fashion. However, the modifications that would be required to adapt them to the specific physics characteristics of the NGNP may prove too technically complex to be practical. Nonetheless, an INL LDRD project is currently ongoing to explore the continued adaptation of the PARCS code to the NGNP physics. If that effort bears fruit, in all likelihood the resulting code will be adopted as the INL capability. In the meantime, two other efforts are envisioned that rely on the kinetic versions of the static and fuel management packages that are also being pursued. At INL, an option is the use of the existing kinetic version of the HEXPEDITE code (Fitzpatrick 1994). The advantage of this code with regard to its adaptability to the specific neutron physics characteristics of the NGNP has already been explained above.

All of the kinetic and dynamic codes discussed above will have to be subjected to extensive benchmarking and V and V. This is planned for the coming fiscal year (FY 2009) in the case of pebblebed codes (CYNOD and coupled RELAP5-3D-CYNOD). For the prismatic block design at ANL, efforts at benchmarking and deploying a fully compatible kinetics/dynamics capability will be delayed, pending the determination and resolution of the source of difficulty (Taiwo 2008) in attaining high fidelity in the underlying hexagonal-z neutronics solvers. It is expected that this effort should start in FY 2010. However it would start in FY 2009 if the ANL technical leaders determine that it is possible for ANL to perform this task in FY 2009 or if DOE mandates the earlier start. At INL the corresponding effort is ongoing, based on the HEXPEDITE code, which does not suffer the same problems in dealing with double heterogeneity and the singular control and reactivity hold-down features. The corresponding benchmarking is expected to start in FY 2009.

In summary, the demands of the PIRT and documents such as the Powers Report, NRC Draft Gap Analysis report (Ball et al. 2008), Moorman report (Moorman 2008), and above all the actual physical characteristics of the reactor design impose a set of requirements on the abilities of the codes to model certain phenomena and features. A comprehensive review of the identification of these requirements and the physics considerations that led to them are the subject of an INL report currently in preparation. A companion report will identify the actual modeling steps and methodologies envisioned at INL and determined necessary to meet all the requirements. The report will clarify the various trade-offs between using legacy codes versus developing new ones. The principal physics-based needs for the code suite were discussed above and are listed again here for convenience:

- Double heterogeneity

- Annular core design (spectral effects, long diffusion length, "neutronically thin" core)

- Burnable poisons (Complex geometry effects; non-traditionally homogenized)

- Off-center control rods and channels (if current GT MHR design is retained)

- TRISO-particle-level temperature feedback

- Material-neutronic material damage feedback mechanism for graphite (and to a lesser extent silicone carbide $[\mathrm{SiC}]$ )

- Microscopic depletion requirement with burnup and spectral context corrections

- Very high burnup levels.

Major development areas for NGNP physics methods have been identified. The following is a brief summary of the areas. 


\begin{tabular}{|c|lll|}
\hline NEXT GENERATION NUCLEAR PLANT & Identifier: & PLN-2498 \\
METHODS RESEARCH AND & Revision: & 1 & \\
DEVELOPMENT TECHNICAL & Effective Date: & $9 / 25 / 08$ & Page: 51 of 188 \\
PROGRAM PLAN & & & \\
\hline
\end{tabular}

Assembly Lattice Calculations. Existing modern codes were developed for reactor types with different physics (LWR or fast reactors). Codes developed specifically for graphite-moderated thermal reactors rely on techniques and assumptions that have not been updated in decades. Enhancements and improvements in modeling capability are needed to address unresolved issues in pebble-bed and prismatic high-temperature graphite-moderated reactor physics. One of the most important of these issues is the proper preparation of multi-group nuclear cross-sections suitable for subsequent use in the simulation of graphite-moderated, helium-cooled reactor core behavior. As explained in the specific task descriptions below, existing cross-section preparation methods for graphite-moderated reactors may yield poor agreement with continuous-energy Monte Carlo calculations in the thermal and epithermal energy ranges if the double-heterogeneity of the fuel form and assembly leakage are not carefully addressed. Improved treatments are needed since the basic reactor operating parameters are highly sensitive to any inaccuracies in the effective cross sections in this energy range. An imperative requirement is the proper treatment of configurations in which the reactor core is neutronically optically thin. The technical solution that was adopted for solving this problem in the PEBBED and V.S.O.P. codes and proved to be successful is that of spectral iterations between the lattice physics and the whole core computations. For the pebble-bed concept the lattice code is COMBINE (Grimesey et al. 1991). For the prismatic-block concept, specific, geometrically-exact codes are needed and current codes may be further developed. For the prismatic block concept geometrically correct lattice code is needed and current codes may be extended (DRAGON, HELIOS, explicit-transport-extended COMBINE etc.). Dancoff factors must be used to explicitly treat the particle-level heterogeneity; the INL/TU-Delft prisDAN code is being developed and tested and will be incorporated into COMBINE and the prismatic lattice method chosen.

Static Core Simulation, Core Design, and Fuel Management: Core simulators provide estimates of the core reactivity, flux and power distributions, and isotopic distribution data under steady-state conditions at specified points during the fuel cycle and also under transient conditions. Such data are required for safe and economic design and operation of reactors. Specialized core simulation techniques and codes are also required for the modeling of the power deposition. The proper accounting for production and transport of gamma photons is usually beyond the capabilities of a fuel management code, but it should be included as part of the analysis code suites. The power deposition distribution can differ significantly from the fission rate distribution due to the transport of gammas away from their creation sites.

For the pebble-bed reactor, the continuous movement and recirculation of fuel require solving simultaneously the diffusion and depletion equations in a way that most codes cannot model since they have stationary fuel as their assumed configuration. The PEBBED code is equipped to handle fuel movement and possesses an advanced genetic algorithms optimization feature. Additional development of PEBBED could involve modeling pebble discharge conus and approach-to-asymptotic-equilibrium core.

For the prismatic designs, INL is developing an alternate version of the PEBBED methodology that uses a hexagonal-z solver. The ANL codes DIF3D/REBUS-3 provide a core simulator capability; however, additional development would be needed, such as:

- Data preparation for annular cores

- Data preparation for strong local heterogeneities

- Thermal feedback

- De-homogenization models appropriate for high-temperature thermal reactors

- Fuel management and optimisation techniques. 


\begin{tabular}{|c|lll|}
\hline NEXT GENERATION NUCLEAR PLANT & Identifier: & PLN-2498 \\
METHODS RESEARCH AND & Revision: & 1 & \\
DEVELOPMENT TECHNICAL & Effective Date: & $9 / 25 / 08$ & Page: 52 of 188 \\
PROGRAM PLAN & & & \\
\hline
\end{tabular}

Reactor Dynamics: Safe operation of a power plant is intrinsically linked to time-dependent behavior of the coupled power plant system (core plus balance of plant). Because of the strong temperaturedependence of the core materials and the large temperature gradients that exist in these cores, the dynamics of the VHTR core can only be analyzed properly with coupled neutronic and thermal-hydraulic computations. Although transient neutronics and thermal-hydraulics codes have been available for decades, only in the past decade have the computers become capable of high-resolution coupled solutions of neutron transport and fluid dynamics. So-called multi-physics codes bear the promise of allowing analysts to probe complex reactor dynamics with some high degree of fidelity. Until such codes are finally operational for the analysis of full reactors, evolutionary codes from the current state of the art will have to be used. Current generation codes are under development at INL in the context of the NGNP project. The developments are discussed in this section.

For the analysis of pebble-bed reactor kinetics the CYNOD code is now available. CYNOD, the kinetic version of PEBBED, is coupled to THERMIX and to RELAP5-3D, both fully capable of NGNP analysis to the extent that ordinary analysis does not require a full CFD treatment. However, the coupled RELAP5-3D-CYNOD code still requires further benchmarking and V\&V. Another code worth considering for coupling to the neutron kinetic is the GRSAC code, although at this point no plans for carrying out the necessary coupling are being drawn.

For the kinetic analysis of prismatic block reactors, the DIF3D-K code, a kinetic version of DIF3D could be used, but it is coupled to the SAS code for thermal-hydraulic feedback, which may not be fully adequate for the modeling of the prismatic NGNP without further modifications (Taiwo 2008). A viable alternative is the use of RELAP5-3D with either the existing kinetic version of the HEXPEDITE code or the PARCS code. The latter two alternatives will be explored within the NGNP project.

For detailed kinetic analyses in situations with detailed geometric features, coupling to CFD codes may be necessary in order to provide correct feedback data to the neutron kinetic codes. New multiphysics tools are being developed for ultra-high fidelity simulation. An example of such a development is the effort to couple the 3D transport code EVENT with a new and efficient CFD code (PCICE-FEM) presently underway at INL. In the interim, a CFD module incorporated within the RELAP5-3D code should be used.

Material-Neutronic Interface: The effect of the radiation damage on core structures is of particular concern for both prismatic and pebble-bed designs. Graphite exhibits complex neutron scattering behavior under irradiation that includes changes to its thermal and neutronic properties over time. Current graphite and silicon carbide damage models are based upon empirical studies or very limited theoretical assumptions that may be unsuitable for VHTR operating conditions. Furthermore, high-temperature annealing (during either normal operation or accident conditions) may have rapid feedback effects and thus should be characterized as part of a comprehensive safety analysis. The U.S. DOE is separately supporting (NERI project) an effort to develop a model of radiation damage based upon first principles and to quantify the effects of damage under expected conditions. The NGNP project R\&D plan should include provisions for incorporating the outcome of that study into the NGNP reactor dynamics codes

TRISO-Level Feedback Mechanisms: The structure of the TRISO particle confers upon its physical, thermal and neutronic properties that are interrelated and significantly different from those of fuel pins in LWRs. The characteristics of the TRISO particle must form the basis of the methods that are used for evaluating the temperature and the materials effect feedback mechanisms. Preliminary results at INL have shown that such effects cannot be treated with legacy codes and that their modeling must be incorporated explicitly in both the neutronic and the thermal-hydraulic components of the suite of codes. 


\begin{tabular}{|c|lll|}
\hline NEXT GENERATION NUCLEAR PLANT & Identifier: & PLN-2498 \\
METHODS RESEARCH AND & Revision: & 1 & \\
DEVELOPMENT TECHNICAL & Effective Date: & $9 / 25 / 08$ & Page: 53 of 188 \\
PROGRAM PLAN & & & \\
\hline
\end{tabular}

Benchmark Evaluation, Uncertainty and Sensitivity Analysis, Validation and Verification: Ultimately all the codes and methods described above must be subjected to a rigorous testing and formal evaluation process involving: experimental validation, theoretical verification, code-to-code comparisons, and formal documentation that can meet licensing requirements. Such analyses must be performed to ascertain the accuracy of the results produced and to quantify the uncertainty therein-necessary steps in the licensing of a new reactor.

The work described herein begins the process of completing the suite of analysis methods to permit the full scope of NGNP design analysis calculations to be performed with state-of-the-art tools. An integral part of the development and testing of the new capabilities will be the assessment of their implications for NGNP design limits. INL, supported by ANL, will be leading the research efforts for these tasks. The two laboratories are cooperating on the identification and development of the code suites that incorporate the techniques required for accurate analyses of all current candidate NGNP concepts. These labs have already established working relationships with a number of universities and international organizations that have an interest in gas reactors. A number of workshops and electronic information exchanges have taken place and more are planned as the overall NGNP project proceeds. It is anticipated that the bulk of the code development effort will be completed in the first 5 to 6 years of the overall effort. After that, code maintenance, validation, and application to the ongoing NGNP design effort will continue for the duration of the project.

The following sections describe in detail the five major R\&D areas described above; specifically:

- Assembly Lattice Cross-section Generation

- Core Simulation and Design

- Reactor Kinetics and Power Plant Dynamics

- Neutronic-Material Interface

- TRISO-Level Feedback Mechanisms

- Validation and Verification.

\subsection{Unit Cell and Assembly Analysis for Cross Section Generation}

For NGNP applications, both the prismatic (DIF3D/REBUS system, HEXPEDITE, PARCS, etc.) and pebble-bed (PEBBED) codes require cross-section data preparation using specialized techniques that are not implemented in current software in the form that is needed. Cross sections used by PEBBED and DIF3D are calculated externally and passed to these global reactor analysis codes as input. For PEBBED simulations (and DIF3D analyses of the New Production Reactor), these cross sections were calculated by the INL's COMBINE code or by MICROX-2. COMBINE has been substantially modified and support codes have been created for it. The augmented COMBINE and the Dancoff factor codes developed for it have displayed good performance against critical benchmarks.

As illustrated in Figure 14, thermal neutron spectra in graphite moderated reactors have higher characteristic average energies than is the case for water moderated reactors, especially at the high (900$1000^{\circ} \mathrm{C}$ ) operating temperatures that are anticipated for the NGNP. As a result, special methods are required to account properly for self-shielding of resonances in the thermal energy range. This becomes particularly important in situations with high burnup because of neutron upscattering into the prominent low-lying resonances in plutonium. Core reactivity, temperature coefficients and other related phenomena are all highly dependent on proper modeling of resonance effects. The required computational 


\begin{tabular}{|c|lll|}
\hline NEXT GENERATION NUCLEAR PLANT & Identifier: & PLN-2498 & \\
METHODS RESEARCH AND & Revision: & 1 & \\
DEVELOPMENT TECHNICAL & Effective Date: & $9 / 25 / 08$ & Page: 54 of 188 \\
PROGRAM PLAN & & & \\
\hline
\end{tabular}

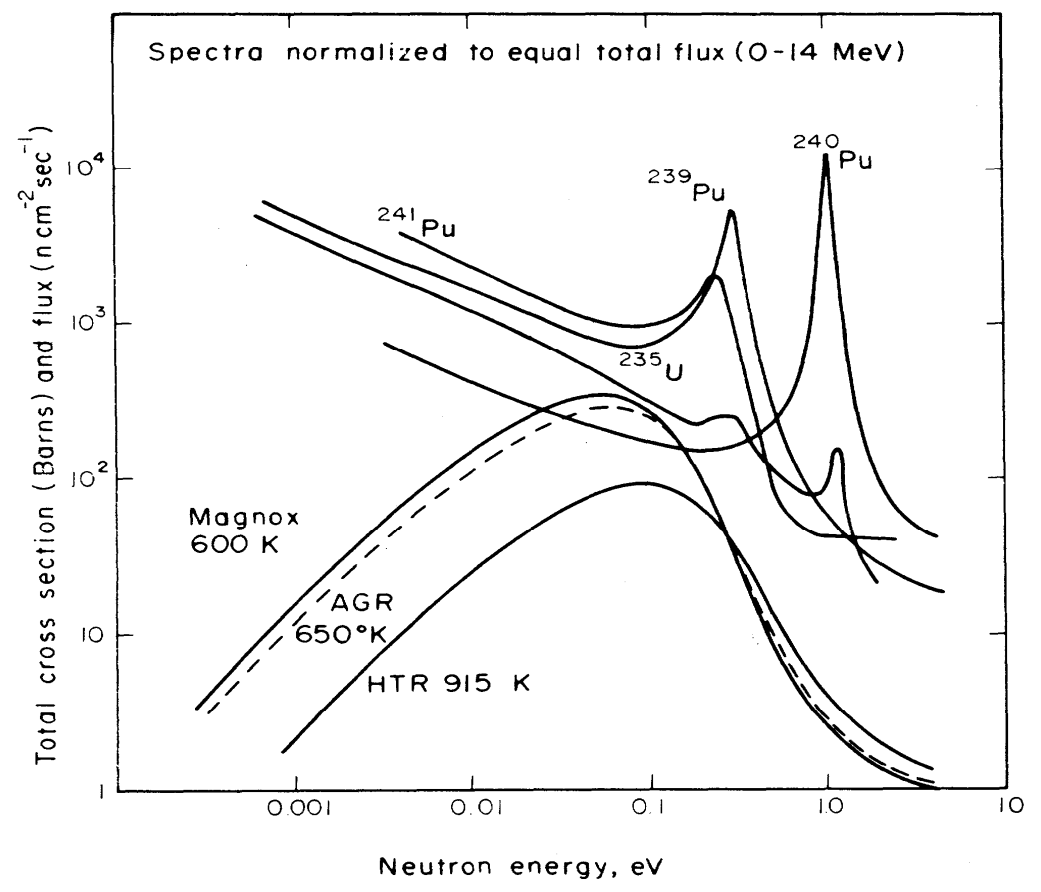

Figure 14. Typical HTGR spectrum and some low-lying resonances.

improvements will thus be developed under this plan and implemented into the spectrum codes as appropriate. Furthermore, the random distribution of fuel kernels within a compact or pebble is not accurately treated in any of the available codes with the exception of COMBINE with its pebDAN and nearly completed prisDAN support codes, which generate Dancoff factors that take random distribution of TRISO particles into account. Ignoring the randomness adversely affects the accuracy of the allimportant resonance shielding calculations that are required to produce accurate cross sections.

Finally, note that preparation of required cross-section libraries from the basic nuclear data files also involves very sophisticated data processing prior to application of the spectrum and assembly crosssection generation calculations described above. Under this R\&D Plan, INL will also maintain the necessary expertise and software tools required for this step in the overall reactor physics analysis sequence. This will include active participation in the National Nuclear Data Center's Cross Section Evaluation Working Group (CSEWG), as well as in corresponding international organizations whose focus is on the key interfaces between basic nuclear data measurements and the final ENDF files that have been evaluated and released for use in subsequent reactor physics applications.

\subsubsection{Method for Improved Treatment of Double Heterogeneity Using Improved Dancoff Factors}

An important aspect of improving cross sections is to account better for the heterogeneity on two scales in the NGNP: on a fine scale associated with the fuel particles, and on a more coarse scale associated with the pebbles or fuel compacts. Self-shielding and shadowing effects are important and must be accounted for on both scales. In the continuous-energy Monte Carlo code MCNP, it is possible to model every single fuel grain in the reactor, using the repeated structures feature. Spectral indices, as computed using MCNP, are listed in Table 5. 


\begin{tabular}{|c|c|c|c|}
\hline NEXT GENERATION NUCLEAR PLANT & Identifier: & PLN-2498 & \\
\hline METHODS RESEARCH AND & Revision: & 1 & \\
\hline $\begin{array}{l}\text { DEVELOPMENT TECHNICAL } \\
\text { PROGRAM PLAN }\end{array}$ & Effective Date: & $9 / 25 / 08$ & Page: 55 of 188 \\
\hline
\end{tabular}

Table 5. Spectral indices as computed by MCNP and MICROX-2.

\begin{tabular}{|c|c|c|c|c|}
\hline & & & \multicolumn{2}{|c|}{ BCC $61 \%$} \\
\hline & & & cold & hot \\
\hline \multirow{3}{*}{$\rho^{28}$} & \multirow{3}{*}{$\begin{array}{l}\text { Epithermal-to-thermal } \\
{ }^{238} \mathrm{U} \text { captures }\end{array}$} & MCNP & 5.91 & 7.50 \\
\hline & & MICROX-2 & 6.77 & 9.23 \\
\hline & & $\operatorname{diff}(\%)$ & 15 & 23 \\
\hline \multirow{3}{*}{$\delta^{25} \times 10^{2}$} & \multirow{3}{*}{$\begin{array}{l}\text { Epithermal-to-thermal } \\
{ }^{235} \mathrm{U} \text { fissions }\end{array}$} & MCNP & 9.63 & 10.99 \\
\hline & & MICROX-2 & 9.99 & 11.34 \\
\hline & & $\operatorname{diff}(\%)$ & 4 & 3 \\
\hline \multirow{3}{*}{$\delta^{28} \times 10^{4}$} & \multirow{3}{*}{$\begin{array}{l}{ }^{238} \mathrm{U} \text { fissions to } \\
{ }^{235} \mathrm{U} \text { fissions }\end{array}$} & MCNP & 28.08 & 30.14 \\
\hline & & MICROX-2 & 27.19 & 30.44 \\
\hline & & $\operatorname{diff}(\%)$ & -3 & 1 \\
\hline \multirow{3}{*}{$\mathrm{C}^{*}$} & \multirow{3}{*}{$\begin{array}{l}{ }^{238} \mathrm{U} \text { captures to } \\
{ }^{235} \mathrm{U} \text { fissions }\end{array}$} & MCNP & 0.360 & 0.463 \\
\hline & & MICROX-2 & 0.404 & 0.551 \\
\hline & & $\operatorname{diff}(\%)$ & 12 & 19 \\
\hline
\end{tabular}

However, in deterministic codes like PEBBED, HEXPEDITE or DIF3D, these features are accounted for in the cross sections they receive as input. Such cross sections must be generated using explicit modeling of the heterogeneity or using correcting factors that account for it (i.e., Dancoff factors). Therefore, the new method must also include the generation and incorporation of accurate Dancoff corrections for the effects of double heterogeneity.

Existing codes used for gas reactor analysis were developed without a full appreciation of the importance of randomness in particle distribution. Recent studies indicate the error introduced by assuming a regular array of fuel lumps is significant $(200-300 \mathrm{pcm})$. MICROX has been shown to differ significantly with continuous-energy MCNP calculations (Table 6). The treatment of randomness in DRAGON may also be inadequate. A rigorous treatment of randomness in the distribution of fuel lumps has been developed at INL and incorporated into the construction of Dancoff factors (completed pebDAN code for pebbles and evolving prisDAN code for fuel compacts). A similar treatment for explicit geometry lattice codes like DRAGON and HELIOS will also be explored.

\subsubsection{COMBINE Spectrum Code for Few-Group Cross Section Generation}

In the pebble-bed reactor, fuel elements (pebbles) move in a semi-continuous fluid-like manner through the core during operation. Recirculation of partially burned pebbles means that any pebble in the core is surrounded by pebbles with a wide range of burnup. Furthermore, the spectral history of each pebble is unique and can only be approximated (using a code such as PEBBED). Batch-loaded cores (LWR or prismatic gas-cooled reactor) generate cross sections from unit cell burnup calculations assuming fixed boundary and surrounding spectral conditions. This approach is not valid for the pebblebed reactor. Instead, cross section and core simulation calculations must be executed simultaneously and iteratively to obtain the proper burnup conditions in each spectral zone (the pebble-bed reactor analog to an assembly or block). Such coupling of the spectrum and core simulator codes has been performed between PEBBED and both MICROX and COMBINE. Zone leakage and temperatures from PEBBED are fed to the spectrum code to obtain cross-sections by interpolation among pre-computed data sets or 


\begin{tabular}{|c|lll|}
\hline NEXT GENERATION NUCLEAR PLANT & Identifier: & PLN-2498 \\
METHODS RESEARCH AND & Revision: & 1 & \\
DEVELOPMENT TECHNICAL & Effective Date: & $9 / 25 / 08$ & Page: 56 of 188 \\
PROGRAM PLAN & & & \\
\hline
\end{tabular}

online through direct calls to the code. Because the spectral zone itself is not clearly defined and contains a randomly packed assortment of pebbles, a geometrically rigorous spatial transport calculation (2 or 3dimensional) is neither wholly effective nor computationally efficient for the algorithm described above. A 1-dimensional (spherical) calculation with appropriate Dancoff factors and isotopics provided from PEBBED can yield cross-sections with the required accuracy, provided the spectral influence from the surrounding environment is properly taken into account. Although assemblies are not defined, spectral zones are, and the computations are performed on a spectral zone basis. Specifically the data, including TRISO-level and pebble-to-pebble Dancoff factors, are prepared for each spectral zone, taking into account the proximity of reflectors when warranted. In order to fully account for the spectral influence of neighboring spectral zone, it may be necessary to carry out spectral iterations between the spectrum code and the whole core model, as explained above. An alternative is the carry out whole core spectral correction steps, as discussed below.

In ongoing work, the INL spectrum code COMBINE is undergoing modifications to exploit the new Dancoff treatment and to incorporate a 1-D transport solution in order to account for mutual spectral influences of neighboring spectral zones. The incorporation of the 1-D transport module within COMBINE will near completion by the end of FY 2008 and will be finalized, tested, and benchmarked in FY 2009. When fully completed and tested, the new COMBINE code will replace the one already coupled within PEBBED. COMBINE solves the one-dimensional (spherical) B-3 approximation to the transport equation with Bondarenko treatment of unresolved resonances and Nordheim treatment of resolved resonances. It uses Dancoff input factors to correct the resonance calculation in the presence of distributions of fuel lumps and has options for self-shielding of cross sections in the thermal range. No geometry specification of an assembly or lattice is required and thus it is suitably fast and accurate for the online data generation for the PBR problem described above. Local isotopic content and temperatures of the local pebble distribution will be fed to COMBINE to generate self-shielded infinite-domain cell cross sections. The local spectrum strongly depends upon the inter-cell or inter-spectral zone current that is captured in COMBINE using buckling terms or with an embedded coupled spatial transport calculation. The finite-cell few group diffusion coefficients (including discontinuity factors to be coded in FY 2009) will feed the nodal diffusion calculation and burnup-dependent cross sections for depletion in individual pebble flow streams.

COMBINE has recently been modified by extending the Nordheim and Bondarenko resonance treatments fully into the thermal region while retaining the thermal self-shielding feature of the original thermal spectrum module. Results have compared favorably to analytic benchmarks and other calculations using MCNP (Figure 15), ANISN, and TWODANT. Further validation will be required for NGNP-specific configurations. A TRISO fuel benchmark for cross-section generation is being developed and will provide a basis for evaluating the accuracy of any codes identified for PBR analysis. Whenever possible, results will also be compared to corresponding MCNP models and experimental benchmarks. The newest version of COMBINE, to be finalized in FY 2009, goes beyond these changes and incorporates a 1-D transport solver for the treatment of spectral effects at interfaces between spectral zones, especially fuel-reflector interfaces. 


\begin{tabular}{|c|lll|}
\hline NEXT GENERATION NUCLEAR PLANT & Identifier: & PLN-2498 \\
METHODS RESEARCH AND & Revision: & 1 & \\
DEVELOPMENT TECHNICAL & Effective Date: & $9 / 25 / 08$ & Page: 57 of 188 \\
PROGRAM PLAN & & & \\
\hline
\end{tabular}

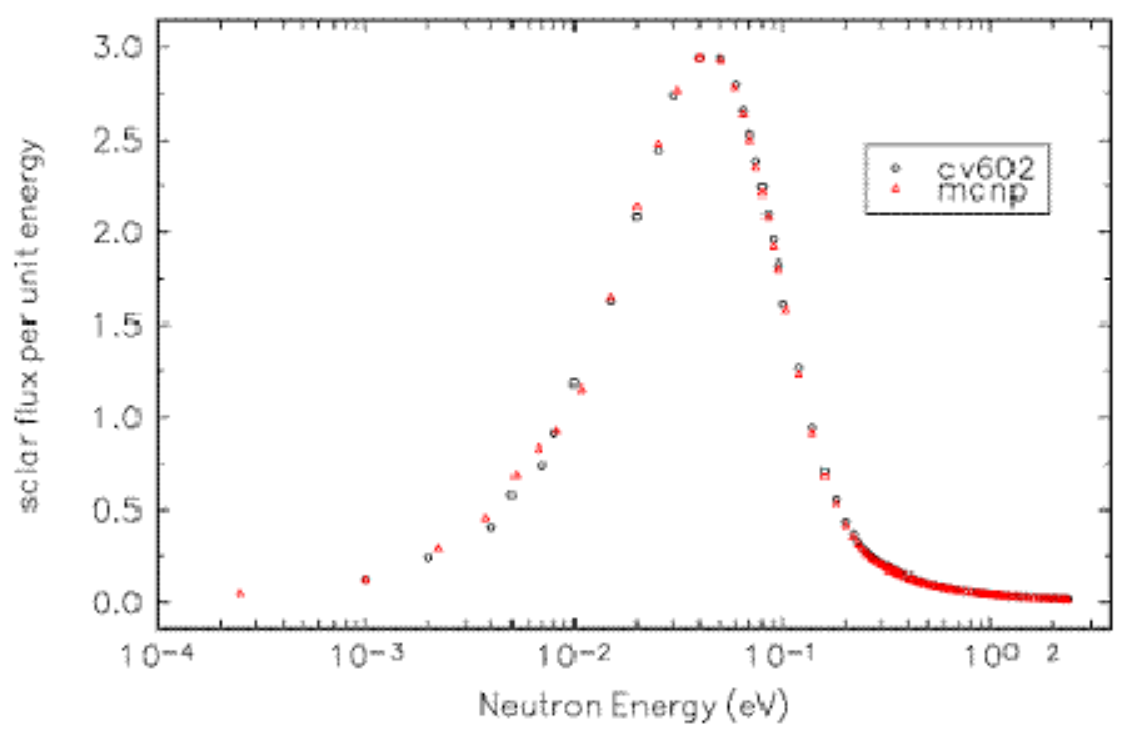

Figure 15. Thermal neutron spectrum computed by COMBINE (version 6.02) and MCNP for a PBMR268 unit fuel cell.

\subsubsection{Argonne National Laboratory Assembly Code for Prismatic Block Cross-section Generation}

In contrast to the random packing of the pebble-bed fuel, the prismatic reactor core is composed of hexagonal graphite blocks containing coolant channels and fuel compacts in ordered arrays. The compacts contain distributed randomly TRISO particles. A core simulator code, such as ANL's DIF3D, has as its basic computational element a hexagonal cell for which few-group diffusion coefficients must be computed by a lattice or assembly code once the basic unit cell cross sections have been determined. Previous analyses indicate that an under-prediction of about 3\% in k-infinity for a fuel element can occur if the fuel-graphite composite is treated as a homogenized mixture. Therefore, the lattice transport code to be used for group constant generation must be able to treat the double heterogeneity properly, and in addition must account for spectral variations across the basic lattice unit via appropriate neutron transport computations. This capability is available in a few lattice physics codes such as WIMS8, APOLLO2, DELIGHT, and DRAGON and will become available very soon within HELIOS. Where such capabilities exist, the codes (e.g., WIMS8 and APOLLO) are typically proprietary and are only available at great cost. In some cases, the source code is not available for release. The HELIOS code, in contrast, will be available to INL for the next 6 to 7 years without further licensing fees, and also available beyond that at a preferential fee if this option is exercised within the 6 to 7 years window.

The above considerations make the open-source DRAGON code an attractive option along with HELIOS, and for this reason further assessment and development of DRAGON will be a major ANL task in this project while INL will expend similar efforts on HELIOS. The DRAGON work will, as necessary and as possible, be done in collaboration with the researchers at the Ecole Polytechnique de Montreal who originally developed the code. ANL organized a first information exchange with the DRAGON code developers in February 2005. Preliminary investigation of the code performance in modeling the prismatic-type VHTR was also done. The study indicated that the code gives accurate values for the lattice eigenvalue, spectrum (neutron flux distribution in energy), fission rate distribution, and the double 


\begin{tabular}{|c|lll|}
\hline NEXT GENERATION NUCLEAR PLANT & Identifier: & PLN-2498 & \\
METHODS RESEARCH AND & Revision: & 1 & \\
DEVELOPMENT TECHNICAL & Effective Date: & $9 / 25 / 08$ & Page: 58 of 188 \\
PROGRAM PLAN & & & \\
\hline
\end{tabular}

heterogeneity effect. It also suggested that additional development might be required for better accuracy of the double heterogeneity effect particularly when the effect is magnified by the presence of transuranics and as the kernel diameter becomes bigger. Future activities will focus on a complete assessment of the DRAGON code deficiencies and identification of the necessary modifications that would make the code attractive for prismatic VHTR applications. These would include evaluation of the double heterogeneity and resonance treatments for coated fuel particles, the best format for the crosssection data library (DRAGON does not come with its own library), transmutation and decay chains, modularity of the code for staged calculations, and code performance compared to that of higher fidelity models/codes (e.g., Monte Carlo simulations, see Figure 16). It is also intended to obtain better code documentation and descriptions of advanced models and capabilities not in the public domain (e.g., methods of characteristics solution, homogenization/de-homogenization, and parallel code version).

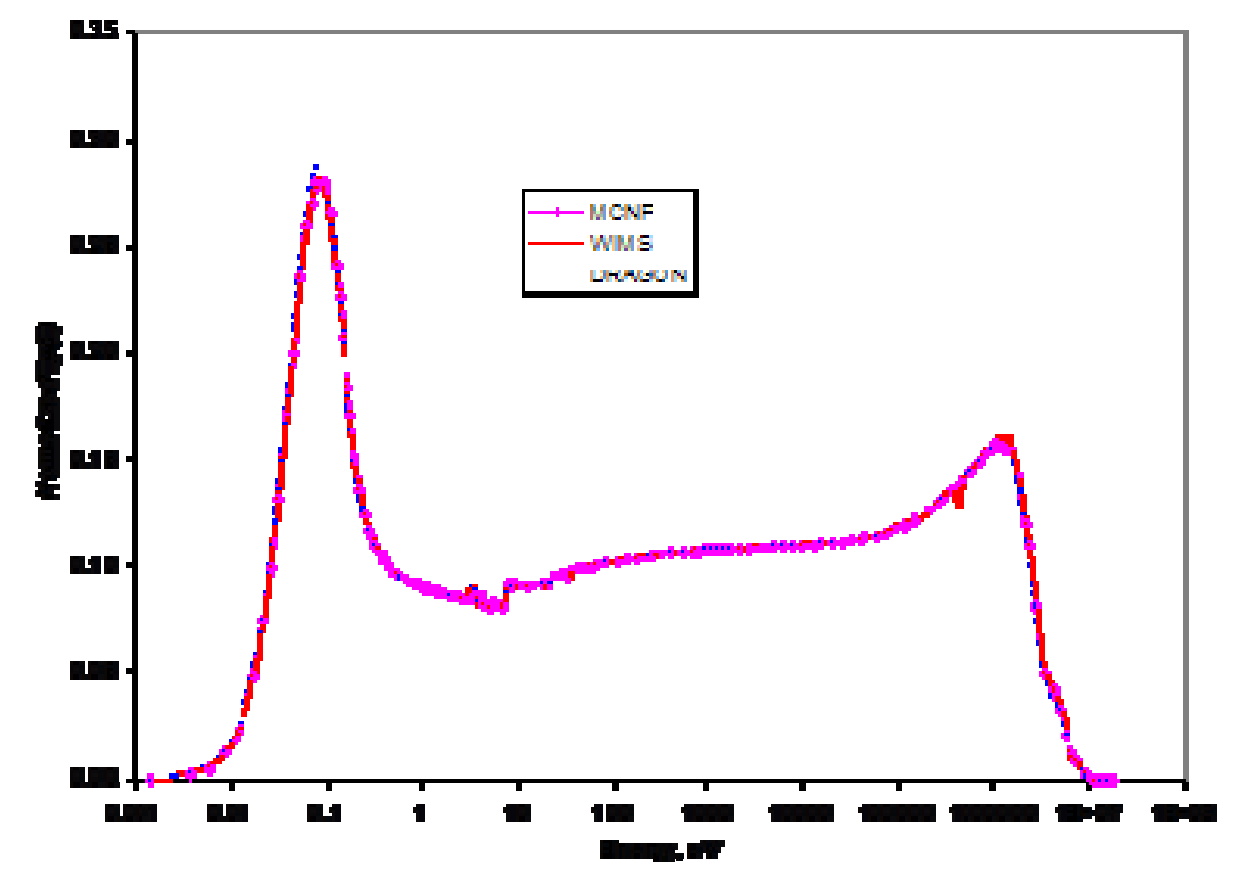

Figure 16. Comparison of VHTR spectra generated by various codes (ANL).

This effort will be coordinated with other ongoing and proposed projects. For example, there is already an I-NERI project underway and centered at the ANL to develop safety analysis codes with experimental validation for a prismatic VHTR. The collaborators include the University of Michigan and the Korea Advanced Institute of Science and Technology (KAIST). One of the tasks in this project is to use MCNP to develop cross-sections for DIF3D in place of a deterministic assembly code. This project complements the DRAGON effort and will provide an essential comparison of methods. While useful for principal cross sections, however, generation of cross sections with Monte Carlo calculations is not very practical for group scattering cross sections given the tremendous computational effort required. Other approaches will be investigated. A similar effort centered at INL will include the acquisition of a comprehensive methodology and related code patches to compute multigroup diffusion data using a modified version of the MCNP code in order to use it for benchmarking the other capabilities being developed based on HELIOS and COMBINE. 


\begin{tabular}{|c|lll|}
\hline NEXT GENERATION NUCLEAR PLANT & Identifier: & PLN-2498 \\
METHODS RESEARCH AND & Revision: & 1 & \\
DEVELOPMENT TECHNICAL & Effective Date: & $9 / 25 / 08$ & Page: 59 of 188 \\
PROGRAM PLAN & & & \\
\hline
\end{tabular}

To devise an appropriate functionalization scheme, the dependencies of cross sections of the NGNP fuel block on various state variables will be investigated using results of the DRAGON and WIMS8 lattice codes. It is noted that the planned code suite is not limited to the use of these two codes for crosssection generation. Other lattice codes can be plugged later into this code suite, once their performance for VHTR analyses has proven to be satisfactory.

A group-constant processing code will be written that automates the process of generating homogenized multigroup cross sections for the fuel, reflector, and control blocks as functions of state variables. Nodal equivalence theory parameters and directional diffusion coefficients will also be generated to account for the homogenization errors expected in the regions where significant material discontinuities exist. Examples of these regions are the core/reflector interface and control rod regions. A group constant functionalization scheme will also be developed and implemented in the group constant processing code. Both table lookup and polynomial fitting approaches will be considered.

A recent evaluation of the DRAGON code capability was completed in 2006 . The limitations that have been identified for the current version of the DRAGON code, for VHTR analysis, include the following details.

A typical prismatic block assembly has, by design, flat surfaces. The DRAGON code can model hexagonal cells (even with internal non-hexagonal structures), but it cannot model full hexagonal assemblies. Therefore, in order to model assemblies (for the generation of discontinuity factors) an approximate approach was used. The assembly is represented with a jagged boundary resulting from the juxtaposition of hexagonal cells. Furthermore, the evaluated version of DRAGON suffers from a lack of edits for surface fluxes and currents - the lack of a flat surface boundary in DRAGON calculation for a fuel block has necessitated the development of a temporary fix. In order to generate surface fluxes and currents required for generating nodal equivalence parameters (discontinuity factors) for the block surfaces, small circular regions are added to all peripheral hexagonal cells; note that pin-cell sized hexagons are used to compose a hexagonal block. The flux and current edits for these small cells are used to approximate the surface-average fluxes of the fuel block. A better representation of the surface parameters is needed in the future. The geometric limitation is now described in more detail; the evaluated version of DRAGON (Version 3.5) is able to model hexagonal cells containing circular pins, but it is not flexible enough to mix hexagonal and circular geometries in the assembly configuration. Thus, the fuel-element handling hole at a central position of the fuel block is approximated with 2-ring hexagonal cells (7 cells). Since the graphite density of the fuel-element handling hole is not different from that of normal fuel elements, this approximation would be valid. It is noted that in the DRAGON fullassembly model for the VHTR hexagonal block, the block is formed by a collection of pin-cell sized hexagons (see Figure 16 above). Each pin-cell contains the fuel compact surrounded by block graphite. When all the fuel and coolant-hole pin-cells are represented, the block graphite content is not totally accounted for, and therefore an extra ring of pin-cell sized hexagons is used to represent the remaining graphite. The number density of the graphite in these peripheral cells is modified to preserve the graphite content of the assembly block. Because of the pin-cell sized hexagons, the DRAGON assembly model has jagged boundaries, and not the flat boundaries of the hexagonal block. This jagged boundary model precludes the direct calculation of surface average fluxes for the generation of discontinuity factors. Although the modification of DRAGON for a flat boundary is an ultimate solution to the problem, the temporary fix described above has been used in the NGNP project.

1. No explicit geometry representation for large control rod channels. The current work around is to use pin-cell sized hexagonal cells to represent the control rod. To preserve the reactivity worth of the control rod, the control rod zone is divided into three sub-zones, and different nuclide number 


\begin{tabular}{|c|lll|}
\hline NEXT GENERATION NUCLEAR PLANT & Identifier: & PLN-2498 \\
METHODS RESEARCH AND & Revision: & 1 & \\
DEVELOPMENT TECHNICAL & Effective Date: & $9 / 25 / 08$ & Page: 60 of 188 \\
PROGRAM PLAN & & & \\
\hline
\end{tabular}

densities are assigned to each sub-zone. This current model, for which a reference solution (such as MCNP) is required, needs to be improved in the future.

2. Limited restart capability making branch-case calculations tedious.

3. No explicit treatment of fuel-particle double heterogeneity effect within the highest-fidelity EXCELT solution option.

4. Additional Validation and Verification $(V \& V)$ of the code capability. Preliminary V \& V of the DRAGON code has been performed. However, as the new capabilities are implemented, there is a need to re-evaluate the code results and document its performance in a topical report that would be part of the licensing basis for the codes.

5. Multi-assembly calculation capability for extended lattice calculations to generate surface discontinuity factors and nodal cross sections.

These items should be addressed in the future to improve solution accuracy and flexibility. Additionally, results for the fuel block suggest that more work is required to improve the DRAGON code accuracy for certain fuel double heterogeneity problems (cases containing enriched uranium fuel kernels with large diameter). This issue has to be addressed in the future. It has also been recommended that effort be devoted to the creation of a base crosssection library tailored for VHTR/NGNP analysis. Currently, base cross-section libraries developed generically for thermal reactor systems (mostly for water-cooled system) have been employed.

\subsection{Static Analysis for Evaluations of Criticality and Power Distribution (Core Simulation)}

The fundamental quantity in reactor physics analysis, which determines all other aspects of core behavior, is the neutron flux distribution. Extremely accurate calculations of the neutron flux, accounting for geometric details, can be made with Monte Carlo codes such as MCNP. However, Monte Carlo codes are still prohibitively expensive for use in repetitive design and tradeoff or scoping calculations or analysis to determine local reaction rate distributions or small reactivity effects. Nor can current coupled Monte-Carlo-depletion codes be applied to the pebble-bed reactor, because they do not account for fuel movement during operation. Deterministic codes offer much greater computational speed, at the cost of reduced geometric modeling capability. However, the natural geometric configurations of both pebblebed and prismatic VHTRs lend themselves to accurate modeling by deterministic codes (circular cylindrical geometry for the pebble-bed reactor and hexagonal geometry for the prismatic reactor) provided that the relevant physics is captured in the generation of few-group cross sections.

\subsubsection{General Considerations in Static Core Simulation with Diffusion and Transport Theory}

Nodal Diffusion: Earlier methods applied finite-difference techniques for spatial discretization. In the finite-difference approach, the governing equations (e.g., the neutron diffusion equation) are approximated as difference equations valid within very small mesh elements. Acceptable accuracy is obtained by making the mesh elements very small, so that the difference equations are close approximations of the original differential equations. Neutronics methods developed in the past for PBRs, such as the code V.S.O.P., rely on finite-difference methods. Modern neutronics tools are based on nodal methods, in which the governing equations are solved exactly (or with powerful analytical 


\begin{tabular}{|c|lll|}
\hline NEXT GENERATION NUCLEAR PLANT & Identifier: & PLN-2498 \\
METHODS RESEARCH AND & Revision: & 1 & \\
DEVELOPMENT TECHNICAL & Effective Date: & $9 / 25 / 08$ & Page: 61 of 188 \\
PROGRAM PLAN & & & \\
\hline
\end{tabular}

approximations such as expansion techniques) in spatial regions ("nodes") much larger than finitedifference mesh elements. Because of the much coarser spatial discretization, nodal methods are far more computationally efficient than finite-difference methods - roughly an order of magnitude faster in each spatial direction.

A new nodal technique for cylindrical geometry was developed with NERI funding and is being implemented in PEBBED. This NERI drew on support or cooperation from the Georgia Institute of Technology, Penn State University, the University of Arizona, and PBMR (Pty), Ltd. of South Africa. Because finite-difference formulations can be coded directly from the governing equations, the diffusion equation solver in PEBBED was originally written as a finite-difference code, so that the underlying algorithm could be tested quickly. But it was written in a modular form so that nodal methods could easily be inserted to replace the finite-difference solution. Work is under way to implement an analytical nodal solver in cylindrical coordinates developed at INL as part of an LDRD project and continued under the NERI program. The solver has been successfully demonstrated for one and two-dimensional (RZ) problems and in hybrid version as RZ-nodal theta-finite difference. Once fully implemented, the nodal (in all three directions) diffusion solver will yield a highly efficient and accurate code for all PBR calculations.

While PEBBED has been used for some basic equilibrium core design problems, it still required development of some key features that will allow it to be used extensively as a design and analysis tool for the NGNP. Some of the more important ones are:

- Complete implementation of the integrated cross-section generation module that accurately treats resonance effects of the doubly heterogeneous fuel, leakage, and temperature effects

- Implement the capability to model time-dependent fuel loading cases (non-equilibrium cores), including the accurate description of pebble flow based upon first principles

- Implement coupled neutronic and thermal-hydraulic transient capability that properly computes fuel and moderator temperatures

- Implement a gamma photon transport and adjoint (variational) computational capabilities.

PEBBED possesses an advanced optimization routine (based on genetic algorithms) that allows automated searches for optimal core designs and fuel loading patterns. Such methods have been applied to LWR codes for some time, but they have yet to be developed for the prismatic reactor. This will need to be addressed. For the prismatic reactor design, fuel blocks are proposed that may have compacts with differing enrichments, packing fractions, and burnable poison concentrations. The block-refueling pattern may be strictly radial or may have an axial shuffling component as well. Core optimization and fuel loading must be automated to some extent to produce viable cores within practical time limits. There are a number of advanced optimization approaches, including genetic algorithms, simulated annealing, neural networks, Tabu search, and others. One or more of these will be explored and implemented in conjunction with DIF3D/REBUS-3 and the prismtic version of the PEBBED suite at INL.

The REBUS-3/DIF3D depletion code system would be further developed for the analysis of prismatic-type VHTR designs. The code system is capable of multigroup flux and depletion calculations in hexagonal-Z geometry. The DIF3D module, which contains both nodal diffusion and transport theory capabilities, is used as the neutron flux solver in the code system. The code system would be adapted to prismatic VHTR problems with limited effort. However, it requires additional work to implement a thermal feedback model and a tabulation scheme of nodal cross sections versus depletion and temperatures. To capture the large variations in temperatures, DIF3D will need to be coupled to a thermal 


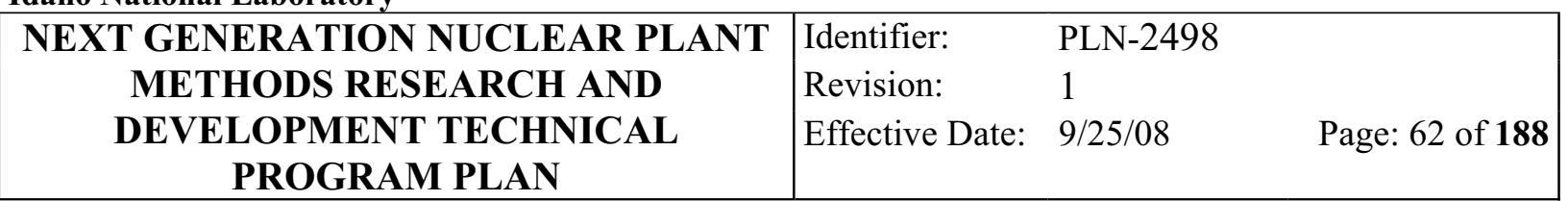

fluid module just as PEBBED has been coupled to THERMIX. Additionally, a lumped fission product model is needed to make the microscopic depletion scheme used in REBUS-3 (and also in PEBBED) practical for high-burnup criticality calculations. The code would also have to be further modified to employ the nodal equivalence parameters that could be used for improving solution accuracy (beyond the modification already completed). These parameters are useful for reducing the errors arising from the flux gradients in the transition regions around the core and reflector interfaces and the heterogeneity effects arising for the insertion of strong absorbers in fuel and reflector regions.

Neutron Transport Techniques and Applications: For some core physics issues, diffusion methods are not appropriate, and detailed neutron transport methods are required. Often, it suffices to use transport methods on a local scale, to incorporate transport effects into diffusion-theory parameters such as cross sections, as discussed in the previous section. For example, in the pebble-bed reactor, control rods are required to shut the reactor down rapidly on demand and keep it subcritical at low temperatures. The pebble-bed reactor also contains a large gas plenum above the core through which pebbles are dropped. Diffusion theory alone cannot accurately predict neutron transport in these regions, so some sort of transport calculation or correction is necessary. Nor does diffusion theory solve the gamma transport problem and thus the actual heat deposition distribution differs from what it can compute. Various wholecore transport methods and codes are being developed that can address these problems. Partial-core models can also be used to quantify the error resulting from the diffusion approximation. For the prismatic core analysis, DIF3D contains a variational transport solver that can properly treat regions in which diffusion theory is not valid. A NERI project, led by F. Rahnema of the Georgia Institute of Technology in conjunction with INL, is investigating novel neutron transport techniques that can be used to accurately treat gas plenums and control rod regions in the pebble-bed reactor. In addition to code and model development activities, reactor physics workshops and meetings on this item will be conducted throughout the life of the NGNP project.

Monte Carlo techniques are free from all these NGNP modeling issues if fuel particles are modeled explicitly in the core calculation. However, this detailed modeling is currently unattractive because of the tremendous problem size and because a very large number of neutron histories is required to resolve fuelelement power distribution and small reactivity effects. As a practical matter, the necessary calculations are beyond the current capabilities of even the most sophisticated computers. Furthermore, several important phenomena such as thermal feedback at power generating conditions, flux uncertainty propagation in the depletion calculation, and fission product buildup are not properly addressed in these tools at the present time. To do so with the Monte Carlo technique would increase the computational requirements even further. In contrast, deterministic three-dimensional whole-core transport calculation provides a possibility of resolving all these problems as long as the proper fuel modeling and thermal feedback capability is incorporated in the underlying effective cross-section data.

The ATTILA code solves the $S_{n}$ equations on an unstructured finite element mesh. It is compatible with widely available CAD software for user-friendly core modeling and features a very efficient Discontinuous Galerkin acceleration scheme for the source iteration. The ATTILA code was used to model the INL's Advanced Test Reactor as part of several projects. Nonetheless, full-core transport modeling will still require tremendous computational power and is not practical for scoping or design optimization calculations. Rather, such techniques (Monte Carlo or deterministic) will be useful for benchmarking activities once a design has been rendered using the other approaches described above.

Transport techniques may be applied successfully to core simulations in local or reduced dimensional (2-D or 1-D) computations. High temperature reactors have somewhat different neutronic properties than standard LWRs, specifically, a higher thermal flux component with the peak shifted to higher energy, a 


\begin{tabular}{|c|lll|}
\hline NEXT GENERATION NUCLEAR PLANT & Identifier: & PLN-2498 \\
METHODS RESEARCH AND & Revision: & 1 & \\
DEVELOPMENT TECHNICAL & Effective Date: & $9 / 25 / 08$ & Page: 63 of 188 \\
PROGRAM PLAN & & & \\
\hline
\end{tabular}

longer mean free path compared to core dimensions, a more complex fuel geometry, and a fuel cycle with two to three times the burnup. The longer mean free path means that there is a significant amount of leakage between blocks or zones that would not be fully captured using typical LWR analysis methods. V.S.O.P. and PEBBED account for leakage by using buckling terms, provided by the core simulation, in the spectrum calculation. This approach may have a limited accuracy. A better technique would be to model the radial dimension fully with a 1-dimensional transport calculation and homogenize the crosssections over space and energy within each major region. This is being explored with PEBBEDCOMBINE. The same approach is being considered at INL for the generation of zonal cross sections for the annular VHTR designs, which also are characterized by strong radial neutron leakages and spectral interchange between radial zones. Other techniques may prove useful as well.

Detailed power deposition calculations, taking into account transport of gamma rays, are necessary for thermal calculations, coolant flow allocation and orifice design, and the simulation of irradiation behavior of the graphite matrix of the fuel and reflectors. These calculations require that gamma production and transport in the core be accurately calculated. The DIF3D/GAMSOR calculational path will be evaluated and modified for use in NGNP analysis for the prismatic core. For the pebble-bed core, an alternate code will be identified.

\subsubsection{PBR-Specific Analysis}

Special considerations for pebble-bed reactors. Until recently, design analysis methods for PBRs have been several generations behind the state of the art for LWR design and analysis. For the past seven years, INL has been engaged in the development of analysis methods for high-temperature gas reactors, with a special emphasis on the pebble-bed reactor and the PEBBED fuel management code. A sample PEBBED graphical neutron flux output is shown in Figure 17. Using genetic algorithms developed to work in conjunction with PEBBED, INL optimized design parameters to achieve a passively safe pebblebed reactor design of $600 \mathrm{MWt}$, a goal that had not previously been attained. Using PEBBED, INL was also able to propose design enhancements to PBR fuel that increased safety during a potential water ingress accident and improved fuel economy and utilization. These design improvements were attained as incidental results of the verification of the new methods and the testing of their capabilities.

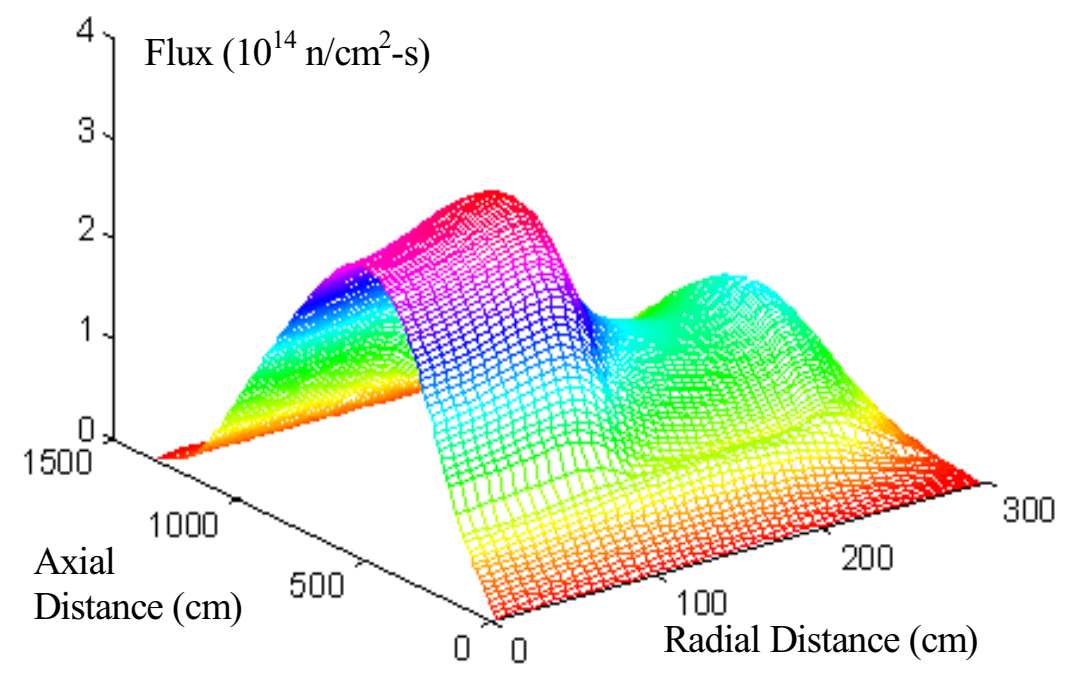

Figure 17. Thermal neutron flux profile in the NGNP $600 \mathrm{MWt}$ reactor calculated by PEBBED. 


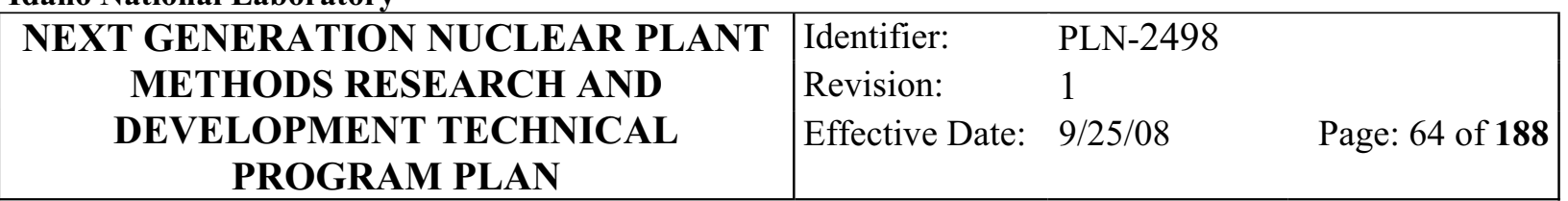

One of the licensing issues for PBRs is the perception that the semi-stochastic nature of the pebble distribution admits the possibility of collection of relatively reactive pebbles in regions of high neutron flux, so that the local power density could become excessive either in normal operation or in accident scenarios. Some estimates of the consequences associated with this phenomenon have been performed at INL. Various pebble flow and stochastic clumping scenarios were modeled that resulted in abnormally high concentrations of fresh fuel. The probability of such configurations occurring as a result of stochastic variation is many orders of magnitude lower than the typical beyond-design-basis events normally considered in high-temperature gas reactor analysis. Yet the nominal and accident fuel temperatures attained during these events was shown to be only somewhat higher than normal and still lower than those required to induce significant fuel failure. However, in light of the pebble temperature measurements made in the AVR, some of which were much higher than expected, a more rigorous analysis is warranted and is possible with advanced Discrete Element techniques and other new modeling tools. Such an effort is underway at INL using a molecular dynamics approach. The modeling techniques should be employed to characterize and bound the stochastic component of pebble movement and feed these probabilities to a reasonably conservative core safety analysis. These tools can also be used to develop flow models for pebbles in the discharge and entry regions and to examine pebble-packing issues.

PEBBED is a combined diffusion/depletion code conceived to solve self-consistently for the neutron flux and the burnup distribution in a pebble-bed reactor with circulating pebbles. As noted above, it was originally written with a finite-difference diffusion solver, but nodal diffusion modules have been installed in the code and are currently undergoing checkout and debugging. INL personnel have developed an analytical nodal solver using a "moments-stepping" method that allows for variable cross sections within nodes. Such advances in burnup calculations will be explored as a complement to the development of the nodal diffusion solver.

Pre-asymptotic core analysis in the pebble-bed reactor. Pebble bed reactors that use a mix of fresh fuel and re-circulated fuel gradually approach an equilibrium fuel burnup distribution pattern (or nuclides number density pattern). This pattern is reached after varying intervals of time, depending upon the recirculation scheme, the fuel design (e.g., enrichment, fuel loading per pebble etc.), and the burnup cutoff (at which pebbles are discarded instead of being further recirculated. The equilibrium state is also termed the "asymptotic" state and the corresponding loading pattern the "asymptotic" loading pattern. Before the core reaches this asymptotic state, it is said to be in a pre-asymptotic state. The PEBBED code obtains the asymptotic distributions of neutron flux and burnup directly, without following the timedependent distributions in the run-in period. This property of the code permits very rapid solution. However, a typical pebble-bed reactor may take as many as 3 years to achieve an asymptotic state. A theoretical formulation for pre-asymptotic core analysis is under way at INL. It will ultimately lead to the coding of a time-dependent solution of the coupled pebble-flow/burnup problem.

Non-axial pebble flow in the pebble-bed reactor. The flow of pebbles in a pebble-bed reactor is not strictly axial, particularly near the discharge tubes (see Figure 18). While the neutronic importance in this region is minimal, a method and code must be developed that link together depletion zones along the true flow path of pebbles even for flow lines that are not strictly axial. In this development the axial flow of pebbles is modeled, as in the previous case. In addition, the radial drift of pebbles is also captured. Effective pebble flow characteristics are developed and used to link computational coarse nodes systematically. Experiments and some computations have been performed that confirm and characterize the strong deterministic (streamlined) component of pebble flow. A computational approach to pebble flow using a particle dynamics approach has been initiated at INL. Promising results were generated since 2005, with advanced applications, such as earthquake modeling, demonstrated in 2006 (Cogliati and Ougouag 2006). Similar efforts were underway at various universities in the last 6 years. Results from 


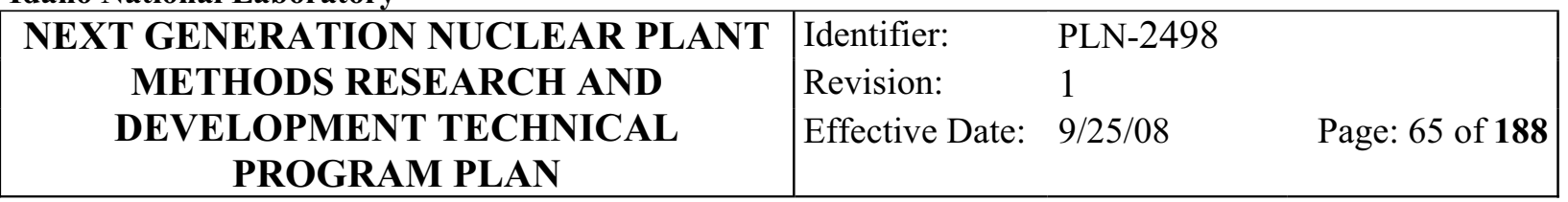

these efforts will be used to construct the flow lines over which the depletion equations are solved in PEBBED.

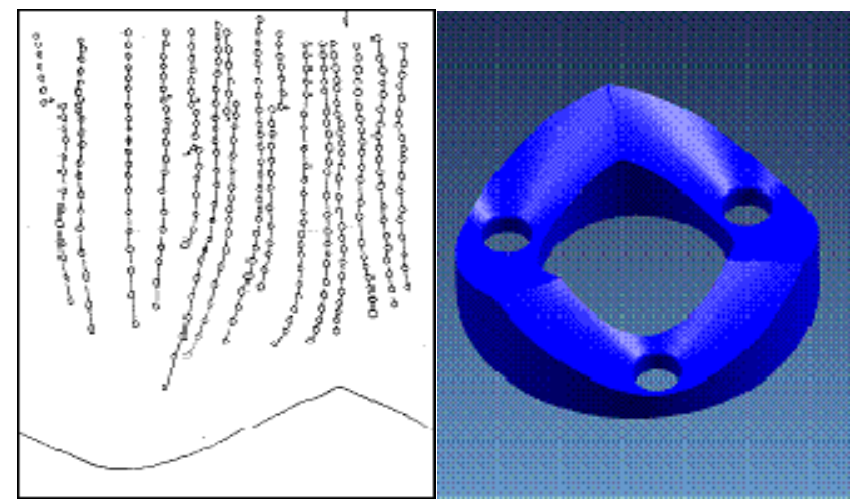

Figure 18. Pebble flow lines (left) and sketch of the defueling cones near the bottom of the PBMR core (right).

Variable Pebble Packing and Slumping: Experiments relevant to the PBR have shown that the average packing fraction of pebbles in the vat is expected to be about $61 \%$, but that in the presence of shaking (such as may occur during an earthquake), the packing fraction may increase to as much as $64 \%$. Calculations have been done to show that an increase of packing fraction from $61 \%$ to $64 \%$ may cause significant "reactivity transients" (i.e., power surges) in a PBR. A computer model that couples the mechanical slumping of the pebble bed to the neutronics and the thermal-hydraulics is necessary to the comprehensive modeling of the earthquake effects. Such a computer code and related models are under development at INL.

Furthermore, the packing is known not to be uniform. The packing fraction is exactly zero at solid walls and approaches an asymptotic value through a series of spatial oscillations over a distance of several pebble diameters. In a PBR, such a distance may be a significant portion (e.g., 20\% or more) of the vat radius. If the vat is surrounded by a neutron reflector, as it probably will be, these fluctuations occur in a region of high-thermal neutron flux, so that the fluctuations will have exaggerated importance. The modeling effort underway at INL will be able to address many of these issues.

\subsubsection{Prismatic-Specific Analysis}

Special considerations for prismatic reactors. In 2003, INL developed "point designs" for pebblebed and prismatic-fuel versions of the NGNP (MacDonald et al. 2003). The objective of the point design project was to develop a reactor specification with a mixed mean coolant core outlet temperature of $1000^{\circ} \mathrm{C}$, passive safety, and about $600 \mathrm{MWt}$ of power. Sensitivity studies for various block-fuel parameters were performed at ANL and INL to address design issues critical to this objective and to provide data for developing a fuel specification. For modeling the block-type NGNP with great geometric detail, the Monte Carlo code MCNP has proven itself to be a very powerful tool; in conjunction with the ORIGEN depletion code with a coupling code such as MOCUP, it can follow a block-type core through its fuel cycle and produce accurate burnup and isotopic buildup data in each fuel block. Since MCNP requires long calculation times to produce good statistics, it is not a practical tool for performing large numbers of calculations in design studies, generating detailed core power distribution, or analyzing the effects of small perturbations. For some scoping studies and sensitivity analyses, the ANL codes DIF3D and REBUS-3 have been successfully applied in conjunction with WIMS8 and DRAGON lattice codes 


\begin{tabular}{|c|lll|}
\hline NEXT GENERATION NUCLEAR PLANT & Identifier: & PLN-2498 & \\
METHODS RESEARCH AND & Revision: & 1 & \\
DEVELOPMENT TECHNICAL & Effective Date: & $9 / 25 / 08$ & Page: 66 of 188 \\
PROGRAM PLAN & & & \\
\hline
\end{tabular}

for model cross-section generation. These lattice codes have models for treating the double heterogeneity effect resulting from the use of coated fuel particles in the graphite matrix. However, additional developmental work needs to be done to improve computational models and user friendliness. More recently, computational techniques and advances in parallel computing have made feasible more detailed 3D deterministic transport calculations for some applications including for accurate gamma transport and deep penetration/shielding. DeCART, ATTILA, and EVENT are codes that will be explored for use in the project. Other transport codes that prove relevant to VHTR systems will be evaluated.

It is noteworthy that the DeCART code performs whole-core transport calculation in fine group level for heterogeneous geometries, and thus it avoids the cross-section homogenization and condensation steps. ATTILA and EVENT also can be run with very high energy resolution, but they still require the more traditional multigroup cross-section processing. Under an LDRD project, the ANL is also developing the UNIC code that will use a large number of groups $(10,000)$ and explicit geometry details for direct, whole-core calculations. While this approach will increase solution accuracy, it will however require huge computing resources. The coupling of the second order form of the transport equation with the first order one will allow to overcome the problem of low density regions in this formulation. The integration of the code with a mesh generating capability is under way, and computation of complex geometries started in early 2007. Further development and validation and verification of the capability are planned.

Full-core transport modeling with these codes will still require tremendous computational power and is not practical for scoping or design optimization calculations. Rather, such techniques (Monte Carlo or deterministic) will be useful for benchmarking activities once a design has been rendered using the other approaches described above. With continued advances in computer power and implementation of innovative numerical solution methods, these techniques may in the future provide a practical, highfidelity capability for routine use in design and analysis. As these codes become relatively efficient for VHTR core analysis, a selection of the most suitable one will be made and included in the VHTR design and analysis calculation path.

Work has been initiated at ANL to implement a code suite based on the DIF3D/REBUS-3 code system. An ANL report issued in September 2006 (Lee et al., REBUS-3/DIF3D) contains a documentation of this effort. The report identified some REBUS-3/DIF3D code features that require further development or upgrade for VHTR analysis. The three pending tasks are:

1. The accuracy of the current thermal feedback model based on the SAS code and used in the ANL code suite needs to be verified in the future using reference calculations with coupled neutronic and thermal-fluid models. Additionally, the thermal feedback routine should be extended to equilibriumcycle depletion calculations; it currently works for the traditional non-equilibrium cycle depletion calculations.

2. Advanced nodal diffusion methods typically employ nodal equivalence parameters (discontinuity factors) to reduce homogenization errors arising from core heterogeneity (different rodded and unrodded fuel and reflector regions, and interfaces between the regions). Surface-dependent discontinuity factors are particularly very useful to take into account geometric asymmetry in the nodal approach and thus must be provided. The need for surface-dependent discontinuity factors in nodal calculations necessitated the modification of several routines in the DIF3D-nodal Hex-Z version of the code (DIF3D-nodal). It was originally thought that using surface-dependent discontinuity factors in the DIF3D-nodal option would give good accuracy for all core configurations. This has not been the case for rodded configurations, due to the relatively poor transverse leakage approximation made for the nodal option (particularly when a large hexagonal pitch is used in the code). Generally, REBUS-3/DIF3D results for the core multiplication factor and power distribution 


\begin{tabular}{|c|lll|}
\hline NEXT GENERATION NUCLEAR PLANT & Identifier: & PLN-2498 \\
METHODS RESEARCH AND & Revision: & 1 & \\
DEVELOPMENT TECHNICAL & Effective Date: & $9 / 25 / 08$ & Page: 67 of 188 \\
PROGRAM PLAN & & & \\
\hline
\end{tabular}

were found to be in good agreement with MCNP results particularly when discontinuity factors are applied. It was also shown that the DIF3D-VARIANT option provides a better spatial solution in its diffusion approximation. In addition, it was observed that control rod worths could be estimated within an acceptable range compared to MCNP results. However, the core power tilt (particularly in the rodded zones) needs to be improved by introducing surface-dependent discontinuity factors. The improved performance with surface-dependent discontinuity factors was shown at the mini-core level. It was therefore recommended that a routine for surface-dependent discontinuity factors be provided for the VARIANT option of DIF3D (DIF3D-VARIANT) or the spatial approximation of the nodal option (DIF3D-nodal) be improved in the future. Most of the developments necessary to these modifications were carried out in FY 2008 using NGNP funding for the generation of a depletion benchmark and its solution. Great improvements have thus been demonstrated; however, full fidelity still remains elusive and additional work to resolve the remaining issues should be carried out.

3. Pin powers distributions are reconstructed by using imbedded local calculations or by superposition of global and local power distributions, which are respectively obtained from nodal and lattice calculations. Pin power factors generated from the lattice code (DRAGON) need to be implemented in DIF3D, by folding their values with the global information provided by the whole-core nodal calculations.

\subsection{Kinetics, Thermal Module Coupling, and Feedback}

Three-dimensional spatial kinetics capabilities have been under development for more than 20 years. Practical tools now exist and include the public versions of NESTLE, PARCS, VARIANT-K, and DIF3DK. These high-fidelity kinetics methods are important for core transients involving significant variations of the flux shape, but they have not been systematically applied to graphite-moderated, heliumcooled reactors. In the future, integrated thermal-hydraulics and neutronics methods should be extended to enable modeling of a wider range of transients pertinent to the VHTR. Required advances include increasing the efficiency of the coupling approaches and improving the representation of cross section variations.

For the pebble-bed reactor, the PEBBED solver has already been coupled to the THERMIX code for thermal-hydraulics feedback. The coupled code is used for the computation of the flux and power distribution, taking into account temperature feedback and depletion (or, equivalently, the fuel loading pattern). In addition to the PEBBED code a standalone kinetic module based on the nodal solver within PEBBED has been written. This kinetics code has also been coupled to THERMIX as well as to RELAP5-3D, thus providing a unique and powerful capability for the analysis of reactor dynamics that has been demonstrated on challenging benchmarks (OECD NEA PBMR400 Benchmarks). Typical PBMR transient results are shown in Figure 19.

Kinetics parameters such as the delayed neutron fraction will also have to be generated for the newly completed kinetics code. These are derived from the nuclear data files, and thus corresponding modules must be included in the lattices codes used for generating group constants (cross sections, etc.) for the whole-core analysis codes. Additionally, kinetics parameters are required for core dynamics codes that use the point kinetics approximation. These are generally derived using inner product calculations that require flux and adjoint distributions obtained from the whole-core calculations. DIF3D possesses adjoint capability. The finite difference version of the PEBBED solver also possesses such a capability; however, the latter is not yet fully tested and its testing should be carried out in FY 2009. The analytic nodal equations of PEBBED will be modified to allow for adjoint solution, and the solver in PEBBED will be upgraded. 


\begin{tabular}{|c|lll|}
\hline NEXT GENERATION NUCLEAR PLANT & Identifier: & PLN-2498 & \\
METHODS RESEARCH AND & Revision: & 1 & \\
DEVELOPMENT TECHNICAL & Effective Date: & $9 / 25 / 08$ & Page: 68 of 188 \\
PROGRAM PLAN & & & \\
\hline
\end{tabular}

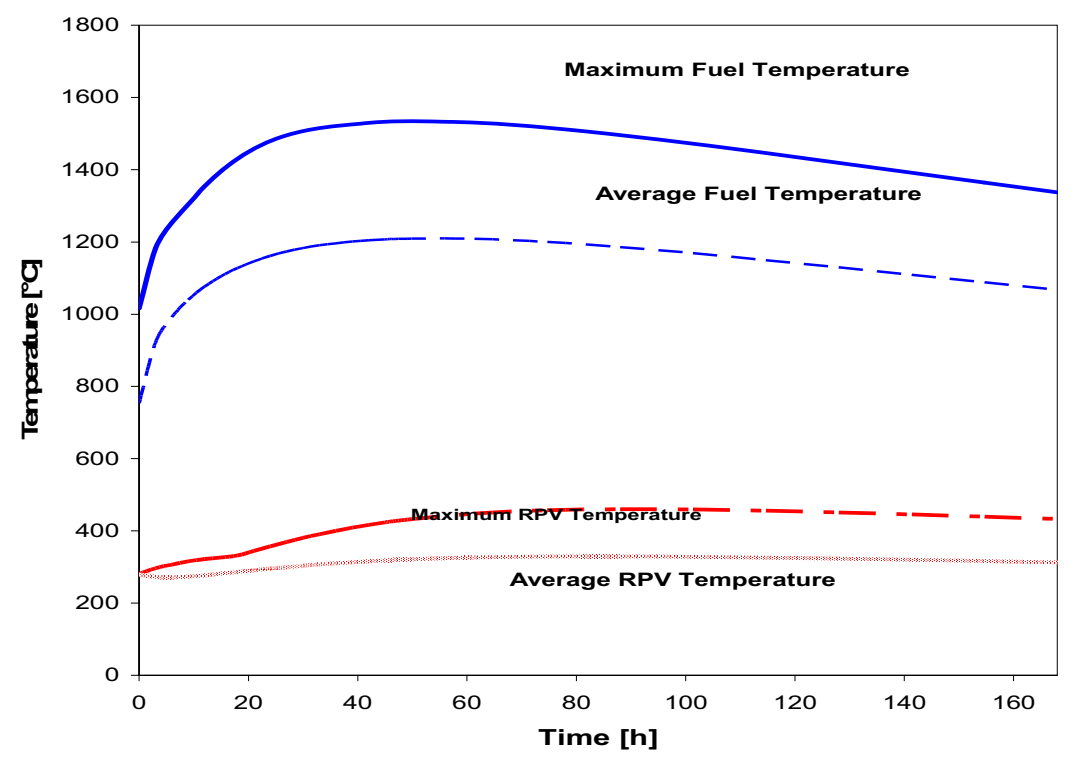

Figure 19. Temperature trajectory during a DLOFC event in the PBMR.

The use of THERMIX as the thermal-hydraulic code embedded within PEBBED is considered temporary as the version of THERMIX available at INL is severely limited for transient applications and in its lack of fidelity, especially with regard to feedback at the TRISO particle level. Furthermore, although THERMIX is a standard for PBR applications, it is still limited to two dimensions (R-Z), and thus cannot simulate 3-D transients such as single rod ejections. Ultimately, for incorporation within PEBBED, a replacement code will have to be acquired or written. Furthermore, the THERMIX module within the CYNOD code will have to be replaced by such a more modern code. For dynamic and balanceof-plant applications, the recently completed coupled RELAP5-3D-CYNOD code will be the tool of choice. However, this new coupled code must first be extensively benchmarked and subjected to V\&V. This will allow full systems and safety analyses of the PBR.

Perform quality assessment on thermal-fluids code: Thermal-fluids calculations are an important part of the safety analysis of the VHTR. For example, they can be used to estimate the temperature of the pressure vessel during a transient, thus providing essential information on the continued viability of the vessel following a transient (see Figure 19). Validation of the appropriate thermal-fluids tools is required for their use in such calculations. For PBRs, PEBBED possesses simple thermal-fluids models for estimating core temperatures. While these allow for rapid scoping and design optimization, they are unlikely to yield accurate thermal-fluids data. Assessment of various sophisticated thermal-fluids codes and comparison to PEBBED results are required to identify needed improvements in the PEBBED thermal models. For prismatic reactors, flow through the core is confined to well-defined coolant channels and gaps between fuel blocks (in which bypass flow occurs); thus, thermal-fluids in prismatic reactors can be modeled more straightforwardly than in PBRs. However, assessment of candidate thermal-fluids codes or models is also required for the selection of the best ones for prismatic reactor analysis. The detailed modeling of by-pass flow may provide plausible explanations for the German meltwire experimental results. 


\begin{tabular}{|c|lll|}
\hline NEXT GENERATION NUCLEAR PLANT & Identifier: & PLN-2498 \\
METHODS RESEARCH AND & Revision: & 1 & \\
DEVELOPMENT TECHNICAL & Effective Date: & $9 / 25 / 08$ & Page: 69 of 188 \\
PROGRAM PLAN & & & \\
\hline
\end{tabular}

There are a number of candidate thermal-fluids codes for VHTR applications. One of these is the INL's RELAP5/SCDAP suite of codes, and another is the INL's version of MELCOR. For several years, INL has been using MELCOR for PBR safety analysis, because it is able to model transport of the dust released by inter-pebble contact; the INL's version can also treat air and water ingress and helium coolant flow. However, these capabilities have recently been added to RELAP5/SCDAP, so it is not clear which of these codes is now better suited overall to model PBR safety problems. A third code is ORNL's GRSAC code, which was specifically written for gas-cooled reactors. A comparison will be made, and the chosen code will be validated for VHTR design applications. It is possible that different codes may be preferred for PBR and prismatic reactor analysis.

In 2005, the THERMIX two-dimensional gas reactor thermal-fluids code was coupled to PEBBED. THERMIX is widely used for PBR thermal analysis. It possesses a number of correlations and material properties specific to this reactor type. THERMIX provides improved steady-state thermal-fluid conditions and depressurized loss-of-coolant (DLOFC) transients. Benchmarking calculations have been completed and have demonstrated both the effectiveness and the limitations of this tool. The overall conclusion is that although it performs reasonably well in azimuthally symmetric configurations, it is a poor choice for the modeling of asymmetric transients.

Steady state thermal hydraulic calculations correctly assume a close coupling of fuel and moderator temperatures. This assumption is not valid in sharp transients during which the kernel temperature can rise dramatically and independently of the surrounding graphite. Whichever transient codes are used for either prismatic or pebble-bed analysis, a proper separation of fuel and moderator temperature effects must be implemented.

Nodal diffusion and transport kinetics capabilities have been developed for the DIF3D code in the past. These capabilities have been successfully applied for transient analysis of thermal reactor systems (e.g., NPR-HWR, RMBK, VVER, and LWR) by integrating them in a system analysis code, SASSYS. Initial estimation indicated that a multigroup analysis (about 20 groups) is required to represent accurately the reactivity effect of spectral change. The multigroup capability of DIF3D would be attractive for integration with a system code, such as RELAP5/ATHENA, that can be utilized for the analysis of the NGNP. Eventually, it can be upgraded with the new kinetics treatment described above. The RELAP code package contains the NESTLE kinetics module, which is currently limited to six energy groups. For consistency with the core static calculation approach, the NESTLE module would be replaced with the DIF3D kinetics module (nodal diffusion and/or transport) that allows multigroup calculations. Different approaches for coupling the DIF3D and RELAP codes will be investigated in order to produce an efficient integrated code package. Temporal coupling schemes (explicit or implicit or mixed) would also be investigated. This integrated code package would contain both the core flux calculation kernel and the cross-section fitting and interpolation routines developed for the REBUS-3/DIF3D code system under the NGNP activities. For a given core condition, including control rod axial positions, cross sections will be calculated and the flux distribution calculation would be performed. The core power distribution would be derived using the flux distribution and cross sections. This power distribution would be passed to the RELAP computation module and used in the evaluation of the thermal-hydraulic state of the reactor. The state data (e.g., densities and temperatures) would be passed to the neutronics module for the calculation of cross sections for the next set of flux calculations. This exchange of data between the neutronics and thermal-hydraulics modules will be performed for the duration of a given transient. 


\begin{tabular}{|c|lll|}
\hline NEXT GENERATION NUCLEAR PLANT & Identifier: & PLN-2498 \\
METHODS RESEARCH AND & Revision: & 1 & \\
DEVELOPMENT TECHNICAL & Effective Date: & $9 / 25 / 08$ & Page: 70 of 188 \\
PROGRAM PLAN & & & \\
\hline
\end{tabular}

\subsection{Material-Neutronics Interface}

Atomic displacements (i.e., radiation damage) are generally accepted as the underlying causes for many macroscopic manifestations (radiation effects) in materials that are subjected to irradiation by energetic neutrons. The estimation on the number of displacements caused in a given neutron field requires the knowledge of the displacement threshold energy (i.e., the lowest amount of energy that would cause a displacement if imparted to an atom in a solid). The displacement threshold energy $\left(\mathrm{E}_{\mathrm{d}}\right)$ depends on the temperature of the material, yet most experimental determinations of $E_{d}$ are carried out at temperatures close to room temperature, while models also assume a low temperature (often essentially 0 $\mathrm{K})$. For most applications, this is acceptable. However, for applications related to the VHTR reactor, the values of $E_{d}$ determined at $0 \mathrm{~K}$ or at room temperature may be inadequate. This project has acquired data for displacement energy threshold for materials of interest to the NGNP and has sponsored the development of a capability to obtain such data from first principles. Continuing related work has been funded directly by a NERI project at NC State University. Currently models and methods are under development at NCSU to assess damage to these materials and to assess the potential for annealing out at accident temperatures $\left(1200-1500^{\circ} \mathrm{C}\right)$. Annealing is essentially an exothermic process in which the energy stored in the atomic displacement is released as thermal energy. In graphite this may also result in a significant change in the scattering properties, thus causing a reactivity insertion. The full safety consequences of high-temperature annealing of VHTR core materials will be evaluated using state-of-theart molecular dynamics and Monte Carlo simulations.

Develop improved methods for calculating material damage from irradiation: The primary mechanism for material damage by neutron irradiation is atomic displacement (see Figure 20). The estimation of the number of displacements in a given neutron field requires knowledge of the displacement threshold energy $\left(E_{d}\right)$ (i.e., the lowest amount of energy that could cause an irreversible displacement to a non-equivalent lattice location if imparted to an atom in a solid). (A non-equivalent lattice location is a location such as an interstitial or a location corresponding to a different energy state [e.g., more or less stored potential energy].) In general, $E_{d}$ is expected to depend on the direction of motion in a crystal. This is due to the fact that $E_{d}$ may be viewed to represent the minimum energy needed to cross the potential barrier that surrounds an atom. However, because of the nature of crystallographic structure, the potential barrier may not be isotropic, which introduces variations in the threshold energy needed to overcome it. Furthermore, graphite-related experiments did show that $E_{d}$ in graphite depends on direction. In addition, computational simulations for $\mathrm{SiC}$ revealed a similar dependence for $\mathrm{E}_{\mathrm{d}}$ on crystallographic direction. On the other hand, the possibility does exist for $E_{d}$ to depend as well on the temperature of the material. In fact, experimental evidence for graphite shows such dependence. Nevertheless, most experimental determinations of $E_{d}$ are conducted at temperatures close to room temperature, whereas models also assume a low temperature (often essentially $0 \mathrm{~K}$ ). For most applications, this is acceptable. However, for applications related to VHTRs, the values of $\mathrm{E}_{\mathrm{d}}$ determined at $0 \mathrm{~K}$ or at room temperature may not be adequate. In this project, the values of $\mathrm{E}_{\mathrm{d}}$ for graphite and $\mathrm{SiC}$ have been determined as functions of direction and at temperatures representative of those of the structural materials and of the fuel in the VHTR. 


\begin{tabular}{|c|lll|}
\hline NEXT GENERATION NUCLEAR PLANT & Identifier: & PLN-2498 \\
METHODS RESEARCH AND & Revision: & 1 & \\
DEVELOPMENT TECHNICAL & Effective Date: & $9 / 25 / 08$ & Page: 71 of 188 \\
PROGRAM PLAN & & & \\
\hline
\end{tabular}
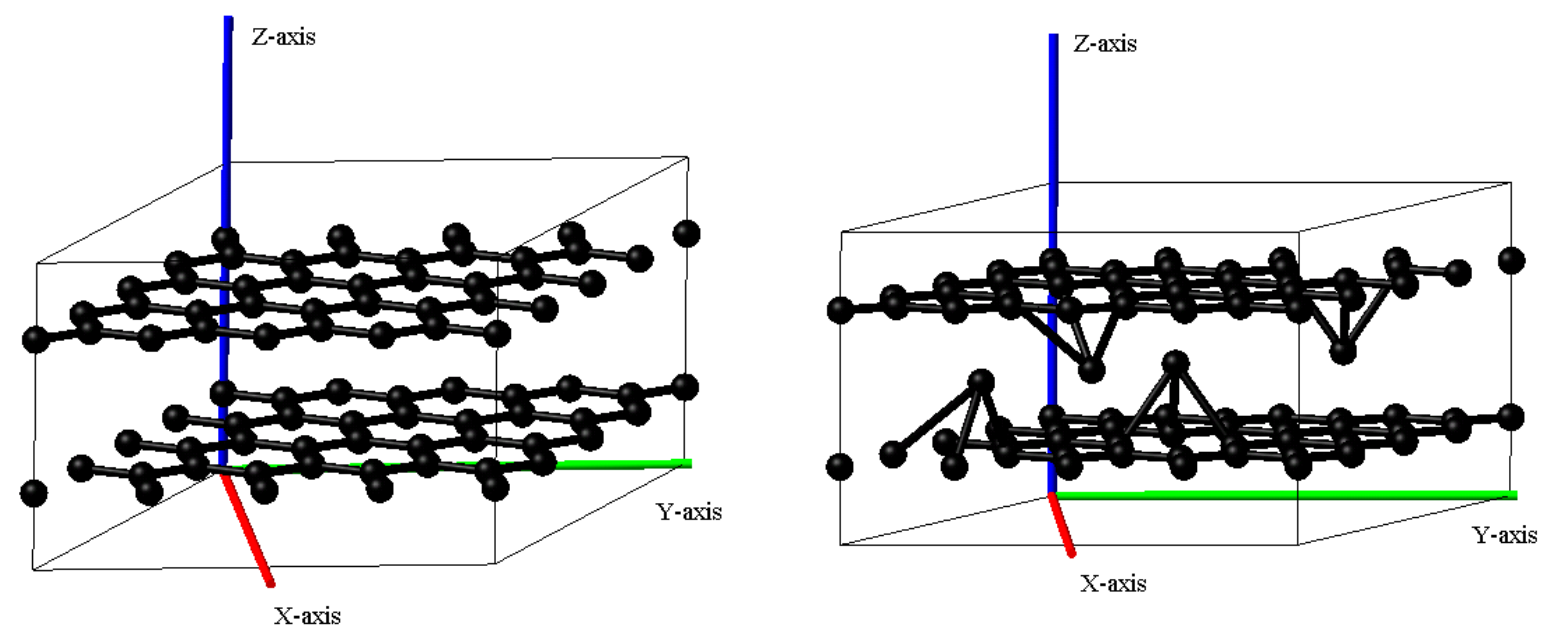

Figure 20. Ab initio (first principles) modeling of imperfections in a graphite crystalline structure.

The threshold energy $E_{d}$ for this displacement depends strongly on temperature, yet methods to account for this dependence have only now been developed. INL has recently developed a state-of-the-art method to estimate displacement damage cross sections in many materials of interest in the Generation IV program. Combined with the improved estimates for $E_{d}$, these data provide a comprehensive capability to estimate total number of displacements in a given neutron field. Work is continuing at NCSU to determine the damage configuration and their properties of importance to neutronics, such as phonon scattering and phonon spectra. The latter have a direct influence on the thermal conductivity and thermal neutron scattering of these materials. Another important artifact is the annealing of the radiation damage in high-temperature materials by the effects of heating and by further irradiation. A second goal of this task is to account for these annealing processes in the INL material damage model. This task is very complex and brings into play many areas of physics and computational science. For example, it requires a thorough understanding of the inter-atomic potentials in the solids under consideration and the dynamics of collisions in these materials. The proper modeling of the annealing will require the incorporation of thermal motion and its impact on effective average inter-atomic potentials. Also, to be incorporated is the modeling of collisions and atomic rearrangements, taking into account return to location of origin or to equivalent locations as affected by thermal motion. Because of the level of complication, this task will require a multi-year effort. Initially, the inter-atomic potentials for the materials of interest have been researched and the modeling requirements stemming from their temperature dependence will now be assessed. Later on, a new computer code will be written or an existing one will be modified to incorporate thermal motion into the modeling of collisions and subsequent atomic motions and settling in new lattice positions. Annealing will also be modeled toward the end of the task. So that this task can be kept to a manageable size, the methods to be developed will target only one or two materials. The principal material to be addressed will be graphite. In addition, silicon carbide ( $\mathrm{SiC}$ ) will be considered if time permits and data and suitable inter-atomic potentials can be obtained.

In addition to the effects discussed above, it is well recognized that the accumulation of damage in graphite manifests itself in increasing stored energy. If stable damage formations are created at the operating temperatures of the VHTR/NGNP, then unplanned/unexpected increases in temperature (transients) could result in the release of this energy and the potential creation of a positive feedback situation. To estimate the impact of this effect on reactor safety and operational characteristics, it is 


\begin{tabular}{|c|lll|}
\hline NEXT GENERATION NUCLEAR PLANT & Identifier: & PLN-2498 \\
METHODS RESEARCH AND & Revision: & 1 & \\
DEVELOPMENT TECHNICAL & Effective Date: & $9 / 25 / 08$ & Page: 72 of 188 \\
PROGRAM PLAN & & & \\
\hline
\end{tabular}

essential to determine its time behavior. Clearly, effects characterized by long-time constants (i.e., slow effects) will be easier to remedy than prompt effects characterized by extremely short-time constants.

The needed studies in this work can be performed using classical molecular dynamics (MD) simulations running on parallel computers. At the heart of the MD simulation is the choice of the potential function. The gradient of this function with respect to atomic displacement determines the forces on each atom. The integration of the equations of motion of the interacting particles yields the trajectory information. Consequently, the choice of the appropriate potential function is essential for the fidelity of the simulation. In general, many-body potentials are used to describe materials such as metals and semiconductors, and a few have been used for graphite. In addition, it is possible that potential functions can be constructed using $a b$ initio quantum mechanical simulations and subsequently used in the MD calculations. These options will be explored in this work.

\subsection{Improvement, Validation, and Verification (V\&V) of Code Suite}

The resulting suite of deterministic codes developed above will be verified against reference solutions obtained using Monte Carlo and deterministic models and against integral experiments. The reference (numerical) solutions will enable the accuracy of specific assumptions and approximations to be tested and verified. The double heterogeneity treatment will be examined for detailed fuel block and pebble problems by comparing the lattice code solutions with continuous-energy Monte Carlo solutions. The whole-core solution scheme will be verified against multi-group Monte Carlo solutions using precalculated multi-group cross sections and homogenized fuel-element models. The pebble-bed reactor solution will also be compared against results from the code V.S.O.P.

As the improvements and the extensions are implemented, the overall accuracy of the resulting suite of codes will be quantified by analyzing appropriate integral physics experiments. All known reactors, critical facilities, and other experiments of both types have been assessed for suitability as benchmarks. Similar assessments have been done for existing high-temperature reactors data by ANL and INL. For the prismatic reactor, the HTTR facility in Japan possesses large amounts of critical reactor physics data that can be used for validation purposes. For the pebble-bed reactor, the HTR-10 and PROTEUS may also provide essential data. HTR-10 has in fact been selected as the initial candidate for a full evaluation under the integral benchmark data task, as noted previously to be followed by PROTEUS funded under the NGNP project. In an independent effort, a set of neutronic and thermal-hydraulic benchmarks for the PBMR was initiated in $2005 \mathrm{OECD/NEA}$. This benchmark effort has been a cooperative effort among PBMR, (Pty), Ltd., Purdue University, Penn State University, and the NRG Corporation of the Netherlands and INL. Formal participation by INL began in June of 2005 when the PBMR benchmark workshop was held in Paris in conjunction with an OECD/NEA meeting. This benchmarking effort will conclude in 2008 when the participants will hold their last meeting in conjunction with the PHYSOR meeting in September 2008 in Interlaken (Switzerland).

Validation and verification of the tools used for these predictions can be accomplished through the collection of a large compendium of relevant in-core critical experiment data into a detailed, peerreviewed standard format as described previously in connection with the IRPhEP. USDOE-NNSA has also taken such an approach in handling the validation and verification for stockpile stewardship where computer modeling is also relied upon extensively. In support of this effort, it would be appropriate to ultimately establish and promulgate validation and verification standards, or at least some set of test problems, for the Generation IV systems. If suitable validation and verification data do not exist, experiments will have to be designed and conducted to fill in the gaps. 


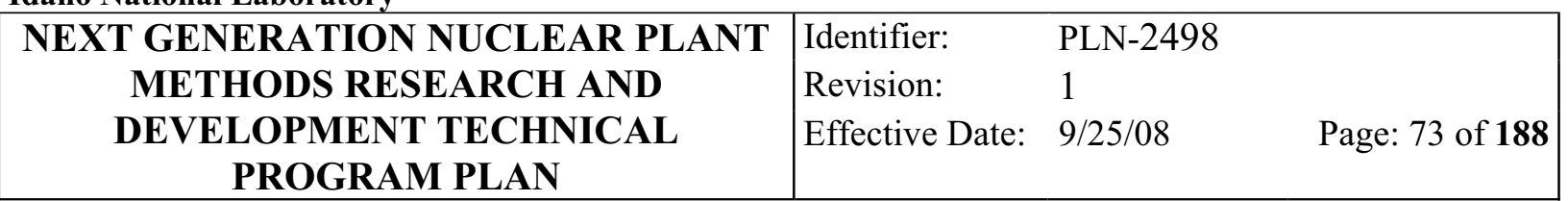

Monte Carlo simulation itself provides a powerful tool for validation and verification. The recent and continuing growth in computer power motivates the assessment and further development of Monte-Carlobased analysis capabilities applicable to multiple reactor types. Enhancement of these codes would also be investigated, including the propagation of errors as a function of depletion, provision of temperature interpolation capability, and modeling of thermal-hydraulic feedback.

The 3-D whole-core transport code DeCART was developed based on the method of characteristics for LWR applications at KAERI under an I-NERI project. This code eliminates the approximations and laborious multi-group constant generation stage of the two-step approach by representing local heterogeneity explicitly without homogenization using a multi-group cross-section library directly without group condensation, and incorporating pin-wise thermal-fluid feedback. With the extension of the geometry-handling capability and the inclusion of double-heterogeneity treatment, this code could be used as the reference tool for verifying and validating the nodal codes along with the partial use of the Monte Carlo solutions. The DeCART code could also be used as a lattice physics code for generating group constants. Thus it would have the dual functionality of group constant generation and whole-core calculation. An adaptation of the DeCART code for VHTR analyses was pursued under an I-NERI collaboration with KAERI. This will leverage the U.S. cost for this effort.

The required enhancements to the initial suite of codes, as identified by the validation and verification effort, will be implemented. This activity will be continuous and is tied to the validation and verification effort.

Experimental Facilities for Methods Validation: Recent evaluations have indicated that the operating range of the VHTR (temperature and burnup) will likely require an extensive experimental component to validate the reactor physics calculations, including the Monte Carlo ones. The cost of this effort will depend on the type of facility needed for such experiments, but would likely require significant funding. As a first approach, attempts should be made to leverage international activities (e.g., HTTR, HTR-10, ASTRA data, and planned activities by U.S. partners in the Gen IV International forum). If this is insufficient for code validation, an expert group consisting of analysts and experimentalists would be convened to provide justifications for the experiments and estimates of the associated costs. A more complete discussion of the efforts required is presented in Section 4.1.1.

\subsection{Summary: Focus and Direction}

Based on the rationales described above, this project will focus on the development of two suites of deterministic code systems consisting of spectrum codes: a lattice physics code and nodal diffusion codes that can be used for efficient and accurate design of the VHTR. Three-dimensional transport codes will be used when appropriate for generating assembly parameters or in benchmarking established designs. In order to accomplish the project goal efficiently, whenever appropriate, existing codes will be used as the basis of the new code suites with the addition of required functionalities for VHTR applications. ANL is developing a prismatic VHTR analysis capability based upon the DIF3D/REBUS-3 code, which has been successfully used for the reactor physics analyses of fast reactors, as well as LWRs. Similarly, INL is developing a pebble-bed analysis capability centered around the PEBBED code and prismatic capability embodying the same overall architecture as the PEBBED suite, but substituting a hexagonal-z nodal solver in the proper location. Although these codes provide the starting point of this project, a significant amount of development is required to enhance their capabilities and to equip a group-constant generation system that properly incorporates all of the physics of the two concepts. Many of the issues to be addressed in this effort are common to both concepts, yet there are distinguishing features that require parallel developments. The timelines for these activities are shown in Figure 21. 


\begin{tabular}{|c|lll|}
\hline NEXT GENERATION NUCLEAR PLANT & Identifier: & PLN-2498 & \\
METHODS RESEARCH AND & Revision: & 1 & \\
DEVELOPMENT TECHNICAL & Effective Date: & $9 / 25 / 08$ & Page: 74 of $\mathbf{1 8 8}$ \\
PROGRAM PLAN & & & \\
\hline
\end{tabular}

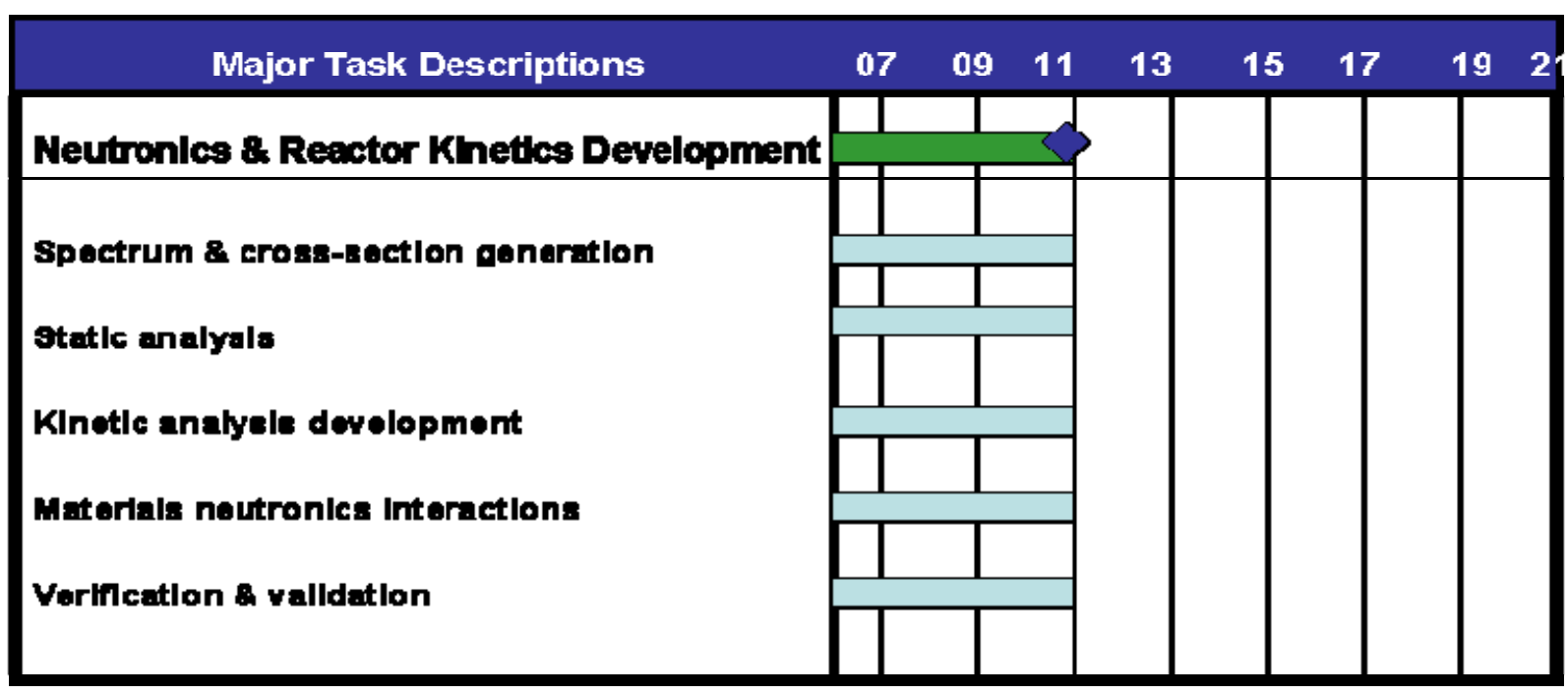

\section{Task completlon}

Figure 21. Time line for neutronic and reactor kinetics analysis development. 


\begin{tabular}{|c|lll|}
\hline NEXT GENERATION NUCLEAR PLANT & Identifier: & PLN-2498 & \\
METHODS RESEARCH AND & Revision: & 1 & \\
DEVELOPMENT TECHNICAL & Effective Date: & $9 / 25 / 08$ & Page: 75 of 188 \\
PROGRAM PLAN & & & \\
\hline
\end{tabular}

\section{THERMAL-FLUID BEHAVIOR AND STRUCTURE TEMPERATURES}

Flow and heat transfer in the VHTR are characterized by complex physics in complex geometries. Advanced simulation tools are available to simulate turbulent flow and heat transfer in engineered systems, and it is desired to validate such tools to determine their usefulness for applications to the VHTR. It is fully expected that advanced CFD codes will be needed to simulate regions of complex turbulent flow in the NGNP. Despite the size and complexity of the NGNP, it is currently expected that thermal-hydraulics systems analysis codes can be applied, in conjunction with CFD codes, to fully analyze the NGNP. The distinction between CFD and systems analysis codes stems from the distinctions between the software tools themselves. CFD codes use first-principle based solutions and subdivide a problem domain into cells that are small with respect to the phenomena that requires modeling. Systems analysis codes use field equations that have been simplified (for example by not including the viscous stress terms) and subdivide the problem into a macroscopic structure that does not model phenomena such as turbulent eddies. Neutronics/fluid behavior interaction will also be important to analyze in the VHTR.

The methodology applied to ensure that the thermal-hydraulic software can be used with confidence to calculate the behavior of the VHTR is outlined in Figure 22. However, it is useful to outline how the methodology will be applied specifically for the thermal-hydraulic R\&D outlined in this section-since the thermal-hydraulics R\&D, including the following summaries, stems directly from this methodology.

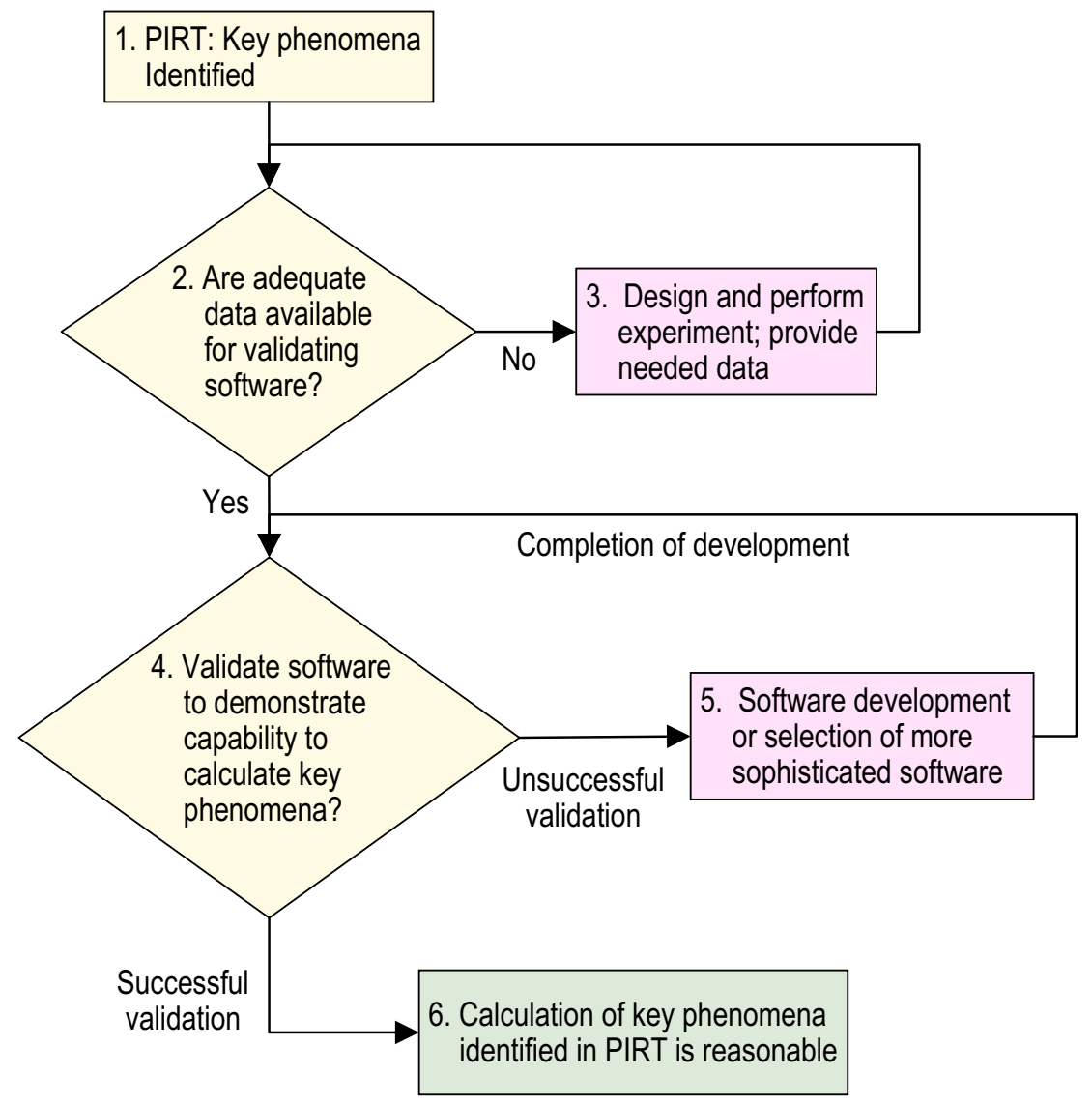

Figure 22. Thermal-hydraulic software validation methodology. 


\begin{tabular}{|c|lll|}
\hline NEXT GENERATION NUCLEAR PLANT & Identifier: & PLN-2498 \\
METHODS RESEARCH AND & Revision: & 1 & \\
DEVELOPMENT TECHNICAL & Effective Date: & $9 / 25 / 08$ & Page: 76 of 188 \\
PROGRAM PLAN & & & \\
\hline
\end{tabular}

The R\&D process is progressing as follows:

- $\quad R \& D$ Based on the Latest PIRT. Presently, the only available PIRT is the "first-cut" PIRT given in Table 6 (see Appendix A). However, as the design of the VHTR matures, an increasingly more sophisticated PIRT will be required to identify the key scenarios and important phenomena shown in Step 1 of Figure 22. Hence, the R\&D plan is based on the assumption that an ever-improving PIRT will be available. Clearly, all phenomena that must be calculated have not yet been identified. A formal PIRT should be created in conjunction with the preconceptual design in approximately 2007 or 2008 and then updated as the conceptual design, preliminary design, and final designs are formulated. Additional discussions on the upcoming PIRT requirements are summarized below.

- Software Validation. The software used to analyze the VHTR behavior must be validated for important scenarios. The process thus begins using existing data. If either existing data are not available or the existing data are not adequate to cover the VHTR's operational envelope, the experiments must be defined and built and data must be produced to provide the basis for software validation (see Figure 22, Steps 2 and 3). Hence, the first R\&D categories discussed below (Section 6.1) are experimental. Work to support the validation of the software tools used to calculate thermalfluids behavior are discussed in Section 6.2.

- Software Development. If validation studies show that the software cannot adequately calculate the key phenomena in the important plant scenarios, development must be done to improve the software or more sophisticated software must be used if available, or developed if not available (Figure 22, Steps 4 and 5).

- Analyses. Once the software has been validated and shown to be capable of calculating the important phenomena to the accuracy required (Figure 22, Step 6), then best-estimate analysis may begin.

Software validation, development, and analyses (Items c and d) for both the computational fluid dynamics and systems analysis codes are summarized in Sections 6.2.

Both the experimental research areas and the software-directed research areas are focused on the high-priority R\&D areas identified in the first-cut PIRT, as outlined in Table 6 , where key regions of concern are identified. In each case, the issues are whether the system will survive, particularly under the most challenging accident conditions, and whether the system will have an adequate operational lifetime for the conditions that are postulated (rated operational conditions, off-normal operational conditions, and accident conditions). The high-priority research areas include: (1) the core heat transfer, (2) mixing in the upper plenum, as well as the lower plenum, hot duct, and turbine inlet, (3) the heat transfer in the RCCS, (4d) air ingress following a system depressurization, and (5) the behavior of the integral system during the key scenarios, including the contributions of the balance-of-plant. These R\&D areas are outlined in Table 6 together with a summary of the key needs.

The $R \& D$ areas, including the relevant $R \& D$ tasks and the specific needs, are discussed in more detail in Sections 6.1.1 through 6.1.5. For each of the R\&D topics, study areas have been assigned (see 3rd column of Table 6) that indicate whether the R\&D is experimental (an activity designed to produce validation data); CFD code-related; or systems analysis code-related.

The first-cut PIRT focuses solely on the phenomena that are expected to dominate within the reactor itself and does not include any phenomena that may occur in the balance-of-plant or an intermediate heat exchanger (IHX) if one is present. While it is likely that the phenomena that present the most significant risk to the safety of the facility occur within the core, phenomena resulting from equipment failures in the balance of plant or flow instabilities in an IHX might also have significant impacts on the safety of the 
Idaho National Laboratory

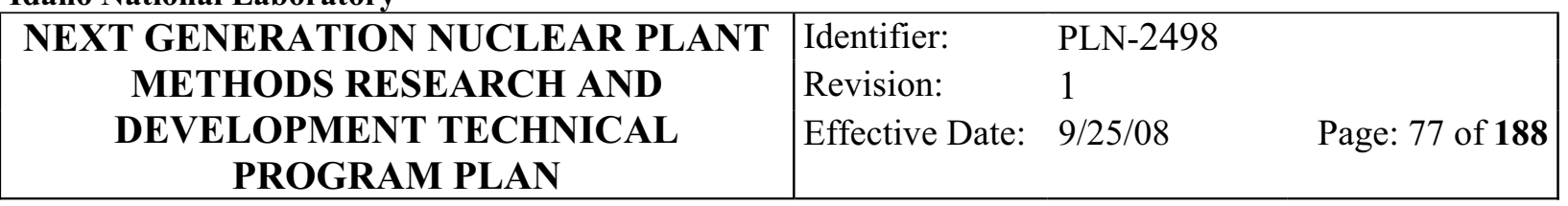

facility and may be included in a future PIRT. In particular, the nuclear-chemical coupling IHX, which provides the heat transport to the hydrogen production plant, similar to the core, has many coolant channels that could produce flow instabilities. The IHX will have inlet and outlet plenums, with mixing and stratification phenomena, so the reactor vessel plenum experiments could also apply in this case.

Table 6. Thermal-fluids methods R\&D areas.

\begin{tabular}{|c|c|c|c|}
\hline R\&D Area & Related R\&D & Study Area & Need \\
\hline $\begin{array}{l}\text { 1. Core Heat } \\
\text { Transfer }\end{array}$ & $\begin{array}{l}\text { Mixed convection } \\
\text { experiment, heated } \\
\text { experiments, core heat } \\
\text { transfer modeling, bypass } \\
\text { experiments, system } \\
\text { performance enhancements, } \\
\text { Sana experiments. }\end{array}$ & $\begin{array}{l}\text { Experimental }(\mathrm{E}), \\
\text { CFD, and systems } \\
\text { analysis codes }(\mathrm{S})\end{array}$ & $\begin{array}{l}\text { The core heat transfer, both with cooling flow } \\
\text { (operational conditions) and without cooling flow (DCC } \\
\text { and PCC), are instrumental in setting the maximum } \\
\text { temperature levels for fuel and material R\&D (core } \\
\text { graphite, structural materials, and heat load to RCCS). } \\
\text { The core heat transfer will determine the material } \\
\text { selection and configuration in the VHTR core, vessel, } \\
\text { and RCCS designs. }\end{array}$ \\
\hline $\begin{array}{l}\text { 2. Upper and } \\
\text { Lower plenums } \\
\text { (UP and LP) }\end{array}$ & $\begin{array}{l}\text { HTTR UP and LP, HTR_10 } \\
\text { UP and LP, MIR, heated } \\
\text { experiments, scaled vessel, } \\
\text { jets and cross-flow data, } \\
\text { upper plenum experiments, } \\
\text { system performance } \\
\text { enhancements. }\end{array}$ & $\mathrm{E}$ and CFD & $\begin{array}{l}\text { Circulation in the upper plenum is important during the } \\
\text { PCC scenario, since hot plumes rising from the hot core } \\
\text { may impinge on the upper head structures and lead to a } \\
\text { potential overheating of localized regions in the upper } \\
\text { vessel. } \\
\text { The degree of lower plenum mixing determines both the } \\
\text { temperature variations and maximum temperatures that } \\
\text { are experienced by the turbine blades, the lower plenum, } \\
\text { hot duct, and power generation vessel structural } \\
\text { components. Lower plenum mixing will determine the } \\
\text { material selection and configuration in the NGNP lower } \\
\text { plenum, hot duct, power generation vessel, and turbine } \\
\text { designs. }\end{array}$ \\
\hline 3. RCCS & $\begin{array}{l}\text { ANL (air-cooled), Seoul } \\
\text { National University (SNU; } \\
\text { water-cooled), HTTR RCCS, } \\
\text { fission product transport, } \\
\text { system performance } \\
\text { enhancements }\end{array}$ & $\mathrm{E}, \mathrm{CFD}$, and $\mathrm{S}$ & $\begin{array}{l}\text { The heat transfer efficiency of the RCCS will determine } \\
\text { the overall design concept (whether air-cooled is } \\
\text { sufficient or water-cooled is required in accordance with } \\
\text { either a confinement or containment RCCS design), plus } \\
\text { material selection of outer vessel wall, coatings } \\
\text { (selection of materials with emissivities that change with } \\
\text { surface temperature), natural circulation characteristics, } \\
\text { etc. }\end{array}$ \\
\hline 4. Air Ingress & $\begin{array}{l}\text { Diffusion model } \\
\text { development, NACOK } \\
\text { experiment }\end{array}$ & $\mathrm{E}, \mathrm{CFD}$, and $\mathrm{S}$ & $\begin{array}{l}\text { A gas-cooled VHTR should be able to survive the most } \\
\text { challenging accident scenarios with minimal damage and } \\
\text { thus should be able to resume operation in a minimum } \\
\text { time frame. The system must be shown to sustain } \\
\text { minimal damage following potential air ingress into the } \\
\text { core region. }\end{array}$ \\
\hline $\begin{array}{l}\text { 5. Integral } \\
\text { System } \\
\text { Behavior }\end{array}$ & $\begin{array}{l}\text { HTTR, HTR-10, AVR, } \\
\text { fission product transport, } \\
\text { CFD and systems analysis } \\
\text { code coupled calculations, } \\
\text { behavior of balance-of-plant } \\
\text { components (intermediate } \\
\text { heat exchanger, turbine, } \\
\text { compressor, reheater), } \\
\text { analyses of preconceptual, } \\
\text { conceptual, preliminary, and } \\
\text { final designs }\end{array}$ & $\mathrm{E}, \mathrm{CFD}$, and $\mathrm{S}$ & $\begin{array}{l}\text { The ultimate system characterization, to show the final } \\
\text { design is capable of meeting all operational expectations } \\
\text { and of surviving the most challenging accident } \\
\text { conditions, is performed using validated software tools. } \\
\text { The tools consist of the neutronics and thermal- } \\
\text { hydraulics software (coupled CFD and systems analysis } \\
\text { software) used in concert. This step is the culmination of } \\
\text { the comprehensive R\&D effort outlined herein. }\end{array}$ \\
\hline
\end{tabular}




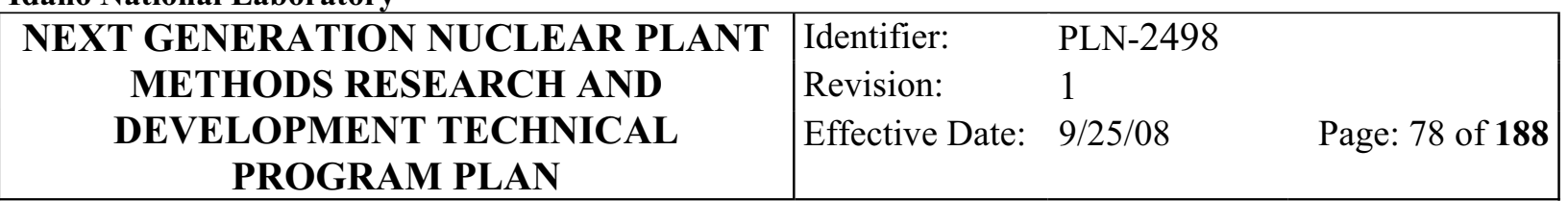

Flow instabilities may also be phenomena with complicated transient effects. At some point, as the PIRT progresses, there may be a future need to separate out a task for the development of transient CFD analyses techniques. As this could be a major undertaking, the budget may need to be adjusted accordingly at that time. What is acknowledged at this stage, even though the PIRTs have not reached this level of detail, is that local features in many cases determine the generation of the flow field turbulence structure. Design features to mitigate thermal stresses, promote mixing, enhance heat transfer, and reduce vibration are fluid-structure coupling mechanisms that need to be treated in CFD development.

In general, the $\mathrm{R} \& \mathrm{D}$ described herein is divided into two phases:

- Phase 1 focuses on codifying the methodologies, practices, and procedures for software validations. Phase $1 \mathrm{R} \& \mathrm{D}$ is designed to equally apply to either the pebble-bed or prismatic VHTR design.

Consequently, Phase $1 \mathrm{R} \& D$ includes experiments that are of a more fundamental nature and also analysis efforts designed to qualify software such as CFD, which has never been validated and used for nuclear system analysis purposes to any great extent in the past. Phase $1 \mathrm{R} \& \mathrm{D}$ will continue until a VHTR design is selected. Phase 2 will begin following design selection.

- Phase 2 focuses on executing the methodologies, practices, and procedures to validate the thermalfluids software and to perform the required analyses of the NGNP for final design studies, licensing, and plant operational studies.

Since the design will likely not be selected until late 2009 or early 2010, Phase 1 assumptions will govern the R\&D until at least this time frame.

\subsection{Introduction to CFD and Systems Analysis Code Validation Experiments}

The experiments that stem from the areas identified in Table 6 are described in Section 6.1.1-6.1.5. These experiments are aimed at producing validation data for CFD and systems analysis codes. Some potential issues identified to date include hot streaking in the lower plenum evolving from hot channels in the core (Figure 23), the geometric transition from the lower plenum into the outlet duct and the resulting temperature distribution in the short outlet duct, hot plumes in the upper plenum during pressurized cooldown (loss of flow accident), and parallel flow instability in the core during pressurized cooldown (Bankston 1965; Reshotko 1967). Several of these phenomena are pertinent to pebble-bed versions of the VHTR as well as the block versions. Although the geometry used as the basis for the first experiments is specific to the prismatic design shown in Figure 23, the strategy

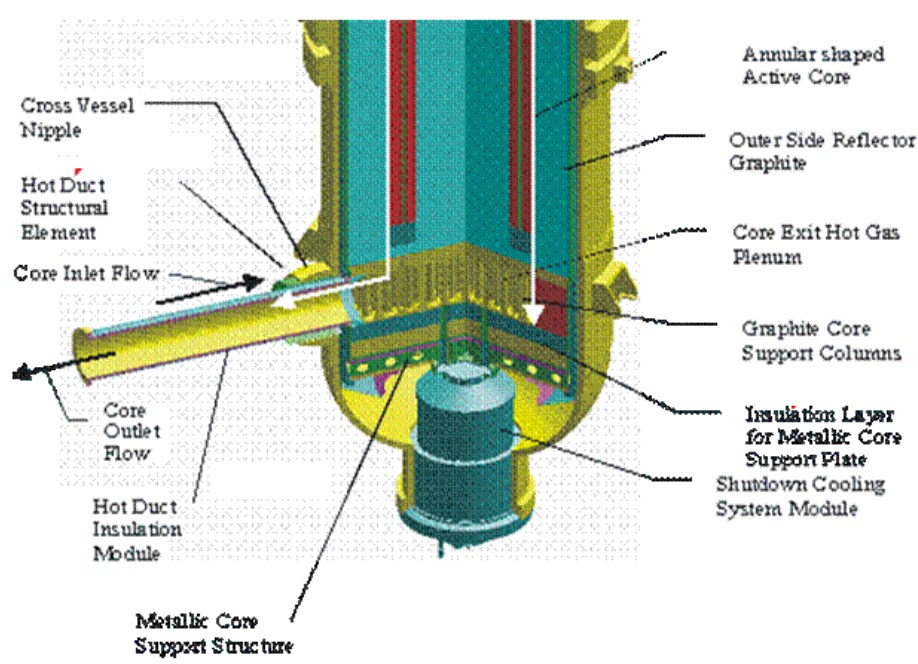

Figure 23. Core high power zones generate very hot exit gas and lead to high temperatures at turbine inlet or other power conversion equipment (IHX). for designing the code validation 


\begin{tabular}{|c|lll|}
\hline NEXT GENERATION NUCLEAR PLANT & Identifier: & PLN-2498 \\
METHODS RESEARCH AND & Revision: & 1 & \\
DEVELOPMENT TECHNICAL & Effective Date: & $9 / 25 / 08$ & Page: 79 of 188 \\
PROGRAM PLAN & & & \\
\hline
\end{tabular}

experiments is rooted in using scaling studies that will enable the resulting data to be directly related to other designs by using nondimensional parameters. This approach maximizes the relevance of the specified experiments to the design that is eventually selected, whether it is a pebble-bed or a prismatic design. The initial studies will concentrate on the coolant flow distribution through reactor core channels (hot channel issue) and mixing of hot jets in the reactor core lower plenum (hot streaking issue), phenomena that are important in both normal operation and accident scenarios. As VHTR development proceeds through various design, construction, licensing, and operation stages, the identification of new thermal hydraulic issues are anticipated.

Meaningful feasibility studies for VHTR designs will require accurate, reliable predictions of material temperatures to evaluate the material capabilities. In VHTR concepts, these temperatures depend on the thermal convection in the core and in other important components. Unfortunately, correlations in 1-D system codes for gas-cooled reactors typically under-predict these temperatures, particularly in reduced power operations and hypothesized accident scenarios. Conceptually, CFD codes with turbulence models can yield predictions for improving correlations and preliminary designs. However, most turbulence models in general-purpose CFD codes also provide optimistic predictions in that they under-predict resulting surface temperatures as shown in Figure 24 where the data are plotted as solid red squares (Mikielewicz et al. 2002; Richards, Spall and McEligot 2004]. These treatments are further complicated by the non-homogeneous power distributions with strong peaking that can occur and buoyancy, strong pressure gradients and gas property variations in the channels (hot channel issue).

Unfortunately, a universal turbulence model has not yet been developed so CFD predictions must be reassessed via experiments for each new complex situation encountered in the development of advanced reactors and their supporting systems. Further benchmark data are needed to avoid these problems and improve predictive capabilities in complex situations. These bases can be obtained from physical experiments or from numerical experiments such as direct numerical simulations (DNS) or large eddy simulations (LES), after validation with measurements. And, ultimately, prototypical integral experiments will be required for licensing confidence.

The general approach is to develop benchmark experiments needed for assessment in parallel with CFD and coupled CFD/systems code calculations for the same geometry. In each case, the benchmark experiments must be linked to the potential design by comprehensive scaling analyses that illustrate the relationships between the experiments and design in order to ensure the experiments yield benchmark data that are within the design's operational or postulated accident envelope.

The experiments defined below provide essential validation data in the following $R \& D$ areas:

- Core experiments:

- Turbulence and stability data from vertical cooling channels (2005-2009)

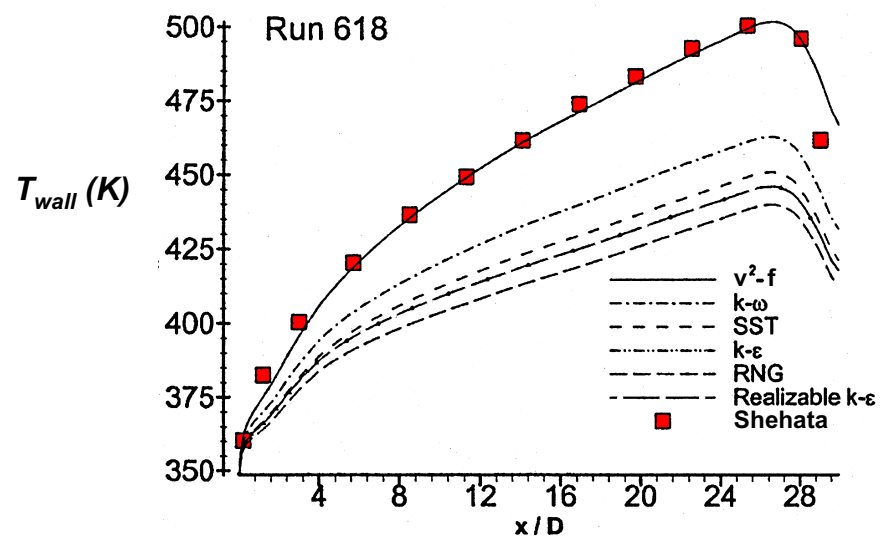

Figure 24. Preliminary assessment of popular turbulence models for flow in a vertical circular tube such as a prismatic NGNP cooling channel.

- Bypass flow studies (2008-2009) 


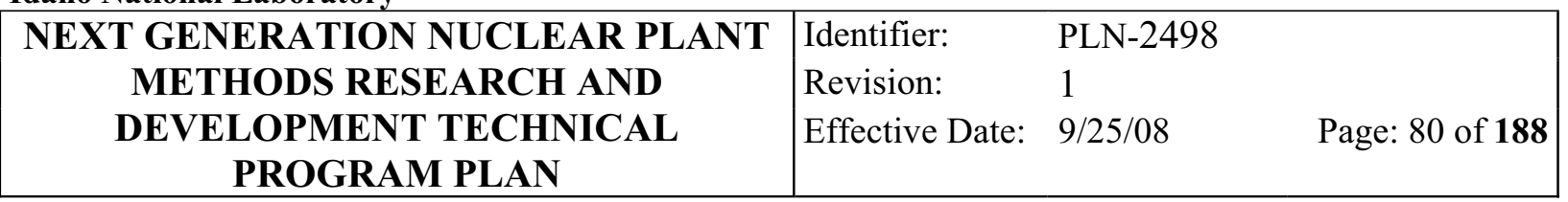

- Fluid dynamics of geometric transitions (2009-2011).

- $\quad$ Exit flows in pebble-beds (2007-2009)

- Pebble-bed experiments in NSTF

- Upper and lower plenum fluid behavior experiments:

- Lower plenum fluid dynamics experiments (2005-2009)

- Heated flows in lower plenums (2005-2009)

- Interactions between hot plumes in an upper plenum and parallel flow instabilities (2007-2010).

- Reactor cavity cooling experiments.

- Air ingress experiments:

(1) Stratified flow into the lower plenum (2008-2010)

(2) Heat transfer and pressure drop of mixtures of air and helium (2006-2008).

- Larger-scale vessel experiments:

- Integral experiments: HTTR and HTR-10

- New large-scale experiments:

(1) to examine the behavior in the core, in the plenums, and the interactions between them

(2) Prototypical heat transfer and fluid dynamics experiments to assist in concept selection (2008-2011)

(3) Large-scale experiments based on final design (2012-2017).

In general, two types of experimentation are planned: isothermal fluid dynamics measurements and heated flow studies. The purpose of the isothermal fluid dynamics experiments is to develop benchmark databases for validating CFD solutions of momentum equations, scalar mixing, and turbulence models for typical VHTR geometries in the limiting case of negligible buoyancy and constant fluid properties - when the flow is turbulent and momentum-dominated. ${ }^{1}$ The intent of the heated flow experiments is to provide data on the modifications of the thermal hydraulic behavior (and proposed turbulence models) as additional effects, such as gas property variation and buoyancy, become important. ${ }^{\mathrm{m}}$

The subsequent discussion is divided into five general areas: (1) core heat transfer including experiments 1a through 1e above, (2) upper and lower plenum fluid behavior including experiments 2a through $2 \mathrm{c}$ above, (3) reactor cavity cooling experiments, (4) air ingress experiments, and (5) large-scale vessel experiments including experiments $5 \mathrm{a}$ and $5 \mathrm{~b}$ above.

1. The matched-index-of-refraction experiment is designed to examine the flow behavior for phenomena where the momentum terms dominate and buoyancy is negligible.

m. Phenomena that are governed by density-gradients cannot be studied in the matched-index-of-refraction experiments. Instead, either heating must be used to define representative temperature gradients and thus representative fluid density gradients or different fluids must be used that will simulate the desired density gradients. 


\begin{tabular}{|c|lll|}
\hline NEXT GENERATION NUCLEAR PLANT & Identifier: & PLN-2498 \\
METHODS RESEARCH AND & Revision: & 1 & \\
DEVELOPMENT TECHNICAL & Effective Date: & $9 / 25 / 08$ & Page: 81 of 188 \\
PROGRAM PLAN & & & \\
\hline
\end{tabular}

Based on the limited applicability of the legacy thermal-fluids data base, in particular with respect to CFD, it is anticipated that approximately 20 to 50 new experiments will be required to validate the software needed to qualify the NGNP at its peak operational temperatures and efficiencies. The number of experiments is dependent on the availability of data sets applicable to the chosen design that are available from the reactor vendor and the software calculational uncertainty requirements. The number of required experiments increases with decreasing calculational uncertainty. Consequently, the experiments described in the following sections are new experiments that have not been performed to date and are not presently available elsewhere with only a few exceptions. Even for facilities that are available elsewhere in the world, such as the HTTR in Japan, HTR-10 in China, and RCCS facility in Korea, specially designed experiments will most likely be required for a complete software validation.

\subsubsection{Core Experiments}

\subsubsection{Vertical Cooling Channel Turbulence and Stability Experiments}

Experiments of this type will provide documented temperature, velocity, and turbulence field data for forced and mixed convection (buoyancy effects) and gas property variation in VHTR block-type reactor cooling channels in order to validate the turbulence models at reactor conditions for which benchmark data are not available. The proper calculation of turbulence directly influences cooling flow and temperature. Instrumentation will include miniaturized, multisensory, hot-wire probes developed as a task in a recent NERI project for gas-cooled reactors (McEligot et al. 2002). Both down-flow (normal operation) and up-flow (pressurized cooldown) will be considered.

Turbulence modelers request measurements of the basic quantities (dependent variables) of their governing partial differential equations for validation (and guidance), quantities such as turbulence kinetic energy and Reynolds stresses, etc. These data generally have not been available for internal flows with gas property variation. Experimental databases are currently available to assess some aspects of the hot channel problem, particularly forced convection, but not with details of the turbulence structure (McEligot 1986). Additional measurements will be obtained in ongoing university projects. These sources and the existing literature will be compared to the requirements identified above for qualified databases to determine which additional measurements are needed for heated gas flow in circular tubes. These data will be sought and, if of benchmark quality and available, will be acquired to reduce our experimental needs and costs. Unfortunately, many of the extensive archival publications on mixed convection (buoyancy influences) are not adequate to serve as databases for CFD assessment, and measurements needed to evaluate details for turbulence models in complex situations are lacking, as is the case with VHTR concepts. Ultimately sensitivity studies will be required to quantify the relative importance of factors that influence turbulence, including potential geometrical and hardware configuration variations.

For normal operation, the flow in the VHTR coolant channels can be considered to be dominant, turbulent-forced convection with slight transverse property variation. In a pressurized cooldown simulation, the flow quickly becomes laminar with some possible buoyancy influences (McEligot and McCreery 2004) and parallel flow instabilities may become important (Bankston 1965; Reshotko 1967). Flow is predicted to be upwards in the inner and middle rings and to remain downward in the outer ring; heat transfer may be to or from the gas, depending on location and timing. During the initial transient, the turbulent criteria are all predicted to remain below their thresholds for significant effects as in normal steady operations (McEligot and Jackson 2004). In contrast to turbulent flow, an "aiding" laminar flow (heated up-flow or cooled down-flow) enhances heat transfer parameters; an "opposing" flow reduces these parameters until it becomes unstable and undergoes transition to a turbulent-like flow. For opposing flow, Scheele and Hanratty suggest that above a threshold there is a transition to an asymmetrical flow 


\begin{tabular}{|c|c|c|c|}
\hline $\begin{array}{l}\text { NEXT GENERATION NUCLEAR PLANT } \\
\text { METHODS RESEARCH AND } \\
\text { DEVELOPMENT TECHNICAL } \\
\text { PROGRAM PLAN }\end{array}$ & $\begin{array}{l}\text { Identifier: } \\
\text { Revision: } \\
\text { Effective Date: }\end{array}$ & $\begin{array}{l}\text { PLN-2498 } \\
1 \\
9 / 25 / 08\end{array}$ & Page: 82 of $\mathbf{1 8 8}$ \\
\hline
\end{tabular}

with local separation at the wall and then transition to an unsteady and later intermittently turbulent flow; this situation cannot be predicted adequately with a systems code or a steady, axi-symmetric CFD code.

This experiment (Figure 25) will support the efforts of the current computational task concerning the hot channel issue by providing benchmark data for detailed assessment of its turbulence models for forced and mixed convection with helium property variation. The miniature multiple-sensor hot-wire probes from Profs. Wallace and Vukoslavcevic (2003; Figure 26) will be inserted through the open exit to obtain point-wise temperature and velocity measurements. Hence, our objectives are to measure the fundamental turbulence structure and to obtain benchmark data to assess CFD codes for high temperature gas flows that are in the forced and mixed convection regions, for a range of conditions important in VHTRs. To achieve these objectives the experiment will provide an approximately uniform wall heat flux boundary condition in a tube for helium, either ascending or descending and entering with a fully-developed turbulent velocity profile at a uniform temperature, as in coolant channels after passing through an end reflector.

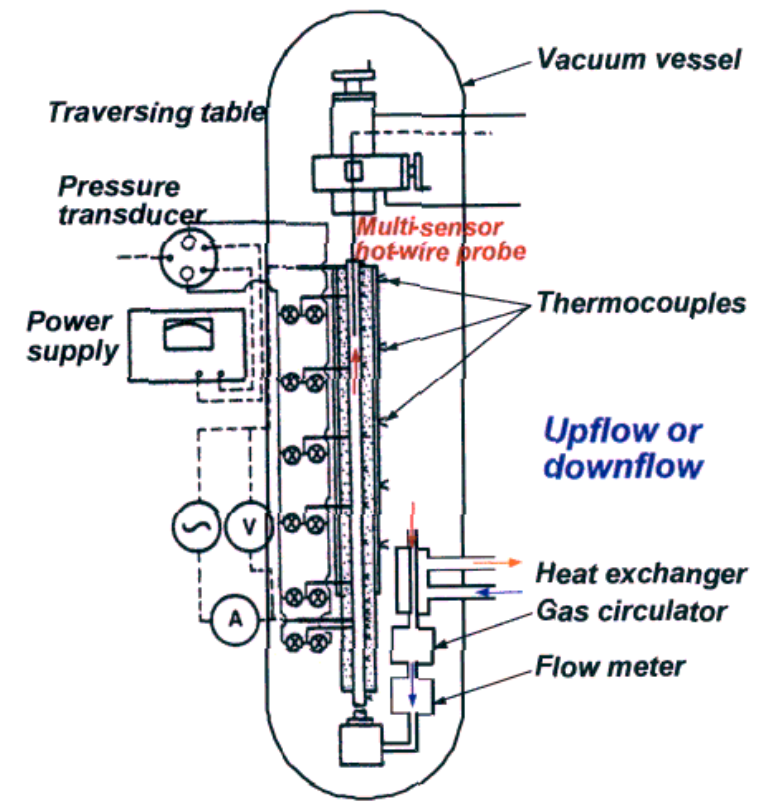

Figure 25. Potential apparatus to obtain benchmark turbulence data in heated channel flow.

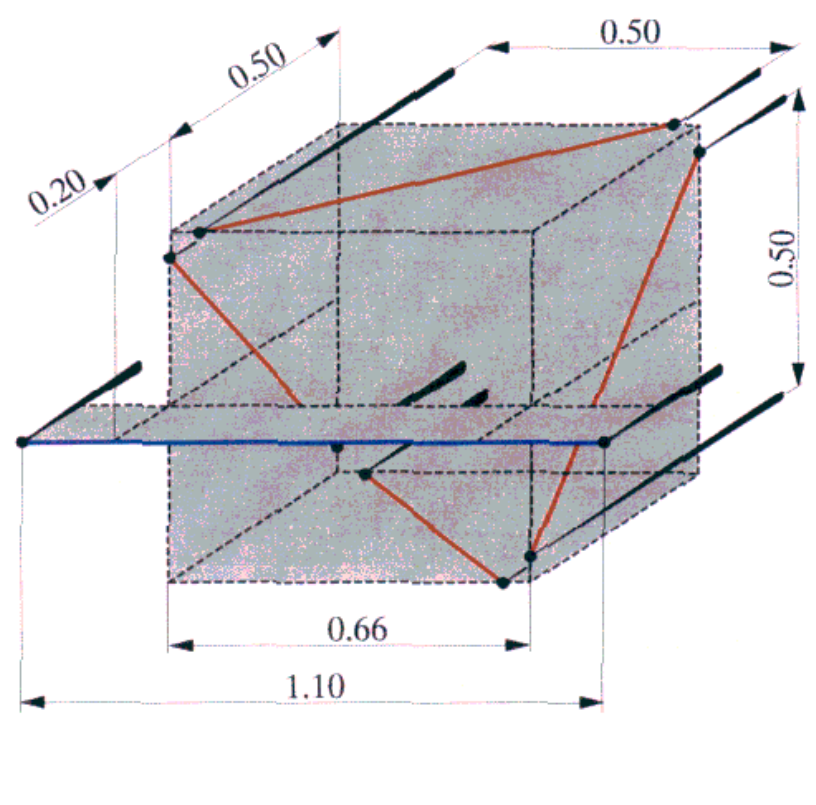

Figure 26. Schematic diagram of miniature fivesensor probe by Vukoslavcevic and Wallace (2003), the dimensions are in millimeters.

In addition to the above, it may be possible to perform depressurized conduction cool-down experiments in the NSTF for the prismatic block reactor to simulate the conduction and radiation of the decay heat away from the fuel in the core and transfer it to the primary vessel metal. In FY 2007, the existing NSTF facility will be evaluated at a high-level to explore the possibility of using this facility in a multifunctional capacity, to include these depressurized conduction cool-down tests for the prismatic block core.

The FY 2005 and 2006 studies included initiating preliminary design of the apparatus and estimating costs and schedules. Final design, reevaluation of costs and schedules and initiation of fabrication are planned for later years. Equipment operation, measurements, and documentation will occur in FY 2007 to 2009 timeframe. 


\begin{tabular}{|c|lll|}
\hline NEXT GENERATION NUCLEAR PLANT & Identifier: & PLN-2498 \\
METHODS RESEARCH AND & Revision: & 1 & \\
DEVELOPMENT TECHNICAL & Effective Date: & $9 / 25 / 08$ & Page: 83 of 188 \\
PROGRAM PLAN & & & \\
\hline
\end{tabular}

\subsubsection{Bypass Flow Experiments}

A series of experiments is envisioned that will test the various theories regarding factors that influence the quantity of bypass (in either the prismatic or PBR) as a function of various factors such as manufacturing tolerances and core configuration changes from irradiation or thermal expansion. The work scope is seen as a laboratory-university partnership with the large-scaled experiments being performed in the ANL NSTF.

The bypass is the core flow that moves through the core via the interstitial and noncooling passages in a prismatic reactor, through unanticipated zones of low resistance in a PBR, and through the reflector regions in both designs. Bypass may vary from 10 to $25 \%$ or more of the total core flow and will vary during the lifetime of the reactor as a function of the local temperature and changes in the dimensions of the prismatic reactor's graphite blocks because of irradiation damage or changes in the pebble-bed axial bed fraction because of lifetime variations in the loading patterns. Because the bypass flow exerts considerable influence on core temperatures and peak exit cooling channel jet temperature and thus the temperature distribution in the lower plenum at operational conditions, it is crucial to identify the NGNP core bypass characteristics and its influence on the reactor's peak temperatures.

The resolution for this phenomenological issue may well have to be a statistical approach similar to that used for the classical hot spot/hot channel factors. A high-level stochastic structure involving a combination of materials modeling, both first-principles and correlations, thermal-hydraulics R\&D, and manufacturing practice will need to be put in place early to guide the research of university participants. It is anticipated that university researchers will investigate the various factors that influence the bypass and develop preliminary models. NSTF bypass flow experiments are envisioned in FY 2009 and FY 2010 to test and confirm the various theories regarding factors that influence the quantity of flow bypassing the coolant passages and core (in either prismatic or PBRs). Functional dependences on factors such as manufacturing tolerances and core configuration changes caused by irradiation and thermal expansion will be determined. In such experiments, hardware may be built to represent core hardware that might result from prolonged irradiation and consequent nonuniform distortion.

For the case of the prismatic reactor, small-scale experiments encompassing both thermal-hydraulics and materials phenomena will be performed (perhaps at universities). Results from such experiments will be used in the selection of geometrical configurations and materials for the blocks and the flow condition for the large-scale NSTF bypass flow experiments. Up to $\sim 20 \mathrm{ft}$ of axial heated height is available to represent axial variations in gap width because of varying conditions. A number of columns of core blocks and reflector regions will be installed in the cavity test section of the NSTF to simulate parts of the core and the associated boundary elements. The size of the blocks, and the material selected-graphite or surrogate - will be determined by the scaling analyses.

For the PBR, small-scale experiments for detailed gas dynamic phenomena and measurement techniques will be performed (perhaps at universities). Results from these experiments will then be used in the selection of geometrical configurations and materials for both pebble and flow conditions for the large-scale NSTF bypass flow experiments. A number of pebbles (ping-pong balls) will be poured into the cavity test section after the installation of structure to simulate parts of the core and the associated boundary elements. The size and number of the pebbles, and the material selected will be determined by the scaling analysis. Up to $\sim 20 \mathrm{ft}$ of axial heated height is available to represent a variety of axial variations on coolant void fraction. The NSTF blower will provide forced air flow at atmospheric pressure. The scaling similarity to helium at 70 bars will be performed. Various temperature profile tests will be performed using the axially varying heater control and heater power. Instrumentation will be provided to measure the coolant flow distribution for both cases using a combination of LDV and 


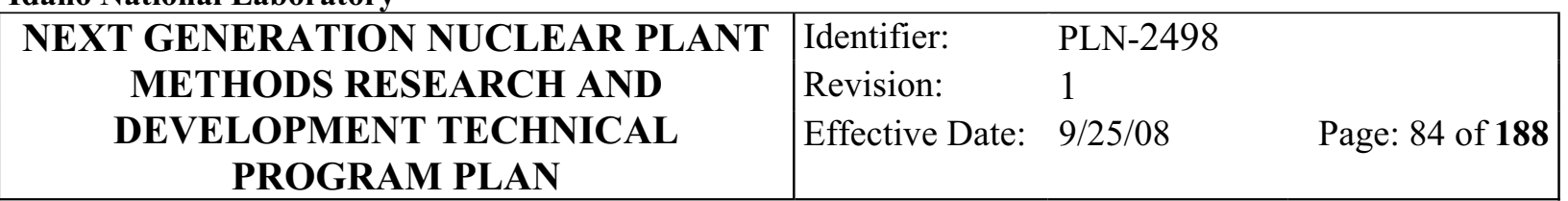

anemometry. The NSTF flow bypass experiments will be conducted in FY 2010 and 2011 with experiment fabrication in FY 2009.

\subsubsection{Fluid Dynamics of Geometric Transitions}

In typical prismatic VHTR concepts, the complicated transition from coolant channels to the lower plenum provides the inlet conditions for the jets into the lower plenum; measurements of turbulence distributions and pressure drop (loss coefficients) are needed for CFD predictions and design. Depending on the reactor designs, comparable problems may appear for the upper plenum. Also, at the geometric transition from a lower plenum to its outlet duct, the convergence may cause laminarization of the turbulent flow, leading to reduced thermal mixing (McEligot and McCreery 2004). Experiments on fluid dynamics of geometric transitions during FY 2009 through 2011will employ the INL MIR flow system (discussed separately under lower plenum fluid dynamics experiments) and gas flow experiments to address the key geometry problems identified in VHTR conceptual designs to that time, both for prismatic and pebble-bed approaches.

In addition, a series of scaled bench-top experiments may prove essential for the validation of multidimensional and/or multiphysics predictive computational simulation tools for the evaluation of design specific local features which improve the performance of the system, such as insulation, baffles, mixing plates some of which are specific to the core region. As candidate designs are developed, it is anticipated that a list of important local features that are likely to have a limiting effect on system performance will be developed for each design. At this stage, two such features that require simulation tool development and/or validation can be identified as important to all potential designs: (1) the prediction of isothermal flow-induced vibration of plate or sheet components (e.g., insulation or thermal radiation shielding) and (2) the prediction of flow-induced vibration and thermal distortion of plate or sheet components (e.g., insulation or thermal radiation shielding). In both cases, the wear and fatigue associated with these fluid-structure interactions may have significant implications on the expected life and the probabilistic failure rates of these components, which impact the safety case for the system as a whole. Additional important local features may be identified as potential designs mature. Local structural effects because of large depressurization accidents could be such a possibility.

\subsubsection{Exit and Wall Flows in Pebble-Beds}

An MIR experiment will be conducted to examine flows in pebble-beds near their outlets. A key difficulty in analyzing the safety of PBR systems is predicting the maximum fuel temperatures and chemical reaction rates locally in the coolant outlet region (e.g., hot spots) where the temperature field is generally high. Typically, 1-D system codes are applied for transient safety analyses and parameter studies during preliminary design (Oh, Moore, and Ambrosek 2000). A 1-D calculation predicts quantities that are averaged across the flow (e.g., the core diameter) and does not predict the highest temperatures or their locations. Further, since chemical reaction rates vary nonlinearly with temperature, the average reaction rate is not the reaction rate at an average temperature. While these systems codes are needed, it is desirable to supplement them with 3-D calculations for final designs and for estimating hotspot factors to improve their predictions. Potentially, 3-D CFD codes can be applied, using a porous medium approximation to find the coolant velocity and temperature in localized "macroscopic" regions. Then DNSs can be used to identify the point-wise peak temperatures and their locations (microscopic treatment). The goal of this research is to develop accurate techniques for predicting maximum temperatures in VHTR concepts that use pebble-bed technologies by coupling CFD calculations with experiments in the unique INL MIR flow system. 


\begin{tabular}{|c|lll|}
\hline NEXT GENERATION NUCLEAR PLANT & Identifier: & PLN-2498 \\
METHODS RESEARCH AND & Revision: & 1 & \\
DEVELOPMENT TECHNICAL & Effective Date: & $9 / 25 / 08$ & Page: 85 of 188 \\
PROGRAM PLAN & & & \\
\hline
\end{tabular}

The flow through a pebble-bed core is not unidirectional as in experiments to derive flow correlations. The general flow converges and diverges (in addition to the localized changes in direction at the pore-scale). However, it is well known to fluid physicists that a convergence stabilizes flows (Schlichting 1979); in a turbulent flow, the turbulence levels can be reduced below expectations and the flow can even be laminarized (McEligot and Eckelmann 1993; Satake et al. 2000). A consequence is a reduction in convective heat transfer coefficient and, hence, an increase in surface temperatures. While criteria for this occurrence have been hypothesized for turbulent boundary layer flows (Murphy, Chambers, and McEligot 1983), none is known to us for converging flows in porous media. Appropriate measurements are needed to quantify this phenomenon and, hence, to determine its importance in PBR technology.

Under accident conditions (no forced flow) heat is transported by radiation, heat conduction of the pebbles (through the contact points), and convection. An integral simulation can only be done using the porous medium approach. For this treatment several parameters are needed, such as pressure loss and volumetric heat transfer coefficient between gas and pebbles. A model that accounts for the turbulent mixing because of the complex path of the gas through the pebble array will be important. Such models have so far been developed on the basis of intuition; thus experiments are needed. An additional difficulty for predictive techniques near a converging outlet region is that, as the radius of the bed decreases, the effects of the surrounding wall increase relatively (Cheng and Hsu 1986). In the interior, an isotropic approach seems to be appropriate, but near the boundaries of the pebble-bed, the porosity becomes strongly nonisotropic. Here, the average porosity increases and the flow resistance decreases, resulting in channeling along the boundaries. Little is known about the current macroscopic models that take account of this effect (e.g., what is their accuracy?). A similar problem becomes important in the context of flow with heat transfer, because the boundary between a pebble-bed and plain wall may act as an insulation layer. The combined effect of the convergence and wall effect is another unknown that needs study. Measurements are needed to examine the validity of any models employed and their related constitutive theories. The INL MIR flow system is ideal to investigate these difficulties in detail.

The work planned for the period FY 2007 through FY 2009 will develop experiments to provide an understanding of converging porous flows, to assess codes predicting the flow in PBRs, and to physically model the outflow from pebble-beds. Experiments using refractive-index-matching have already been employed to examine flow and particulate transport in saturated, homogeneous porous media (Johnston, Dybbs, and Edwards 1975; Cenedese, Cushman, and Moroni 2002). Usually, the experiments have been small. Of interest for the current application are the results of Dybbs and Edwards (1984) who used flow visualization and LDV for flow through hexagonal packed beds of spheres with refractive-index-matching and defined four successive, distinct flow regimes. Despite the wide range of previous work on flow and heat transfer in porous media and in PBRs, a number of important scientific and technological needs remain in order to design and operate VHTR reactors using pebble-bed technology confidently and safely:

- Quantify the effect of side walls on the reduction of coolant flow resistance because of increased porosity and the regular arrangement of the spheres (for flow channeling)

- Evaluate the effects of coolant flow convergence (and divergence) on the stability of coolant flow in pebble-beds

- Evaluate effects of converging walls of pebble-beds on flow, heat transfer, and chemical reactions

- Physically model typical outflow regions from pebble-beds and obtain benchmark measurements.

The objectives of the INL laboratory study will be to answer the experimental aspects of the needs identified above for treating the coolant flow and transport through characteristic VHTR pebble-bed 


\begin{tabular}{|c|lll|}
\hline NEXT GENERATION NUCLEAR PLANT & Identifier: & PLN-2498 & \\
METHODS RESEARCH AND & Revision: & 1 & \\
DEVELOPMENT TECHNICAL & Effective Date: & $9 / 25 / 08$ & Page: 86 of 188 \\
PROGRAM PLAN & & & \\
\hline
\end{tabular}

geometries, including investigation of a larger range of Peclet and Reynolds numbers than has been previously obtained but are badly needed. The experimental model and apparatus will be designed and fabricated using a variety of new and existing equipment. The MIR test section would be filled with pebble-beds of differing permeabilities consistent with scaling considerations. The current combination of quartz and light mineral oil will be employed for refractive index matching.

Flow through the model will be provided by an existing auxiliary flow system with its own temperature control system for adjusting the refractive index of the oil passing through the porous media. For optical access, oil flow is maintained in the main loop and its temperature control system is employed to ensure that the refractive indices are matched from the test section walls to the model wall. Measurements will be obtained by particle image velocimetry (PIV), laser Doppler velocimetry (LDV), by video and/or camera recording and by Particle Tracking Velocimetry (PTV) with a Moving Particle Tracking system (MPT). Instantaneous velocity components will be measured by LDV at fixed positions. Three experiments will be constructed, two using the MIR flow system and one with airflow for initial evaluations:

- MIR measurements of converging flow in pebble-beds

- Pressure distribution data in air flow corresponding to Experiment (1)

- Physical modeling of typical outlet region by MIR benchmark measurements.

Typical results will include time-resolved, point-wise distributions of the mean velocities, $\mathrm{U}, \mathrm{V}, \mathrm{W}$, and their Reynolds stress components for assessment of proposed CFD codes. The applicability of using oil (or any Newtonian fluid to represent the chosen reactor working fluid) instead of helium, is confirmed by using equivalent ranges of nondimensional parameters in nondimensional forms of the field equations. Because all of the fluid behavior occurs at low Mach numbers, the flow is treated as incompressible.

\subsubsection{Pebble-Bed Experiments in NSTF}

It may be possible to utilize the NSTF to perform complementary experiments to better characterize the heat transfer in the pebble bed. Preliminary discussions indicate that perhaps electrical heating may be employed to study the various heat transfer mechanisms and their distribution to supplement the data recorded in the PBMR Pty experiments. If such experiments are shown to be feasible, construction could begin in FY 2009 and benchmark data to assist the validation process should become available in FY 2010 and FY 2011.

\subsubsection{Upper and Lower Plenum Fluid Behavior Experiments}

A number of experiments focused on analyzing typical behavior in the lower and upper plenums are summarized in the following subsections. Although the specific lower and upper plenum geometries have not yet been specified, it is known that the reactor will most probably have both a lower and upper plenum. Also, the final lower and upper plenum geometry designs, whether the reactor is a pebble-bed or a block-type configuration, will probably have features similar to the baseline design that is being used to define the preliminary experiments. That is, the upper plenum will probably have accommodations for inserting control rods and also a number of flow channels will be available for the working fluid to proceed through the core. Although the aspect ratio (height to diameter) will probably be different than that chosen, it is probably safe to assume that the flow making the transition in the plenum to the core will not be developed flow. For the lower plenum, there will probably be various flow obstructions in place whose function is to provide structural support for the core hardware and the flow will likely exit through a duct such that the core flow will be required to shift direction from downward to a horizontal direction. 


\begin{tabular}{|c|lll|}
\hline NEXT GENERATION NUCLEAR PLANT & Identifier: & PLN-2498 \\
METHODS RESEARCH AND & Revision: & 1 & \\
DEVELOPMENT TECHNICAL & Effective Date: & $9 / 25 / 08$ & Page: 87 of 188 \\
PROGRAM PLAN & & & \\
\hline
\end{tabular}

Finally, the flow characteristics will likely be quite different from one side of the lower plenum to the other side because of the siting of an exit duct on the side of the reactor vessel. Thus, the validation data produced in the experiments described below are, to a degree, scalable to the final design geometry.

\subsubsection{Lower Plenum Fluid Dynamics Experiments}

Accurate predictions of the thermal mixing in the lower plenum are needed to predict the temperature distribution of the core outlet duct and its material behavior. Because of the variation of power and the heat generation across and along the core, the jets from the cooling channels into the plenum may vary substantially in temperature. If the turbulent mixing of these flows is incomplete, high temperature gas may impinge on lower plenum surfaces and/or the entrance of the outlet duct causing potential structural problems. Nonuniformity of the temperature distribution in the gas may also adversely affect the intermediate heat exchanger or high-pressure turbine. The geometric transition from the circular cooling channels in the core to the lower plenum is complex, as is the configuration of the lower plenum itself with its array of posts supporting the core. Plenum studies are pertinent to PBRs as well as prismatic block versions, even though the details of the lower plenum designs can be expected to differ. Hence, reliable, accurate data are needed to validate predictive techniques for the flow and mixing in the plenum.

To partially address the above concerns, an experiment to study fluid dynamics and mixing in a lower plenum has been designed and should be used to generate data by the end of FY 2005. The experiment design is being undertaken by considering the flow in the lower plenum to consist of multiple jets into a confined, density-stratified, cross-flow with obstructions. Since the flow converges ultimately to a single outlet, the hot jets encounter different cross-flow velocities, depending on their locations relative to the outlet. Possible flow routes in the lower plenum of a typical block reactor design concept are shown in plan view in Figure 27.

As described in the previous section, the choice of experimental working fluids may be postponed, since whatever the fluid chosen its behavior is related to the VHTR reactor working fluid using similar operational ranges as determined using nondimensional parameters in the applicable nondimensional field equations.

The large circles represent support posts while the smaller ones identify locations of the inlet ducts from the cooling channels in the active core. Some bypass flow can also be expected to enter the lower plenum after passing vertically between the hexagonal graphite blocks both in the core and the reflectors. The arrows give intuitive examples of some paths the flow could be expected to take through the lower plenum from the far side to the outlet duct. In some regions the flow pattern would be comparable to cross-flow over a triangular array of rods as in a shell-and-tube heat exchanger; in other locations the flow may tend along passageways formed by parallel rows of posts. The flow rate (or Reynolds number) increases from the right side of the figure to the left as more incoming jets participate.

The jets furthest from the outlet essentially exhaust into stagnant surroundings between the adjacent posts with the exception of the flow

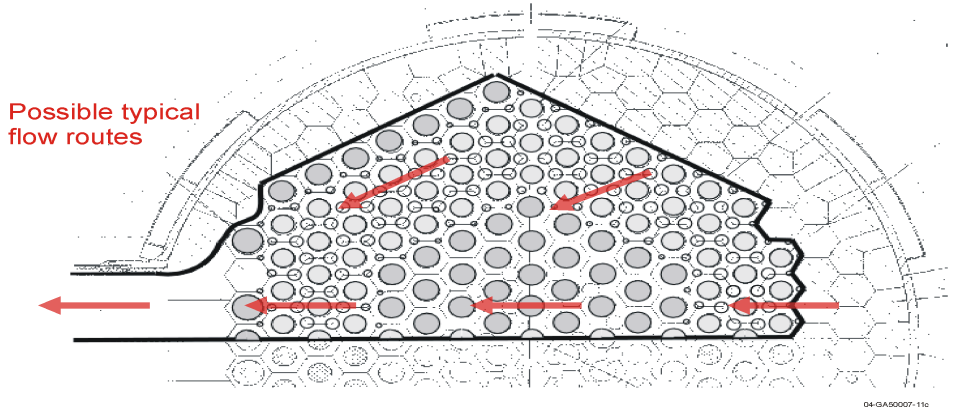

Figure 27. Examples of some possible flow paths in the lower plenum of a typical VHTR block reactor concept. that they induce. If a hot channel region is exhausted via one of the furthest jets, there is concern that its 


\begin{tabular}{|c|lll|}
\hline NEXT GENERATION NUCLEAR PLANT & Identifier: & PLN-2498 & \\
METHODS RESEARCH AND & Revision: & 1 & \\
DEVELOPMENT TECHNICAL & Effective Date: & $9 / 25 / 08$ & Page: 88 of 188 \\
PROGRAM PLAN & & & \\
\hline
\end{tabular}

impingement on the floor of the lower plenum may be too hot for the insulation layer protecting the metallic core support plate below. On the other hand, the last row of jets before the outlet encounters cross-flow from all the other jets. The hot streaking issue pertains primarily to the entrance of the hot outlet duct to the turbomachinery. If a hot channel region exhausts through one of the last jets before the outlet duct, there is concern that it may not mix (and thereby cool) sufficiently before flowing along the metallic outlet duct.

Data from a number of separate effects experiments appear to be available for initial assessment of the capabilities of CFD codes to handle some individual phenomena in a lower plenum (Schultz, Ball, and King 2004). The proposed INL MIR studies are aimed at taking the next step, that of providing databases for key coupled phenomena such as jet interactions with nearby circular posts and with vertical posts in the vicinity of vertical walls with near stagnant surroundings at one extreme and significant cross-flow at the other.

Current prismatic VHTR concepts have been examined to identify proposed flow conditions and geometries over the range from normal operation to decay heat removal in a pressurized cooldown (McEligot and McCreery 2004). Approximate analyses have been applied to determine key nondimensional parameters and their magnitudes over this operating range. Flow is expected to be turbulent with momentum-dominated turbulent jets entering. An approximate analysis was conducted to estimate when a temperature gradient will stabilize a horizontal turbulent channel flow, thereby leading to reduced thermal transport near the upper surface. Initial conclusions are that (a) buoyancy influences are probably not important at full power, and (b) buoyancy is important at reduced power $(10 \%)$ at the side of the lower plenum away from the outlet, but not near the outlet. Thus, experiments without buoyancy effects will provide useful benchmark data for assessing CFD codes for some lower plenum flow conditions.

The purpose of fluid dynamics experiments is to develop benchmark databases for the assessment of CFD solutions of the momentum equations, scalar mixing, and turbulence models for typical VHTR plenum geometries in the limiting case of negligible buoyancy and constant fluid properties. The MIR facility and a sketch of the lower plenum model to be used in MIR are shown in Figure 28.

Using optical techniques in the MIR facility, such as particle tracking velocimeter (PIV), measurements can be obtained in the complex passages anticipated in the VHTR designs without disturbing the flow. The refractive indices of the fluid and the model are matched so that there is no optical distortion. The large size provides good spatial and temporal resolution. This facility has already been used to obtain velocity/turbulence data in scaled fuel channels for a VHTR concept (McCreery et al. 2003) and an SCWR concept (McEligot et al. 2003). 


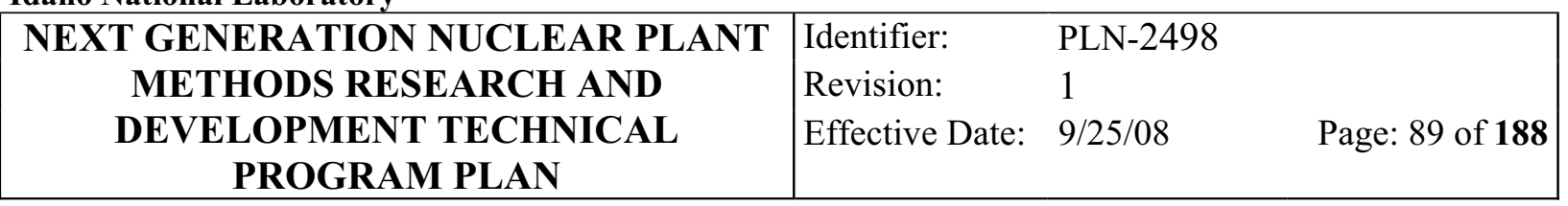

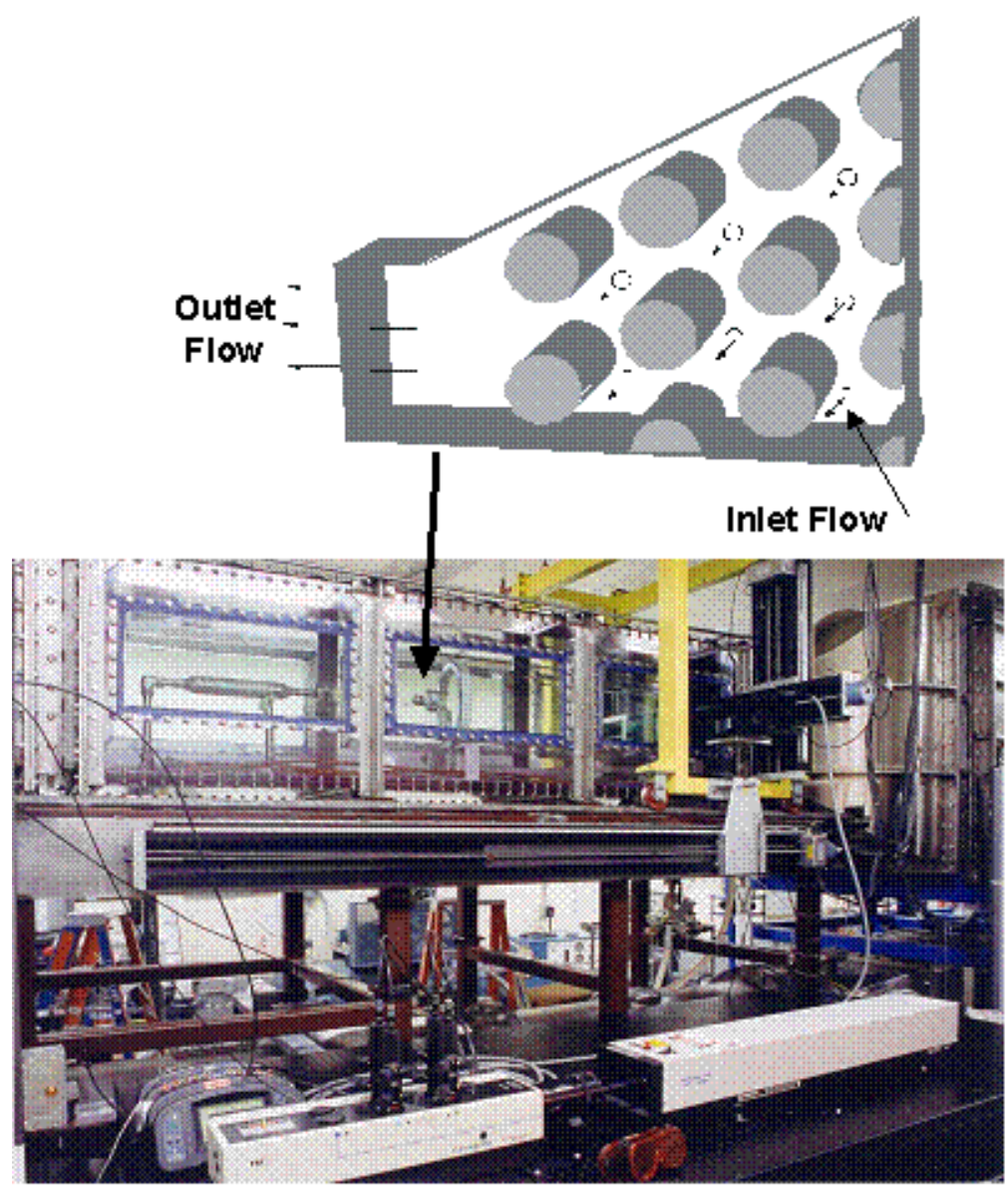

Figure 28. Matched-Index-of-Refraction flow system and a conceptual model design to study important flow features in a VHTR lower plenum.

McEligot and McCreery (2004) described the general characteristics of a typical VHTR lower plenum and, consequently, the features desired in idealized experiments aimed at assessment of CFD codes for VHTR applications. The first MIR lower plenum experiment will model flow across an array of posts (as near the outlet duct or in line with the duct centerline at the opposite side of the reactor) as indicated by near horizontal arrows in Figure 27. A plan view is shown in Figure 29 with open circles representing the locations of jet inlets and cross hatching indicating the support posts, approximately to scale. With crossflow from the right, flow is simulated from across the central region of the lower plenum (below a central reflector) into the last rows of jets from the active core. For the region furthest from the outlet, a solid wall can be inserted at the position indicated by the dashed line. In this case, flow would be completely provided by the jets as at the simulated location. Simulated plenum dimensions will be based on geometrical scaling of a current prismatic VHTR concept. The second MIR experiment will simulate flow aligned between a row of posts as suggested by the inclined arrow in Figure 27. 


\begin{tabular}{|c|lll|}
\hline NEXT GENERATION NUCLEAR PLANT & Identifier: & PLN-2498 \\
METHODS RESEARCH AND & Revision: & 1 & \\
DEVELOPMENT TECHNICAL & Effective Date: & $9 / 25 / 08$ & Page: 90 of 188 \\
PROGRAM PLAN & & & \\
\hline
\end{tabular}

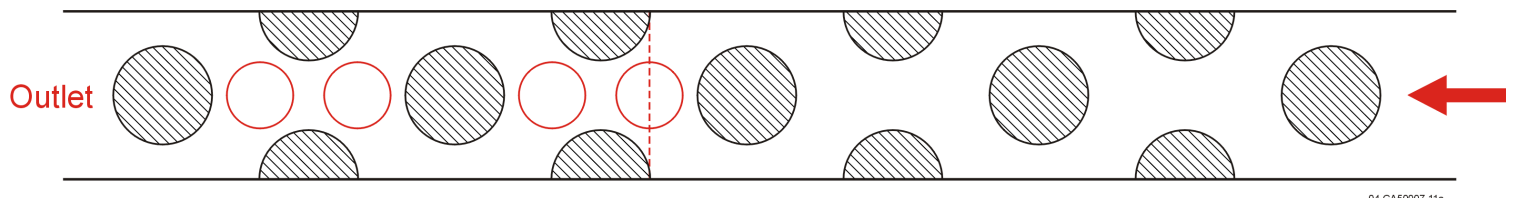

Figure 29. Conceptual design of model for the anticipated first MIR lower plenum experiment (plan view).

The experiments will be performed using a state-of-the-art particle imaging velocimetry (PIV) system installed during FY 2005. Measurements will be obtained with this PIV system by laser Doppler velocimetry (LDV) and possibly by 3-D particle tracking velocimetry (3D-PTV) with a moving particle tracking system. Both LDV, PIV, and PTV have advantages and may be considered as complementary approaches. Visualization of mean flows and instantaneous measurement of two velocity components should be available via the PIV system. For flow visualization and for measuring mixing, the PTV should be useful. LDV gives time-resolved measurements, but with PIV (and PTV) one needs many realizations to deduce means and the higher-order moments. The LDV technique is applied a point at a time; 3-D measurements are obtained by traversing (taking profiles) in three directions in turbulent flows that are steady in the mean. Provided the application can be considered to be at least quasi-steady (residence times quicker than other response times), useful data for assessment can be obtained from such steady flows. Typical results will include time-resolved, point-wise distributions of the mean velocities and their Reynolds stress components. The LDV time series will also be available for spectral and wavelet analysis to investigate potential shedding of eddies from the posts. Further background and information on this experiment are presented in the report by McEligot and McCreery (2004).

The first model (Figure 29) has been designed, fabricated, and installed in the MIR test section. Measurements began in late FY 2005. Although this test section is narrow, wall effects will be minimized by injecting jets from the side and by having a sufficiently large test section width to pitch ratio. Further measurements and documentation will be conducted during FY 2006 during which the second model will also be designed and fabricated. The key deliverables will be documented databases for CFD code assessment as described above. The approach of this experiment will also be employed for the later experiments on exit flows in pebble-beds, on geometric transitions of plenum inlets/outlets, and on prototypical geometries identified in the VHTR design and licensing phases.

\subsubsection{Heated Flows in Lower Plenums}

A follow-on experiment will treat heated (buoyant) jets entering a model which simulates key geometric aspects of a lower plenum; an apparatus with gas flow will likely be employed for this case. Preliminary analyses indicate that injection of buoyant jets could lead to thermal stratification of hot helium near the upper surface of the plenum in the region away from the outlet, under reduced power operations and possibly under full power. The objective of this experiment is to provide benchmark data to assess CFD codes that include treatment of buoyancy forces and gas property variation in predicting thermal mixing.

Two types of conceptual model designs are being considered, some using gas flow and some using water. Density differences between hot and cool jets in designs using gas flow are introduced by varying the gas temperature. Density differences in a water flow apparatus are simulated by varying the density of the injected water with the addition of soluble material, such as salt to water stored in a reservoir, before injection. A scaling analysis indicates that the two methods are equivalent for modeling buoyancy 


\begin{tabular}{|c|lll|}
\hline NEXT GENERATION NUCLEAR PLANT & Identifier: & PLN-2498 \\
METHODS RESEARCH AND & Revision: & 1 & \\
DEVELOPMENT TECHNICAL & Effective Date: & $9 / 25 / 08$ & Page: 91 of 188 \\
PROGRAM PLAN & & & \\
\hline
\end{tabular}

influences in lower plenum flow. Further details of various proposed concepts are discussed in McEligot and McCreery (2004).

A model concept for heated gas flow is shown as Figure 30. The posts and lower plenum walls are idealized as cylinders positioned between flat walls, somewhat like the plan view in Figure 29. The inlet flow channels, from which the buoyant jets are formed, are represented by tubes attached to the top wall. Hot and cold gases are injected through these tubes at flow rates and temperatures determined from scaling analyses. In this case, all flow in the simulated plenum is induced by the incoming jets similar to the region opposite the plenum outlet in a prismatic VHTR concept.

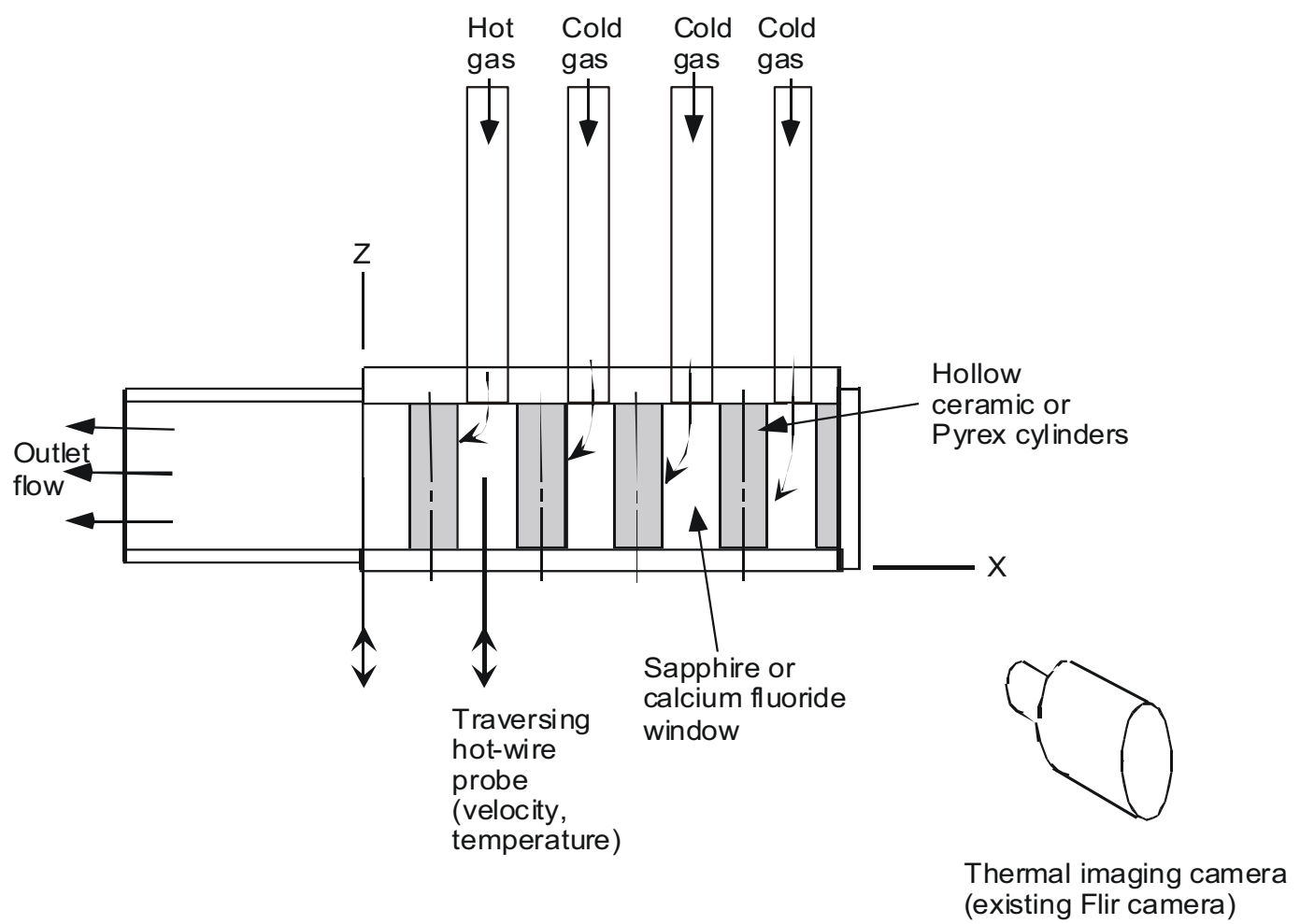

Figure 30. Schematic diagram of benchmark experiment to simulate effect of buoyancy on thermal mixing in a lower plenum.

The gas temperature field will be mapped using both a traversing thermal-sensor probe and a thermal imaging camera. The bottom wall of the apparatus will contain multiple access holes for installing probes in various positions. Local mean temperatures and temperature fluctuations will be measured using a traversing cold-wire probe (effectively a fast-response resistance thermometer). The front wall of the apparatus will consist of a sapphire window that will transmit visible and infrared light for reception in the thermal imaging camera. The camera will record the thermal images of the cylinder surfaces. The cylinder material will be a thin-wall ceramic or Pyrex. The thin walls minimize axial conduction heat transfer so that the surface temperature distribution will be approximately equal to the local mean gas temperature. Although maximum gas temperatures will be limited to approximately $500^{\circ} \mathrm{C}$ because of material temperature limits, this limitation poses no restriction of the validity of the validation data since the data will still be within the proper range of applicable nondimensional numbers. Additional measuring techniques under consideration include employing heat pipes to represent the support posts, embedding heat flux gages on the surfaces, and using naphthalene to apply the heat-mass transfer analogy to infer 


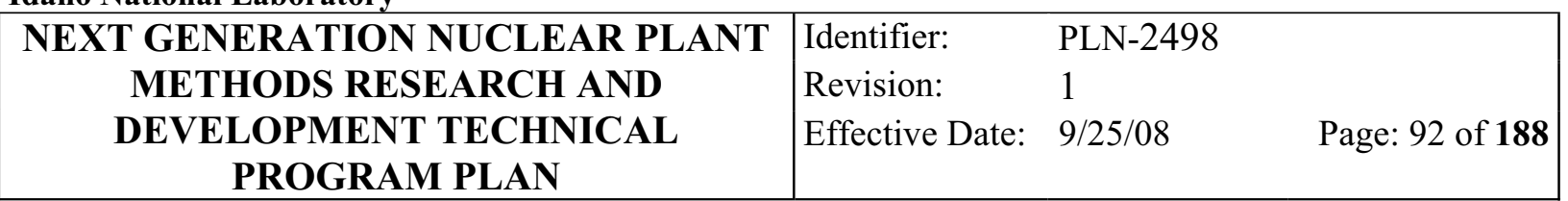

heat transfer coefficients (Rhee, Yoon, and Cho 2003; Angioletti et al. 2003). With the latter approach, assessment of CFD codes could be accomplished directly by comparing mass transfer predictions (solutions of species conservation equations) to the data.

The PIV system to be employed in the MIR experiments will also be ideal for mapping velocity fields in buoyant flow experiments with air (or water) flow. The PIV system may be extended to measure velocity and temperature fields (for use in the gas flow experiments) or velocity and concentration fields (for use in the water flow experiments) simultaneously. Velocity plus temperature (or concentration) may be measured by using fluorescent seeding particles and two appropriate cameras with narrow-band filters. One camera, with a filter centered on the laser wavelength, records the velocity information and the other acquires the fluorescence signal. The latter method, termed planar-laser-induced-fluorescence, relates signal strength to either temperature or concentration, depending on the seeding material. The capability of extending the PIV system to include planar-laser-induced-fluorescence for the buoyant mixing experiments will be evaluated during the acquisition of the PIV system.

The studies for FY 2005 and FY 2006 include finalizing the conceptual design of the competing experiments for examining buoyancy effects in a lower plenum, estimating costs and schedules for each, and selecting a path forward. Final design, fabrication and initiation of measurements are planned for FY 2007, with most measurements and documentation occurring in FY 2008 and FY 2009.

\subsubsection{Interactions Between Hot Plumes in an Upper Plenum and Parallel Flow Instabilities}

The mixing of hot plumes in the upper plenum of a gas-cooled reactor is of concern during a pressurized cooldown (McEligot et al. 2002). These plumes come from up-flow in the hot coolant channels during natural circulation in the core and may impinge on the reactor vessel upper-plenum structure and control-rod apparatus causing localized hot spots that may be prone to failure. The flow rates and temperatures of the plumes may be affected by laminar flow instability caused by variations in the viscosity with temperature (Reshotko 1967) at the low Reynolds numbers in these channels and possibly cause flow choking. A planned experiment on interactions between hot plumes and parallel flow instabilities will examine this problem.

The envisioned experiment will produce a scaled fluid behavior simulation of plumes moving upwards from the hot core cooling channels of the natural circulation development in the upper plenum and of the downward movement of upper plenum inventory into the cooler channels in route to the lower plenum. Sufficient instrumentation will be used to characterize the flow behavior for CFD validation data sets.

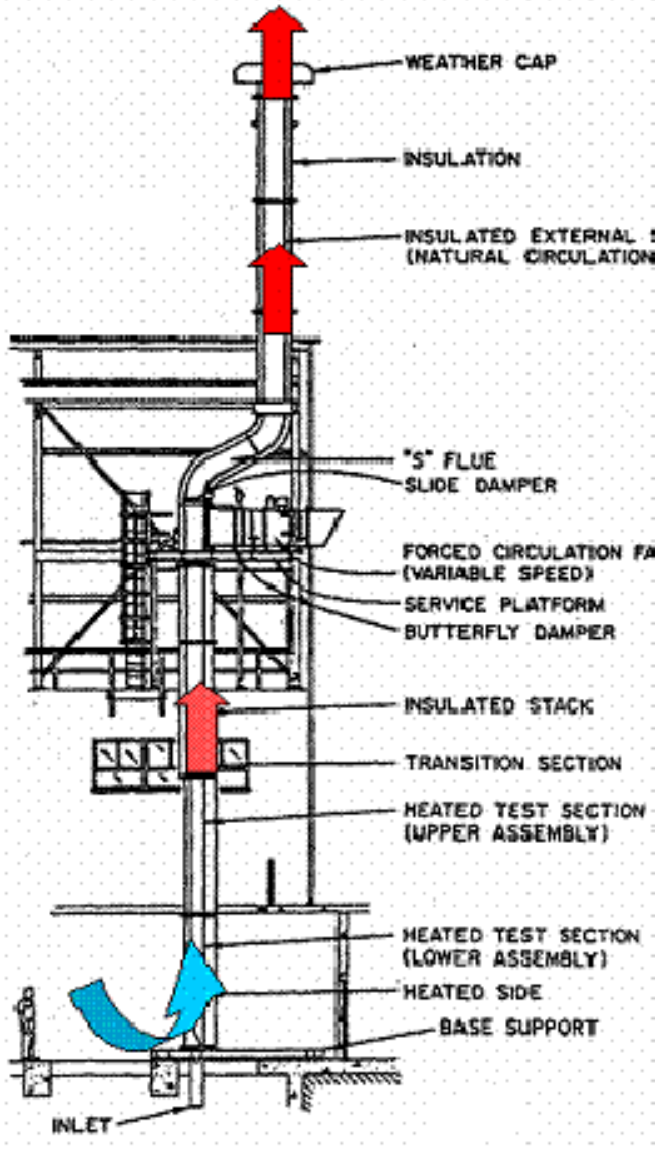

Figure 31. Schematic of ANL Natural Convection Shutdown Heat Removal Test Facility. 


\begin{tabular}{|c|lll|}
\hline NEXT GENERATION NUCLEAR PLANT & Identifier: & PLN-2498 \\
METHODS RESEARCH AND & Revision: & 1 & \\
DEVELOPMENT TECHNICAL & Effective Date: & $9 / 25 / 08$ & Page: 93 of 188 \\
PROGRAM PLAN & & & \\
\hline
\end{tabular}

\subsubsection{Reactor Cavity Cooling System Experiments}

Research on the RCCS is essential since the heat transfer from the reactor pressure vessel to the RCCS is a key ingredient in defining the peak core and vessel wall temperatures during postulated accident conditions. Two RCCS experimental efforts are presently underway. The first, at ANL, aims to characterize the heat removal capabilities of both an air-cooled RCCS and a water-cooled RCCS. The second, at Seoul National University (SNU), aims to characterize the heat removal capabilities of a watercooled RCCS.

\subsubsection{ANL RCCS Task}

The objective of this task is to acquire the model/code validation data for natural convection and radiation heat transfer in the reactor cavity and the RCCS by performing experiments in the ANL Natural Convection Shutdown Heat Removal Test Facility (NSTF). The NSTF will be used as a experiment simulator (see Figure 31). The first step will be to determine scalability of the existing data and configuration of the ANL RCCS simulator to the RCCS designs. The scaling studies will identify the important nondimensional parameters for each separate-effects study for both air-cooled and water-cooled systems. Based on the results of the scaling/feasibility study, the range of experiment conditions will be determined as will the appropriate experiment scale and appropriate fluids to be used that most effectively simulate full-scale system behavior. R\&D will include the identification of RCCS design candidates from both the pebble-bed and prismatic options. The range of thermal-hydraulic conditions for normal operating and accident events will be evaluated. An instrumentation strategy will be developed to ensure that adequately detailed velocity and turbulence profiles and surface pressure and/or temperature distributions are obtained for the validation of multidimensional simulation tools. Based on the results of these scaling/feasibility studies and the RCCS analyses carried out in Subsection 6.2.3, a detailed engineering modification plan for the ANL RCCS facility will be developed. Next, a test matrix will be developed and the indicated test program will be performed. The ANL RCCS experimental results will capture key phenomena expected to be present in the RCCS and provide data of sufficient resolution for development and assessment of applicable CFD (STAR-CD/Fluent) and system codes (RELAP53D/ATHENA). Both air and water-cooled RCCSs will be included in the NSTF test plan.

In more detail, the workscope for the modification of the NTSF to perform experiments for the VHTR RCCS has been divided into the nine tasks described below. These tasks represent the work required to achieve completion of Phase 1 as described in the introduction of this section - the R\&D required prior to selection of the specific NGNP design:

1. The first task is to obtain the necessary laboratory ES\&H approvals to initiate the work. This includes preparing and submitting National Environmental Protection Act (NEPA) documentation and a safety plan for covering the laboratory's efforts. The key deliverables from this task are DOE and laboratory approvals to initiate testing in the facility.

2. Task 2 consists of preparing a Quality Assurance (QA) plan for the program. This plan will be developed in close collaboration with INL to ensure that the results produced by the project will meet end-user needs and requirements. The key deliverable from this stage of the work will be a Program QA Plan approved by both INL and ANL laboratory and project management.

3. Task 3 consists of developing a facility test plan, which covers the development of final designs for the facility mechanical, electrical, instrumentation, data acquisition, and control systems, as well as the supporting fabrication drawings and equipment specifications required for procurement. From the mechanical viewpoint, this includes the fabrication of both instrumented and dummy RCCS tubes and the tube support and bracing structure within NSTF. The instrumentation plan for measuring flow 


\begin{tabular}{|c|lll|}
\hline NEXT GENERATION NUCLEAR PLANT & Identifier: & PLN-2498 \\
METHODS RESEARCH AND & Revision: & 1 & \\
DEVELOPMENT TECHNICAL & Effective Date: & $9 / 25 / 08$ & Page: 94 of 188 \\
PROGRAM PLAN & & & \\
\hline
\end{tabular}

rates, temperatures, pressures, surface emissivities, and turbulence characteristics at key locations throughout the system will be finalized. The design and specifications of the electrical system for controlling and monitoring the duct wall heaters will also be completed. Finally, general operating procedures for the facility, from cold shakedown testing to hot startup, will be developed. The key deliverable from this stage of the work will be an approved facility test plan.

4. Task 4 covers the key area of experiment planning. Laboratory personnel will collaborate with analysts and data end-users at INL and ANL to identify key RCCS conditions and data ranges expected during both PCC and DCC event sequences for potential VHTR air-RCCS designs. Based on this input, a test matrix and its companion facility operating procedures will be developed that will generate the data needed for code $\mathrm{V} \& \mathrm{~V}$. The key deliverable from this task will be an experiment test matrix planning report.

The balance of the tasks cover the laboratory efforts required to bring the facility up to an operational stage and to begin conducting tests identified under Task 4. Progress during these various stages of work will be documented in the form of monthly progress reports to the sponsor.

5. Task 5 covers preparation of the laboratory space where the facility resides. The high-bay area will be prepared and surplus items will be disposed of. Similar preparation and repair activities will be conducted in the facility control room under current Operational Safety and Health Administration standards.

6. Once the lab space is updated, Task 6 will be initiated to modify the facility. This task includes the procurement of services and equipment for conducting the work.

7. Task 7 consists of revisions to the facility itself. The key steps in the mechanical modifications to the facility consist of removal of insulation from the two heated test sections to facilitate work; removal of test section nonheated duct wall plates and disposal of the existing inlet plenum; machining of the nonheated plates to receive new instruments for flow characterization and modifications to the test assembly to gain access for the tube upper support plate; manufacture, instrumentation, and installation of the RCCS tubes; installation of stud extenders and insulation to expand channel width and the modified nonheated duct wall plates; checkout of all thermocouples and heaters after reassembly; and installation of the lower tube guide plate and the new inlet plenum ductwork. With these steps completed, instruments will be installed and wired into the Data Acquisition System (DAS) according to the Test Plan. In parallel with this work, the test section heaters will be wired to the heater temperature controllers, relays, and contactors. The associated power monitoring instruments will be wired into the DAS. This work will include rewiring the heater power feeds from the control room into the relay racks in the high bay.

8. With these steps completed, Task 8 , facility shakedown testing, will be initiated. This work will include checkout of the controllers and heaters for proper operations under low-power conditions (heater temperatures $<150^{\circ} \mathrm{C}$ ) and checkout of flow measurement devices at room temperature using the fan loft to provide test section $\Delta \mathrm{P}$. After these checkouts are completed, the facility will be brought up to conduct initial low power testing (heater temperatures $\sim 150^{\circ} \mathrm{C}$ ) to verify proper integral operation.

9. With these steps completed, Step 9 will initiate actual data generation in accordance with the test matrix developed under Task 4 of the overall work scope.

During the predesign selection phase, the feasibility study for the water-cooled RCCS option will be updated based on the calculations from Section 6.1.1 carried out to confirm the scaling analyses of FY 2006. Some of the nine steps listed above will also be carried out, including Steps 1, 2, 5, and parts of 7 . 


\begin{tabular}{|c|lll|}
\hline NEXT GENERATION NUCLEAR PLANT & Identifier: & PLN-2498 \\
METHODS RESEARCH AND & Revision: & 1 & \\
DEVELOPMENT TECHNICAL & Effective Date: & $9 / 25 / 08$ & Page: 95 of 188 \\
PROGRAM PLAN & & & \\
\hline
\end{tabular}

\subsubsection{Seoul National University Water-Cooled RCCS Experiment}

A water-cooled RCCS design may be preferred because its heat removal capability has a larger per-unit heat transfer area than a comparable air-cooled design. Hence, a water-cooled design would be more desirable if a high-pressure containment is required for the VHTR instead of a low-pressure confinement system.

The SNU RCCS facility consists of three parts: the reactor vessel, an air cavity, and a water pool (see Figure 32). The SNU experiments are being performed using various gas mixtures in the gap and with various water pool elevations. The temperatures on the various surfaces are measured together with the surface emissivities and water pool characteristics (temperature as a function of position, elevation, etc.). Heat from the reactor vessel is transferred to the RCCS by radiation, natural convection, and conduction. The data provided by these experiments are the basis for validation CFD calculations specific to the behavior of water-cooled RCCS.

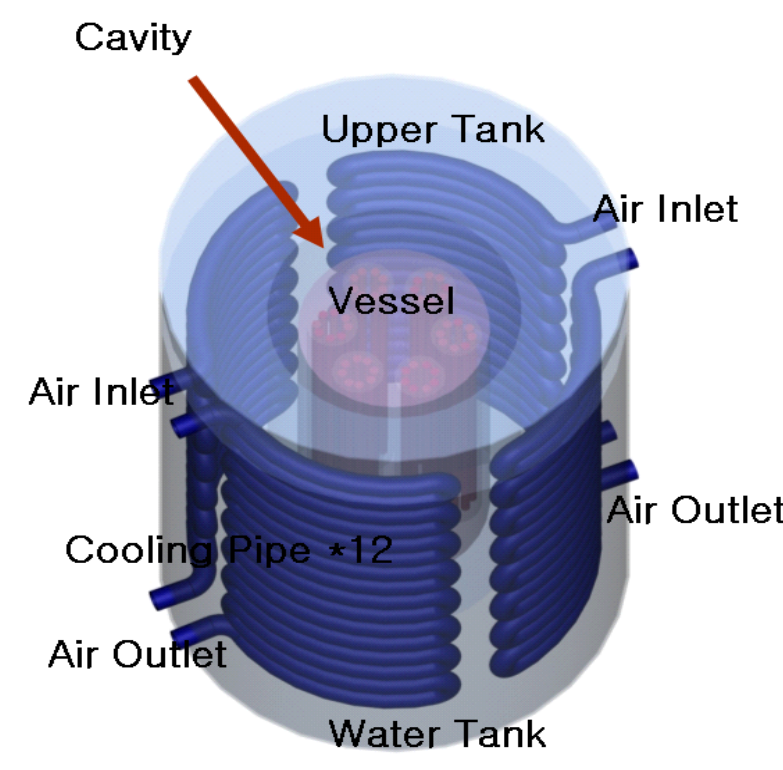

a. Schematic of test facility.

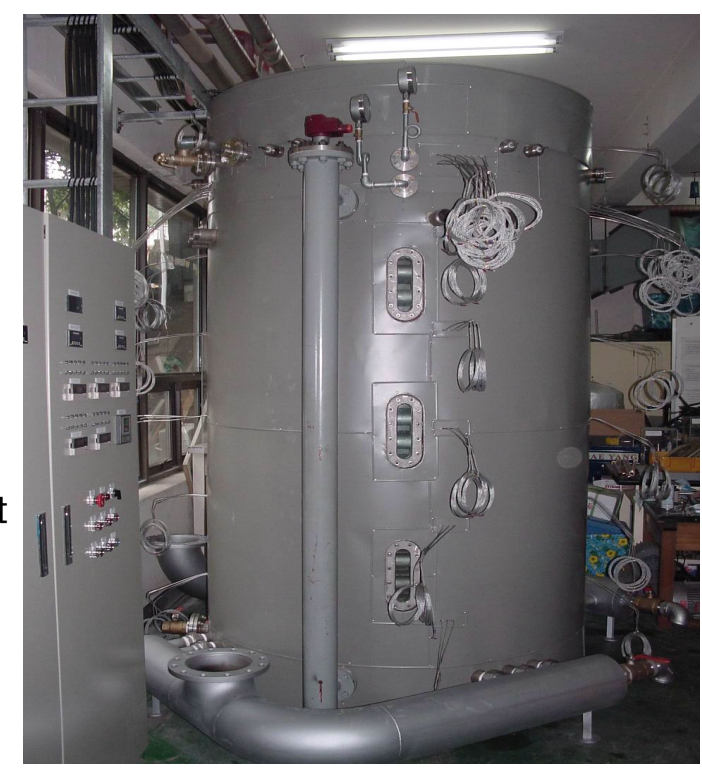

b. Photograph of test facility.

Figure 32. SNU water-cooled RCCS experiment.

\subsubsection{Air Ingress-Related Experiments}

\subsubsection{Stratified Flow Experiments}

Subsequent to the break in the hot duct hypothesized in depressurized conduction cooldown, air present in the reactor cavity will enter the reactor vessel via stratified flow. Because of the significantly higher molecular weight and lower initial temperature of the reactor cavity air, the air-helium mixture in the cavity is always heavier than the helium discharging through the break. In the later stages of the helium blowdown, the momentum of the helium flow decreases sufficiently for the heavier cavity air to intrude into the reactor vessel lower plenum through the lower portion of the break. Once it has entered, the heavier gas will pool at the bottom of the lower plenum. From there, it will diffuse upwards into the core. This condition (based on the stratified flow assumption) is considerably different from the standard 


\begin{tabular}{|c|lll|}
\hline NEXT GENERATION NUCLEAR PLANT & Identifier: & PLN-2498 \\
METHODS RESEARCH AND & Revision: & 1 & \\
DEVELOPMENT TECHNICAL & Effective Date: & $9 / 25 / 08$ & Page: 96 of 188 \\
PROGRAM PLAN & & & \\
\hline
\end{tabular}

assumption used in calculations to date where the air is assumed to diffuse into the lower plenum via the break area itself. Significant differences between the standard assumption and the stratified flow assumption include (assuming stratified flow occurs through the break): (a) a much larger flow area (the full vessel cross-sectional area) is available for diffusion to occur assuming stratified flow occurs, (b) stratified flow is a much more rapid mechanism for moving air into the vessel leading to the presence of significant quantities of air in the lower plenum, and (c) a much shorter distance separates the air present in the lower plenum from the core if stratified flow occurs. Hence, the stratified flow assumption will likely lead to a more rapid air ingress into the core and also the presence of more air for core graphite oxidation than the standard assumption.

In essence, the stratified flow assumption is based on the formation of a wedge of air at the lower portion of the hot duct that will advance into the reactor vessel as a function of the density-gradients when the blowdown has become unchoked. Such flows are well characterized by the densimetric Froude number that correlates the densities of helium and the air mixture to a constant value representative of the flow condition at different times in the scenario.

For $\mathrm{d}=$ centroidal distance of the of the upper and lower half of the orifice area from the orifice center, and

$$
g^{\prime}=\frac{g\left(\rho_{2}-\rho_{1}\right)}{\frac{\rho_{2}+\rho_{1}}{2}}
$$

which can be viewed as a reduced gravitational acceleration because of the buoyancy effect induced by the density difference, the densimetric Froude number is

$$
F=\frac{u}{\sqrt{g^{\prime} d}}
$$

where $\mathrm{F}$ is known as a densimetric Froude number and $\mathrm{u}$ is the air velocity. The above idea and experimental confirmation can be found in Yih (1980).

A stratified flow experiment is required to better understand the phenomenon, provide data for CFD code validation, and develop correlations for 1-D system codes such as RELAP5 to model this inherently multidimensional phenomenon. It is expected the densimetric Froude number will be found to be a function of

$$
F=f\left(\alpha, L / D, \frac{V_{\text {vessel }}}{V_{\text {vault }}}, P_{R}, R\right)
$$

in which $\alpha=$ orientation of the break with respect to the vertical, $\mathrm{L}=$ length of the separated hot duct on the reactor vessel side, $\mathrm{D}=$ diameter of the hot duct, $\mathrm{V}=$ volume, $\mathrm{Pr}=$ Pressure coefficient, and $\mathrm{R}=$ Reynolds number.

\subsubsection{Heat Transfer and Wall Friction of Air-Helium Mixtures}

Eexperiments are planned during FY 2007 and FY 2008 to provide data required to accurately treat heat transfer and wall friction of air-helium mixtures during air ingress events. For accident scenarios 


\begin{tabular}{|c|lll|}
\hline NEXT GENERATION NUCLEAR PLANT & Identifier: & PLN-2498 \\
METHODS RESEARCH AND & Revision: & 1 & \\
DEVELOPMENT TECHNICAL & Effective Date: & $9 / 25 / 08$ & Page: 97 of 188 \\
PROGRAM PLAN & & & \\
\hline
\end{tabular}

involving air ingress, the heavier air will displace the helium used as the normal working fluid. However, a consequence of this displacement process will be a mixture of the two gases in some components. As the concentration of a gas mixture changes, so do its properties. It is known that the variation of thermal conductivity and specific heat versus concentration will lead to a minimum in the Prandtl number at an intermediate concentration, where the Prandtl number realizes a value between that for liquid metals and common gases. Thus the applicability of typical correlations and turbulence models in these cases are open to question. Taylor, Bauer, and McEligot (1988) have shown that some of the most popular correlations over predict convective heat transfer for other binary gas mixtures at high Reynolds numbers. Still, data are needed for forced and mixed convection in low-Reynolds-number turbulent flows. Graphite oxidation data are available from other sources (Schultz, Ball, and King 2004).

The objective of this simple experiment will be to obtain benchmark data for the preliminary assessment of CFD turbulence models and systems codes for forced and mixed convection in lowReynolds-number turbulent flows occurring during air ingress or resulting natural circulation. Wall temperatures and pressure drops will be measured for specified wall heat flux and inlet conditions with apparatus comparable to that employed by Taylor, Bauer and McEligot (1988) but modified to achieve lower relative heat losses at the lower Reynolds numbers. Experimental procedures employed will also correspond to those of Taylor, Bauer and McEligot (1988). System pressure and test section diameter will be adjusted to provide the necessary range of nondimensional parameters. With these data, turbulence models may be evaluated by comparison of wall temperature and static pressure distributions. Figure 24 shows an example of this type of comparison.

\subsubsection{Larger-Scale Vessel Experiments and Prototypical Concept Experiments}

Code development and assessment activities for other reactor designs have required integral experiments at various scales to verify that small-scale laboratory experiments, experiments using simulated fluids, and experiments at nonrated conditions have been properly scaled for the full-scale plant. This premise also holds true for any VHTR design.

Two approaches will be used to obtain applicable integral facility data to validate the software for the NGNP VHTR. The first will be to use existing data from the HTTR and/or the HTR-10 research reactors. It is hoped that via agreements with DOE, experiments that are specific to the NGNP may be defined in at least one of these test reactors. The second will be to build integral facilities based on need. These subjects are addressed in the subsequent subsections.

\subsubsection{Integral Reactor Experiments-HTR-10 and HTTR}

Presently there are two operational gas-cooled test reactors: the HTR-10 and the HTTR. These experiments are located in Beijing, China at the Institute of Nuclear Energy Technology (INET), and in Oarai, Japan, at JAEA, respectively. Integral experiments are the only experimental sources that may be able to produce the complex interactions between dominant phenomena identified in the VHTR system specific PIRT. Therefore, the integral experiments are essential for systems analysis and CFD code validation studies. Undoubtedly, data from both the HTR-10 and HTTR will be included in the calculational matrix required for plant licensing by the NRC. Validation studies are needed using the data generated at these facilities to date. In addition, arrangements will be made to enable the VHTR Program to collaborate with INET and JAEA such that specific experiments may be specified that can be linked directly to the VHTR preliminary and final design PIRTs. 


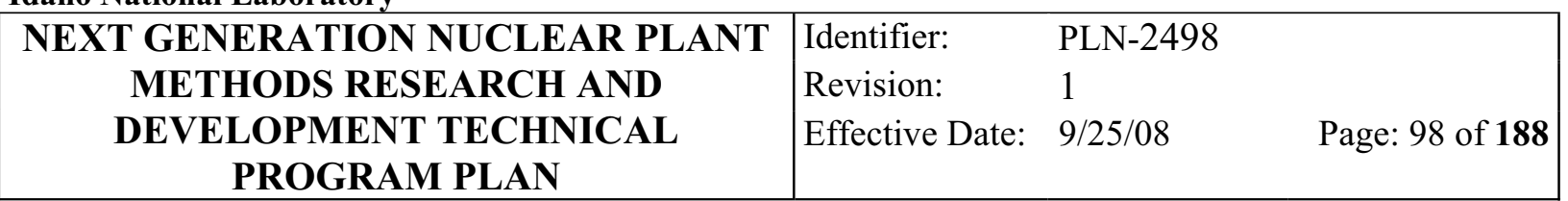

\subsubsection{HTR-10}

The HTR-10 is a $10 \mathrm{MW}$ pebble-bed high temperature gas-cooled reactor that became operational in 2000. INET plans to perform a spectrum of experiments of essential to the VHTR Project. Among the experiments may be a LOCA, a pressurized conduction cooldown experiment, a rod ejection experiment, and an anticipated transient without scram.

The HTR-10 reactor vessel (see Figure 33) is approximately $11.2 \mathrm{~m}$ high and contains a 1.8 -m-diameter core that is $1.97 \mathrm{~m}$ high with $\sim 27,000$ pebbles. The reactor was designed to operate at $10 \mathrm{MWt}$, but the average power density is $2 \mathrm{MW} / \mathrm{m}^{3}$. The core inlet temperature is 250 to $300^{\circ} \mathrm{C}$ and the core outlet temperature will range from 700 to $900^{\circ} \mathrm{C}$. Benchmark experiments performed in the HTR-10 are available via the International Atomic Energy Agency (IAEA) (Sun and Gao 2003).

\subsubsection{HTTR}

The HTTR Project is centered on the $30 \mathrm{MWt}$ prismatic engineering test reactor (see Figure 34). However, the HTTR Project also has a number of support projects that provide useful data (e.g., the Vessel Cooling System test series based on cooling panels inside a vessel containing heating elements and the heat transfer studies based on the hemispheres heated from below and cooled using natural convection). JAEA plans, to perform a spectrum of HTTR experiments that may include a LOCA, a pressurized conduction cooldown experiment, a rod ejection experiment, and an anticipated transient without scram.

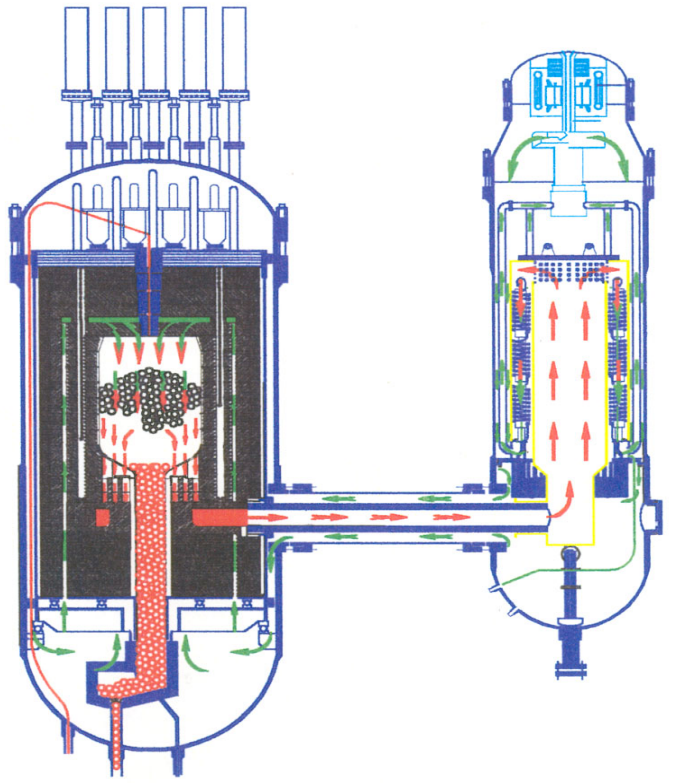

Figure 33. Schematic of HTR-10 (from HTGR Proceedings, Beijing, China, March 2001).

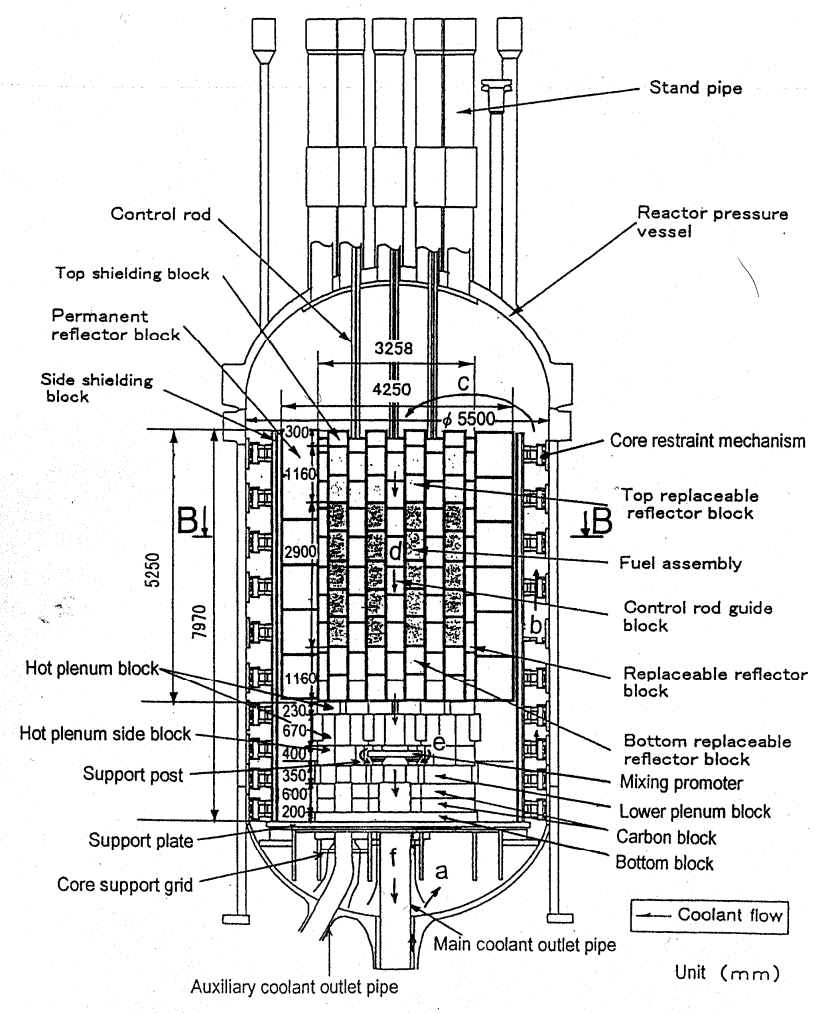

Figure 34. Schematic of HTTR. 


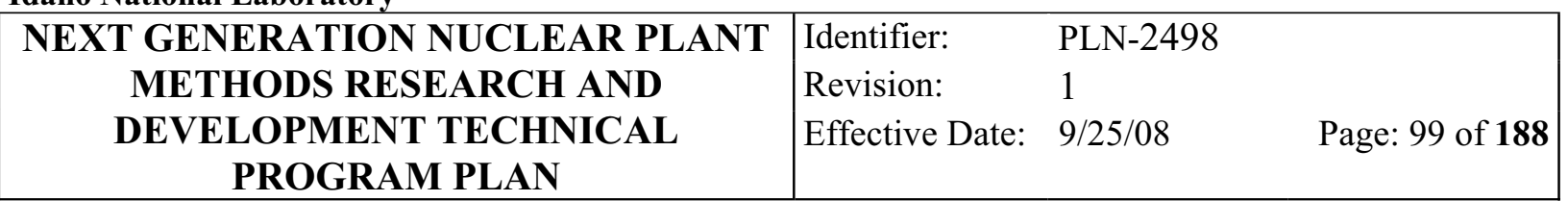

The HTTR became operational in 1998. The reactor vessel is $13.2 \mathrm{~m}$ tall (inner dimension) and has a $5.5 \mathrm{~m}$ inner diameter. The core has 30 fuel columns and seven control rod guide columns. There are 12 replaceable reflector columns and nine control rod guide columns. The HTTR is fitted with an RCCS. The HTTR operates at $4 \mathrm{MPa}$ with a core inlet temperature of $395^{\circ} \mathrm{C}$ and outlet temperature of $850^{\circ} \mathrm{C}$ (Sikusa 2000). However, it is known that the HTTR does not have a full set of instrumentation. Thus, additional instrumentation is required to obtain the needed data.

Supporting experiments include a series of six tests performed to simulate the heat transfer to the RCCS cooling panels (IAEA 2000). The experiments are summarized in Table 7.

Table 7. RCCS Experiments: HTTR Project

\begin{tabular}{|llllllll|}
\hline \multicolumn{1}{|c}{ Experiment } & \multicolumn{1}{c}{ I } & \multicolumn{1}{c}{ II } & \multicolumn{1}{c}{ III } & \multicolumn{1}{c}{ IV } & \multicolumn{1}{c|}{ V } & \multicolumn{1}{c|}{ VIa } & \multicolumn{1}{c|}{ VIb } \\
\hline Gas & Vacuum & helium & nitrogen & helium & helium & helium & helium \\
Pressure (MPa) & $1.3 \times 10-6$ & 0.7 & 1.1 & 0.47 & 0.64 & 0.96 & 0.98 \\
Power $(\mathrm{kW})$ & 13.1 & 28.8 & 93.9 & 77.5 & 29.7 & 2.6 & 8.0 \\
Cooling panel & water & water & water & water & air & air & air \\
\hline
\end{tabular}

Cooling panels were placed inside a pressure vessel and experiments were performed by varying the gas in the pressure vessel to change the natural convection characteristics; thus Experiment I was performed with a vacuum so no natural convection would occur and the only heat transfer from the heaters to the cooling panels would be radiation. Experiment III was performed with nitrogen and the remainder of the experiments were performed using helium. Also the cooling medium in the cooling panels was water in four experiments and air in three experiments. The power level was changed as shown.

A further example of the types of experiments performed in the HTTR include a series of experiments making use of a $0.3 \mathrm{~m}$ hemisphere that was heated from below, while the natural circulation characteristics were measured. This experiment was designed to provide validation data relevant to calculating natural circulation in passive systems for CFD and systems analysis software.

\subsubsection{Integral Facilities}

While some integral data may become available from the HTTR and the HTR-10 research reactors, there will undoubtedly be a data gap when considering measurements needed to validate calculations from coupled CFD/systems codes for the final VHTR reactor geometry. Therefore, a larger scaled vessel experiment will be performed to provide scaled data directly applicable to the final VHTR design and to quantify potential distortions from data of small-scale facilities not apparent in the scaling studies.

The studies planned for FY 2009 to FY 2011 are experiments that are expected to be necessary during the development of the VHTR and of its CFD analyses for design, safety, and licensing. Most will depend on details still to evolve in the selection and design phases, such as the choice between prismatic and pebble-bed approaches, and should be decided by undergoing a study specifically aimed at designing the experiment(s). New thermal fluid issues will be identified as the VHTR development proceeds through conceptual, preliminary, and final design stages and then construction, licensing, and operation (FY 2007 to FY 2012). While the needs may be forecast now, the scaling studies and conceptual experiment designs await the details of the VHTR designs.

During the period FY 2009 through FY 2012 near-prototypical heat transfer and fluid mechanics experiments will be developed and conducted to treat geometries and issues identified as competing 


\begin{tabular}{|c|lll|}
\hline NEXT GENERATION NUCLEAR PLANT & Identifier: & PLN-2498 \\
METHODS RESEARCH AND & Revision: & 1 & \\
DEVELOPMENT TECHNICAL & Effective Date: & $9 / 25 / 08$ & Page: 100 of 188 \\
PROGRAM PLAN & & & \\
\hline
\end{tabular}

concepts evolve. These experiments and the related CFD codes should be valuable in quantifying key thermal fluid behavior to assist in the selection process with independent safety analyses. In general, the techniques used for these experiments will be comparable to those employed in the earlier years. For example, the future fluid dynamics experiments will be by nondisturbing optical measurements as in the INL MIR flow system and gas flow data for pressure distributions; the heat transfer studies will use the approaches in the examination of heated flows in the lower plenum but at larger scales, in some cases.

A highly instrumented, geometrically correct, larger-scale simulator will be constructed consisting of a VHTR upper plenum, core simulator, lower plenum, hot outlet duct, and turbine inlet channel. Geometry will be defined by the best available information on the actual design. The scale required will be determined from previous experimental results and available literature and from phenomena expected to occur. Size on the order of $1 / 4$ to $1 / 3$-scale is envisioned, with lower pressures and temperatures than design targets. The core simulator may or may not be electrically heated. Overall instrumentation will be sufficient to provide detailed local data for CFD code assessment, as well as global data for systems code assessment. The facility will be capable of simulating both operational conditions and accident scenarios. Issues that can be studied for operational conditions include the influence of various bypass conditions on the system operational envelope, the progression of mixing and turbulence of the helium as it passes from the lower plenum through the hot outlet duct to the turbine inlet, and the influence of various lower plenum configurations on the system performance. Accident conditions that can be examined include the influence of natural circulation on the thermal behavior of the system during depressurized cooldown.

\subsection{Introduction to Thermal-Fluids Design Methods Development, Validation, and Analysis}

The thermal-hydraulics design, performance analysis, and ultimately the licensing of the VHTR will require the use of validated computer codes for modeling the reactor's behavior during normal operation, anticipated transients, and accident conditions. The modeling strategy chosen for this effort is to make use of both thermal-hydraulic systems analysis codes and CFD codes. The reference codes chosen are the RELAP5-3D systems code and the Fluent and STAR-CD CFD codes. ${ }^{\mathrm{n}}$ However, other codes such as GRSAC, Abaqus, and NPHASE will be used to supplement the reference codes.

A systems analysis code is needed to model the integrated behavior of the entire VHTR system, including the interactive coupling of the reactor with the hydrogen and power producing components, including the intermediate heat exchanger, turbine, compressor, reheaters, etc.

CFD software is needed to analyze or qualify simulated fluid behavior wherever 2-D or 3-D fluid behavior is expected, particularly in plenums and cavities. Regions of applicability for the VHTR include the upper and lower plenums, the hot duct, and the intermediate heat exchanger or turbine inlet region as well as the RCCS. Other VHTR regions will be identified during the upcoming PIRT studies.

n. RELAP5-3D includes all the working fluids presently being considered for the NGNP together with their associated constitutive models. Fluent is a commercial CFD code that was selected on the basis of its extensive usage and validation history and the commitment of its vendor to the NGNP program. During FY 2002 through FY 2004 a coupling capability was implemented to link RELAP5-3D models to a Fluent model. Fluent is being used by INL and STAR-CD is being used by ANL. 


\begin{tabular}{|c|c|c|c|}
\hline NEXT GENERATION NUCLEAR PLANT & Identifier: & PLN-2498 & \\
\hline METHODS RESEARCH AND & Revision: & 1 & \\
\hline $\begin{array}{l}\text { DEVELOPMENT TECHNICAL } \\
\text { PROGRAM PLAN }\end{array}$ & Effective Date: & $9 / 25 / 08$ & Page: 101 of $\mathbf{1 8 8}$ \\
\hline
\end{tabular}

\subsubsection{Methodology for Determining the Computational Fluid Dynamics Software}

Although two commercial CFD reference codes (Fluent and STAR-CD) are presently being used and a university-developed ${ }^{\mathrm{o}}$ or a national laboratory-developed ${ }^{\mathrm{p}}$ CFD code may also be used, it is suspected that none if them will meet all of the VHTR analysis requirements, thus requiring some modifications. A three-track approach will therefore be used to meet the CFD analysis needs for the VHTR:

- Track 1. Validation of currently available CFD software

- Track 2. Modification of existing tools as necessary

- Track 3. Pursuit of R\&D to obtain more efficient and effective simulation tools that may take several years to mature.

The near-term thermal-hydraulics tasks follow the first track-validating existing tools. As the CFD tools are validated, it may become necessary to add new turbulence models or pursue other modeling strategies such as Lattice-Boltzmann, LES, or DNS, thus following Track 2. Track 3 is designed to ensure that needed simulation tools that are more efficient and capable than existing tools will be available in the future. This approach is outlined in Figure 35.

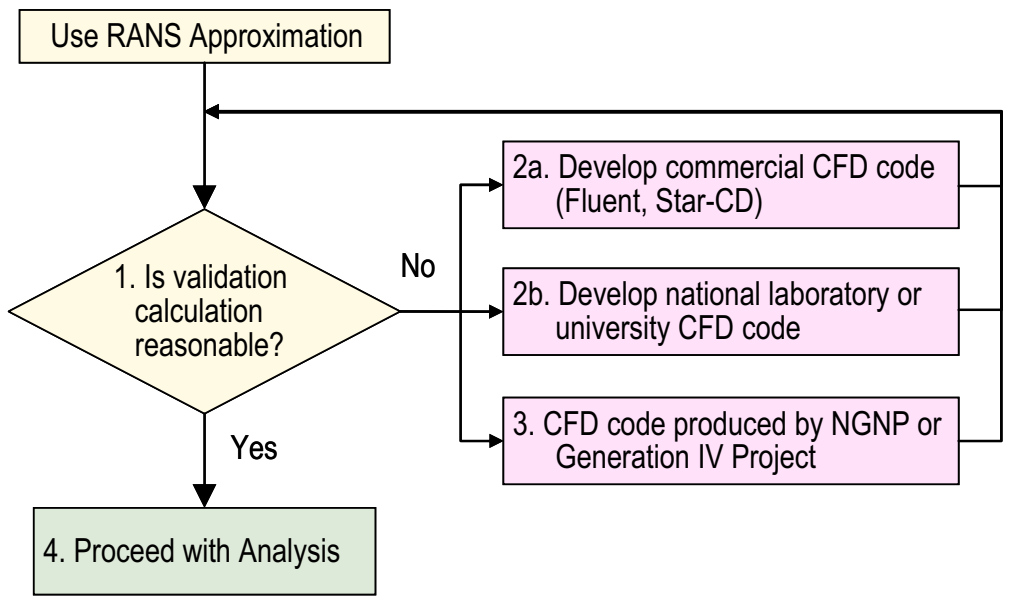

Figure 35. Approach for achieving validation objectives for CFD

Following the strategy outlined in the above two paragraphs, the commercial CFD codes Fluent (at INL) and STAR-CD (at ANL) will initially be validated and developed for the near-term thermalhydraulics tasks (Tracks 1 and 2). As deficiencies are isolated that cannot be addressed using the commercial CFD codes, experimental CFD software such as NPHASE and CFDLib will be tested and used to analyze the more difficult tasks.

o. An advanced next-generation CFD solver for both single-phase and multiphase flows developed at Rensselaer Polytechnic Institute. A new upgraded version of this code is currently under development under sponsorship of the U.S. Nuclear Regulatory Commission for future reactor safety analyses of next-generation reactors [Antal et al 2000].

p. CFDLib is the Los Alamos Computational Fluid Dynamics Library. This is a collection of codes. The CFDLib collection is a repository for all the numerical methodologies developed in the Fluid Dynamics Group (T3) of LANL's Theoretical Division. For example, the MAC method (due to Harlow and Welch), the ALE method (Hirt et al.), the multifluid ICE method (Harlow and Amsden), and the FLIP method (Brackbill and Ruppel) are all schemes that reside in the CFDLib collection. In recent years the CFDLib collection has been made into a sort of "open-source" project, with contributors from all over the academic world as well as many other divisions of LANL and other U.S. National Laboratories. For multiphase flow, the original capabilities of K-FIX (Rivard and Torrey) are contained in CFDLib [Kashiwa et al. 1993, 1994]. 


\begin{tabular}{|c|lll|}
\hline NEXT GENERATION NUCLEAR PLANT & Identifier: & PLN-2498 \\
METHODS RESEARCH AND & Revision: & 1 & \\
DEVELOPMENT TECHNICAL & Effective Date: & $9 / 25 / 08$ & Page: 102 of 188 \\
PROGRAM PLAN & & & \\
\hline
\end{tabular}

Because the spectrum of turbulent mixing behaviors that will be present in the plenums and flow passages are key phenomena that require evaluation, a significant research effort is aimed at identifying the proper model and approach. One of the more common approaches is based on taking an average of the incompressible Navier-Stokes equation to obtain the Reynolds-Averaged Navier-Stokes equations (RANS) as described by Speziale and So (1998). A newer approach toward predicting the ensemble average of the fluid velocity is LES “.... in which the Navier-Stokes equations are 'filtered' instead of averaged. This generates equations for the large flow scales yet uses a 'subgrid' model to capture the effect of the smaller scales" (Bernard et al. 1998, p. 13-3). Finally, the DNS approach of representing the Navier-Stokes equations enables all turbulence scales to be resolved.

\subsubsection{Potential Advanced CFD Development R\&D}

Three R\&D areas may be studied in the near future due to commercial CFD software that use some approaches that have been shown to potentially have excessive calculational uncertainties. These R\&D areas include (a) density driven flows with high heat flux (large temperature difference), (b) heat transfer in pebble-beds and at the PBR core/reflector boundary, and (c) graphite dimensional changes in irradiation and temperature fields. $\mathrm{R} \& \mathrm{D}$ is also required of specialized studies aimed at using variable mesh sizes in comprehensive models designed to capture the key phenomena while decreasing the compute time in conjunction with massively parallel systems. Each of these topics is briefly addressed below.

Density-driven flows with high heat flux. Most of the CFD simulations have treated helium as an incompressible flow with the addition of the Boussinesq approximation. ${ }^{\mathrm{q}}$ Incompressible flow assumes that density is constant in the problem. This will not be the case in helium with significant temperature variations. The question is the effect of this assumption on the total calculational uncertainty. Heat transfer rates at walls do vary between the Boussinesq approximation and the compressible NavierStokes. How large this difference might be in prototypic NGNP settings in the upper or lower plenum is still an open question.

Heat transfer in pebble-beds and at the core-reflector boundary. Present modeling techniques may not be adequate to properly represent the complex localized behavior that characterizes the flow in the region of hot spots in a pebble-bed core. Consequently, focused studes may be required to produce models that capture the heat transfer at the various interfaces.

Graphite Dimensional Changes in irradiation and temperature fields. These changes lead to variable flow patterns in the reflector blocks (and core blocks in a prismatic HTR) that result in uncertain bypass flows and therefore uncertainty in core fuel temperatures. The usual treatment is to assume that some percent of flow bypasses the core and hope it is conservative. The advanced approach will be linked to the concept of using a coupled thermo-mechanical-CFD simulation to provide increased insight into this issue.

Larg- scale parallel commercial CFD simulation. The NGNP project will require large-scale simulation of turbulent flow in a complex 3-D geometry with important thermal mixing. Understanding something about mesh generation, mesh quality, and mesh convergence (verification) will be very important to make a credible case for validation. Being able to perform large scale parallel simulations on High Performance Computing system environments will also be important.

q. Boussinesq approximation: the idea of the turbulent or eddy viscosity being used as the basis for a simple time-averaged turbulence closure. 


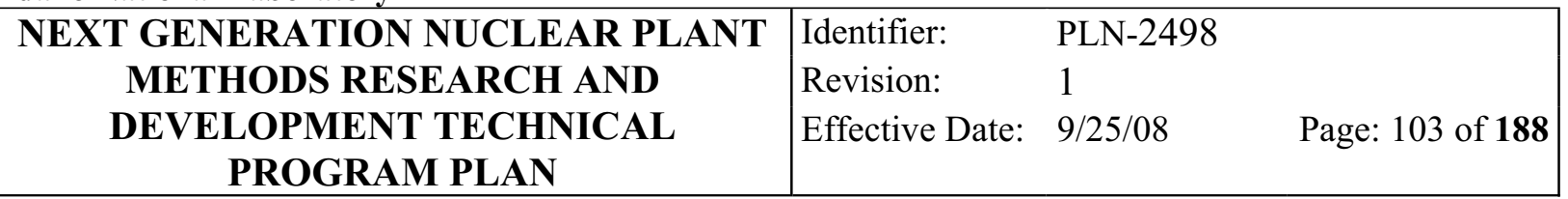

The key technical issues identified by the PIRT (see Appendix A), as summarized in Table 2, are the basis for defining the code development, validation, and analysis $R \& D$ program. The following sections are based on six key technical areas:

- Core heat transfer

- Lower and upper plenum coolant flow

- $\mathrm{RCCS}$

- Air ingress

- Integral system behavior

- Fission product transport.

\subsubsection{Core Heat Transfer Model Validation, Development, and Analyses}

Both systems analysis and CFD software will be used to analyze core heat transfer. CFD is presently being used to calculate the maximum coolant jet temperature into the lower plenum for a baseline prismatic reactor. Systems analysis is used to analyze the distribution of the helium in the core and also the influence of the bypass on the overall core pressure distribution.

\subsubsection{Convective Heat Transfer during Normal Operation}

During normal operation, a nonuniform power and flow distribution in the core will give rise to hot channels. Helium, as a working fluid, experiences an increase in viscosity that is proportional to the absolute temperature raised to the 0.7 power. At the same time, the heat transfer coefficient decreases. Hence, helium flow through hot channels in the core region of both prismatic and pebble-bed cores will tend to decrease, while the flow in cooler channels will tend to increase because of this effect. Analysis is required to determine the maximum fuel temperature during normal operation, the maximum variation in channel outlet temperatures exiting the core into the lower plenum, and the effect of redistributing the flow between the channels. Analysis is also required to study the behavior of variations in the specified design such as inlet orificing.

CFD analyses, started in FY 2005, are aimed at investigating the influence of the turbulence in the hottest cooling channel on the heat transfer and, hence, the subsequent exit temperature. The maximum exit temperature is instrumental in determining the potential for local hot spots in the lower plenum and various structural components as the gas moves to the intermediate heat exchanger or turbine inlets. Also, the potential for hot-streaking at the intermediate heat exchanger or turbine inlets is linked to the peaktemperature coolant jets that enter the lower plenum. Although some analyses were done in FY 2004 and 2005, additional analyses will be performed in subsequent years to evaluate additional geometry configurations, geometry/manufacturing dimensional uncertainties, and conditions once the CFD software are better validated. Experimental data to be used for validation include heated experiments (Section 6.1.1) to evaluate the channel behavior under conditions where asymmetrical heat transfer loads are imposed on cooling channels (FY 2006 and FY 2007).

Systems analysis calculations to consider the redistribution of coolant channel flow as a function of the local peaking factors (hot channel flow) and variations in bypass flow as a function of core life will be performed for the various VHTR reactor design stages. These calculations are necessary to establish the temperature characteristics of the system and the environmental losses during operational conditions. The 


\begin{tabular}{|c|lll|}
\hline NEXT GENERATION NUCLEAR PLANT & Identifier: & PLN-2498 \\
METHODS RESEARCH AND & Revision: & 1 & \\
DEVELOPMENT TECHNICAL & Effective Date: & $9 / 25 / 08$ & Page: 104 of 188 \\
PROGRAM PLAN & & & \\
\hline
\end{tabular}

core heat transfer will be considered in conjunction with the system calculations described in Section 6.2.6, including the effects of fuel depletion on power distribution and control rod insertion.

The friction factor model in RELAP5-3D applies for isothermal conditions. The code has a model to represent the effects of heated transfer on the friction factor based on the ratio of the wall and bulk fluid viscosities. The model applies for liquids and gases. According to Kayes and Crawford (1980), the wallto-bulk temperature ratio is generally used in the correction of the friction factor for nonisothermal conditions in a gas. Furthermore, the effect of heating on the friction factor is opposite for liquids and gases; heating causes the friction factor to decrease in a liquid, but to increase in a gas. The current liquidbased model thus predicts the wrong trends for heating in a gas. Kays and Crawford also show that the effect of heat transfer on the friction factor is much larger for laminar flow than for turbulent flow. Both the laminar and turbulent regimes will be important for analysis of the NGNP. The models for representing the effects of heat transfer on friction factor should be improved for analysis of the NGNP.

\subsubsection{Convective Heat Transfer during PCC}

Core flow behavior following a PCC event will be accompanied by internal vessel recirculation with flow upward through the core in hotter coolant channels and downward through the core in cooler coolant channels and bypass paths. Natural convective heat transfer within the core will include several regimes not currently available in RELAP5-3D. Accurately calculating core fuel temperatures will depend on this convective heat transfer as well as the conduction and thermal radiation that will transfer heat out of the vessel to the RCCS.

CFD validation calculations will be performed based on available mixed convection data (McEligot, Magee, and Leppert 1965; Perkins and McEligot 1975; Reynolds 1968; Shumway 1969; Vilemas and Poskas 1999; Bae et al. 2004) to demonstrate the capability of the CFD tools to adequately calculate the appropriate fluid and heat transfer behavior. Following validation, a CFD code may be used during FY 2007 to evaluate the core heat transfer in conjunction with the flow distributions in the lower and upper plenums to calculate the potential for localized hot spots in the vessel upper head and control rod apparatus (see Section 6.2.3).

Also, the correlations developed on the basis of available mixed convection data (McEligot, Magee, and Leppert 1965; Perkins and McEligot 1975; Reynolds 1968; Shumway 1969; Vilemas and Poskas 1999; Bae et al. 2004) will be evaluated for applicability and installed in the systems analysis software. The validation calculations will demonstrate the capability of RELAP5-3D to adequately represent these mixed convection regimes (FY 2005). Following confirmation of the code's capability to perform such calculations RELAP5-3D will be coupled with a CFD code to enable the modeling of the core flow into the upper plenum and then down through the cooler channels to the lower plenum.

Axial and radial conduction during PCC and DCC. Decay heat removal following a PCC or DCC event will be accompanied in part by axial as well as radial heat transfer within the core. The decay heat removal rate is a key ingredient in determining the peak fuel temperature and the peak structural temperatures in the reactor vessel. Hence, confirmation of the software's capability to calculate this behavior is crucial. This R\&D consists of (a) revision of the heat structure modeling capability of RELAP5, (b) validation calculations based on the expected behavior of a prismatic reactor during a conduction cooldown event, (c) validation calculations based on the AVR experimental data, and (d) validation calculation based on the Sana I experimental data. 


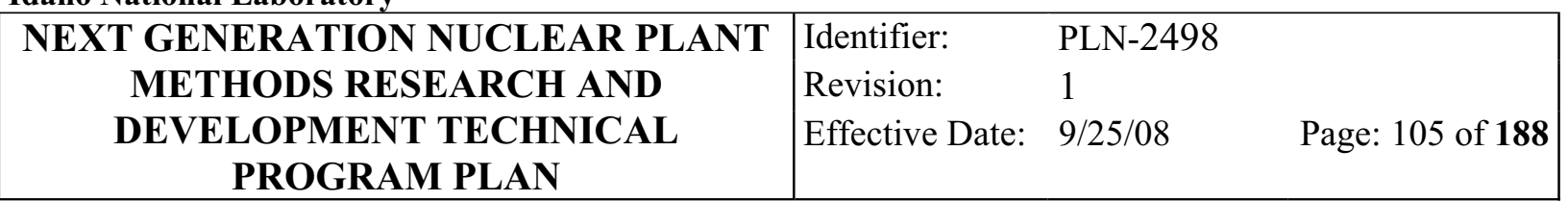

\subsubsection{Revision of Existing RELAP5-3D Heat Structures}

RELAP5-3D only calculates heat transfer between heat structures in the radial direction (via contact conductance as well as thermal radiation). In either the prismatic or pebble-bed core design, the core structure will be modeled using a series of RELAP5-3D heat structures. Therefore, this task will implement the same heat transfer mechanisms in the axial direction (FY 2006).

This modeling approach for prismatic block core designs (see example in Figure 36) assumes each graphite block has a uniform temperature, which should prove adequate for modeling core behavior during PCC and DCC events. However, a more detailed model is needed to capture the elements of the core geometry and predict the temperature response at the smallest scale, called the "primitive." Figure 37 shows the primitive generally used for calculating heat transfer in prismatic block reactors. The primitive forms the link between a RELAP5-3D model that represents each block in the prismatic reactor and studies that will be done using a code such as Fluent or Abaqus to subdivide each block into a large number of mesh cells to obtain a detailed temperature distribution within the blocks (FY 2006).

The plan is to use data from the HTTR facility to perform the needed validation calculations for the prismatic block type VHTR. The HTTR, described in Section 6.1.5.3, is an operating prismatic test reactor. Both PCC and DCC scenarios are planned for the HTTR in the future. Data from these experiments (if the facility is appropriately instrumented) will enable a thorough validation of the systems analysis code's calculational capability (FY 2007).

Data from the AVR German PBR will be used to validate core heat transfer modeling in pebble-bed VHTRs. The reactor operated from 1967 to 1988 and was the only nuclear power plant that was intentionally subjected to a lossof-coolant event without emergency cooling (Krüger et al. 1991). The data includes core power and temperatures measured at various locations in the reactor vessel including the reflectors, reactor shroud, inner vessel, and within instrumented pebbles. The data are considered valuable for qualitative validation of pebble-bed modeling. The validation effort and will provide conclusions on the capability of a coupled RELAP5-3D/Fluent model to properly predict pebble-bed system behavior (FY 2008).

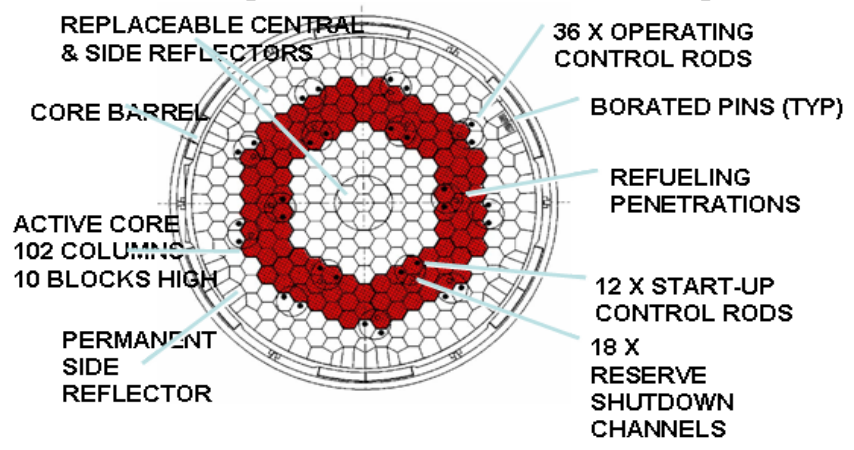

Figure 36. Prismatic core layout.

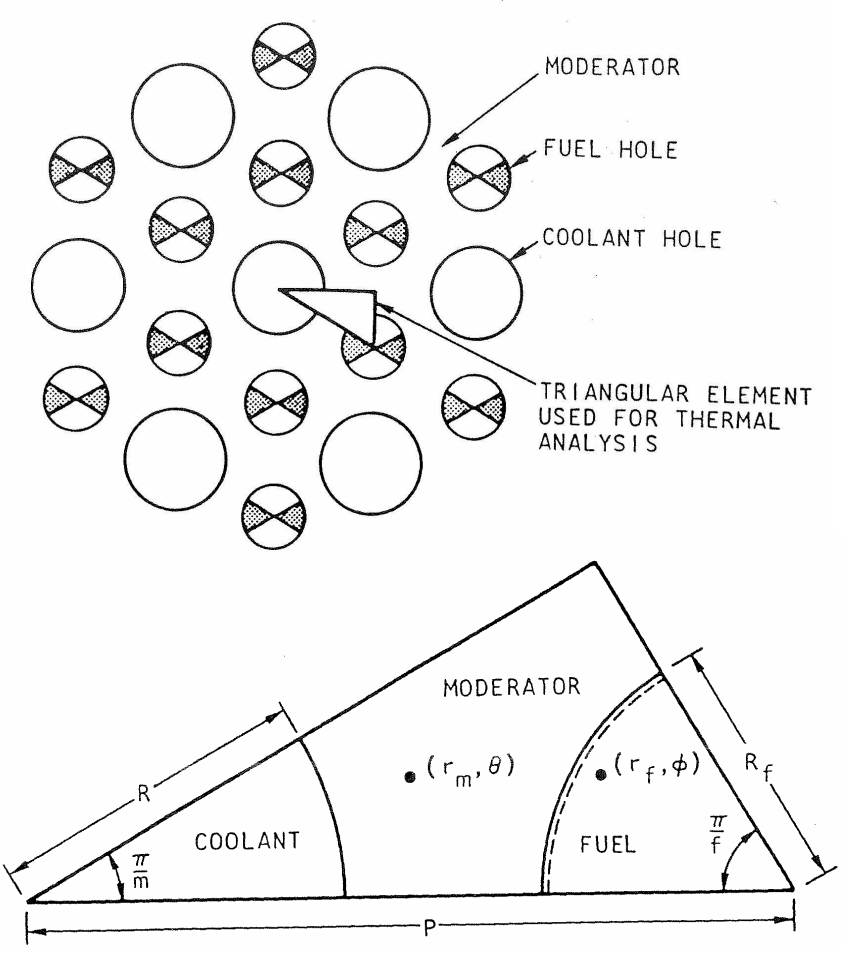

Figure 37. Prismatic block reactor core primitive. 


\begin{tabular}{|c|lll|}
\hline NEXT GENERATION NUCLEAR PLANT & Identifier: & PLN-2498 \\
METHODS RESEARCH AND & Revision: & 1 & \\
DEVELOPMENT TECHNICAL & Effective Date: & $9 / 25 / 08$ & Page: 106 of 188 \\
PROGRAM PLAN & & & \\
\hline
\end{tabular}

Data from the German Sana-I facility shown in Figure 38 (IAEA 2000) will also be used in validating pebble-bed modeling. The test rig was designed to study the heat transfer mechanisms in a pebble-bed core filled with 9,500 graphite pebbles (diameter $=6 \mathrm{~cm}$ ) and to provide the basis for validating the models required to determine whether sufficient energy can be transferred to the environment to prevent the fuel from becoming damaged following failure of all heat sinks with a simultaneous depressurization. These data will be used to validate RELAP5-3D through simulations of the experiment (FY 2008).

\subsubsection{Core Bypass}

In FY 2007, a scoping study will be initiated to assess the influence of the bypass and the factors that determine the sensitivity of the bypass flow. The bypass flow passes through the reflector regions in both pebble-bed and block reactors and, in a block-type reactor, between the blocks. Because the quantity of bypass flow is a direct function of the bypass area, which in turn is a function of the temperature distribution, fluence, and graphite properties, the influence of the bypass on the core temperature distribution may be significant and vice versa. The influence of bypass flow in the block type reactor may be assessed in part by performing a series of parametric calculations that differ in the geometric boundary conditions as defined by the various factors that influence the bypass flow passages such as manufacturing tolerances, misalignments, and geometric distortions. Parametric calculations for the pebble-bed will be performed using varying axial packing fraction and temperature profiles. During the predesign selection phase, flow modeling requirements in terms of network tools vs. CFD for the inter-column gap leakage for the prismatic reactor, and in the case of the PBR, the porous media effective conductivity dependence on the local void fraction, will be included in the study.

New research conducted in U.S. universities will be particularly helpful in this area. For example, university research on the materials and structural modeling and associated analysis/validation work for the structural stability of graphite, in the case of the prismatic reactor type, would be very useful. In the case of the PBR, university research on the modeling and analysis/validation of local flow between the pebbles would be very useful. It is envisioned that interactions between the NGNP Project and the university research centers will be guided on the basis of results of the scoping studies. This would also be true for the planning of the largescaled NSTF bypass flow experiments. The scoping studies should include a preliminary scaling analysis to determine the feasibility of the modifications required for the large-scale NSTF bypass flow

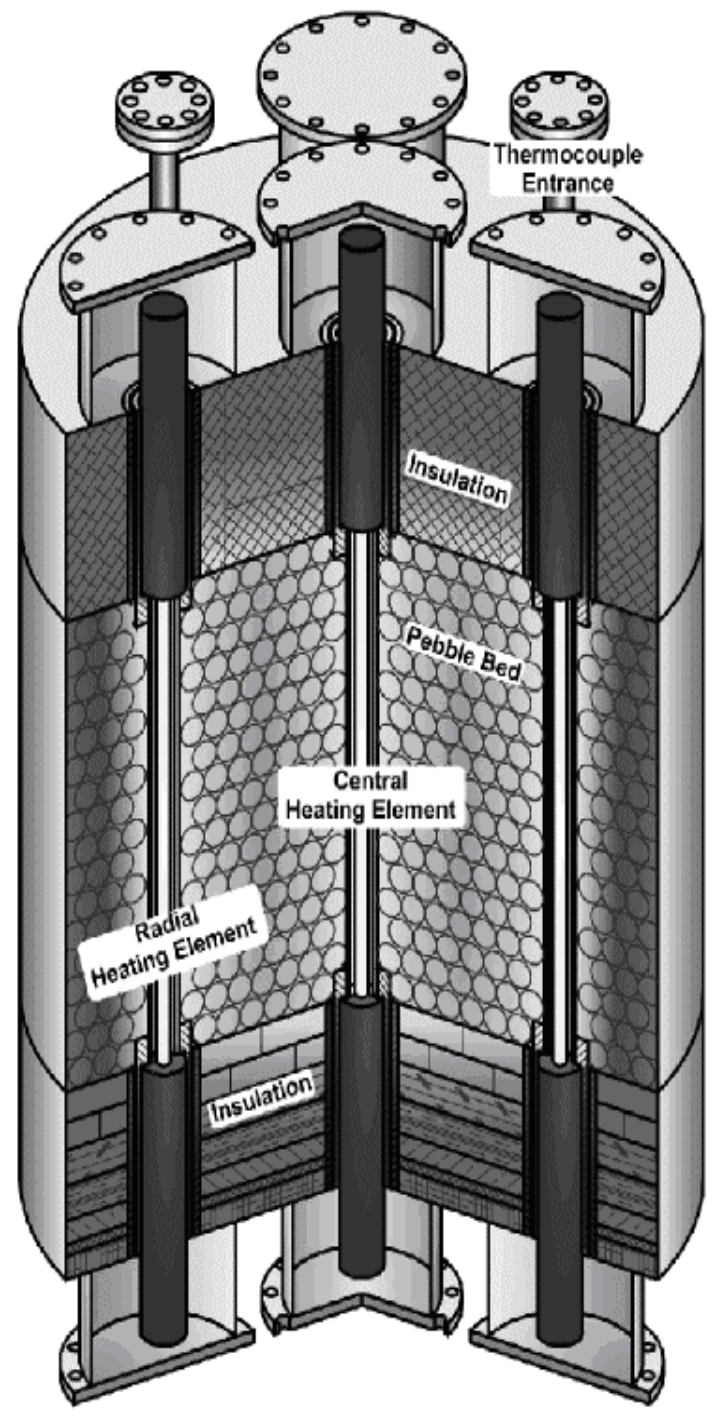

Figure 38. SANA-I facility. 


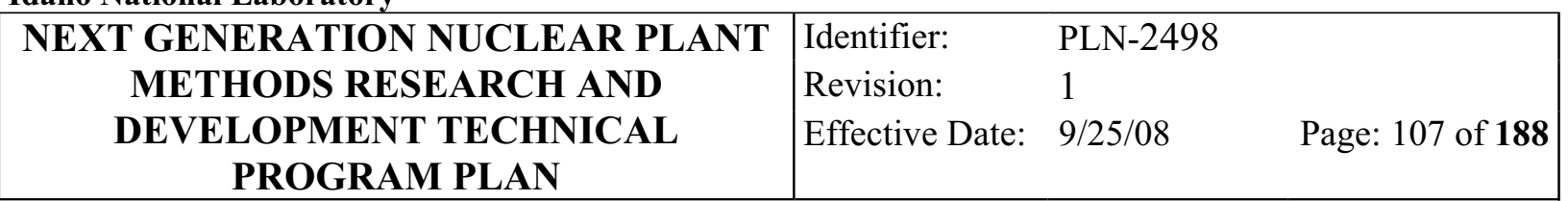

experiments. Once feasibility has been determined, analysis will be performed in FY 2008 in support of detailed experiment planning. This will be factored into the selection of experiment scale, size of blocks and pebbles materials and the requirements on flow and temperature profiles. A combination of CFD and university models will be used as the pretest and blind test tools. Post initiation of the NSTF bypass flow test, validation of these tools will be carried out against the large-scale test data in FY 2009-2011.

\subsubsection{Upper and Lower Plenum Flow Mixing Validations, Development, and Analyses}

\subsubsection{Lower Plenum}

Introduction of the hot jets from the core into the lower plenum creates the potential for the hottest jet streams to impinge on the lower plenum structural surfaces in regions of low cross-flow. It also creates the potential to move into the hot duct and then to the intermediate heat exchanger or turbine inlets via flow streams passing through low mixing regions. Given that the hottest jets will have exit temperatures well in excess of the average exit temperature, a rigorous, accurate evaluation of the peak temperature jet behavior and the interactions of these jets with adjacent jets, and especially their interactions with the lower plenum structural materials, are crucial. Figure 39 shows a preliminary calculation of the mixing behavior in the lower plenum of a representative advanced gas-cooled reactor design (produced in the collaborative effort between Fluent, General Atomics, and INL). To calculate mixing in the lower plenum, the validation effort will focus on CFD software.

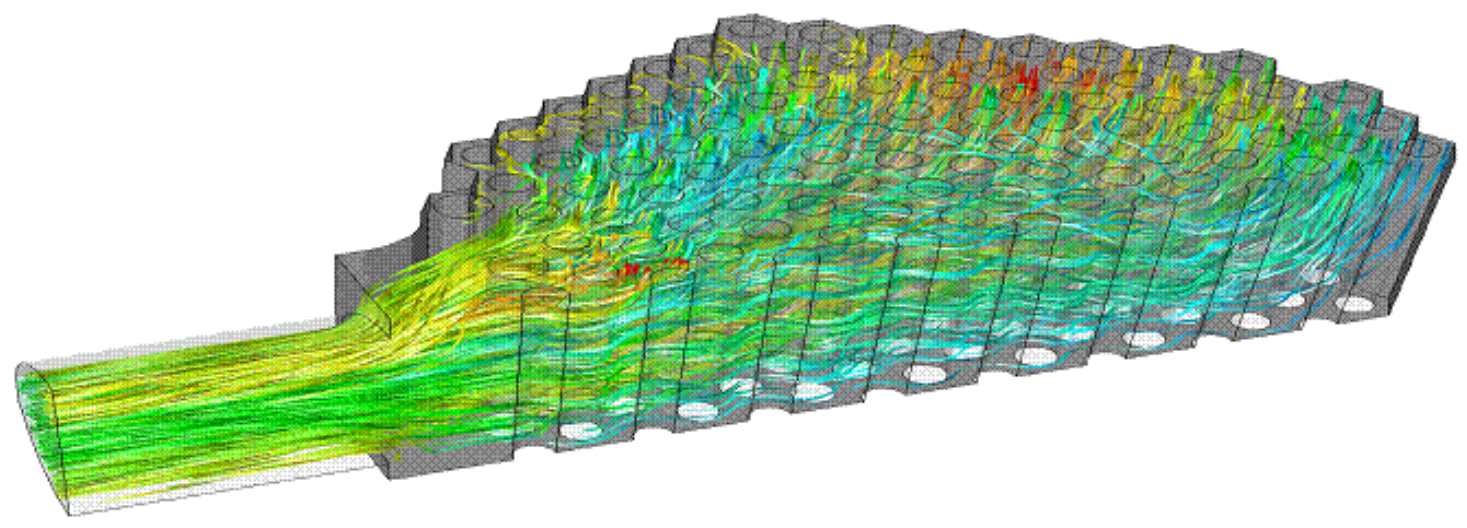

Figure 39. Preliminary calculation of mixing in prismatic reactor lower plenum. Courtesy of Fluent, Inc and General Atomics Corp.

The following experimental validation sets will be used to validate the CFD model that will be used to calculate mixing in the lower plenum:

- Benchmark Calculations of Mixing Behavior in the Lower Plenum. The turbulent intensity and turbulence as a function of location in the VHTR design lower plenum will be calculated and evaluated. The benchmark calculation will be used as a frame-of-reference for the scaled lower plenum experiments and jet/cross-flow data (e.g., MIR), jet, and cross-flow interactions data (FY 2005). In addition to serving as an experiment design tool, this calculation will be a baseline that is used as a basis of comparison or starting point for the validation. As the CFD code is validated and a 


\begin{tabular}{|c|lll|}
\hline NEXT GENERATION NUCLEAR PLANT & Identifier: & PLN-2498 \\
METHODS RESEARCH AND & Revision: & 1 & \\
DEVELOPMENT TECHNICAL & Effective Date: & $9 / 25 / 08$ & Page: 108 of 188 \\
PROGRAM PLAN & & & \\
\hline
\end{tabular}

more detailed understanding of the mixing behavior is obtained, the baseline calculation will be updated and studied in more detail.

- Matched-Index-of-Refraction (MIR) Experiments. CFD models of the MIR experiments described in Section 6.1.2 will be constructed and validation calculations will be performed. The emphasis will be on evaluating the turbulence models and relating the scaled experiments to the fluid behavior postulated to occur in the VHTR design. (FY 2005 to FY 2007)

- Heated Experiments. The evaluation and validation calculations will be performed by constructing a CFD mod Since the MIR experiments are isothermal, they will not reveal the buoyancy contribution because of temperature variations in both the inlet jets and cross-flow. This effect will will be evaluated through a series of heated experiments. (see Figure 39). The turbulence intensity and turbulence behavior as a function of location will be compared with the data (FY 2005 to FY 2008).

- Benchmark validation studies based on jet and cross-flow data (Schultz, Ball, and King 2004). Data describing the general jet and cross-flow phenomena, including the interactions between jets and cross-flow, will be used to perform separate effects validation studies of the Fluent code (FY 2005).

\subsubsection{Upper Plenum Flow}

Following a PCC event internal vessel recirculation flow will occur in which helium coolant will flow upward through the core in the hotter coolant channels and downward through the core in the cooler coolant channels and bypass paths. These flows will mix in the lower and upper plenums. The recirculation will cause heating of the upper plenum structure where local temperatures may approach the limiting values for the structural materials or the control rod apparatus. Validation calculations are required to ensure VHTR reactor analyses of the vendor's design give reasonable results. The coupled RELAP5-3D/Fluent model will be used to analyze the PCC event. Validation of the modeling approach will be accomplished by using the data from the planned upper plenum plume experiments (Section 6.1.2) (FY 2009).

\subsubsection{Reactor Cavity Cooling System Validations, Development, and Analyses}

The RCCS is the primary system for transferring the core residual and decay heat to the environment following a PCC or DCC event. Consequently, the RCCS plays an important role in determining core temperature distribution, peak fuel temperatures, and peak structural member temperatures for a given design and power rating. Also, since the RCCS operates in the "null" mode during rated operational conditions with a noticeable fraction of the generated power transferred to the environment, the RCCS has some influence on the plant efficiency and operational conditions (core temperature distributions, etc.). The software tools must therefore be capable of accurately predicting the system behavior under all conditions.

The RCCS design will depend in large measure on whether a low-pressure filtered and vented system confinement is allowable or a high-pressure containment is required by the regulators. If a confinement is allowable, the RCCS may likely be air-cooled. If a containment is required, the RCCS will likely be water-cooled. In either case the largest fraction of the energy transferred from the reactor vessel to the reactor cavity walls occurs through radiation heat transfer. Heat transfer from the walls to the environment may be either through a natural circulation-driven air-cooled duct system or through a watercooled sleeve. A smaller fraction of the energy transferred to the reactor cavity walls occurs through convective heat transfer via natural circulation of the gases enclosed in the reactor cavity. A validated model is required to analyze the heat transfer via radiation and convection from the reactor vessel to the environment. A conceptual plan view of such a system is shown in Figure 40. 


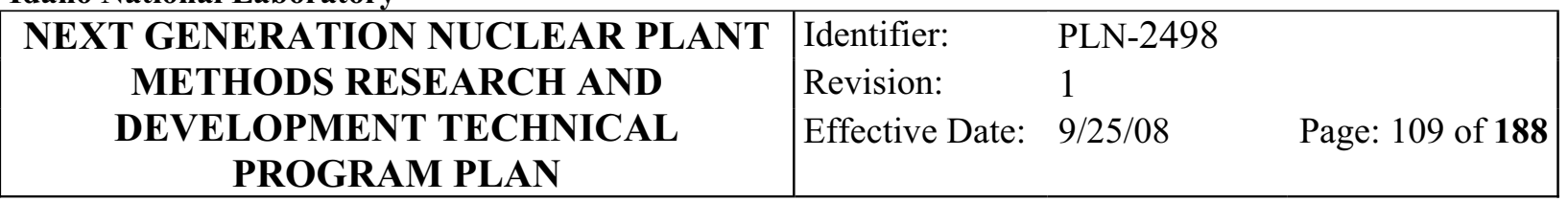

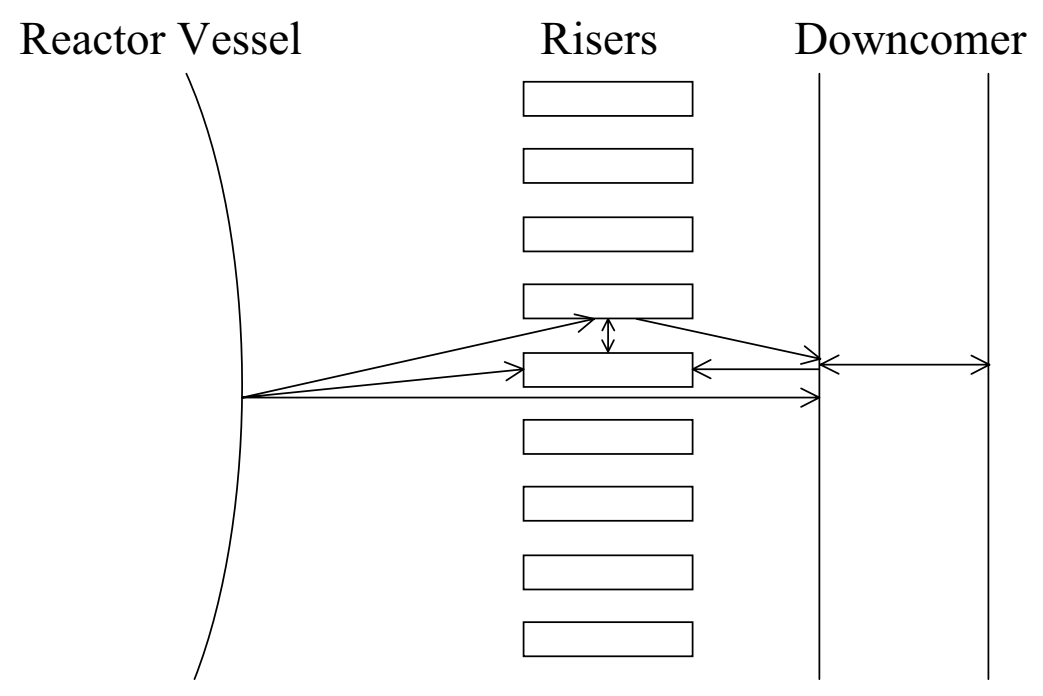

Figure 40. Concept for an RCCS design.

The following experimental validations and analyses are planned to validate a model's capability to calculate the RCCS behavior.

\subsubsection{ANL Air-Cooled RCCS (see Section 6.3)}

The validation/analysis effort will consist of developing the experimental matrix and performing facility and pre-experimental design calculations, blind calculations, and post-test calculational analysis. The experiment matrix will be developed based on review of vendor data, if available, and performance of plant system calculations for the range of selected RCCS candidate designs. The matrix will cover the operational conditions, depressurized conduction cooldown, and pressurized conduction cooldown.

For the selected candidate RCCS systems, the RELAP5/ATHENA system models will be constructed and a range of accident scenarios will be studied to obtain the range of boundary conditions for overall global parameters. Operational conditions and both depressurized and pressurized conduction cooldown will be enveloped. A set of either STAR CD or FLUENT models will be defined for the selected candidate RCCS systems, and the corresponding CFD calculations will be performed for a selected set of driving boundary conditions within the range determined by the RELAP5/ATHENA results. These results and the results of the scalability study will be used in the selection of the matrix and the derivation of experiment criteria.

Empty cavity, single tube and multiple tube experiments will be considered in the matrix planning for baselines. Since the facility operates in one of two thermal modes, (a) constant (uniform) wall temperature or (b) constant (uniform) heat flux, both will be used. The system also accommodates stepwise variation of either mode singly, or both in combination, so this feature will be used as well in the definition of the experiments. A selection will be made based on the relevant decay heat levels, vessel wall temperatures, and power profiles based on the RELAP5/ATHENA system results. Because of the large thermal time constant associated with the experimental facility, thermal transient analyses will be performed with models of the experiments to define experiment start-up procedures as part of the 


\begin{tabular}{|c|lll|}
\hline NEXT GENERATION NUCLEAR PLANT & Identifier: & PLN-2498 \\
METHODS RESEARCH AND & Revision: & 1 & \\
DEVELOPMENT TECHNICAL & Effective Date: & $9 / 25 / 08$ & Page: 110 of 188 \\
PROGRAM PLAN & & & \\
\hline
\end{tabular}

experiment planning. Data analysis techniques, filtering, and experiment diagnostics will be applied to select the appropriate data for archiving in the database. A database will be developed with automatic queries to aid in the use of the information in the code/model validation work. During the predesign selection phase, NSTF RCCS transient analyses will be performed with STAR-CD and RELAP-5 to verify the FY 2006 NSFT scaling recommendations for a reference water-cooled RCCS design and to support the reconfiguration of the NSTF facility to also serve as a simulator of a water-cooled RCCS.

Using part of the experiment database information, 1-D models/correlations will be developed that can be implemented in the RELAP5/ATHENA system codes. The RELAP5/ATHENA models will be used to obtain measures to compare against other parts of the database. The results of the scalability study will be used to select the appropriate groups of nondimensional variables. Based on the RELAP5/ATHENA system calculations and STAR CD or FLUENT calculation of candidate RCCS systems used in the selection of the experiment matrix, the plant conditions will be classified into separate phenomenological ranges. STAR CD or FLUENT CFD calculations will be performed for experiment planning and the results acquired from the performance of the experiments will be used to determine the optimum form for the correlation fit. The data analyses required to produce the optimum correlation fits with the selected scaling variables for each category of range of conditions will be performed. ANL will work with the RELAP5/ATHENA code developers to implement any new models/correlations in the code. Appropriate validation data from the part of the database that was not used in the model/correlation development will be selected and the models/correlations will be tested against those experiments.

For CFD code validation, contributions will be made to the reviews of the experiments, experiment plans and procedures, and procedures and comparisons against the experiments in the matrix will be performed. A STAR CD or FLUENT model of the experiment configuration will be constructed. Parametric studies will be performed to define the meshing. For experiments in the matrix, CFD calculations with the given boundary conditions will be performed. These results and the results of the scalability study in the design and planning of the experiment will be used to aid in the location and selection of instrumentation. The geometry of the experiments, gap size, tube size and spacing will be set. The results will be analyzed to confirm that experiment goals and criteria can be achieved. Because of the large thermal time constant associated with the experimental facility, thermal transient analyses will be performed with models of the experiments to define experiment start-up procedures as part of the experiment planning. Data analysis techniques, filtering, and experiment diagnostics will be applied to select the appropriate data for archiving in the database. A database will be developed with automatic queries to aid in the use of the information in the code/model validation work. Once the experiments are performed, the Star-CD or Fluent CFD codes will be validated against the experimental database for the prediction of RCCS performance under operational conditions, depressurized conduction cooldown, and pressurized conduction cooldown. Appropriate meshing strategies, structures and length scales for RCCS modeling will be studied for each condition. Appropriate turbulence modeling strategies (RANS, LES, DNS) and numerical schemes for each condition will be identified and used.

\subsubsection{Seoul National University Water-Cooled RCCS (see Figure 32)}

A similar validation effort, to that described above for the ANL air-cooled RCCS experiments, will be performed. However, because some two-phase behavior will likely occur in the water-filled thermal sleeve, RELAP5-3D or the CFD code NPHASE will be used (FY 2009). 


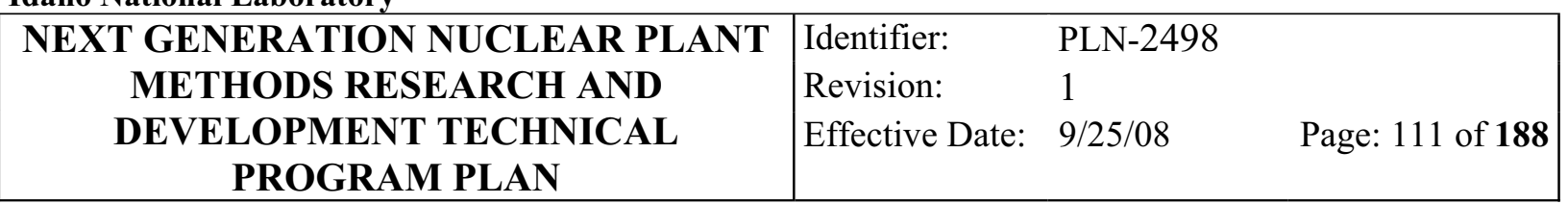

\subsubsection{HTTR RCCS Separate-Effects}

Validation will be performed using existing experimental data from the HTTR mockup experiments (IAEA 2000). Data were obtained in a series of six tests performed to simulate the heat transfer to the RCCS cooling panels.

For these experiments, cooling panels were placed inside a pressure vessel (Figure 41) and the gas in the pressure vessel was varied to change the natural convection characteristics. Vacuum (heat transfer from the heaters to the cooling panels by radiation alone), nitrogen; and helium were used. The cooling medium in the cooling panels was water for four experiments and air for three experiments. The power level was also varied. These data will be used for validation of a coupled RELAP5-3D/Fluent model to correctly calculate and predict RCCS behavior. A report will be issued documenting the validation effort and will provide conclusions on the capability of the codes to properly predict RCCS behavior (FY 2008).

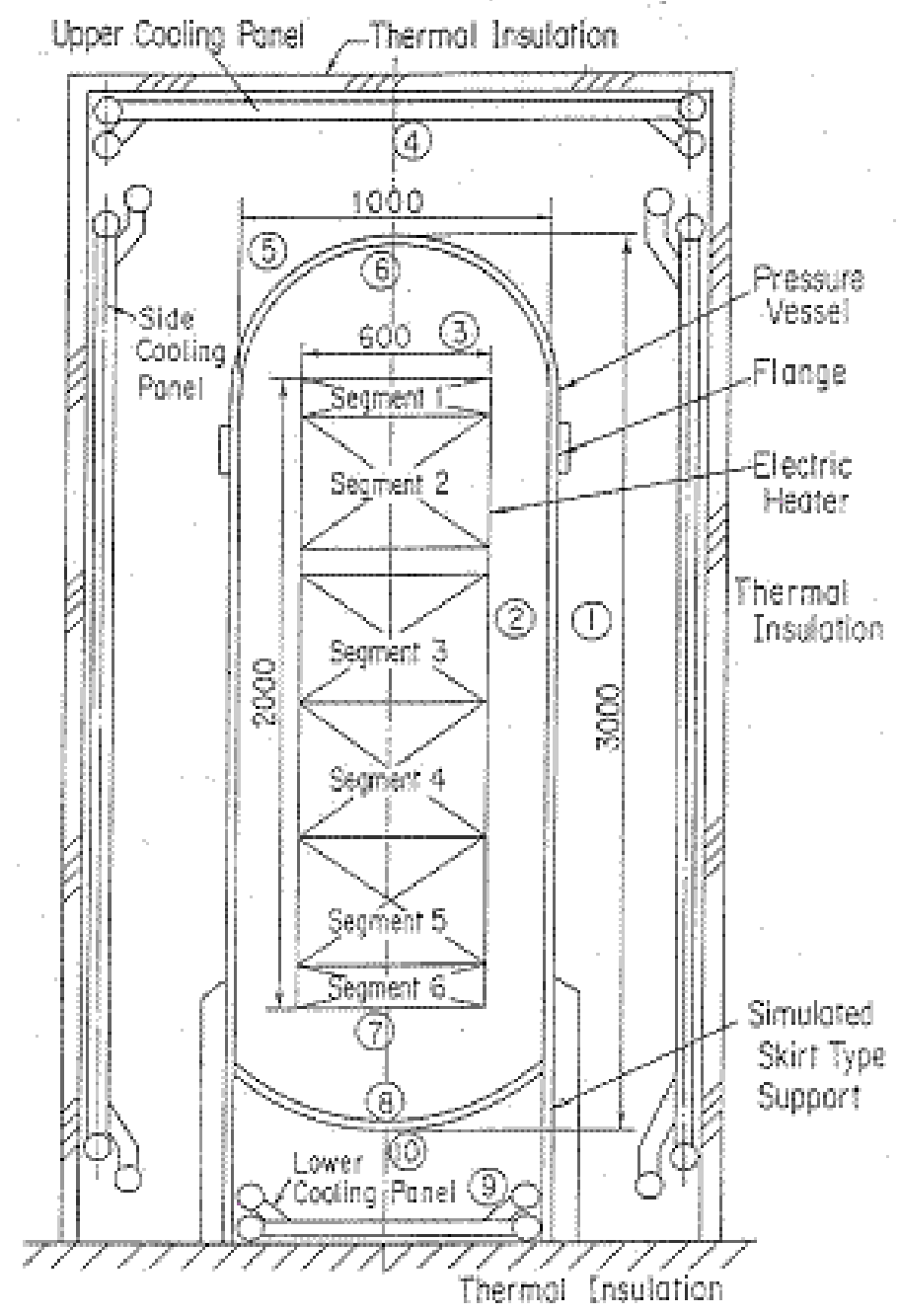

Figure 41. HTTR Mockup Facility 


\begin{tabular}{|c|lll|}
\hline NEXT GENERATION NUCLEAR PLANT & Identifier: & PLN-2498 \\
METHODS RESEARCH AND & Revision: & 1 & \\
DEVELOPMENT TECHNICAL & Effective Date: & $9 / 25 / 08$ & Page: 112 of 188 \\
PROGRAM PLAN & & & \\
\hline
\end{tabular}

\subsubsection{Air-Ingress Validations, Development, and Analyses}

Following a LOCA, air ingress into the vessel may result in exothermic graphite oxidation, aggravating the core cooldown and resulting in potential core damage. The process driving the exchange of vessel and containment/confinement gases will be molecular diffusion. This diffusion process will involve several gases, including helium, nitrogen, oxygen, carbon monoxide, and carbon dioxide.

Air ingress scenarios will be calculated using RELAP5-3D alone and using Fluent coupled to RELAP5-3D. Because the air that diffuses into the reactor lower plenum will probably do so nonuniformly, a CFD tool will be required to produce a 3-D air distribution in the lower plenum using first-principles. Hence it is essential to validate the capability of the CFD software to calculate air diffusing into a plenum occupied by helium. In the event that the air distribution into the lower plenum can be shown to be calculable using a 1-D systems analysis, the use of RELAP5-3D alone may be adequate. Thus it is also essential to validate the capability of RELAP5-3D to perform the air ingress calculation. If a CFD analysis of air diffusion into the lower plenum is required, the CFD software will be used coupled to RELAP5-3D, which will have the capability to calculate exothermic graphite oxidation.

RELAP5-3D currently has a two-species (helium and nitrogen) diffusion model. The model will be extended to a multispecies model. An additional model will be implemented for graphite oxidation, which will represent both the accompanying heat generation and mass consumption of oxygen and generation of $\mathrm{CO}$ and $\mathrm{CO}_{2}$. A report documenting the verification of these models will be issued (FY 2006). Planned validation studies for both the CFD software and RELAP5-3D include:

- Takeda and Hishida Experiments. The experiments performed by Takeda and Hishida centered on a reverse U-shaped tube configuration and a simple model of the HTTR. The studies will focus on the flow behavior of multicomponent gas mixtures because of molecular diffusion and the natural circulation of the multicomponent gas mixture (FY 2007).

- NACOK Experiment. The NACOK experiments (Schaaf et al. 1997) were designed to model a representative section of a VHTR core undergoing the effects of air ingression following a LOCA. Therefore, the data from these experiments are suitable for validating diffusion modeling capability. A RELAP5-3D and Fluent model will be constructed and validation calculations will be performed. A report documenting the validation effort will be issued that provides conclusions on the capability of the codes to properly predict system behavior (FY 2008).

- Helium/Air Heat Transfer Experiments. Data from experiments planned for FY 2006 to 2007 will provide the basis for validation of heat transfer and pressure drop in the core for mixtures of air and helium (FY 2008).

The DCC event creates the potential for the release of fission products into the confinement or containment. The release of fission products from the fuel (including radioactive dust in the case of the PBR) transport within the coolant system and confinement, and the deposition of these products must be calculable. To provide these capabilities in an overall systems code approach, the inherent models in PARFUME (fission product release) will be augmented by the capabilities of a code such as VICTORIA (fission product transport and deposition). These codes will be linked to RELAP5-3D in FY 2010 using the existing PVMEXEC protocol. A report will be issued demonstrating the verification of the coupled models (FY 2010). 


\begin{tabular}{|c|lll|}
\hline NEXT GENERATION NUCLEAR PLANT & Identifier: & PLN-2498 & \\
METHODS RESEARCH AND & Revision: & 1 & \\
DEVELOPMENT TECHNICAL & Effective Date: & $9 / 25 / 08$ & Page: 113 of 188 \\
PROGRAM PLAN & & & \\
\hline
\end{tabular}

\subsubsection{Integral System Behavior Validations, Development, and Analyses}

The ultimate objective of the VHTR Program software validation and qualification effort is to demonstrate the capability of the required software to produce calculations that describe the VHTR integral system behavior with acceptable accuracy for operational conditions and off-normal or accident conditions. The focus of these essential calculations is usually the location and magnitude of the peak fuel temperatures and peak structural temperatures, although other variables will be identified that are important (peak structural loads, peak power under adverse conditions and operational conditions, conditions that lead to adverse operating conditions for the intermediate heat exchanger or the turbine, potentially damaging oscillatory conditions, etc.). Because the VHTR reactor has many components, the net system behavior is described properly when all of the system component interactions are accurately calculated. The VHTR system model will require both development and validation. Development tasks consist of:

- Concurrent Fluent Models. A coupled RELAP5-3D/Fluent model will be used to model the reactor vessel for the PCC events, in which Fluent will model the inlet and outlet plenums and RELAP5-3D will model the core region. Fluent is needed for the upper and lower plenum modeling to capture the 3-D flow patterns that will occur in these regions as coolant circulates within the vessel. To accomplish this, the present capability to link RELAP5-3D models to Fluent models using the coupling protocol will be extended to enable two separate Fluent models communicating with the RELAP5-3D model. A report will be issued documenting the dual coupling capability in FY 2007.

- Coupled Neutronics. It may be necessary to model part of the core region (prismatic or pebble-bed) using Fluent and the remainder with RELAP5-3D to enable a direct comparison of coolant channel flow behavior between the codes under the same conditions - a code-to-code validation. This is presently possible except for the exchange of neutronic data between the codes, even though the mechanism to exchange neutronic data has been developed. This task will extend the coupling capability to allow neutronic data to be exchanged between the codes during each time step. A report will be issued demonstrating this capability in FY 2009.

- RELAP5-3D/PEBBED Linkage. PEBBED will be the neutronic module for the PBR. This task will link PEBBED with RELAP5-3D, providing a complete thermal hydraulic and neutronic systems analysis package, assuming a pebble-bed design has been chosen by then. The linkage strategy will be the same as that currently employed with the NESTLE code imbedded in RELAP5-3D. A report will be issued documenting the verification of the linkage in FY 2009.

- Balance-of-Plant Components. Data will be acquired that are representative for turbines, compressors, and reheaters planned for use in the VHTR. Models of these components will be developed to perform the necessary validation. RELAP5-3D already contains the modeling elements required for these components. The design data are needed to build the system-wide VHTR model and should be available by FY 2009. A report documenting the basis for these models will be issued in FY 2009.

- Intermediate Heat Exchanger. The VHTR system model will require a model for the intermediate heat exchanger that couples the coolant system to the hydrogen production system. By FY 2009, sufficient information should be available to enable the incorporation of a mathematical model of the intermediate heat exchanger into RELAP5-3D. A sufficient representation of the hydrogen production system interface will be implemented through the use of RELAP5-3D control blocks. A report will be issued demonstrating the functionality of the intermediate heat exchanger model as compared to design specifications in FY 2009. 


\begin{tabular}{|c|lll|}
\hline NEXT GENERATION NUCLEAR PLANT & Identifier: & PLN-2498 \\
METHODS RESEARCH AND & Revision: & 1 & \\
DEVELOPMENT TECHNICAL & Effective Date: & $9 / 25 / 08$ & Page: 114 of 188 \\
PROGRAM PLAN & & & \\
\hline
\end{tabular}

To ensure the coupled CFD/systems code software can properly calculate integral system behavior, a series of validation calculations will be performed using data from the two operational integral facilities (HTTR in Japan and HTR-10 in China). Finally, validation of the system model will use data from the scaled vessel experiments.

\subsubsection{HTTR and HTR-10 Reactors}

The HTR-10 is a Chinese 10 MW pebble-bed gas-cooled reactor that became operational in 2000 and presents an ideal source of data for validation of pebble-bed system modeling. A spectrum of experiments is planned, including perhaps a LOCA and pressurized conduction cooldown. Portions of these data will be available through the International Atomic Energy Agency and the Institute of Nuclear Energy Technology in Beijing. This task will develop a system model of HTR-10 and perform validation calculations.

The HTTR is a $30 \mathrm{MW}$ prismatic gas cooled reactor in Japan. The potential for HTTR experiments to provide high quality data for code validation is great, but may be limited by available instrumentation and restrictions on the range of transients that may be permitted.

\subsubsection{System Performance Enhancement Validations}

Preliminary computational fluid dynamics models will be used to fine-tune the final experimental facility design, aiding in the placement of instrumentation and optimization of inlet/outlet configurations. An experimental test matrix will be developed to ensure that adequate data is captured to provide validation of the experimental tools at bounding and expected conditions.

Computational simulations of selected experiments will be completed using the fluid structure interaction prediction capabilities within a selected commercial CFD code or, if deemed necessary, the coupled fluid dynamics and solid mechanics capability available within the anticipated release of the StarCCM (Complete Continuum Mechanics) code. Alternatively, a standard commercial CFD code coupled to a standard commercial solid mechanics code through the generic coupling interface provided by the Numerical Nuclear Reactor may be used. Additional cases will be reserved for validation of future developments of these capabilities if such developments are deemed necessary by the initial benchmarking activity. An evaluation of the applicability of the existing fluid-structure interaction modules in STAR-CD to VHTR conditions of steady state operation and LBLOCA depressurization will be carried out during the predesign selection phase. Validation data needs and experiment plans will be identified. The focus will be on the outlet plenum.

\subsubsection{System Behavior Calculations}

VHTR system behavior during normal operation and accidents will be performed using RELAP5-3D and Fluent models of the plant at each stage of the design process. The first complete model of the plant will be built after completion of the preconceptual design. Calculations will focus on peak temperatures during the most challenging scenarios. Reports will be issued at each design stage.

\subsubsection{Fission Product Transport}

The Technical Program Plan for the Advanced Gas Reactor Fuel Development and Qualification Program (Petti, Hobbins, Kendall, and Saurwein 2005) discusses the need for experimental facilities for fission product and source term experiments in Section 3.5.1. Under R\&D Task 3.5.11, specific experiments are outlined for fission product transport in the vented low-pressure containment (VLPC). 


\begin{tabular}{|c|lll|}
\hline NEXT GENERATION NUCLEAR PLANT & Identifier: & PLN-2498 \\
METHODS RESEARCH AND & Revision: & 1 & \\
DEVELOPMENT TECHNICAL & Effective Date: & $9 / 25 / 08$ & Page: 115 of 188 \\
PROGRAM PLAN & & & \\
\hline
\end{tabular}

The compartment and spaces in the reactor silo building are connected together to form a long and torturous vent path. During events involving primary coolant leakage into the silo and onto the building, natural processes will act to reduce the level of entrained radio-nuclides as the gas stream transits. Mechanistic radionuclide retention in the VLPC is considered when showing compliance with the Protection Action Guide (PAG) dose limits at the EAB with source terms for core conduction cool down accidents. Data are needed to develop and validate the methods describing the behavior of condensable radionuclides in the building under wet and dry conditions for these accidents. The reactor silo with the cavity and the RCCS piping forms a compartment with internals in this long vent path for the gas transit. Once the NSTF is configured for the RCCS experiment of Section 6.1.3, the structures and geometry for the condensation of the fission products will be available for an integral large-scale experimental simulation of fission product transport in this cavity and silo. A preliminary scoping evaluation will be the starting point to assess the feasibility of using the NSTF to perform multifunction integral experiments. The focus will be simulant fission product transport experiments.

\subsection{Summary: Focus and Direction}

The timelines for the thermal-fluid and structural thermal analysis R\&D and analyses are given in Figure 42. The R\&D is divided into two phases:

- Phase 1 focuses on codifying the methodologies, practices, and procedures for software validations. Phase 1 R\&D is designed to be equally applicable to either the pebble-bed or prismatic VHTR design. Consequently, Phase 1 R\&D includes experiments that are of a more fundamental nature and also analysis efforts designed to qualify software such as CFD, which has never been validated and used for nuclear system analysis purposes to any great extent in the past. Phase $1 \mathrm{R} \& \mathrm{D}$ will continue until a VHTR design is selected. Phase 2 will follow design selection.

- Phase 2 focuses on executing the methodologies, practices, and procedures to validate the thermalfluids software and to perform the required analyses of the NGNP for final design studies, licensing, and plant operational studies.

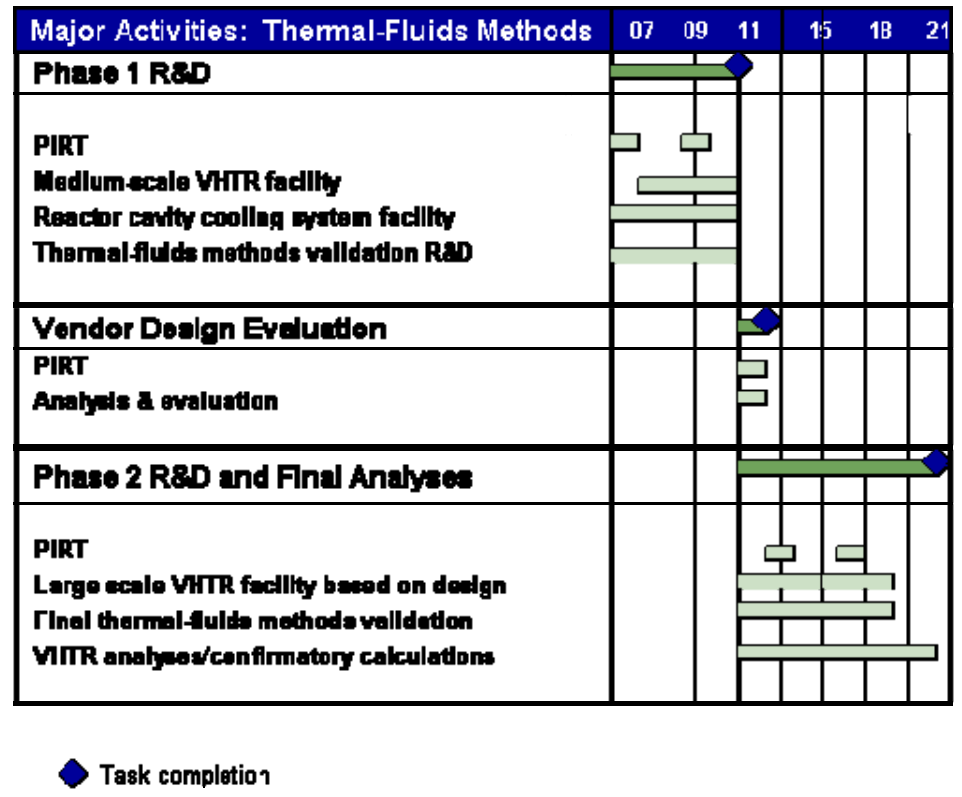

Figure 42. Timeline for thermal-fluid and structures thermal analysis 


\begin{tabular}{|c|lll|}
\hline NEXT GENERATION NUCLEAR PLANT & Identifier: & PLN-2498 & \\
METHODS RESEARCH AND & Revision: & 1 & \\
DEVELOPMENT TECHNICAL & Effective Date: & $9 / 25 / 08$ & Page: 116 of 188 \\
PROGRAM PLAN & & & \\
\hline
\end{tabular}

The following approach is being used to define and plan Methods R\&D for both Phase 1 and Phase 2:

- $R \& D$ Based on the Latest PIRT. The specified $\mathrm{R} \& \mathrm{D}$ is based on the latest phenomena identification and ranking. The PIRT used to define the R\&D is described in Appendix A together with a short description of the candidate VHTR designs. As the design of the VHTR matures, an increasingly sophisticated PIRT will be required to identify the key scenarios and important phenomena to match specifics characteristic of the chosen design. Hence the R\&D plan is based on the assumption that an ever-improving PIRT will be available. Clearly, all phenomena that must be calculated have not yet been identified even though a sufficient phenomena base has been identified to enable the methods R\&D program to be mapped and planned. A formal PIRT should be created in conjunction with the preconceptual design in approximately 2007 or 2008 and then updated as the conceptual design, the preliminary design, and final designs are formulated.

- Software Validation. The software used to analyze VHTR behavior must be validated for the scenarios and phenomena of importance. The validation process has already begun using existing data. If existing data are not available or adequate to cover the VHTR's operational, off-normal, and accident envelopes, experiments must be defined and built and data must be produced to provide the basis for software validation. To date, approximately six to eight experiments have been identified that will either require new construction or modification of existing facilities to meet known needs. Once the required data are available, a large validation effort is required for all the software tools: systems analysis and CFD coupled to neutronics tools, and software to calculate fission product tracking if the fuel fails.

- Software Development. If the validation studies show that the software cannot adequately calculate the key phenomena in the important plant scenarios, development to improve the software must be completed or more sophisticated software must be used if available or developed if not available. At present, a limited amount of software development is deemed necessary.

- Analyses. Once the software has been validated and shown to be capable of calculating the important phenomena to the accuracy required, best-estimate analysis may begin.

Both the experimental research and software-directed research areas are focused on the high-priority R\&D areas identified in the first-cut PIRT, where key regions of concern are identified. In each case, the issues are whether the system will survive, particularly under the most challenging accident conditions, and whether the system will have an adequate operational lifetime for the conditions that are postulated (rated operational conditions, off-normal operational conditions, and accident conditions). The highpriority research areas include: core heat transfer, mixing in the upper plenum, lower plenum, hot duct, and turbine inlet, heat transfer in the RCCS, air ingress following a system depressurization, and behavior of the integral system during key scenarios, including the balance-of-plant contributions. 


\begin{tabular}{|c|lll|}
\hline NEXT GENERATION NUCLEAR PLANT & Identifier: & PLN-2498 \\
METHODS RESEARCH AND & Revision: & 1 & \\
DEVELOPMENT TECHNICAL & Effective Date: & $9 / 25 / 08$ & Page: 117 of 188 \\
PROGRAM PLAN & & & \\
\hline
\end{tabular}

\section{SUMMARY}

Methods R\&D projects have been initiated and this technical program plan has been prepared to support the effort required to ensure the necessary software tools are ready to support the VHTR design effort as required by the Energy Policy Act of 2005. This plan outlines the R\&D needed to ensure that the software tools can perform the required calculations, thus showing that the VHTR can operate safely at all conditions in its operational, off-normal, and accident envelopes and operate economically for its prescribed lifetime. The overall timeline for NGNP Methods R\&D is shown in Figure 43.

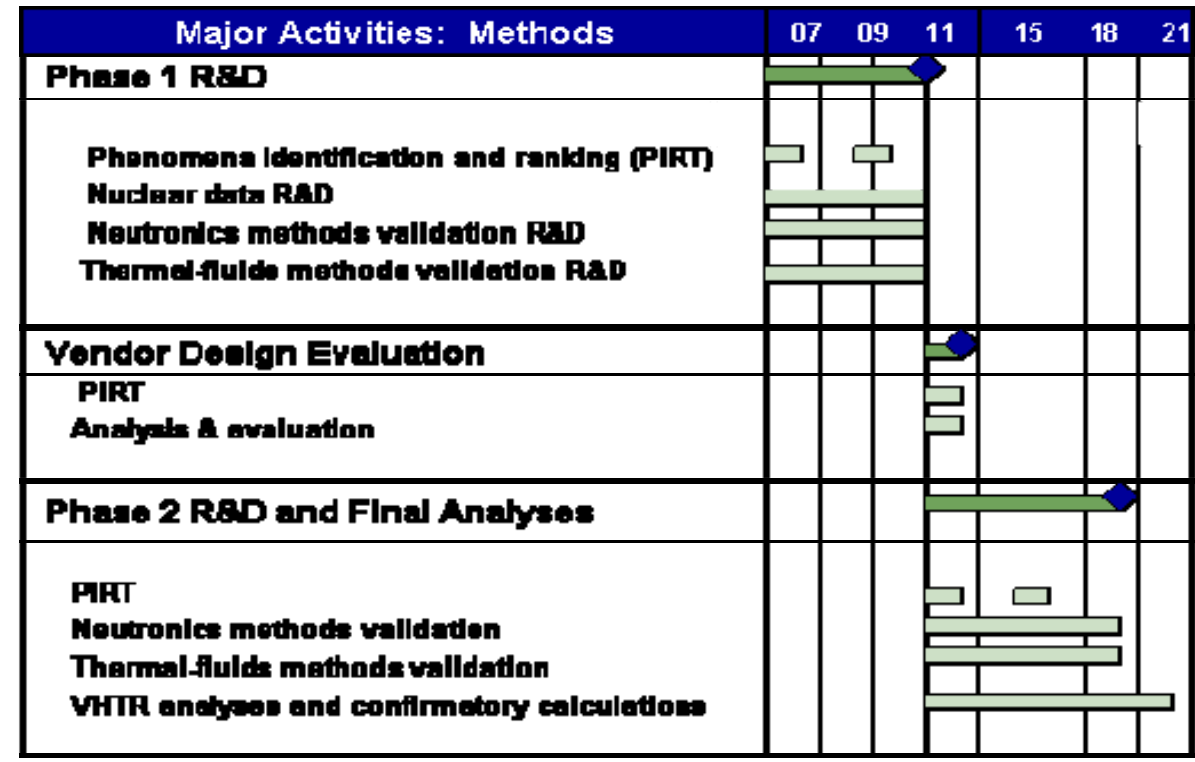

Tagk completion

Figure 43. Methods R\&D Timeline

In essence, Methods R\&D is predicated on the assumption that the software tools required to assess various proposed VHTR designs must be ready for validation and qualification when the NGNP design is selected. Methods R\&D prior to the design selection is Phase 1; thereafter, Phase 2 efforts will focus on performing experiments and calculations that demonstrate that the VHTR operational and accident characteristics are as described by the vendor. The total cost of required Methods R\&D is approximately $\$ 120 \mathrm{M} .^{\mathrm{r}}$

NGNP Methods R\&D is specifically designed to fill a gap that is not included in any of the vendor $R \& D$ efforts. The needs required create a clear niche for Methods R\&D at the national laboratories.

\subsubsection{Need for NGNP Methods}

Online nuclear power plants can only operate within limits defined by the capability of the licensee to demonstrate that all important figures-of-merit for plant safety parameters are never challenged (that is, a sufficient margin must be observed so the figures-of-merit are not in danger of being violated).

r. The $\$ 120 \mathrm{M}$ is required to complete the entire scope of work outlined in the NGNP Methods Program, including software development, benchmark activities, and actual physical experiments. 


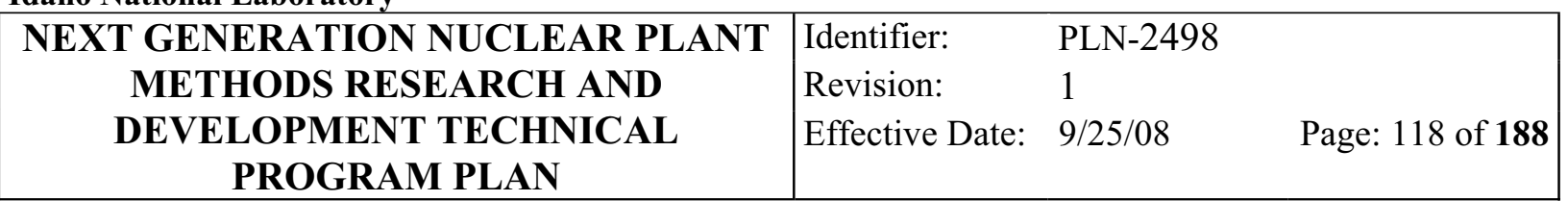

Regardless of the figure-of-merit under consideration, each has the potential to limit the operational envelope of the plant in such a way that restrictions (special precautions, operational procedures, or equipment limitations for example) will be required to ensure the figure-of-merit is not challenged. In some cases, the restrictions translate to operating the plant at power levels less than the designed power level. In some cases, the restrictions may translate to reducing the rate-of-change of an operational parameter in going from one condition to another, thus increasing the operational transit time. In any case, such restrictions have an economic penalty. It is the function of "methods" to provide the capability and the confidence for the NGNP to operate at its design limits, with maximum efficiency and economy, and at competitive $\$ / \mathrm{kW}$ rates in today's market.

\subsubsection{The VHTR Analysis Challenge}

Bulk outlet temperatures for the VHTR must be in excess of $900^{\circ} \mathrm{C}$ and as close to $1000^{\circ} \mathrm{C}$ as possible to enable the system to operate at its maximum thermodynamic efficiency and provide an optimal heat source to satisfy the NGNP mission requirements (to generate process heat or generate hydrogen). Operating at bulk outlet temperatures in excess of $900^{\circ} \mathrm{C}$ and as close to $1000^{\circ} \mathrm{C}$ as possible is what is meant by having the VHTR meet Generation IV plant operating requirements.

Peak exit gas temperatures at particular outlet jet locations for bulk outlet temperatures of $950^{\circ} \mathrm{C}$ may be considerably above $1000^{\circ} \mathrm{C}$ at operational conditions, depending on the core power distribution and the core bypass. Subsequent mixing in the outlet plenum allows for the reduction of temperature in the hottest plumes down to the bulk average temperature, but the local gas jets exiting the core have the potential to impinge on the walls of the flow path and create localized hot spots or excessive temperature gradients, unless the design has properly accounted for such behavior. This high temperature gas jet problem impacts materials placement and selection for the accident scenarios and operational plant considerations. Similar arguments apply to conditions that may exist for the transient scenarios and the most challenging accident scenarios.

\subsubsection{Mission of NGNP Methods}

The mission of NGNP Methods is to develop and benchmark state-of-the-art analysis tools that will enable vendors, the NRC, and DOE analysts to accurately calculate the core power distribution, core bypass, and peak outlet jet temperatures such that localized hot spots can be identified and either eliminated by design or quantified to a degree that licensing calculations can demonstrate VHTR plant operation without challenging the safety margins, plant parameters, or figures-of-merit. Advanced tools for analyzing the VHTR are consistent with new safety concerns inherent in the new design and new system design requirements. Thus, examples of products that will be provided by the NGNP Design and Safety Methods Validation Project are software tools and the pertinent experiments for the validation or benchmarking of these tools that will allow the plant operational and licensing requirements to be calculated.

For operational conditions, specific analysis tools are needed to calculate:

- The prescribed distribution of the fuel in the core, both initially and at any time during the life of the reactor. The fuel in the prismatic reactors is fixed, but the capability to define where each fuel element should be located (given a defined enrichment), both initially and during reloads, is essential as is the capability to calculate the burnup histories of each fuel element over its tenure in its prescribed location. The fuel in the PBR begins its journey through the core as a function of where it is initially placed at the top of the core; the trajectory of the pebbles and how they influence the core power at each point along their paths must be calculated. 
NEXT GENERATION NUCLEAR PLANT METHODS RESEARCH AND DEVELOPMENT TECHNICAL PROGRAM PLAN
Identifier: $\quad$ PLN-2498

Revision: 1

Effective Date: 9/25/08

- The 3-D core power distribution.

- The helium coolant core flow distribution - including the bypass flows.

- The flow distribution and flow dynamics throughout the remainder of the reactor vessel, for example in the outlet plenum.

- The potential for cyclic flows and/or equipment flutter and vibration.

- How to prescribe fuel enrichments and locations for reloads.

- The graphite dust generation rate.

- Graphite dust distribution throughout the system.

- Whether localized hot spots exist throughout the system that may lead to premature structural failures.

- Whether excessive thermal gradients are present that may lead to premature structural failures.

- The reactor vessel wall temperature distribution and the interaction between the reactor and the reactor cavity.

- The parasitic energy losses to the reactor cavity and the cavity cooling system.

- The inlet, in-component, and exit conditions (most likely 2-D or 3-D) for the plant power conversion systems, for example an IHX.

For off-normal and accident conditions, specific analysis tools are needed to calculate:

- The leakage rates for various break configurations and the resulting effects on the system such as (a) graphite dust redistribution, both within the reactor and through the leak to the reactor cavity, (b) pressure pulse propagation, (c) structural loads, (d) depressurization rates, (e) flow distributions within the reactor system resulting from prolonged leaks, and (f) the effect of the leaks on the fluid dynamics and pressurization rates in the reactor cavity.

- Potential reactivity events, including the fuel power transients and reactivity interactions with the system.

- The fuel temperatures and fuel failure rates.

- The core temperature distribution throughout the various off-normal and accident scenarios.

- Density-gradient flow distributions both for depressurized conduction cooldown and pressurized conduction cooldown scenarios, including the presence of localized hot spots and potentially excessive thermal gradients.

- The stratified flow behavior that will occur when a break unchokes and the flow from the vessel and the flow into the vessel are governed by the density differences between the outgoing helium and incoming air - for densimetric Froude numbers less than one.

- The air ingress into the core region, including the extent of graphite oxidation that will occur.

- The fluid dynamics of potential water ingress scenarios.

- The energy transfer between the reactor (and any discharge from the reactor) to the reactor cavity and the cavity cooling systems. 


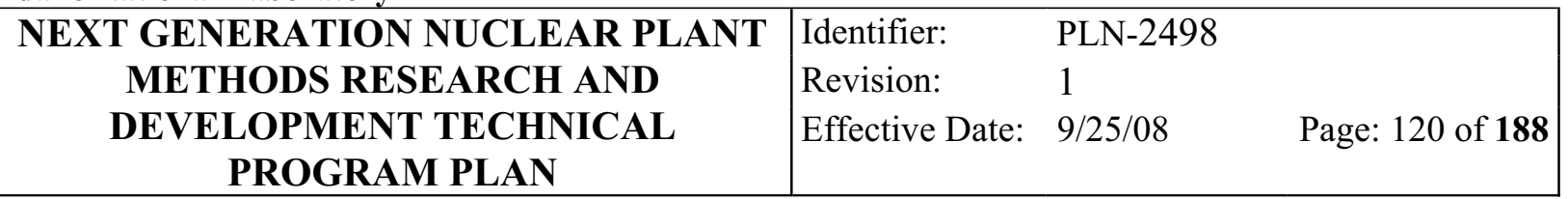

- The potential for boiling and dryout of the cavity cooling working fluid (two of the candidate designs use water to cool the reactor cavity walls).

- The trajectories of the released fission products and the fraction of the fission products that are released to the out-plant environment.

- The reactor system behavior during seismic events.

\subsubsection{Advantages of Advanced Analysis Tools}

Advanced analysis tools offer the following advantages over older legacy analysis tools:

- Reduced design uncertainty and risk because the localized conditions can be calculated with a known uncertainty.

- Reduced design iterations and design costs because the plant designers can more rapidly converge on their desired design configuration with less uncertainty.

- An accelerated licensing process because the process of prescribing arbitrary safety factors is transformed to a process of using known quantities with quantified uncertainties.

- The capability of quantifying the safety and operational margins to optimal values for maximum outlet temperatures and maximum operational efficiencies.

\subsubsection{The National Laboratory Niche in Methods}

The gas-cooled thermal reactors built and operated in the United States and elsewhere to date are characterized by sustained operations at conditions that provide or have substantial design and safety margins. The margins were designed to be large because the legacy analysis tools used to calculate limiting parameters on the systems to date were not capable of calculating important local limiting parameters with sufficient accuracy to reduce the safety margins to more desirable levels such that the economics of the plant operational, off-normal, and accident envelopes can be optimized. These practices were used because, until recently, analysis tools and the required boundary conditions (material properties and neutronics cross-sections) were not available such that the required parameters (for example localized power distributions and localized wall temperatures) could be calculated with a sufficiently low calculational uncertainty.

The commercial companies (Areva, Ltd, General Atomics, PBMR Pty, etc.) currently designing the future gas-cooled reactors are still, in large measure, using legacy analysis tools to describe the operating and accident characteristics of their designs. And they intend to use them for licensing purposes. Recent visits by NGNP Project personnel to the headquarters of Areva Ltd and PBMR Pty have allowed important interactions between the commercial researchers and the national laboratory researchers to occur. It is clear, based on these interactions, that the products of the NGNP Design and Safety Methods Project will be useful to the vendors.

The NGNP Methods development program will provide advanced, state-of-the-art tools that are independent of those used by the vendors. Of these tools, most of them are already available and they consist of a mixture of commercial software and software written at the national laboratories. Only limited tool development is necessary for NGNP methods. Therefore, the majority of the NGNP Methods Program (in terms of cost) is focused on software V\&V. The cost distribution is projected to be approximately $\$ 120 \mathrm{M}$ of which $16 \%$ is for neutronics and $84 \%$ is for thermal-fluids. The cost distribution projections are greatly influenced by the cost of the required experiments. Once V\&V of the NGNP 


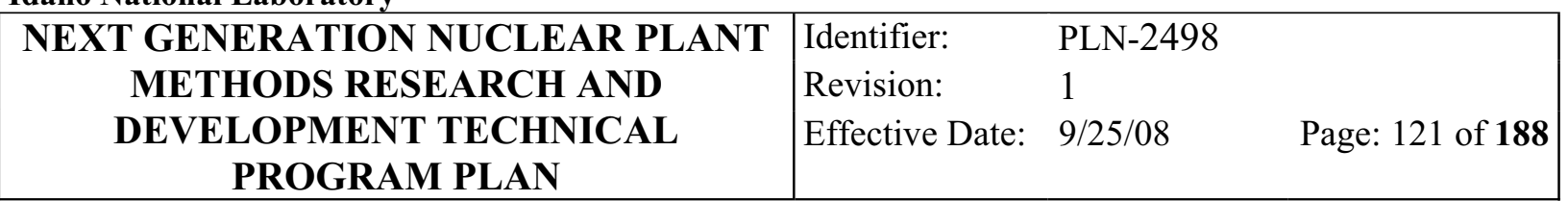

software is completed, the NGNP Methods-generated tools may be used by the vendors and will be available for use by the DOE and the NRC.

Although it is projected that $\$ 120 \mathrm{M}$ is required to complete the entire scope of work outlined in the NGNP Methods Program including software development, benchmark activities, and actual physical experiments, the cost will be more than recovered by the capability of the plant to operate at peak thermal efficiencies with maximum outlet temperatures.

\subsubsection{NGNP Methods Program}

The calculational envelope of the neutronics and thermal-fluids software tools intended to be used on the NGNP is defined by the scenarios and phenomena that these tools can calculate with confidence. The software tools can only be used confidently when the results they produce have been shown to be in reasonable agreement with first-principle results, thought-problems, and data that describe the highlyranked phenomena inherent in all operational conditions and important accident scenarios for the VHTR.

The VHTR design has not yet been selected. Consequently, the R\&D process is focused on scenarios and highly-ranked phenomena that have already been identified as important by the advanced gas-cooled reactor community for the designs being considered as candidates for the VHTR. This approach has resulted in a VHTR-specific PIRT from which the methods R\&D is being defined using the following assumptions:

1. The selected VHTR design could be either a pebble-bed or a block-type reactor.

2. The calculational and experimental needs, and consequently the required $R \& D$, is focused in eight distinct areas based on the relative state of the software in each. The areas are

a. Basic differential and integral nuclear cross-section data measurement and evaluation, including mathematically rigorous sensitivity studies of the effects of uncertainties in the differential nuclear data and other independent design variables on key integral reactor properties (the task of characterizing the effects of the nuclear fuel, fission products, moderator, and other relevant materials on the system reactivity, neutron flux distribution, and power production)

b. Reactor assembly cross-section preparation (the task of translating the fundamental data characterized in area (i) into formats and states useful for analysis)

c. Discrete ordinates transport (the process of approximating the neutron flux in a tractable manner for analysis)

d. Nodal diffusion (calculation of the energy and spatial flux profiles, reaction rates, reactivity changes, etc.)

e. Reactor kinetics (calculation of spatial changes in flux and power level as functions of time during postulated transients)

f. Thermal-fluids (the models that describe the fluid behavior and heat transfer behavior during steady-state and transient conditions for the scenarios of interest)

g. Fuel behavior (this R\&D area is being accomplished in the Advanced Gas Reactor Program) 


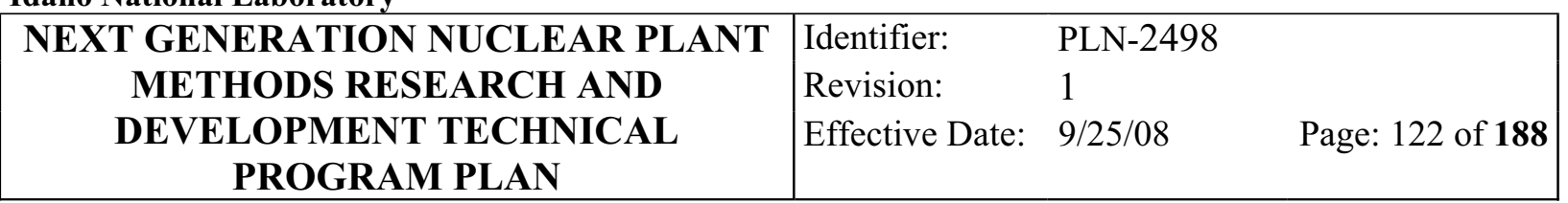

h. Fission product transport (determination of fission product movement once fission products have escaped from the confines of the fuel).

For FY 2007 and 2008 the highest-priority R\&D is aimed at properly calculating the thermal-fluid conditions in the lower plenum during normal operation, developing and validating neutronics techniques that are necessary for analyzing both prismatic and PBRs, and analyzing the behavior of the plant during DCC and PCC accident scenarios.

Subsequent years will sharpen the focus in these and other areas that require analyses with low uncertainties for the most challenging scenarios identified by the PIRTs. The PIRTs form the heart of the Methods R\&D effort in that the R\&D needs are both identified and prioritized. Hence the PIRTs used to govern the R\&D needs will be updated throughout the cycle leading to the construction of the VHTR at INL.

To demonstrate whether or not the NGNP analysis software is capable of analyzing the above phenomena and plant integral behavior, a set of data are required for almost every item identified for operational conditions, off-normal conditions, and accident conditions to allow a rigorous validation to be performed. In some cases the data are available, but need to qualified. However, there are a considerable number of phenomena or plant integral behavior scenarios that require new experiments to be performed. 


\begin{tabular}{|c|lll|}
$\begin{array}{c}\text { NEXT GENERATION NUCLEAR PLANT } \\
\text { METHODS RESEARCH AND }\end{array}$ & Identifier: & PLN-2498 \\
Revision: & 1 & \\
DELOPMENT TECHNICAL & Effective Date: & $9 / 25 / 08$ & Page: 123 of 188 \\
PROGRAM PLAN & & & \\
\hline
\end{tabular}

\section{REFERENCES}

American Nuclear Society, 2003, American Nuclear Society MHTGR Technology Course, June 2003.

Auchampaugh, G. F., J. A. Farrell, and D. W. Bergen, 1971, "Neutron-induced fission cross-sections of ${ }^{242} \mathrm{Pu}$ and ${ }^{244} \mathrm{Pu}, " N u c l$. Phys. A171, 31.

Babcock, R. S., D. E. Wessol, C. A. Wemple, and S. C. Mason, "The MOCUP Interface: A Coupled Monte Carlo/Depletion System," 1994 Topical Meeting on Advances in Reactor Physics, Knoxville, TN, p. III-368 (April 11-14, 1994). Available as RSICC package PSR-0365/02.

Ball, S. J., 2003, "MHTGR Accident Analysis," American Nuclear Society MHTGR Technology Course, June 2003.

Ball, S. J., 2004, Personal communication.

Ball, S. J., T. D. Burchell, W. R. Corwin, S. E. Fisher, C. W. Forsberg, R. N. Morris, D. L. Moses, 2008, Next Generation Nuclear Plant Gap Analysis Report, ORNL/TM-2007/228, July 2008.

Bergen, D. W., and R. R. Fullwood, 1971, "Fission cross-sections of ${ }^{242} \mathrm{Pu}$," Nucl. Phys. A163, 577.

Bernard, P. S., J. D. Crouch, M. Choudhari, D. G. Bogard, and K. A. Thole, 1998, "Transition and Turbulence," The Handbook of Fluid Dynamics, R. W. Johnson, ed, CRC.

Boyack, B. E., et al., 1990, "Quantifying Reactor Safety Margins Part 1: An Overview of the Code Scaling, Applicability, and Uncertainty Evaluation Methodology," Nuclear Engineering and Design, Vol. 119, pp. 1-15.

Briewsmeister, J. F., Editor, 1986, "MCNP-A General Purpose Monte Carlo Code for Neutron and Photon Transport," LA-7396-M, Rev 2, September 1986.

Budtz-Jorgensen, C., H. H. Knitter, 1981, "Neutron Induced Fission Cross-section of Pu-240 in the Energy Range from $10 \mathrm{kev}$ to $10 \mathrm{Mev}$," Nucl. Eng. Sci., Vol 79, p. 380.

Byers, D. H., B. C. Diven, and M. G. Silbert, 1966, "Capture and Fission Cross-section of ${ }^{240} \mathrm{Pu}$," 66WASH, 903.

Cappiello, M., Los Alamos National Laboratory, private communication to R.G. Bennett, INEEL, 2004, "Review of IPNS Proposal," May (AFC National Technical Director for Transmutation).

Cochran, P., P. Saha, P. Hejzlar, D. McEligot, G. E. McCreery, and R. Schultz, 2004, Scaling Analysis and Selection of Test Facility for Facility for Fundamental Thermal-Hydraulic Studies Related to Advanced Gas-Cooled Reactor, INEEL/EXT-04-02084, September 2004.

Cogliati, J. J., and A. M. Ougouag, "PEBBLES: A Computer Code for Modeling Packing, Flow and Recirculation of Pebbles in a Pebble Bed Reactor," accepted for publication at HTR 2006, 3rd International Topical Meeting on High Temperature Reactor Technology_HTR 2006, paper C00000180, October 1-4, 2006, Sandton, Johannesburg, South Africa.

Croff, A. G., 1980, ORIGEN2-A Revised and Updated Version of the Oak Ridge Isotope Generation and Depletion Code, ORNL-5621, Oak Ridge National Laboratory, July 1980.

Fletcher, C. D., P. D. Bayless, C. B. Davis, M. G. Ortiz, S. M. Sloan, R. A. Shaw, R. R. Schultz, C. E. Slater, G. W. Johnsen, J. P. Adams, and L. S. Ghan, 1997, Adequacy Evaluation of RELAP5/MOD3, Version 3.2.1.2 for Simulating AP600 Small Break Loss-of-Coolant Accidents, INEL-96/0400, April 1997. 


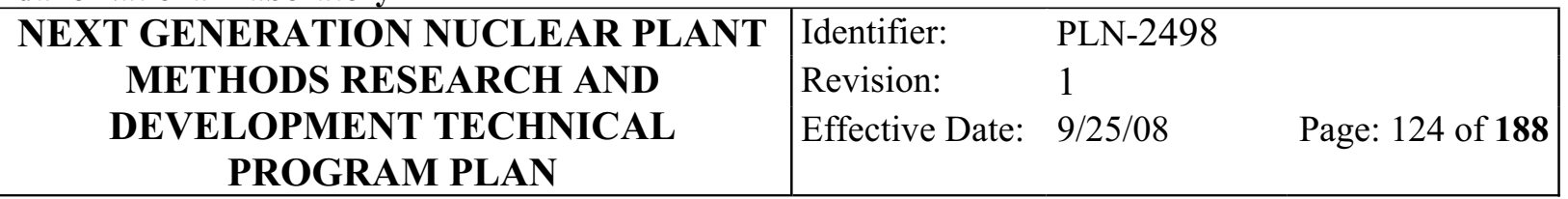

Freitas, C. J., U. Ghia, I. Celik, H. Coleman, P. Raad, and P. J. Roache, 2003, “ASME's Quest to Quantify Numerical Uncertainty," AIAA Paper 2003-0627, AIAA 41st Aerospace Sciences Meeting, Reno, NV, January 2003.

General Atomics Co., 1976, Fort Saint Vrain Final Safety Analysis Report.

Generation IV International Forum, 2002, “A Technology Roadmap for Generation IV Nuclear Power Systems,” GIF-002-00, December 2002.

Gougar, H. D., A. M. Ougouag, W. K. Terry, and K. N. Ivanov, 2004, "Design of a Very High Temperature Pebble-Bed Reactor Using Genetic Algorithms," in PHYSOR 2004 -The Physics of Fuel Cycles and Advanced Nuclear Systems: Global Developments, Chicago, Illinois, April 25-29, 2004, on CD-ROM, American Nuclear Society, Lagrange Park, IL.

Grimesy, R. A., D. W. Nigg, and R. L. Curtis, 1991, COMBINE/PC-A Portable ENDF/B Version 5 Neutron Spectrum and Cross-Section Generation Program, EGG-2589, Revision 1, Idaho National Engineering Laboratory, February 1991.

Hockenbury, R. W., A. J. Sanislo, and N. N. Kaushal, 1975, "keV capture cross-section of ${ }^{242} \mathrm{Pu}$,"Conf. Nuclear Cross-sections Technology, 2, 584, Washington.

Hudson, N., G. Ilas, F. Rahnema, A. Ougouag, H. Gougar, W. Terry and R. Mphahlele, "Accuracy of MICROX-2 for PBR Analysis Using Monte Carlo Technique," in proceeding of The Monte Carlo Method: Versatility Unbounded in a Dynamic Computing World, Chattanooga, Tennessee, April 1721, 2005, on CD_ROM, American Nuclear Society, LaGrange Park, IL (2005).

IAEA, 2003, Evaluation of high temperature gas cooled reactor performance: Benchmark analysis related to initial testing of the HTTR and HTR-10, IAEA-TECDOC-1382.

IAEA, 2000, Heat Transport and Afterheat Removal for Gas Cooled Reactors Under Accident Conditions, IAEA-TECDOC-1163.

IAEA, 1997, Fuel Performance and fission product behavior in gas cooled reactors, IAEA-TECDOC978.

Johnson, R. W., R. R. Schultz, P. J. Roache, I. B. Celik, W. D. Pointer, and Y. A. Hassan, 2006, Processes and Procedures for Application of CFD to Nuclear Reactor Safety Analysis, INL/EXT-0611789, September 2006.

Kashiwa, B. A., N. T. Padial, R. M. Rauenzahn, and W. B. VanderHeyden, 1993, A Cell-Centered ICE Method for Multiphase Flow Simulations, LA-UR-93-3922.

Kashiwa, B. A., and R. M. Rauenzahn, 1994, A Multimaterial Formalism, LA-UR-94-771.

Kayes, W. M., and M. E. Crawford, Convective Heat and Mass Transfer, Second Edition, McGraw-Hill Book Company, New York, 1980.

Keller, S., F. Rahnema, C. de Oliveira, Matthew Eaton, A. M. Ougouag, W. K. Terry, and H. D. Gougar, 2002, "Evaluation of Cross Section Processing Codes COMBINE and WIMS for Pebble Bed Reactor Fuel Cycle Analysis" Proceedings of PHYSOR-2002, Seoul, South Korea, October 2002.

Krüger, K., A. Bergerfurth, S. Burger, P. Pohl, M. Wimmers, and J. C. Cleveland, 1991, "Preparation, Conduct, and Experimental Results of the AVR Loss-of-Coolant Accident Simulation Test," Nuclear Science and Engineering, 107, pp. 99-113.

Kunitoni, K., Y. Inagaki, I. Ioka, Y. Kondo, Y. Miyamoto, H. Shimomura, T. Akisada, and S. Yamaguchi, 1986, Experience Obtained from Construction and Preliminary Test of In-core Structure Test Section, JAERI-M 86-192. 


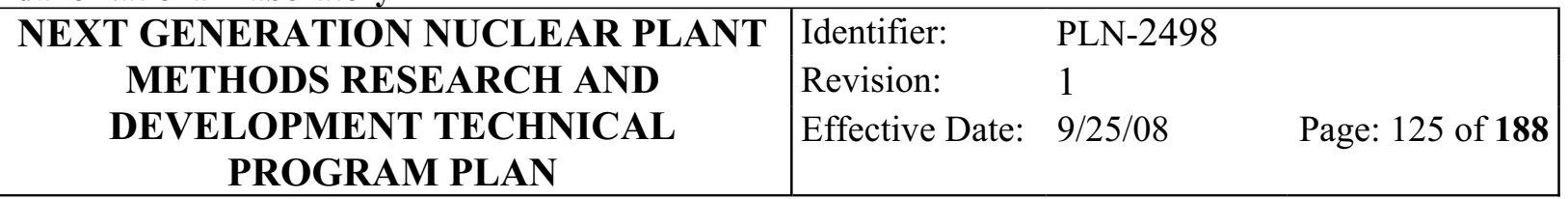

Lee, C. H., Z. Zhong,, T. Taiwo, W. Yang, M. Smith, and G. Palmiotti, 2006, Status of Reactor Physics Activities on Cross Section Generation and Functionalization for the Prismatic Very High Temperature Reactor, and Development of Spatially-Heterogeneous Codes, ANL-GenIV-075, ANL, August 2006.

Lee, C. H., Z. Zhong, T. Taiwo, W. Yang, and M. Smith, 2006, Enhancement of REBUS-3/DIF3D for Whole-Core Neutronics Analysis of Prismatic Very High Temperature Reactor (VHTR), ANLGenIV-076, September 2006.

Lee, W. J., T. Y. C. Wei, and R. R. Schultz, 2005, et al, Generation of a Preliminary PIRT (Phenomena Identification and Ranking Table) for Very High Temperature Gas-Cooled Reactors, KAERI/TR3050/2005, INL/EXT-05-00829, ANL-GenIV-066, September 2005.

MacDonald, P. E., et al., 2003, NGNP Preliminary Point Design-Results of the Initial Neutronics and Thermal-Hydraulic Assessments, INEEL/EXT-125-00870 Rev. 1, September 2003.

MacDonald et al 2003 (paper on point design) P. E. MacDonald, P. D. Bayless, H. D. Gougar, R. L. Moore, A. M. Ougouag, R. L. Sant, J. W. Sterbentz, and W. K. Terry, "The Next Generation Nuclear Plant - Insights Gained from the INEEL Point Design Studies" Paper 4305, pp. 349-363, in Proceedings of ICAPP-04, Pittsburgh, PA, USA, June 13-17, 2004.

Marleau et al 2008: G. Marleau, A. Hebert and R. Roy, 2008, A User Guide for DRAGON Version 4, Technical Report IGE-294, Institut de g'enie nucl'eaire D'epartement de g'enie m'ecanique 'Ecole Polytechnique de Montr'eal, August 13, 2008.

Mercatali, L., et al., 2004, "Irradiation Experiment Analysis for Cross-section Validation," PHYSOR 2004 - The Physics of Fuel Cycles and Advanced Nuclear Systems: Global Developments, Chicago Illinois, April 25-29, American Nuclear Society, Lagrange Park, IL.

Migneco, E., and J. P. Theobald, 1968, "Resonance Grouping Structure in Neutron Induced Subthreshold Fission of 94-PU-240," Nucl. Phys. Vol A112, p. 603.

Miller, G. K., D. A. Petti, J. T. Maki, and D. L. Knudson, 2004, "Current Capabilities of the Fuel Performance Modeling Code PARFUME," Proceedings of the High Temperature Reactor Conference HTR-2004, Beijing, China, September 2004.

Moorman, R., 2008, A Safety Re-Evaluation of the AVR Pebble Bed Reactor Operation and Its Consequences for Future HTR Concepts, Berichte des Forschungszentrums Jülich, 4275, IISSN 0944-2952.

Morris, R., D. Petti, D. Powers, and B. Boyack, 2004, “TRISO Coated Particle Fuel Phenomena Identification and Ranking Tables (PIRT) for Fission Product Transport Due to Manufacturing, Operations, and Accidents," NUREG/CR-6844, July 2004.

OECD-NEA Nuclear Science Committee, 2006, "International Handbook of Evaluated Reactor Physics Benchmark Experiments," NEA/NSC/DOC(2006)1, March 2006.

Ougouag, A. M., H. D. Gougar, W. K. Terry, R. Mphahlele, and K. N. Ivanov, 2004, "Optimal Moderation in the Pebble-Bed Reactor for Enhanced Passive Safety and Improved Fuel Utilization" in PHYSOR-2004 - The Physics of Fuel Cycle and Advanced Nuclear Systems: Global Developments, Chicago, Illinois, April 25-29, 2004, on CD-ROM, American Nuclear Society, Lagrange Park, IL.

Ougouag, A. M., Hans D. Gougar, William K. Terry. Frederik Reitsma and Wessel R. Joubert, 2005, "PEBBED Analysis of Hot Spots in Pebble-Bed Reactors," Mathematics and Computation, Supercomputing, Reactor Physics and Nuclear and Biological Applications, Palais des Papes, Avignon, France, September 12-15, 2005, on CD-ROM, American Nuclear Society, LaGrange Park, IL (2005). 


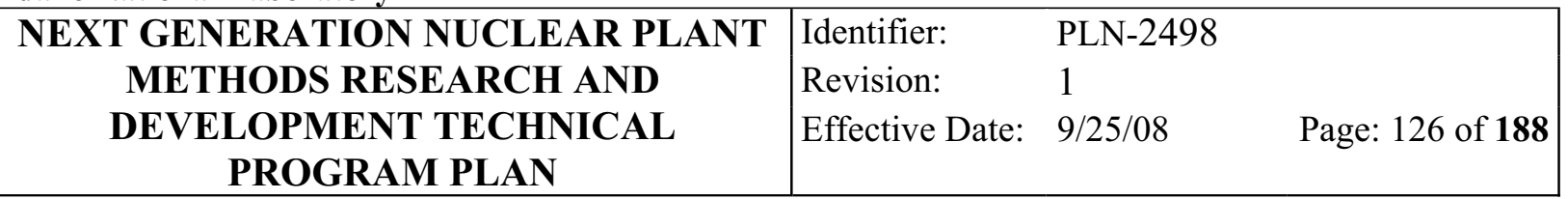

Ougouag, A. M., Jan Leen Kloosterman, Wilfred F. G. van Rooijen, Hans D. Gougar, and William Kerry, 2006, "Investigation of bounds on particle packing in pebble-bed high temperature reactors," Nuclear Engineering and Design, Vol 236, pp. 669-676.

Ougouag, A. M., and J. J. Cogliati, 2007, "Earthquakes and Pebble Bed Reactors: Time-Dependent Densification," published in Transactions of Joint International Topical Meeting on Mathematics \& Computation and Supercomputing in Nuclear Applications (M\&C + SNA 2007) Monterey, California, April 15-19, 2007, on CD-ROM, American Nuclear Society, LaGrange Park, IL (2007).

Ougouag, O. M., William K. Terry and Hans D. Gougar, 2008, “Optimally Moderated Nuclear Fission Reactor and Fuel Source Therefore, United State Patent US 7,403,585 B2, July 22, 2008.

Oak Ridge National Laboratory, 1999, MICROX-2, Code System to Create Broad-Group Cross Sections with Resonance Interference and Self-Shielding from Fine-Group and Pointwise Cross Sections, PSR-374, Oak Ridge National Laboratory, January 1999.

Petti, D., R. Hobbins, J. Kendall, and J. Saurwein, 2005, Technical Program Plan for the Advanced Gas Reactor Fuel Development and Qualification Program, INL/EXT-05-00465, August 2005.

Potter, and A. Shenoy, 1996, Gas Turbine-Modular Helium Reactor (GTMHR) Conceptual Design Description Report, GA Report 910720, Revision 1, General Atomics, July 1996.

Public Law 109-58, 2005, “Energy Policy Act of 2005," 119 Stat. 594, $109^{\text {th }}$ Congress, Aug 8, 2005.

Roache, P. J., 1998, Verification and Validation in Computational Science and Engineering, Hermosa Publishers, Albuquerque.

Schmidt, F.W., R. E. Henderson, and C. H. Wolgemuth, 1993, Introduction to Thermal Sciences, 2nd ed., John Wiley and Sons, pp. 248-252.

Schultz, R. R., S. J. Ball, and J. King, 2004, Catalogue of Validation Data for Gas-Cooled Reactor Operational and Accident Scenarios, INEEL/EXT-04-02294, September 2004.

Schultz, R. R., 1993, International Code Assessment and Applications Program: Summary of Code Assessment Studies Concerning RELAP5/MOD2, RELAP5/MOD3, and TRAC-B, NUREG/IA-0128, December 1993.

Schultz, R. R., R. A. Riemke, and C. B. Davis, 2003, "Comparison: RELAP5-3DC systems analysis code and Fluent CFD code momentum equation formulation," Proceedings of ICONE11: 11th International conference on Nuclear Engineering, Tokyo, Japan, April 20-23, 2003.

Schultz, R. R., H. Gougar, and T. Y. C. Wei, 2006, Conversations with PBMR personnel, March 2006.

Schultz, R. R., T. Taiwo, W. K. Terry, and T. Y. C. Wei, 2006, Conversations with Areva personnel, July 2006.

Speziale, C. G., and R. M. C. So, 1998, "Turbulence Modeling and Simulation," The Handbook of Fluid Dynamics, R. W. Johnson, ed, CRC.

Sterbentz, J. W., 2002, Uranium and Plutonium Isotopic Validation Study for the Hanford Reactor, INEEL/EXT-02-01567.

Sterbentz, J. W., C.A. Wemple, 1996, Calculation of a Burnup Methodology and Validation for the Idaho National Engineering Laboratory Spent Nuclear Fuels, INEL-96/0304.

Taiwo, T. A., W. S. Yang, S. J. Kim, T. K. Kim, and Mark Paulson, 2005, Evaluation of the Compact Nuclear Power Source (CNPS) Critical Experiments, ANL-Gen-IV-059, September 2005.

Taiwo, T. A., personal communication with A. M. Ougouag, August 8, 2008. 


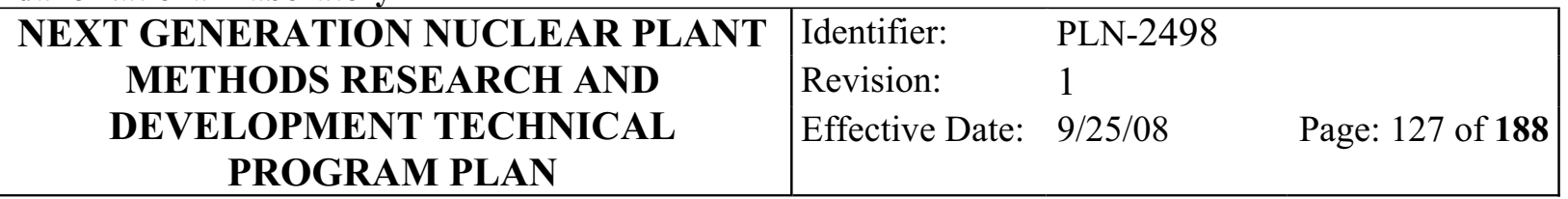

Terry, W. K., H. D. Gougar, and A. M. Ougouag, 2002, "Direct Deterministic Method for Neutronics Analysis and Computation of Asymptotic Burnup Distribution in a Recirculating Pebble-Bed Reactor," Annals of Nuclear Energy, Vol 29, pp. 1345-1364.

Terry, W. K., J. K. Jewell, and J. Blair Briggs, T. A. Taiwo, W. S. Park and H. S. Khalil, 2004, "Preliminary Assessment of Existing Experimental Data for Validation of Reactor Physics Codes and Data for NGNP Design and Analysis," September 2004.

Teuchert E., U. Hansen, K. A. Haas, H. J. Rutten, H. Brockmann, H. Gerwin, U. Ohlig, and W. Scherer, 1980, V.S.O.P.—Computer Code System for Reactor Physics and Fuel Cycle Simulation, KFA-IRE Report Jül-1649.

Teuchert, E., U. Hansen, K. A. Haas, H. J. Rutten, H. Brockmann, H. Gerwin, U. Ohlig, and W. Scherer, "V.S.O.P. Computer Code System for Reactor Physics and Fuel Cycle Simulation - Input Manual and Comments," April 1994.

Thomas, R. E., 1971, Chemical Aspects of MSRE Operations, ORNL-4658, Oak Ridge National Laboratory.

Toppel, B. J., 1990, The Fuel Cycle Analysis Capability REBUS3, ANL-83-2, March 1983, revised October 26, 1990, CCC-0653/02.

Turinsky, P. J., et al., 1994, NESTLE: A Few-Group Neutron Diffusion Equation Solver Utilizing the Nodal Expansion Method for Eigenvalue, Adjoint, Fixed-Source Steady-State and Transient Problems, EGG-NRE-11406.

U.S. Nuclear Regulatory Commission, 2005, Regulatory Guide 1.203, December 2005.

Vilim, R., 2004, "Initial VHTR Accident Scenario Classification: Models and Data."

Weinberg, A. M., et al., 1970, "The Status and Technology of Molten Salt Reactors-A Review of Work at the Oak Ridge National Laboratory," Nucl. Appl. Tech., Vol. 8(2), February 1970.

Weston, L. W., J. H. Todd, 1977, "Neutron Capture Cross-section of Plutonium-240," Nucl. Eng. Sci., Vol 63, p. 143.

Weston, L. W., J. H. Todd, 1984, "Subthreshold fission cross-section of Pu240 and the fission crosssections of U235 and Pu239," Nucl. Eng. Sci., Vol 88, p. 567.

Weigmann, H., J. A. Wartena, and C. Burkholz, "Neutron Induced Fission Cross-section of PU-242," Nuclear Physics.

Williams, W., P. Hejzlar, M. J. Driscoll, W-J. Lee, and P. Saha, 2003, “Analysis of a Convection Loop for GFR Post-LOCA Decay Heat Removal from a Block-Type Core,” MIT-ANP-TR-095, March 2003.

Zhong, D., Qin, Z., 2001, "Overview of the 10 MW High Temperature Gas-cooled Reactor Test Module," Proceedings of the Seminar on HTGR Application and Development, March 19-21, Beijing, China, pp. 194-206. 
NEXT GENERATION NUCLEAR PLANT

METHODS RESEARCH AND

DEVELOPMENT TECHNICAL

PROGRAM PLAN
Identifier: $\quad$ PLN-2498

Revision: $\quad 1$

Effective Date: 9/25/08 Page: 128 of $\mathbf{1 8 8}$ 


\begin{tabular}{|c|lll|}
\hline NEXT GENERATION NUCLEAR PLANT & Identifier: & PLN-2498 & \\
METHODS RESEARCH AND & Revision: & 1 & \\
DEVELOPMENT TECHNICAL & Effective Date: & $9 / 25 / 08$ & Page: 129 of 188 \\
PROGRAM PLAN & & & \\
\hline
\end{tabular}

\section{Appendix A}

\section{System Description and Phenomena Identification and Ranking Tables}


NEXT GENERATION NUCLEAR PLANT

METHODS RESEARCH AND

DEVELOPMENT TECHNICAL

PROGRAM PLAN
Identifier: $\quad$ PLN-2498

Revision: $\quad 1$

Effective Date: 9/25/08 Page: 130 of $\mathbf{1 8 8}$ 


\begin{tabular}{|c|lll|}
\hline NEXT GENERATION NUCLEAR PLANT & Identifier: & PLN-2498 \\
METHODS RESEARCH AND & Revision: & 1 & \\
DEVELOPMENT TECHNICAL & Effective Date: & $9 / 25 / 08$ & Page: 131 of 188 \\
PROGRAM PLAN & & & \\
\hline
\end{tabular}

\section{Appendix A}

\section{System Description and Phenomena Identification and Ranking Tables}

The phenomena identification and ranking tables (PIRTs) described herein were defined by the work of an International Nuclear Engineering Research Initiative (I-NERI) between Korea and the United States entitled: "Screening of Gas-Cooled Reactor Thermal-Hydraulic and Safety Analysis Tools and Experiment Database." The details of the work are given in the reference: W. J. Lee, T. Y. C. Wei, and R. R. Schultz, et al, Generation of a Preliminary PIRT (Phenomena Identification and Ranking Table) for Very High Temperature Gas-Cooled Reactors, September, 2005 (to be published).

Although formal designs have not been submitted for consideration to the DOE for the VHTR identified in the Energy Policy Act of 2005, the preliminary PIRT defined by the I-NERI effort was conceived to provide preliminary guidance to assist in the formulation of realistic R\&D plans that will lead to experiments and code development/validation that will be applicable to the final requirements of the VHTR methods project.

Because formal designs are not available for consideration, the I-NERI researchers chose to use the only information available to them regarding plants that are at least derivatives of those designs that will ultimately be proposed for the VHTR that will stem from the Energy Policy Act of 2005. An overall description of the reference prismatic and pebble-bed designs is given in the following section followed by a summary of the PIRT itself.

\section{A-1. REFERENCE GAS-COOLED VHTR DESIGNS USED FOR PIRT STUDY}

The reference gas-cooled VHTR designs are an extension of the earlier designs of the General Atomics' Gas Turbine-Modular Helium Reactor (GT-MHR) and the PBMR as marketed by PBMR Pty. The GT-MHR is a $600 \mathrm{MWth}$ direct cycle gas reactor with a prismatic core as shown in Figure A-1. The reactor operating pressure is 70 bars and the outlet temperature is $850^{\circ} \mathrm{C}$. The reference PBMR is a 400 MWth direct cycle gas reactor with a pebble-bed core as shown in Figure A-2. The reactor operating pressure is 90 bars and outlet temperature is $900^{\circ} \mathrm{C}$. The target gas-cooled VHTR differs from these designs, mainly in that the target reactor outlet temperature may be higher, although a specific value has not been defined, and the VHTR is to produce hydrogen in addition to electricity.

Both designs are assumed to have confinements. That is, the reactor cavity is vented to atmosphere if the cavity is over-pressurized. However, the vent (pressure relief valve) is fitted with a filter to minimize the release of harmful material to the environment and the pressure relief valve will close once the confinement pressure is reduced to an acceptable value. As shown in the figures, the two reactor system designs differ mainly in the core configuration, that is, prismatic or pebble-bed form for the reactor fuel. This has implications in the layout of the vessel and its internals particularly from the functional viewpoint of fueling and defueling. The basic concept of the system layout is the same for both designs (both are direct-cycle) as far as the components of the balance of plant (BOP) is concerned. The variation of the Brayton cycle utilized is similar for both plants. However, the GT-MHR design adopts an integral power conversion unit (PCU) in a vessel and a concentric hot/cold duct that connects the reactor system 


\begin{tabular}{|c|lll|}
\hline NEXT GENERATION NUCLEAR PLANT & Identifier: & PLN-2498 \\
METHODS RESEARCH AND & Revision: & 1 & \\
DEVELOPMENT TECHNICAL & Effective Date: & $9 / 25 / 08$ & Page: 132 of 188 \\
PROGRAM PLAN & & & \\
\hline
\end{tabular}

vessel and the PCU system vessel. This leads to a very compact design layout with minimal ducting. On the other hand, the PBMR design adopts distributed PCU components and separated hot and cold ducts. This leads to a larger footprint for the BOP with major lengths of ducting. Thermal stratification in the ducting may be quite different. This is an example of event phenomena differences which the differences in the design may lead to and which will be detailed in the PIRTs.

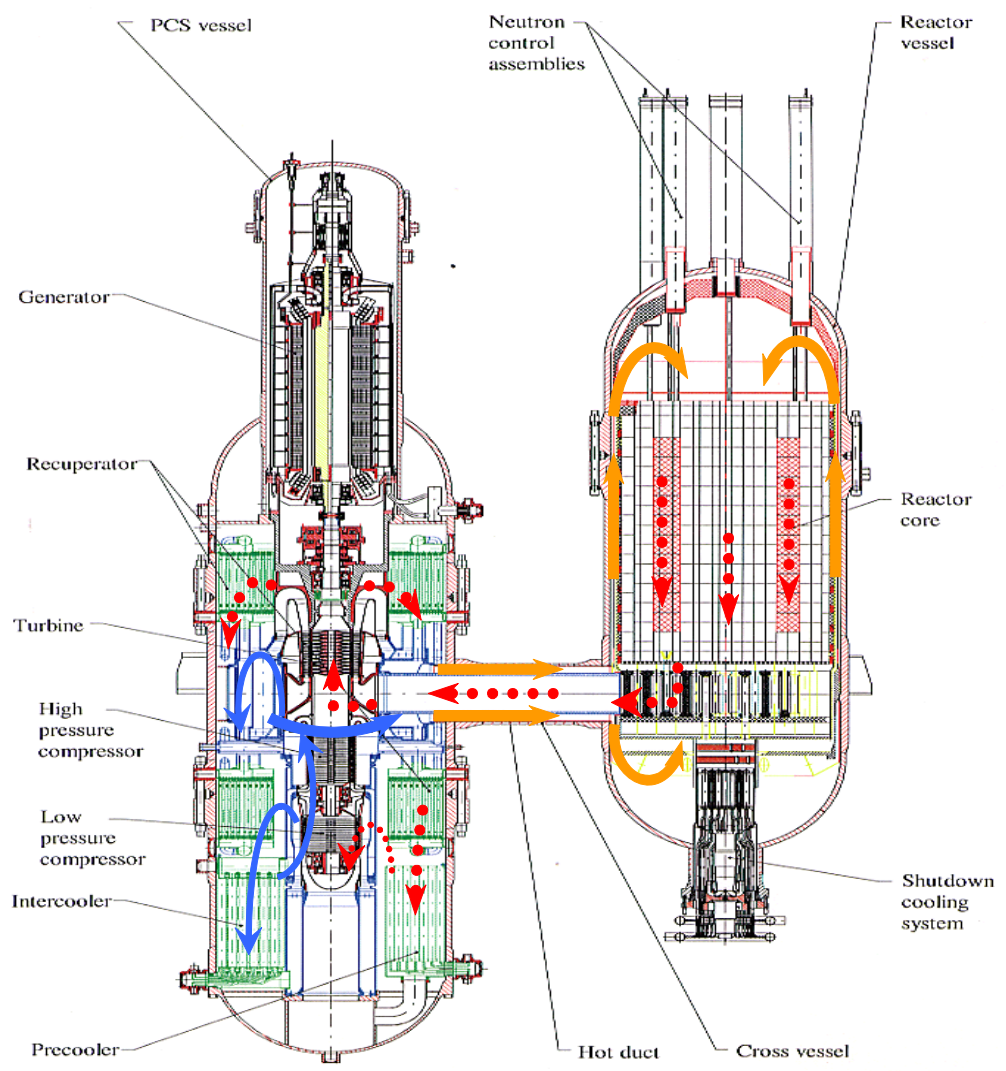

a) System Design Configuration and Helium Flow Path
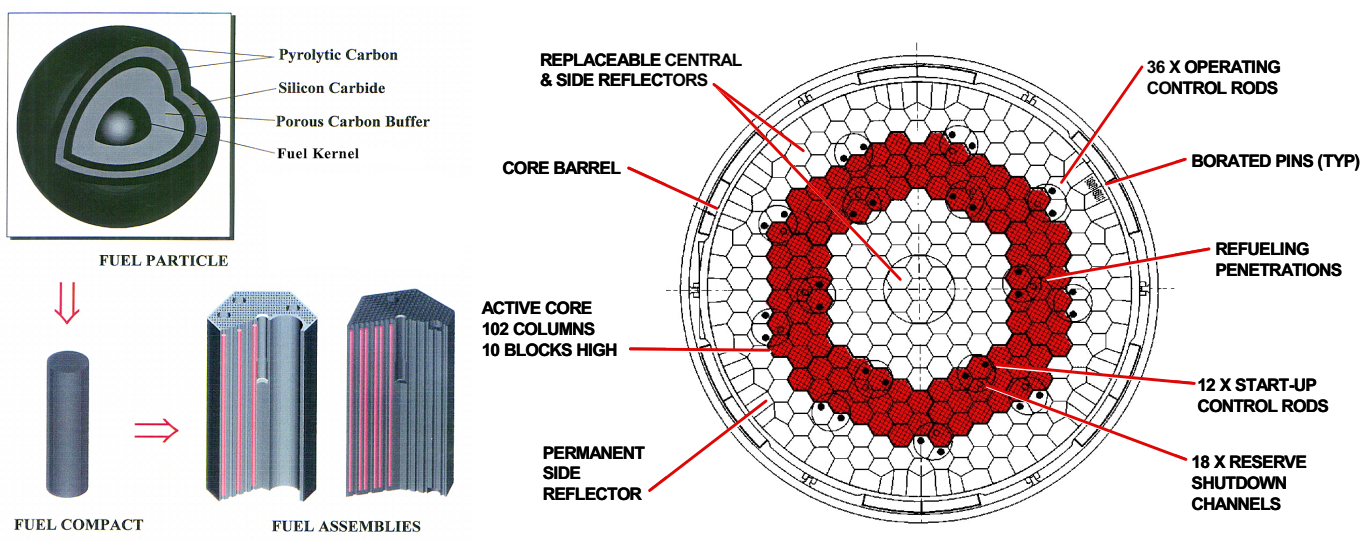

b) Prismatic Core Layout

Figure A-1. GT-MHR Design. 


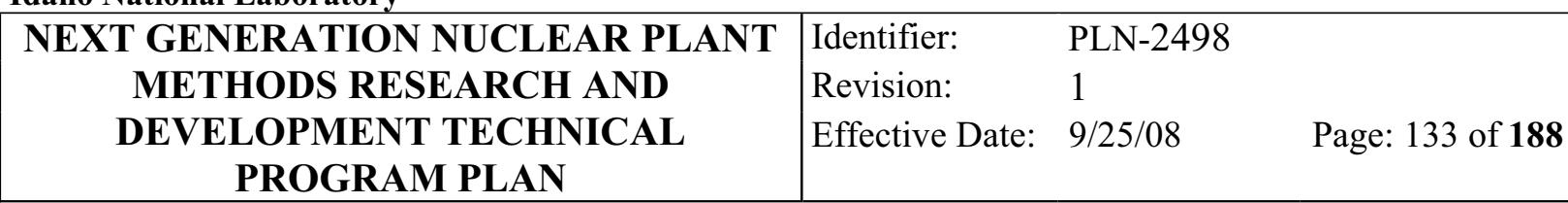

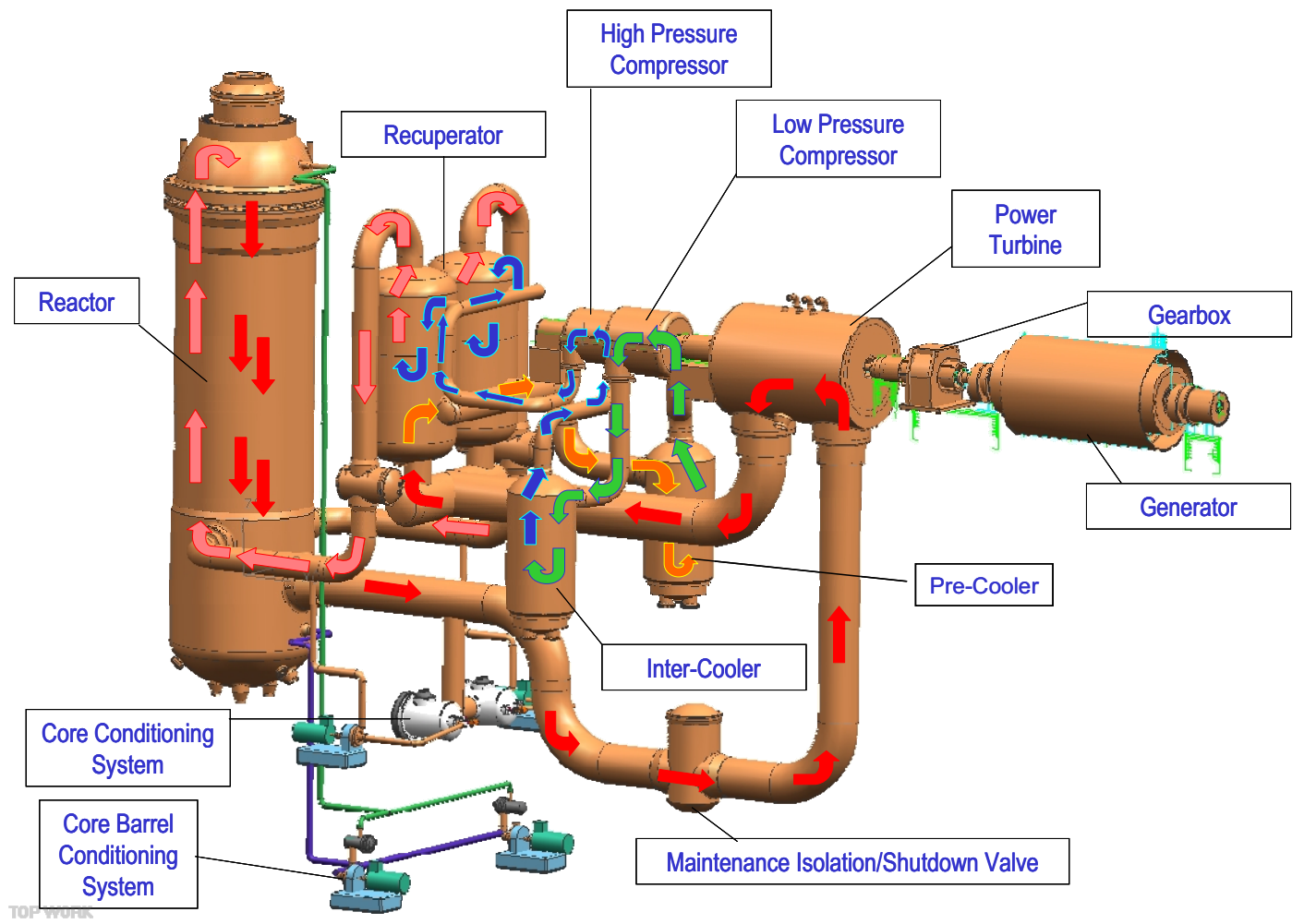

a) System Design Configuration and Helium Flow Path
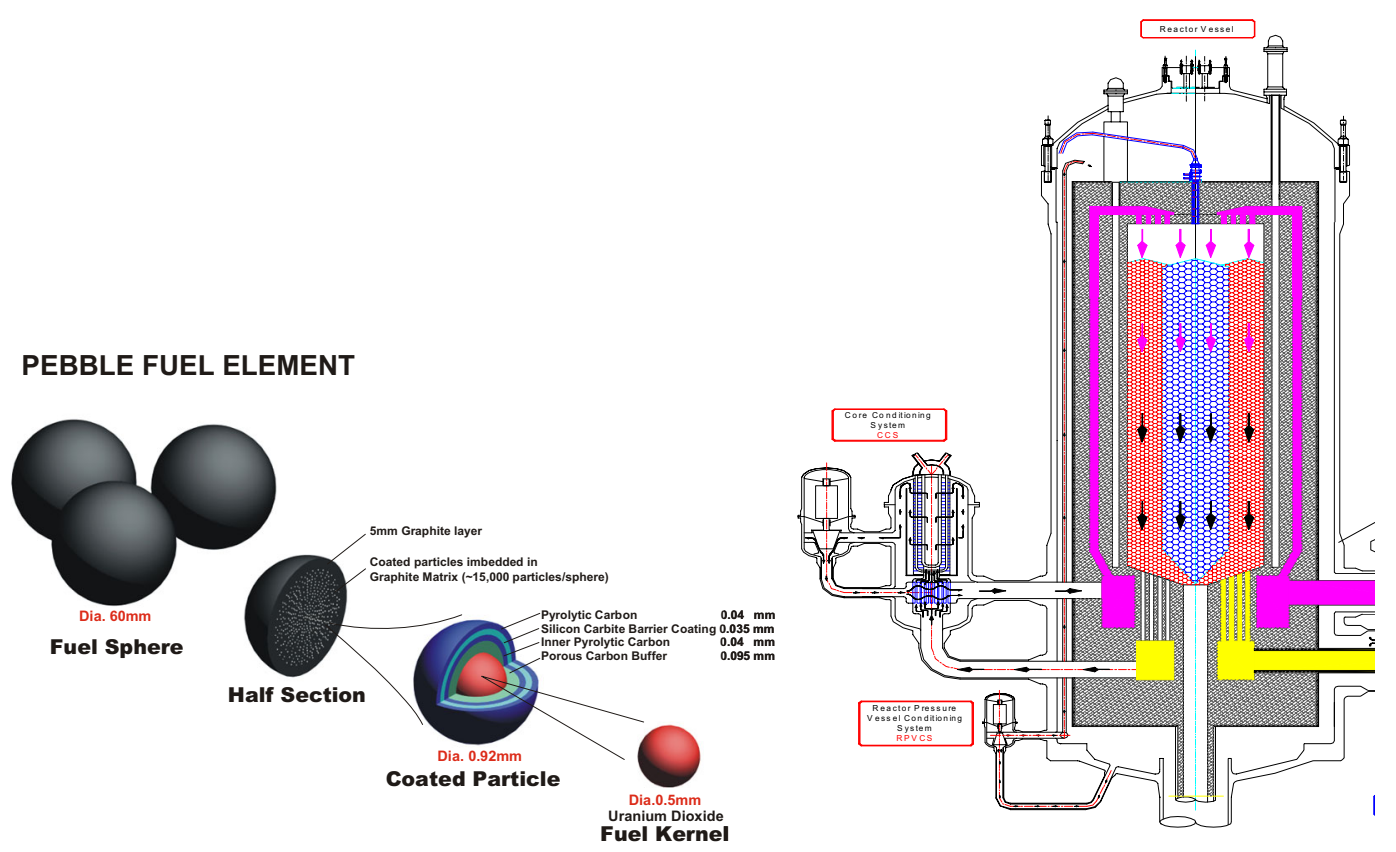

b) Pebble Core and Reactor Vessel Configuration

Figure A-2. PBMR Design. 


\begin{tabular}{|c|lll|}
\hline NEXT GENERATION NUCLEAR PLANT & Identifier: & PLN-2498 & \\
METHODS RESEARCH AND & Revision: & 1 & \\
DEVELOPMENT TECHNICAL & Effective Date: & $9 / 25 / 08$ & Page: 134 of 188 \\
PROGRAM PLAN & & & \\
\hline
\end{tabular}

The prismatic core consists of an inner reflector region surrounded by an annulus of fuel blocks which is in turn surrounded by an annulus of outer reflector elements. The fuel blocks are composed of hexagonal columns of graphite with circular holes that run the full length of the column. The fueled holes contain fuel compact that contains TRISO particles, while the coolant holes align axially to form coolant channels.

The 400 MWth pebble core consists of approximately 450,000 fuel pebbles that are stacked in a graphite reflector structure. The pebbles are continuously refueled during plant operation. Central reflector pebbles have been replaced by central graphite reflector column in the recent design. Characteristic parameters and conditions related to the core are given in Table A-1.

Table A-1. Core Parameters and Full Power Operating Conditions.

\begin{tabular}{|l|l|l|}
\hline \multicolumn{1}{|c|}{ Parameter } & \multicolumn{1}{c|}{ GT-MHR } & \multicolumn{1}{c|}{ PBMR } \\
\hline Reactor Power, Q (Mwt) & 600 & 400 \\
\hline Tin/Tout $\left({ }^{\circ} \mathrm{C}\right)$ & $491 / 850$ & $500 / 900$ \\
\hline Reactor Pressure (bars) & 70 & 90 \\
\hline Power Density (W/cc) & $\sim 5$ & $\sim 6.6$ \\
\hline Reactor Mass Flow rate, W (kg/s) & 320 & 147 \\
\hline Effective Core Height (m) & 7.93 & $\sim 11$ \\
\hline Core Diameter (m) & 2.96 ID/4.83 OD & 2 ID/3.7 OD \\
\hline Number of Fuel Blocks/Pebbles & 1020 & $\sim 450,000$ \\
\hline Design Bypass Flow Fraction (\%) & $10 \sim 15$ & Not applicable \\
\hline
\end{tabular}

\section{A-1.1 Characteristics Common to Both Configurations}

The configurations of the two gas-cooled VHTR candidates are discussed briefly from the perspective of similarities and then the differences are highlighted.

The working fluid is helium, which:

1. Enters the vessel through either a circular cross-section pipe or a pipe annulus near the bottom of the vessel in a direction that is at right angles to the axis of the reactor vessel.

2. Makes a 90-degree turn upward and is distributed, via an upper plenum, into riser channels that lead upward to a upper plenum that is over the core itself.

3. Is directed downward from the upper plenum into the core.

4. Moves from the core into a lower plenum and is directed to a circular cross-section pipe (the hot duct) that is mounted at a right angle to the reactor vessel centerline. As the helium transits the core the gas temperature increases (by $400^{\circ} \mathrm{C}$ to $500^{\circ} \mathrm{C}$ ).

5. Enters the power conversion vessel and is directed to the turbine inlet.

The helium coolant flow distribution in the core is governed by the differential pressure between the upper and lower plena, the friction in the respective flow paths, and the local power generation. The moderator in both reactor configurations is graphite. Moreover, the fuel in both designs consists of TRISO fuel-particles dispersed in a matrix. However, the matrix for the prismatic design is formed into a fuel pin compact while the matrix for the pebble-bed design is formed into a sphere. Both designs rely on 


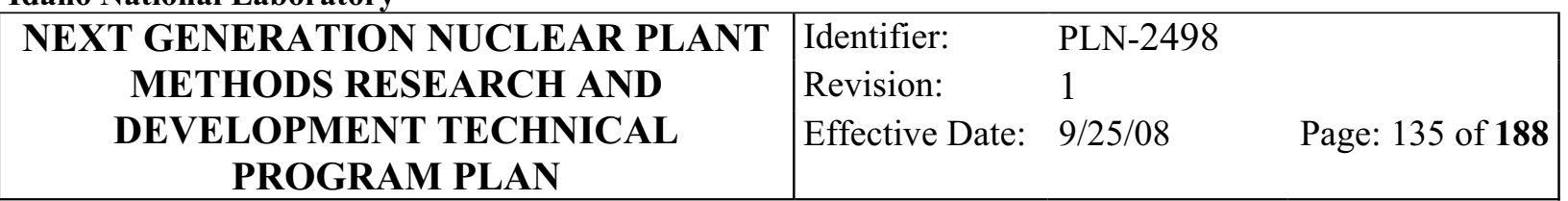

forced flow, provided by blowers, of the helium coolant during operation. Both designs rely on passive cooling during any loss-of-power scenario or loss-of-coolant scenarios. The ultimate heat sink is the environment and all excess heat can be transported to the environment without natural circulation cooling inside the vessel via heat conduction and radiation to the vessel walls. From the vessel walls the heat is transported to the environment via a combination of radiation and natural circulation transport using some form of RCCS. More details on RCCS design are discussed in section A-1.3. Air is present in the confinement such that if the reactor depressurizes due to a leak in a pipe, air will ultimately ingress into the vessel by diffusion.

\section{A-1.2 Fundamental Differences between the Two Configurations}

Core Thermal-Fluids. For the prismatic core, the helium coolant, within the hexagonal blocks, follows well defined one-dimensional (1-D) flow paths described by the coolant channels. However, an undefined quantity of bypass flow, ranging from $\sim 10$ to $\sim 25 \%$ of the total coolant (although the design bypass is 10 to $15 \%$ ), moves between the bocks. The bypass flow varies according to the quality of the block construction, the movement of the graphite as a function of irradiation and temperature, and the core design and stacking procedures. Contact heat transfer between the blocks that plays an important role in transmitting core afterheat during accidents is also affected by them. In contrast, the helium coolant moving through the pebble-bed core follows multi-dimensional flow paths defined by the pebble-void fraction, which varies as a function of core radius, and the individual contact points described by the pebble-bed column. During accidents, radiation and contact heat transfer between pebbles plays an important role in transmitting core afterheat to the reactor vessel wall. The core axial power distribution in the pebble core is more apt to be top-skewed than in the prismatic core due to the online refueling of fresh pebbles from the top.

Reactor Vessel Thermal-Fluids. Even though the underlying design characteristics of both candidates are similar, the detailed designs of the reactor vessel internals are different in the two cases as shown in Figures A-3 and A-4. In the prismatic design, helium flow from the power conversion unit is mixed and redistributed in the upper plenum and flows upward through 6 square riser ducts between the core barrel and the vessel wall. It is collected in the hemispheric upper plenum and then flows downward into the core. In order to prevent overheating at the vessel, a thermal insulation is provided at the inner side of the vessel head. The helium jets discharged from the core are collected and mixed in the lower plenum. The helium then flows out of the vessel to the PCU. In the pebble- bed reactor design, helium flow from the PCU is distributed in a doughnut-like inlet plenum and flows upward through the riser which consists of 36 circular channels inside the outer reflector. The helium passes through the slots at the top of the riser and collects in the cylindrical upper plenum inside the upper graphite structure. It then flows downward to the core. Helium from the core is collected and mixed in the lower plenum and then flows out of the vessel to the PCU.

The pebble-bed core slowly moves downward while the prismatic core is stationary. The cycle time through the core for an individual pebble is approximately 60 to 80 days. The transit distance is $\sim 9.5 \mathrm{~m}$.

The reactor kinetics and burnup characteristics are functions of the fuel and moderator geometry, the fuel enrichment, and the refueling characteristics of the respective designs. Because the pebble-bed core is continuously being replenished as spent pebbles are removed from the system (each pebble is cycled through the core approximately 9 times), the pebble-bed core generally has a wider spectrum of depletion during operation than the prismatic reactor. 


\begin{tabular}{|c|c|c|c|}
\hline NEXT GENERATION NUCLEAR PLANT & Identifier: & PLN-2498 & \\
\hline METHODS RESEARCH AND & Revision: & 1 & \\
\hline $\begin{array}{l}\text { DEVELOPMENT TECHNICAL } \\
\text { PROGRAM PLAN }\end{array}$ & Effective Date: & $9 / 25 / 08$ & Page: 136 of $\mathbf{1 8 8}$ \\
\hline
\end{tabular}

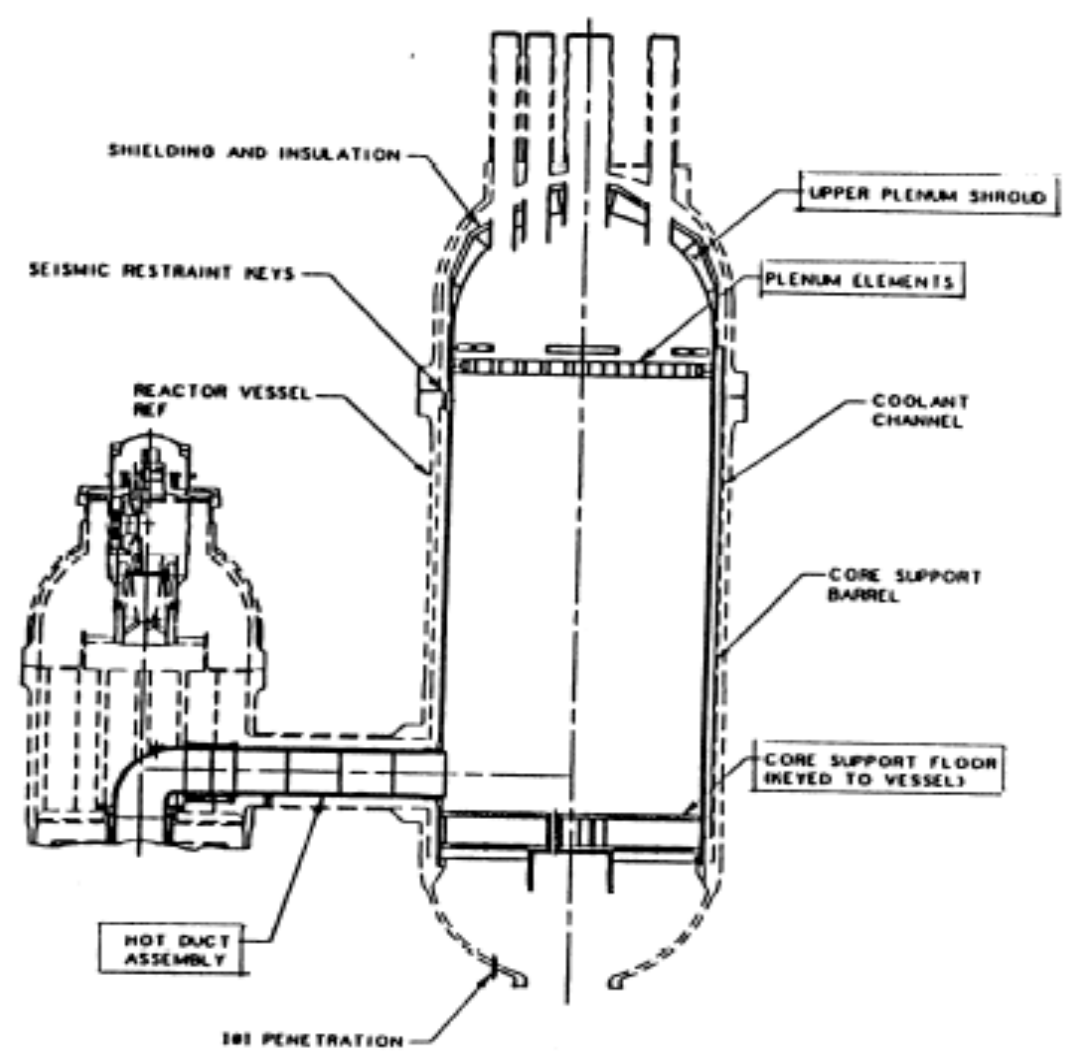

a) Vessel metallic structures

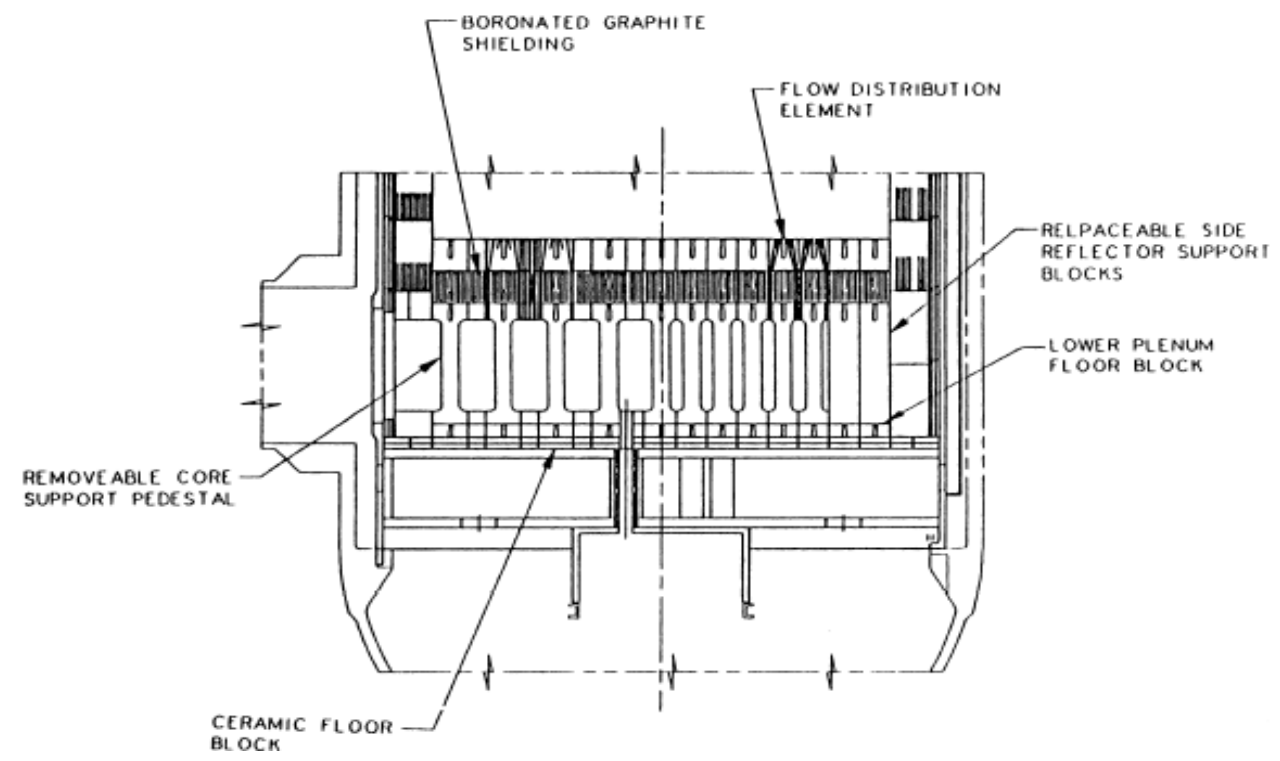

b) Vessel lower plenum

Figure A-3. Prismatic reactor vessel internals. 


\begin{tabular}{|c|lll|}
\hline NEXT GENERATION NUCLEAR PLANT & Identifier: & PLN-2498 \\
METHODS RESEARCH AND & Revision: & 1 & \\
DEVELOPMENT TECHNICAL & Effective Date: & $9 / 25 / 08$ & Page: 137 of 188 \\
PROGRAM PLAN & & & \\
\hline
\end{tabular}

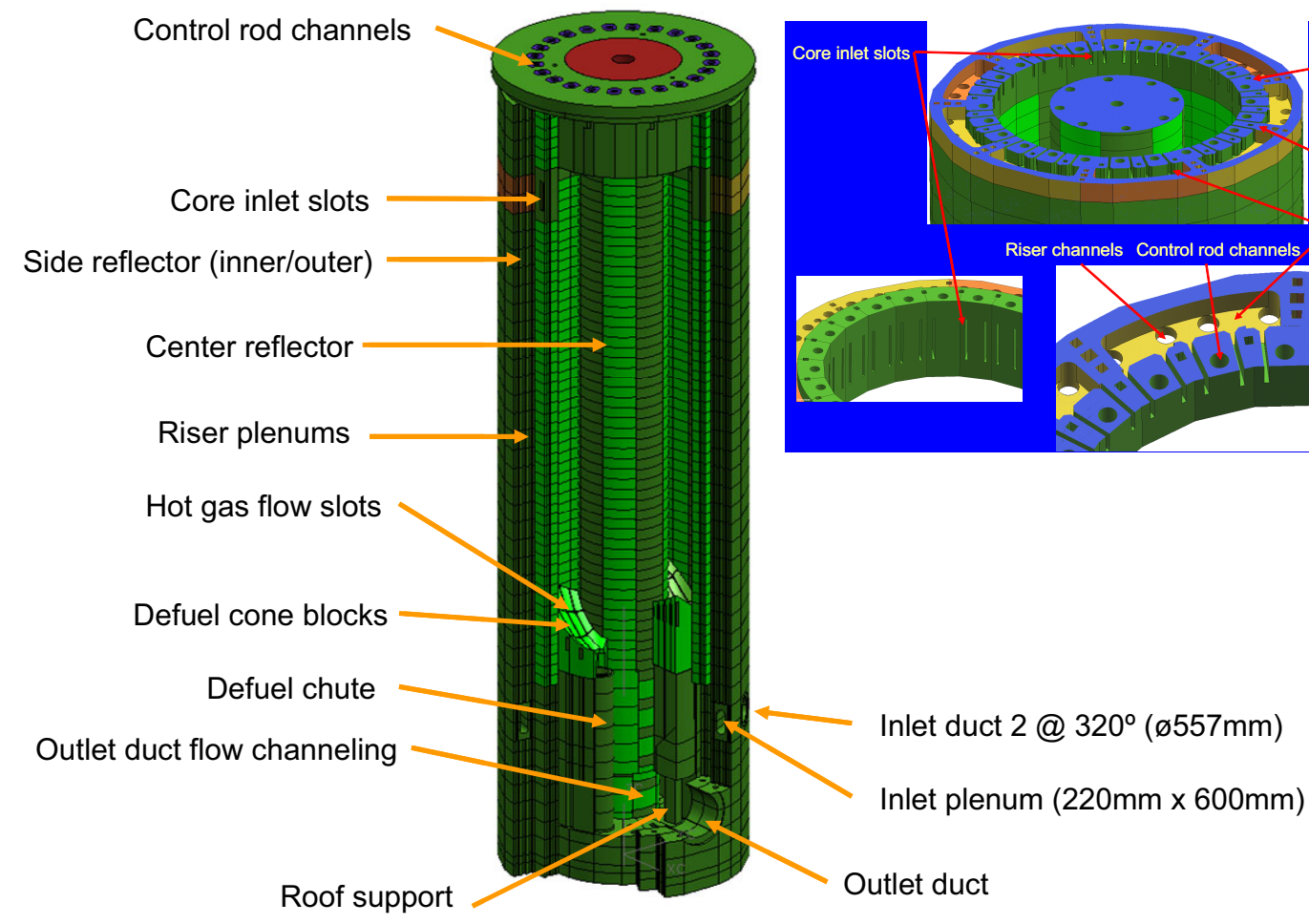

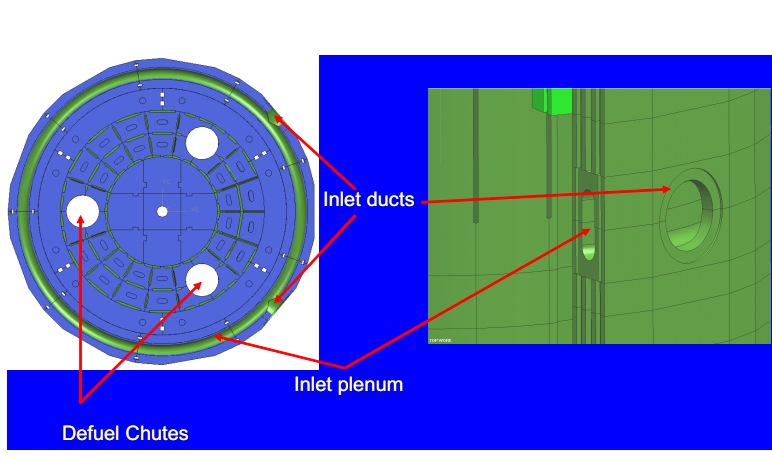

Inlet Plenum

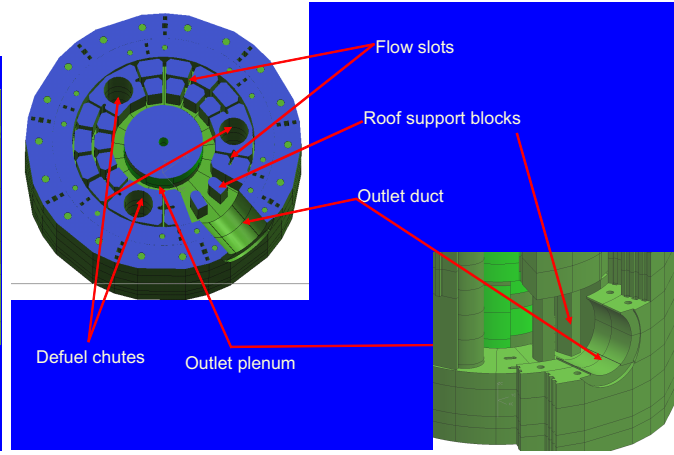

Lower Plenum

Figure A-4. Pebble-bed reactor vessel internals.

\section{A-1.3 Reactor Cavity Cooling System}

There is a major difference in the Reactor Cavity Cooling System (RCCS) design between the two reference designs. The GT-MHR design has an air-cooled RCCS, while the PBMR design has a watercooled RCCS. However, since the details of how the PBMR RCCS functions and its particular design features were not known while this study was initiated and ongoing, an air-cooled RCCS was assumed to be present in both reference designs.

For the assumed air-cooled RCCS design, heat is radiated from the exterior of the reactor vessel wall to a series of heat exchangers that are oriented vertically and arranged in a circle around the exterior of the reactor vessel. Air flowing within these heat exchangers (ducts) transports the heat to the exterior of 


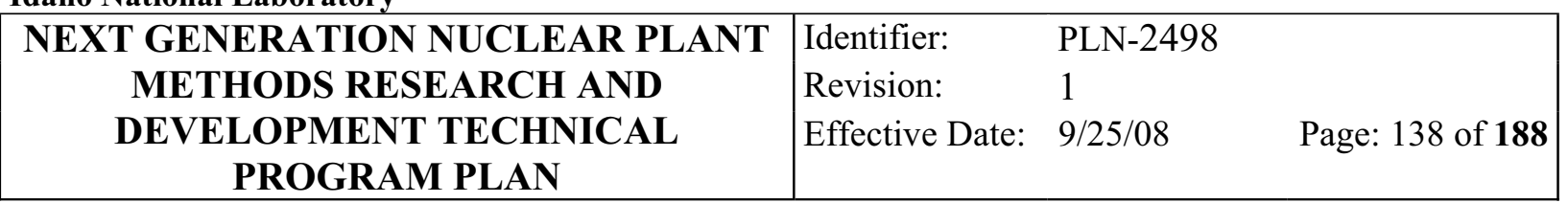

the confinement. The air is ducted in from outside the containment to these heat exchangers and then outside the confinement. The heat exchangers are rectangular ducts with a large aspect ratio and arranged so that one of the short sides faces the reactor vessel. These requirements dictate that the flow exiting the heat exchangers be ducted to chimneys leading to the outside to induce a sufficient natural draft. The walls of the heat exchangers and the ducts that connect to them provide a barrier that separates the coolant flowing through the heat exchangers from the atmosphere inside the reactor/silo confinement. The aircooled RCCS system is designed to be totally passive under all operating condition and has no blowers to power the air flow through the heat exchangers. There are 292 risers, each a 5 by $25.4 \mathrm{~cm}$ rectangular duct. There is a $5-\mathrm{cm}$ gap between adjacent risers and the short sides of each riser face the reactor vessel or the downcomer. The full power thermal-fluid conditions are given in Table A-2 and Figure A-5 is a schematic of the RCCS.

Table A-2. RCCS Duct Dimensions and Operating Conditions.

\begin{tabular}{|l|c|}
\hline \multicolumn{1}{|c|}{ Parameter } & Air-cooled RCCS \\
\hline RCCS Power (MWth) & 3.3 \\
\hline RCCS Air Flowrate $(\mathrm{kg} / \mathrm{s})$ & 14.3 \\
\hline Number of Ducts & 292 \\
\hline Average Duct Air Flowrate $(\mathrm{kg} / \mathrm{s})$ & 0.049 \\
\hline Duct Dimensions $(\mathrm{m})$ & $0.05 \times 0.25$ \\
\hline Hydraulic Diameter $(\mathrm{m})$ & 0.083 \\
\hline Length of Active Core Region $(\mathrm{m})$ & 7.93 \\
\hline
\end{tabular}

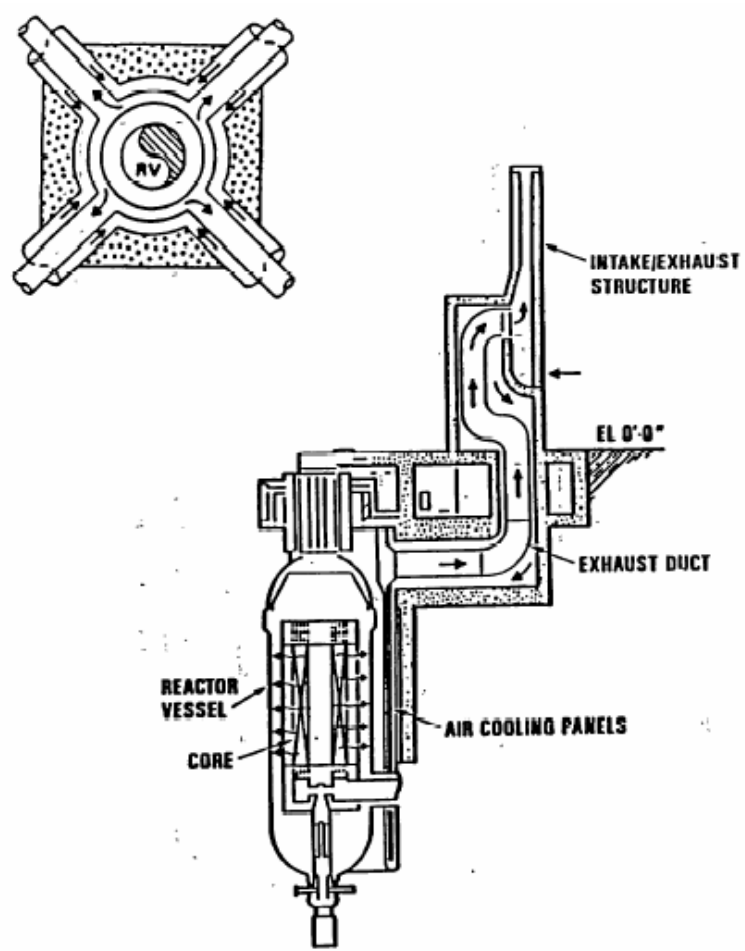

Figure A-5. Schematics of Air-Cooled RCCS. 


\begin{tabular}{|c|lll|}
\hline NEXT GENERATION NUCLEAR PLANT & Identifier: & PLN-2498 \\
METHODS RESEARCH AND & Revision: & 1 & \\
DEVELOPMENT TECHNICAL & Effective Date: & $9 / 25 / 08$ & Page: 139 of 188 \\
PROGRAM PLAN & & & \\
\hline
\end{tabular}

\section{A-1.4 Strengths and Weaknesses of Prismatic Design Relative to the Pebble-Bed Design}

\section{Strengths:}

- Larger fabrication, operating, and licensing experience base in the United States.

- Helium coolant flow paths are well known and relatively controllable due to fixed core design; peak fuel temperature may be more predictable.

- Placement of control rods in fuel region is easier since control rod channels can be drilled into the blocks.

Weaknesses:

- Larger excess reactivity, higher control worth, and relatively high packing fractions required to get desired operating cycle length.

- Must be shut down periodically for refueling and refueling may be relatively complicated.

- Fuel at hot spots remains at same location relatively long time.

- Relatively strong reactivity increase upon significant water ingress.

\section{A-1.5 Strengths and Weaknesses of Pebble-Bed Design Relative to the Prismatic Design}

\section{Strengths:}

- Very little excess reactivity is needed - thus (a) reactivity insertion accident essentially eliminated from consideration and (b) significant reduction in reactivity insertion due to water ingress.

- Very effective fuel utilization.

- Few reactor shutdowns required (no refueling outages).

- Fuel enrichment is lower and thus the fuel costs are probably lower.

- Peak fuel temperatures will probably be lower.

Weaknesses:

- It is likely to be more difficult to calculate flow and temperature variations for the pebble-bed than the prismatic configuration.

- More pebble withdraw tubes are needed for annular core than for a solid core; bridging and stuck pebbles are a possibility.

- Larger pressure drops across core (for $10 \mathrm{~m}$ high core). However, cross-flow design may eliminate this issue.

- Production of dust. AVR generated approximately $3 \mathrm{~kg} / \mathrm{year}$ from control rod insertions, rubbing of fuel pebbles, and drag of fuel pebbles on the vessel shroud. 


\begin{tabular}{|c|lll|}
\hline NEXT GENERATION NUCLEAR PLANT & Identifier: & PLN-2498 \\
METHODS RESEARCH AND & Revision: & 1 & \\
DEVELOPMENT TECHNICAL & Effective Date: & $9 / 25 / 08$ & Page: 140 of 188 \\
PROGRAM PLAN & & & \\
\hline
\end{tabular}

- Potentially may be more difficult to license because the moving fuel increases the uncertainty of the fuel performance in terms of temperature, power history, time in the core, and exact calculations of hot spots and blockages that may occur.

Because of the similarities between the prismatic and pebble-bed designs, the same thermal-fluid tools can probably be used to perform the required analyses for both systems. However, there will be validation requirements that are specific to each of these designs. Also, some of the R\&D will focus on the respective strengths and weaknesses identified above in an attempt to clarify the magnitude of the differences between the designs.

\section{A-1.6 Operational Conditions}

The neutronic and thermal-fluid behavior at rated operational conditions must be calculated to enable design studies to be conducted by INL and/or to perform audit calculations on designs submitted by potential VHTR commercial design teams. Such calculations are centered in two areas:

- Calculations of the neutronic behavior of the proposed design include

- Block loading studies for the prismatic design and pebble loading for the pebble-bed design

- Block and/or pebble-bed k $\infty$ versus packing fraction

- Reactivity effects caused by the working fluid

- Model k-effective as function of temperature and core geometry

- Core k-effective versus core enrichment

- Neutron flux, fluence, displacements per atom, and spectra

- Effect of water ingress on reactivity

- Fuel block depletion and pebble depletion

- Core depletion

- Temperature coefficients of reactivity

- Fuel rod power peaking and/or pebble power peaking.

- Control rod worths and impact.

- Calculations of the thermal-fluid behavior of system to determine

- Helium gas exit temperature from hottest channel

- Variation in temperature between hottest and coldest jets into lower plenum

- Degree of mixing that occurs in the lower plenum and the translation of the temperature distribution to the turbine inlet

- Losses to the environment via the reactor containment cooling system, since this system is always operational

- Peak temperatures in the channels in the prismatic design and among the pebbles in the pebblebed design; peak temperatures in the structural members of both systems

- Evaluation of thermal stresses in fuel and system structural members.

\section{A-1.7 Transient Conditions}

The neutronic and thermal-fluid behavior during the most severe transient scenarios must be calculated to enable design studies to be conducted by the NGNP Project and/or to perform audit 


\begin{tabular}{|c|lll|}
\hline NEXT GENERATION NUCLEAR PLANT & Identifier: & PLN-2498 \\
METHODS RESEARCH AND & Revision: & 1 & \\
DEVELOPMENT TECHNICAL & Effective Date: & $9 / 25 / 08$ & Page: 141 of 188 \\
PROGRAM PLAN & & & \\
\hline
\end{tabular}

calculations on designs submitted by potential VHTR commercial design teams. Such calculations are centered in two areas:

- Calculations of the neutronic behavior of the proposed design include consideration of

- Decay heat

- Local and global power imbalances resulting from inadvertent rod ejection (Morris, Petti, Powers, and Boyack, 2004)

- Water ingress.

- Impact of reactivity driven events, e.g., control rod ejections, failures, and seismic repacking of the pebble-bed.

- Calculations of the thermal fluid behavior of the proposed design include consideration of

- Heat transfer to environment during conduction cooldown scenarios (both pressurized and depressurized)

- Peak temperatures in structural members

- Peak temperatures in fuel

- Mixing in upper and lower plenum during PCC

- Natural convection and radiation heat transfer in RCCS.

\section{A-2. SELECTION OF SCENARIOS AND PRIMARY SAFETY CRITERIA}

The full spectrum of scenarios of importance is not yet defined for the VHTR, since it is intimately linked to the presently undefined VHTR design. However, on the basis of the work done to license the Fort Saint Vrain reactor, it is known that the following scenarios, at least, must be analyzed, as indicated in the Fort Saint Vrain FSAR:

1. Anticipated operational occurrences:
a. Main loop transient with forced core cooling
b. Loss of main and shutdown cooling loops
c. Accidental withdrawal of a group of control rods followed by reactor shutdown
d. Small break LOCA $(\sim 1$ in2 area break).

2. Design basis accidents (assuming that only "safety-related" systems can be used for recovery):
a. Loss of heat transport system and shutdown cooling system (similar to scenario $1 \mathrm{~b}$ above)
b. Loss of heat transport system without control rod trip
c. Accidental withdrawal of a group of control rods followed by reactor shutdown
d. Unintentional control rod withdrawal together with failure of heat transport systems and shutdown cooling system
e. Earthquake-initiated trip of heat transport system 


\begin{tabular}{|c|lll|}
\hline NEXT GENERATION NUCLEAR PLANT & Identifier: & PLN-2498 \\
METHODS RESEARCH AND & Revision: & 1 & \\
DEVELOPMENT TECHNICAL & Effective Date: & $9 / 25 / 08$ & Page: 142 of 188 \\
PROGRAM PLAN & & & \\
\hline
\end{tabular}

f. LOCA event in conjunction with water ingress from failed shutdown cooling system

g. Large break LOCA

h. Small break LOCA

On the basis of the experience of gas-cooled reactor designers and experimentalists (Ball, 2003; Krüger, et al, 1991), scenarios 2a and $2 \mathrm{~g}$ (hereafter referred to as the PCC and the DCC, respectively) are considered the most demanding and most likely to lead to maximum vessel wall and fuel temperatures. Hence, these two PCC and DCC events were selected as the nucleus events. In addition, in order to include the phenomena that occur during normal and operational transients, the load change from full power to a reduced power condition was selected as the third scenario.

The primary safety criteria are defined to be the fuel and vessel temperatures for the PCC and DCC scenarios and the local hot spot for the load change scenario. Table A-3 summarizes the selected nucleus set of events and corresponding primary safety criteria.

Table A-3. Selected Nucleus Set of Events and Primary Safety Criteria.

\begin{tabular}{|l|l|l|}
\hline \multicolumn{1}{|c|}{ ID } & \multicolumn{1}{|c|}{ Event } & \multicolumn{1}{|c|}{ Safety Criteria } \\
\hline PCC & $\begin{array}{l}\text { Loss of Primary Flow and shutdown cooling systems } \\
\text { (PCC Event) }\end{array}$ & $\begin{array}{l}\text { Fuel and Vessel } \\
\text { Temperature }\end{array}$ \\
\hline DCC & $\begin{array}{l}\text { LOCA with air-ingress } \\
\text { (DCC Event) }\end{array}$ & $\begin{array}{l}\text { Fuel and Vessel } \\
\text { Temperature }\end{array}$ \\
\hline LC & $\begin{array}{l}\text { Load Changes } \\
\text { (including steady-state operation as Phase 1) }\end{array}$ & Local Hot Spot \\
\hline
\end{tabular}

\section{A-3. IDENTIFICATION OF ASSOCIATED COMPONENTS}

The phenomena of importance are specific to each system region for any given scenario. For example, in the prismatic variant of the VHTR, the core is composed of hexagonal cross-section blocks with over a hundred cooling channels in each block. From a thermal-fluid perspective one of the phenomena of importance for both steady-state conditions and the pressurized conduction cool down scenario is the heat transfer to the coolant in each of the cylindrical cooling channels and consequently the coolant exit temperature. However, in the reactor vessel outlet plenum the phenomena of importance, at least at steady-state conditions, is the mixing of the gas and the probability of having excessively hot gases impinge on the plenum walls. Consequently, the system must be divided into regions prior to identifying the major phenomena of importance for any given scenario. Table A-4 lists the regions in either a prismatic or pebble-bed system.

The reactor vessel is composed of an inlet plenum, the risers, the upper plenum and components, reflectors (including the bypass), the core and fuel (including an additional bypass component), and the lower plenum and components. The inlet plenum is located just downstream of the vessel inlet helium gas duct and is the volume that feeds the helium to risers. Helium flows through the risers into the upper plenum prior to continuing its travel through the core cooling channels and then to the lower plenum. From the lower plenum the working fluid moves into the hot duct and from thence into the power conversion system (only a direct cycle system will be considered together with the presence of an IHX). 


\begin{tabular}{|c|lll|}
\hline NEXT GENERATION NUCLEAR PLANT & Identifier: & PLN-2498 \\
METHODS RESEARCH AND & Revision: & 1 & \\
DEVELOPMENT TECHNICAL & Effective Date: & $9 / 25 / 08$ & Page: 144 of 188 \\
PROGRAM PLAN & & & \\
\hline
\end{tabular}

Once the system coast down is completed, the system is left in a state where the controlling boundary conditions that govern the peak system temperatures are the system power level, the heat transfer from the fuel to the core, to the vessel, and then to the environment via the RCCS. The core heat-up slows down by the natural circulation cooling developed inside the core and the increase of heat removal by conduction and radiation cooling to the RCCS. Eventually, the core cools down when the heat removal by the conduction and radiation cooling becomes larger than the core afterheat and the condition approaches a safe shutdown state. The temperatures of concern are the fuel temperature and that of the vessel structural components.

Consequently, there are two phases envisioned: (1) the coastdown and (2) the heat-up and passive cooling phases. Table A-5 summarizes the PCC scenario and event phases identified for the PIRT process.

Table A-5. PCC Scenario and Accident Phases.

\begin{tabular}{|l|l|l|}
\hline Phases & \multicolumn{1}{|c|}{ Phase ID } & \multicolumn{1}{c|}{ Event Scenarios and Major Processes } \\
\hline 1 & Coastdown & $\begin{array}{l}\text { Event initiated by loss of offsite power and failure of shutdown cooling } \\
\text { system to start } \\
\text { Reactor trips } \\
\text { Coast down of primary flow results in "rapid" increase of fuel } \\
\text { temperature, while reactor vessel temperature decreases with the loss of } \\
\text { forced flow }\end{array}$ \\
\hline 2 & $\begin{array}{l}\text { Heat-up and } \\
\text { Passive Cooling }\end{array}$ & $\begin{array}{l}\text { Core heat-up slows down by the natural circulation cooling inside the } \\
\text { core and the increase of heat removal by conduction and radiation } \\
\text { cooling to RCCS } \\
\text { Core cools down when the heat removal by conduction and radiation } \\
\text { cooling to RCCS becomes larger than core afterheat } \\
\text { Core reaches a safe shutdown state }\end{array}$ \\
\hline
\end{tabular}

\section{A-4.2 Depressurized Conduction Cooling (DCC) Event}

The DCC scenario begins from a $100 \%$ power condition and is initiated by a double-ended guillotine break of both the cold and hot ducts. After the break, the reactor system blows down quickly. Reactor trips immediately to decrease the core power down to decay heat level. Nevertheless, core heats up rapidly by the decrease in heat removal by the loss of forced convection and the depressurization. Due to the loss of coolant, the power conversion unit is disconnected and does not significantly affect the progression of the transient. Helium discharge into the reactor cavity stops when the pressures of the reactor system and cavity equalize. Helium discharge from the reactor system purges part of the air in the cavity into the compartments in the confinement. Thus, the gas species in the cavity is redistributed. During blowdown, graphite dust accumulated in the reactor system is also transported into the confinement and eventually released to environment through confinement relief valve. Filtered venting can reduce the release of dust to the environment.

Once the system blowdown is complete, the system is left in a state where the controlling boundary conditions that govern the peak system temperatures are the system power level, the heat transfer from the fuel to the core, to the vessel, and then to the environment via the RCCS. The fuel temperature continues to increase and experiences the first peak when the core afterheat is balanced by the conduction and radiation cooling to the RCCS. During this phase, air remaining in the reactor cavity continuously enters 


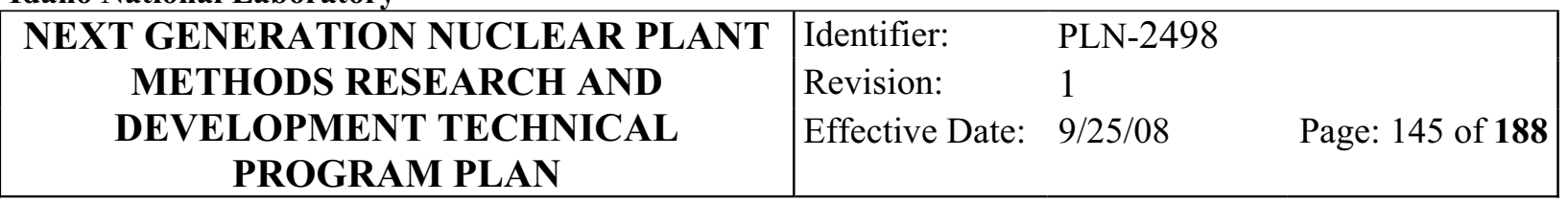

into the reactor vessel by molecular diffusion. Because the diffusion process is very slow, the graphite chemical reaction rate with oxygen is very slow. Core heat-up slows down by the increase of heat removal to the RCCS. There exists a very weak natural circulation inside the core even at low pressure, but, it is not sufficient to redistribute the core temperature profile. With the increase of RCCS heat removal, the fuel temperature turns down after the first peak. Due to continuous air inflow, the density of the gas mixture in the core gradually increases. The temperatures of concern are the fuel temperature and that of the vessel structural components.

Once the bulk of the air diffuses into the reactor vessel, onset of bulk natural circulation is initiated by the density differences in the air mixture. Then, the extensive graphite oxidation occurs and generates a large amount of heat, which results in the second peak in the core temperature. Graphite oxidation stops when the air in the reactor vessel is depleted, and then, the fuel temperature starts to decrease. The core afterheat is continually removed by the bulk natural circulation cooling and by the conduction and radiation cooling to the RCCS. Eventually, the core cools down when the heat removal by the conduction and radiation cooling becomes larger than the core afterheat and reaches a safe shutdown state. The temperatures of concern are the fuel temperature and that of the vessel structural components.

Consequently, there are three phases envisioned: (1) the blowdown, (2) the molecular diffusion and (3) the air mixture natural convection phase. Table A-6 summarizes the DCC scenario and event phases identified.

Table A-6. DCC Scenario and Accident Phases.

\begin{tabular}{|c|c|c|}
\hline Phases & Phase ID & Event Scenarios and Major Processes \\
\hline 1 & Blowdown & $\begin{array}{l}\text { Event initiated by a double ended break of both cold and hot ducts } \\
\text { System depressurizes and reactor trips immediately } \\
\text { He discharge into reactor cavity stops when the pressures of primary } \\
\text { system and cavity equalize. He discharge from primary system purges part } \\
\text { of air in cavity, thus, gas species in cavity are redistributed } \\
\text { "Rapid" Heat-up of core occurs by the loss of forced convection } \\
\text { Graphite dust from core is transported to cavity then to confinement } \\
\text { The confinement relief valves lift and effluent is released to environment. } \\
\text { Filters minimize distribution of dust to environment. }\end{array}$ \\
\hline 2 & $\begin{array}{l}\text { Molecular } \\
\text { Diffusion }\end{array}$ & $\begin{array}{l}\text { Air remaining in reactor cavity enters into reactor vessel by molecular } \\
\text { diffusion. Thus, graphite chemical reaction rate is very slow } \\
\text { Very weak natural circulation occurs inside the core } \\
\text { First peak of fuel temperature occurs, then, it starts to decrease when the } \\
\text { heat removal by conduction and radiation cooling to RCCS overrides core } \\
\text { afterheat }\end{array}$ \\
\hline 3 & $\begin{array}{l}\text { Natural } \\
\text { convection }\end{array}$ & $\begin{array}{l}\text { Large amount of air ingresses into reactor vessel at onset of natural } \\
\text { circulation due to density difference of gas mixture } \\
\text { Second peak of fuel temperature occurs with graphite oxidation } \\
\text { Graphite oxidation stops when air is depleted. Then, fuel temperature } \\
\text { starts to decrease } \\
\text { Core cools down when the heat removal by conduction and radiation } \\
\text { cooling to RCCS becomes larger than core afterheat and the oxidation. } \\
\text { Core reaches a safe shutdown state }\end{array}$ \\
\hline
\end{tabular}




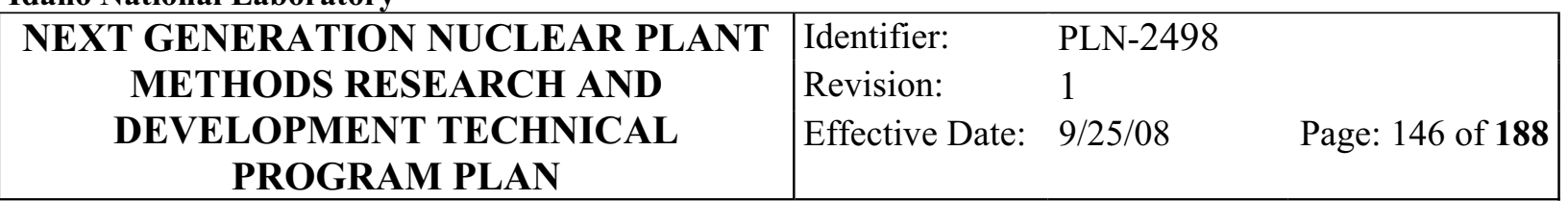

\section{A-4.3 Load Change Event}

The load changes event is initiated by the flow reduction or increase dictated by the Helium inventory control or turbine bypass flow control during normal operation. During the event, reactor trip does not occur and the power conversion unit remains online and connected to the reactor system. The core power varies in accordance with the reactivity feedbacks, principally the fuel Doppler coefficient. Decrease or increase in primary flow results in fuel temperature increase or decrease, which further results in core power decrease or increase through the fuel Doppler reactivity feedback. The major phenomena in this event would include the neutronic transients of the core as well as the response of power conversion unit components such as the recuperator, coolers, turbo-machinery, etc. A compressor surge, with an accompanying pressure pulse, may occur. Also, from the thermal-fluid (gas dynamics) perspective, especially during the flow reduction and recovery phases, the flow asymmetry in the plena (both upper and lower) might be even augmented. After the flow reaches at certain level, the core power also reaches a certain steady level given by the reactivity feedback balances and/or control rods movement.

Consequently, there are two phases envisioned: (1) the flow transient and (2) the steady phase. Table A-7 summarizes the load change scenario and event phases identified.

Table A-7. Load Changes Scenario and Accident Phases.

\begin{tabular}{|l|l|l|}
\hline Phases & \multicolumn{1}{|c|}{ Phase ID } & \multicolumn{1}{c|}{ Event Scenarios and Major Processes } \\
\hline 1 & $\begin{array}{l}\text { Flow } \\
\text { Transient }\end{array}$ & $\begin{array}{l}\text { Event initiated by flow reduction or increase by helium inventory or } \\
\text { turbine bypass flow control } \\
\text { Decrease in flow results in the increase of fuel temperature. Then, core } \\
\text { power decreases by doppler reactivity feedback, while flow increase } \\
\text { results in power increase } \\
\text { A compressor surge, with an accompanying pressure pulse, occurs. }\end{array}$ \\
\hline 2 & Steady & $\begin{array}{l}\text { Core power reaches a certain steady level balanced by reactivity } \\
\text { feedback and/or control rod movement } \\
\text { Steady power operation is included in this phase }\end{array}$ \\
\hline
\end{tabular}

\section{A-5. IDENTIFICATION OF MAJOR PHENOMENA BY COMPONENTS}

The important phenomena that are expected to occur in each component during the progress of events are identified in this section - together with their rankings. The following tables summarize the phenomena by components identified for each time phase of three event scenarios.

The phenomena rankings were performed a PIRT Panel formed by a collaborative effort between the ANL, the Korea Atomic Energy Research Institute, and INL. The US PIRT panel consists of 4 experts from ANL and 3 experts from INL. The Korean PIRT panel consists of 3 experts from universities, 1 from industry, 1 from regulation and 10 from research organization. Ranking has been carried out in accordance with independent ranking method of each organization.

The final rankings are given in the following tables for each region of the system. The left-hand column lists the major phenomena for the component and along the top row are the tree events, each divided into transient phases. A phenomenon was ranked in two levels, either high (H), medium (M). 
Identifier: $\quad$ PLN-2498

Revision: $\quad 1$

Effective Date: 9/25/08

\section{A-5.1 Reactor Vessel (RV) Components}

Table A-8. Core and Reflector (including Bypass) Phenomena Rankings.

\begin{tabular}{|l|c|c|c|c|c|c|c|}
\hline \multirow{2}{*}{\multicolumn{1}{c|}{ Phenomena }} & \multicolumn{2}{|c|}{ PCC } & \multicolumn{3}{c|}{ DCC } & \multicolumn{2}{c|}{ LC } \\
\cline { 2 - 8 } & 1 & 2 & 1 & 2 & 3 & 1 & 2 \\
\hline a. Flow distribution & $\mathrm{H}$ & $\mathrm{H}$ & $\mathrm{H}$ & $\mathrm{H}$ & $\mathrm{H}$ & $\mathrm{H}$ & $\mathrm{H}$ \\
\hline b. Heat transfer (forced convection) & $\mathrm{H}$ & & $\mathrm{H}$ & & & $\mathrm{H}$ & $\mathrm{H}$ \\
\hline c. Heat transfer (mixed and free convection) & & $\mathrm{H}$ & & $\mathrm{M}$ & $\mathrm{H}$ & & \\
\hline d. Pressure drop (forced convection) & $\mathrm{M}$ & & $\mathrm{H}$ & & & $\mathrm{H}$ & $\mathrm{H}$ \\
\hline e. Pressure drop (mixed and free convection) & & $\mathrm{H}$ & & $\mathrm{M}$ & $\mathrm{H}$ & & \\
\hline f. Initial stored energy & $\mathrm{H}$ & & $\mathrm{H}$ & & & & \\
\hline g. Power distribution & $\mathrm{H}$ & $\mathrm{H}$ & $\mathrm{H}$ & $\mathrm{H}$ & $\mathrm{H}$ & $\mathrm{H}$ & $\mathrm{H}$ \\
\hline h. Decay heat(including power distribution) & $\mathrm{H}$ & $\mathrm{H}$ & $\mathrm{H}$ & $\mathrm{H}$ & $\mathrm{H}$ & & \\
\hline i. Reactivity feedback & & & & & & $\mathrm{H}$ & $\mathrm{M}$ \\
\hline j. Fuel/reflector conductivity & $\mathrm{M}$ & $\mathrm{H}$ & $\mathrm{H}$ & $\mathrm{H}$ & $\mathrm{H}$ & $\mathrm{M}$ & $\mathrm{M}$ \\
\hline k. Fuel/reflector specific heat & $\mathrm{M}$ & $\mathrm{H}$ & $\mathrm{H}$ & $\mathrm{H}$ & $\mathrm{H}$ & $\mathrm{M}$ & $\mathrm{M}$ \\
\hline 1. Multi-D heat conduction including contact & $\mathrm{H}$ & $\mathrm{H}$ & $\mathrm{H}$ & $\mathrm{H}$ & $\mathrm{H}$ & $\mathrm{M}$ & $\mathrm{M}$ \\
\hline m. Gas conduction (including gaps) & & $\mathrm{M}$ & & $\mathrm{M}$ & $\mathrm{M}$ & & \\
\hline n. Radiation heat transfer & & $\mathrm{H}$ & & $\mathrm{H}$ & $\mathrm{H}$ & & \\
\hline o. Graphite oxidation & & & & $\mathrm{M}$ & $\mathrm{H}$ & & \\
\hline p. Bulk CO reaction & & & & $\mathrm{M}$ & $\mathrm{H}$ & & \\
\hline q. Molecular diffusion & & & & $\mathrm{M}$ & & & \\
\hline r. Fluid properties (gas mixture) & & & $\mathrm{H}$ & $\mathrm{H}$ & & \\
\hline s. Core material distribution (configuration) & $\mathrm{H}$ & $\mathrm{H}$ & $\mathrm{H}$ & $\mathrm{H}$ & $\mathrm{H}$ & & \\
\hline
\end{tabular}

Table A-9. Inlet Plenum Phenomena Rankings.

\begin{tabular}{|c|c|c|c|c|c|c|c|}
\hline \multirow[b]{2}{*}{ Phenomena } & \multicolumn{2}{|c|}{ PCC } & \multicolumn{3}{|c|}{ DCC } & \multicolumn{2}{|c|}{$\mathrm{LC}$} \\
\hline & 1 & 2 & 1 & 2 & 3 & 1 & 2 \\
\hline a. Flow distribution & $\mathrm{H}$ & & $\mathrm{H}$ & & & $\mathrm{H}$ & $\mathrm{H}$ \\
\hline b. Heat transfer (forced convection) & $\mathrm{M}$ & & M & & & & \\
\hline c. Heat transfer (mixed and free convection) & & & & & $\mathrm{M}$ & & \\
\hline d. Pressure drop (forced convection) & $\mathrm{M}$ & & M & & & $\mathrm{H}$ & $\mathrm{H}$ \\
\hline e. Pressure drop (mixed and free convection) & & & & & M & & \\
\hline f. Bulk CO reaction & & & & M & & & \\
\hline g. Molecular diffusion & & & & M & & & \\
\hline h. Thermal mixing and stratification & & & & M & & & \\
\hline i. Graphite oxidation (PBR) & & & & M & & & \\
\hline j. Fluid properties (gas mixture) & & & & M & & & \\
\hline
\end{tabular}




\begin{tabular}{|c|c|c|c|}
\hline $\begin{array}{l}\text { EXT GENERATION NUCLEAR PLANT } \\
\text { METHODS RESEARCH AND }\end{array}$ & $\begin{array}{l}\text { Identifier: } \\
\text { Revision: }\end{array}$ & PLN-2498 & \\
\hline $\begin{array}{l}\text { DEVELOPMENT TECHNICAL } \\
\text { PROGRAM PLAN }\end{array}$ & Effective Date: & $9 / 25 / 08$ & Page: 148 of $\mathbf{1 8 8}$ \\
\hline
\end{tabular}

Table A-10. Riser Phenomena Rankings.

\begin{tabular}{|l|c|c|c|c|c|c|c|}
\hline \multirow{2}{*}{\multicolumn{1}{c|}{ Phenomena }} & \multicolumn{2}{c|}{ PCC } & \multicolumn{3}{c|}{ DCC } & \multicolumn{2}{c|}{ LC } \\
\cline { 2 - 8 } & 1 & 2 & 1 & 2 & 3 & 1 & 2 \\
\hline a. Flow distribution & $\mathrm{H}$ & $\mathrm{M}$ & $\mathrm{M}$ & & & & \\
\hline b. Heat transfer (forced convection) & $\mathrm{M}$ & & $\mathrm{M}$ & & & & \\
\hline c. Heat transfer (mixed and free convection) & & $\mathrm{M}$ & & & $\mathrm{M}$ & & \\
\hline d. Pressure drop (forced convection) & $\mathrm{H}$ & & $\mathrm{H}$ & & & $\mathrm{H}$ & $\mathrm{H}$ \\
\hline e. Pressure drop (mixed and free convection) & & $\mathrm{M}$ & & & $\mathrm{M}$ & & \\
\hline f. Radiation heat transfer & & $\mathrm{H}$ & & $\mathrm{H}$ & $\mathrm{H}$ & & \\
\hline g. Gas conduction & & $\mathrm{M}$ & & $\mathrm{M}$ & $\mathrm{M}$ & & \\
\hline h. Bulk CO reaction & & & & $\mathrm{M}$ & $\mathrm{H}$ & & \\
\hline i. Molecular diffusion & & & & $\mathrm{M}$ & & & \\
\hline j. Graphite oxidation (PBR) & & & & $\mathrm{M}$ & & & \\
\hline k. Fluid properties (gas mixture) & & & & & & & \\
\hline
\end{tabular}

Table A-11. Upper Plenum and Components Phenomena Rankings.

\begin{tabular}{|c|c|c|c|c|c|c|c|}
\hline \multirow[b]{2}{*}{ Phenomena } & \multicolumn{2}{|c|}{ PCC } & \multicolumn{3}{|c|}{ DCC } & \multicolumn{2}{|c|}{$\mathrm{LC}$} \\
\hline & 1 & 2 & 1 & 2 & 3 & 1 & 2 \\
\hline a. Flow distribution & $\mathrm{H}$ & M & M & & M & $\mathrm{H}$ & $\mathrm{H}$ \\
\hline b. Heat transfer (forced convection) & M & & M & & & & \\
\hline $\begin{array}{l}\text { c. Heat transfer (mixed and free } \\
\text { convection) }\end{array}$ & & M & & M & M & & \\
\hline d. Pressure drop (forced convection) & M & & $\mathrm{M}$ & & & $\mathrm{H}$ & $\mathrm{H}$ \\
\hline $\begin{array}{l}\text { e. Pressure drop (mixed and free } \\
\text { convection) }\end{array}$ & & M & & & M & & \\
\hline f. Thermal mixing and stratification & & $\mathrm{H} / \mathrm{M}$ & & M & $\mathrm{H}$ & & \\
\hline g. Hot plumes & & $\mathrm{H}$ & & & $\mathrm{H}$ & & \\
\hline i. Fluid properties (G.M) & & & M & M & & & \\
\hline $\begin{array}{l}\text { j. Thermal resistance/heat capacity } \\
\text { of shroud }\end{array}$ & & $\mathrm{H}$ & & & $\mathrm{H}$ & & \\
\hline k. Bulk CO reaction & & & & M & & & \\
\hline 1. Molecular diffusion & & & & M & & & \\
\hline m. Graphite oxidation (PBR) & & & & & & & \\
\hline
\end{tabular}


NEXT GENERATION NUCLEAR PLANT

METHODS RESEARCH AND

DEVELOPMENT TECHNICAL

PROGRAM PLAN
Identifier: PLN-2498

Revision: $\quad 1$

Effective Date: $9 / 25 / 08 \quad$ Page: 149 of $\mathbf{1 8 8}$

Table A-12. Lower Plenum and Components Phenomena Rankings.

\begin{tabular}{|l|c|c|c|c|c|c|c|}
\hline \multirow{2}{*}{\multicolumn{1}{c|}{ Phenomena }} & \multicolumn{2}{c|}{ PCC } & \multicolumn{3}{c|}{ DCC } & \multicolumn{2}{c|}{ LC } \\
\cline { 2 - 8 } \multicolumn{1}{c|}{} & 1 & 2 & 1 & 2 & 3 & 1 & 2 \\
\hline a. Flow distribution & $\mathrm{H}$ & $\mathrm{H}$ & $\mathrm{M}$ & & $\mathrm{H}$ & $\mathrm{H}$ & $\mathrm{H}$ \\
\hline b. Heat transfer (forced convection) & $\mathrm{M}$ & & $\mathrm{M}$ & & & $\mathrm{H}$ & $\mathrm{H}$ \\
\hline c. Heat transfer (mixed and free convection) & & $\mathrm{M}$ & & $\mathrm{M}$ & $\mathrm{M}$ & & \\
\hline d. Pressure drop (forced convection) & $\mathrm{M}$ & & $\mathrm{H}$ & & & $\mathrm{H}$ & $\mathrm{H}$ \\
\hline e. Pressure drop (mixed and free convection) & & $\mathrm{M}$ & & $\mathrm{M}$ & $\mathrm{M}$ & & \\
\hline f. Thermal mixing and stratification & & $\mathrm{H}$ & & $\mathrm{H}$ & $\mathrm{H}$ & & \\
\hline g. Jet discharge & & & & & & $\mathrm{H}$ & $\mathrm{H}$ \\
\hline h. Thermal striping & & & & & & $\mathrm{H}$ & $\mathrm{H}$ \\
\hline i. Bulk CO reaction & & & & $\mathrm{M}$ & $\mathrm{M}$ & & \\
\hline j. Molecular diffusion & & & & $\mathrm{H}$ & & & \\
\hline k. Fluid properties (gas mixture) & & & & $\mathrm{H}$ & $\mathrm{H}$ & & \\
\hline \multicolumn{1}{|c|}{ 1. Graphite oxidation (PBR) } & & & & $\mathrm{H}$ & $\mathrm{H}$ & & \\
\hline
\end{tabular}

\section{A-5.2 Reactor Coolant Loop (RCL) Components}

Table A-13. Hot/Cold Pipe Phenomena Rankings.

\begin{tabular}{|l|c|c|c|c|c|c|c|}
\hline \multirow{2}{*}{\multicolumn{1}{c|}{ Phenomena }} & \multicolumn{2}{c|}{ PCC } & \multicolumn{2}{c|}{ DCC } & \multicolumn{2}{c|}{ LC } \\
\cline { 2 - 7 } & 1 & 2 & 1 & 2 & 3 & 1 & 2 \\
\hline a. Heat transfer (forced convection) & $\mathrm{M}$ & & $\mathrm{M}$ & & & $\mathrm{H}$ & $\mathrm{H}$ \\
\hline b. Heat transfer (mixed and free convection) & & & & & $\mathrm{M}$ & & \\
\hline c. Pressure drop (forced convection) & $\mathrm{M}$ & & $\mathrm{H}$ & & & & \\
\hline d. Pressure drop (mixed and free convection) & & & & & $\mathrm{M}$ & & \\
\hline e. Pipe/insulator conduction & & & & & & & $\mathrm{M}$ \\
\hline f. Critical flow & & & $\mathrm{H}$ & & & & \\
\hline g. Bulk CO reaction & & & & & & & \\
\hline h. Fluid properties / gas mixture & & & & $\mathrm{H}$ & $\mathrm{H}$ & & \\
\hline
\end{tabular}


NEXT GENERATION NUCLEAR PLANT

METHODS RESEARCH AND

DEVELOPMENT TECHNICAL

PROGRAM PLAN
Identifier: $\quad$ PLN-2498

Revision: $\quad 1$

Effective Date: 9/25/08 Page: 150 of $\mathbf{1 8 8}$

\section{A-5.3 Reactor Cavity Cooling System (RCCS) Components}

Table A-14. RCCS Phenomena Rankings.

\begin{tabular}{|c|c|c|c|c|c|c|c|}
\hline \multirow[b]{2}{*}{ Phenomena } & \multicolumn{2}{|c|}{ PCC } & \multicolumn{3}{|c|}{ DCC } & \multicolumn{2}{|c|}{$\mathrm{LC}$} \\
\hline & 1 & 2 & 1 & 2 & 3 & 1 & 2 \\
\hline a. Flow distribution & & $\mathrm{H}$ & & $\mathrm{H}$ & $\mathrm{H}$ & & \\
\hline b. Heat transfer (mixed and free convection) & & $\mathrm{H}$ & & $\mathrm{H}$ & $\mathrm{H}$ & & \\
\hline c. Pressure drop (mixed and free convection) & & M & & M & M & & \\
\hline d. Radiation heat transfer & & $\mathrm{H}$ & & $\mathrm{H}$ & $\mathrm{H}$ & & \\
\hline e. Gas conduction & & M & & M & M & & \\
\hline f. Conduction to ground & & M & & M & M & & \\
\hline g. Dust from core & & & $\mathrm{H}$ & & & & \\
\hline h. Air purge and gas species distribution & & & $\mathrm{H}$ & $\mathrm{H}$ & $\mathrm{H}$ & & \\
\hline i. Fluid properties (gas mixture) & & & $\mathrm{H}$ & $\mathrm{H}$ & $\mathrm{H}$ & & \\
\hline
\end{tabular}

Table A-15. RCCS Tube (Air Duct) Phenomena Rankings.

\begin{tabular}{|c|c|c|c|c|c|c|c|}
\hline \multirow[b]{2}{*}{ Phenomena } & \multicolumn{2}{|c|}{ PCC } & \multicolumn{3}{|c|}{ DCC } & \multicolumn{2}{|c|}{$\mathrm{LC}$} \\
\hline & 1 & 2 & 1 & 2 & 3 & 1 & 2 \\
\hline a. Heat transfer (forced convection) & & $\mathrm{H}$ & & $\mathrm{H}$ & $\mathrm{H}$ & & \\
\hline b. Heat transfer (mixed and free convection) & & $\mathrm{H}$ & & $\mathrm{H}$ & $\mathrm{H}$ & & \\
\hline c. Pressure drop (forced convection) & & $\mathrm{H}$ & & $\mathrm{H}$ & $\mathrm{H}$ & & \\
\hline d. Pressure drop (mixed and free convection) & & $\mathrm{H}$ & & $\mathrm{H}$ & $\mathrm{H}$ & & \\
\hline e. Radiation heat transfer & & $\mathrm{H}$ & & $\mathrm{H}$ & $\mathrm{H}$ & & \\
\hline f. Fluid properties (humidity) & & M & & M & M & & \\
\hline
\end{tabular}

Table A-16. RCCS Piping and Chimney Phenomena Rankings.

\begin{tabular}{|c|c|c|c|c|c|c|c|}
\hline \multirow[b]{2}{*}{ Phenomena } & \multicolumn{2}{|c|}{ PCC } & \multicolumn{3}{|c|}{ DCC } & \multicolumn{2}{|c|}{$\mathrm{LC}$} \\
\hline & 1 & 2 & 1 & 2 & 3 & 1 & 2 \\
\hline a. Heat transfer (mixed and free convection) & & M & & M & M & & \\
\hline b. Pressure drop (mixed and free convection) & & $\mathrm{H}$ & & $\mathrm{H}$ & $\mathrm{H}$ & & \\
\hline c. Flow mixing in piping plenums & & $\mathrm{M}$ & & M & M & & \\
\hline d. Buoyancy flow in chimney & & $\mathrm{H}$ & & $\mathrm{H}$ & $\mathrm{H}$ & & \\
\hline e. Fluid properties (humidity) & & M & & M & M & & \\
\hline
\end{tabular}




\begin{tabular}{|c|lll|}
\hline NEXT GENERATION NUCLEAR PLANT & Identifier: & PLN-2498 & \\
METHODS RESEARCH AND & Revision: & 1 & \\
DEVELOPMENT TECHNICAL & Effective Date: & $9 / 25 / 08$ & Page: 151 of 188 \\
PROGRAM PLAN & & & \\
\hline
\end{tabular}

\section{A-5.4 Power Conversion Unit (PCU) Components}

Table A-17. PCU (Power Conversion Unit) Phenomena Rankings.

\begin{tabular}{|lcrrrrrc|}
\hline & \multicolumn{2}{c}{ PCC } & DCC & & LC \\
\cline { 2 - 7 } \multicolumn{1}{c}{ Phenomena } & 1 & 2 & 1 & 2 & 3 & 1 & 2 \\
\hline a. Turbine performance & $\mathrm{H}$ & & & & & $\mathrm{H}$ & $\mathrm{H}$ \\
\hline b. Turbine valve performance & $\mathrm{H}$ & & & $\mathrm{H}$ & $\mathrm{H}$ \\
\hline c. Recuperator performance & $*$ & & $\mathrm{H}$ & $\mathrm{H}$ \\
\hline d. Coolers performance & & $\mathrm{H}$ & $\mathrm{H}$ \\
\hline e. Compressor performance & $\mathrm{H}$ & $\mathrm{H}$ & $\mathrm{H}$ \\
\hline
\end{tabular}

\section{A-6. DESCRIPTION OF IDENTIFIED PHENOMENA}

Table A-18 presents a list of phenomena in the VHTR and the generic issues that are thought to be important for each. The right-hand side of the table shows the components for which these phenomena are believed to be important, effecting the generation, removal, and storage of energy. One shortcoming of Table A-18, however, is that it neglects the dependence of the importance of a phenomenon in a component on the transient event. Thus, phenomena must be considered with respect to both component and transient event.

The following conventions are adopted. The coastdown and conduction cooldown phases are demarcated by the transition from forced to natural convection heat transfer. The fluid properties phenomenon refers to the species mix of the coolant. The mix is dependent on species transport processes including air ingress into the break, movement of air through the vessel and piping, and consumption of oxygen by oxidation of graphite. The properties associated with a particular gas species are assumed lumped into the heat transfer and pressure drop phenomena. Thus, fluid properties takes in the changing species mix associated with air ingress, movement of air into the system, and consumption of oxygen. The flow distribution phenomenon refers to the macroscopic paths where the coolant is going. On the other hand, the molecular diffusion phenomenon refers to the time evolution of a species spatial concentration. Decay heat is broken into three components, the normalized axial and radial power profiles, a time varying scale factor, and the initial stored energy. It is assumed the plant design is based on confinement, not containment. The homogeneous chemical reaction refers to the migration of low temperature oxygen into the bulk graphite and its reaction with the graphite. Graphite oxidation is a surface phenomenon where high temperature oxygen reacts with the exposed graphite. We do not consider the intermediate heat exchanger that provides heat to the hydrogen plant, the ingress of water due to a shutdown cooling system leak, or an indirect cycle arrangement of components.

\section{A-6.1 Pressured Conduction Cooldown (PCC) Event}

The PCC event is initiated by a loss of the main heat transport system coolers while the plant is at the full power condition. This is followed by a reactor trip and the failure of the Shutdown Cooling System to start. With the loss of the main coolers the capability of the primary system to function as a heat engine is lost. The turbomachine coasts down until it stops at which point there is no longer forced circulation through the core. Heat removal from the core is by the RCCS. The PCC thus has two distinct phases, (1) the coastdown phase and (2) the heat-up and passive cooling phase. The important phenomena for each component are described below. 


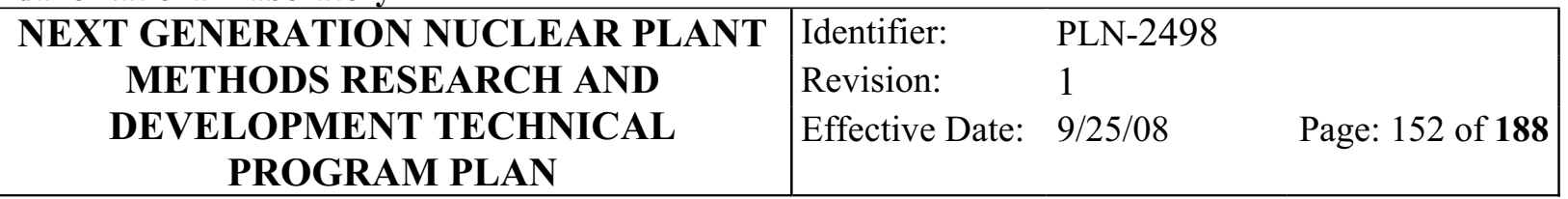

- Inlet Plenum. The inlet plenum feeds the risers during the coastdown phase and has an impact on the distribution of flow among the risers. The proportioning of flow among the risers during coastdown will change if the inlet plenum is not large enough to preclude pressure drop around its azimuthal periphery. A non-negligible pressure drop in turn will change the flow distribution in the core. The effect is not as great for the PBR since the pebbles tend to diffuse any flow maldistribution as the coolant moves down the core. This phenomenon ceases to be a factor once coastdown is complete. At that time the inlet plenum ceases to participate hydraulically since its flow is stagnant.

- Riser. The risers are a set of parallel channels that connect the inlet plenum to the upper plenum. Their hydraulic characteristics will effect the re-proportioning of coolant among them during the coastdown phase of the transient. This phenomenon ceases to be a factor once coastdown is complete. At that time the risers cease to participate hydraulically since the flow is stagnant. The risers are gas filled channels distributed around the periphery of the vessel. During conduction cooldown radiation heat transfer and gas conduction may be important components in the radial flow of heat to the vessel wall. There may be internal recirculation paths established with each riser, aiding heat transfer. The possibility of no net flow but alternating up flow and down flow between the inlet and upper plenums exists.

- Upper Plenum and Components. During forced circulation the upper plenum distributes coolant to the core. The volume of the plenum is an important factor in the manner in which flow from the risers distributes itself during the coastdown. When coastdown is complete the upper plenum connects the core and reflector channels with the lower plenum establishing a network of parallel passages. The coolant in the hotter channels exits the core upward in buoyant plumes while flow in the cooler channels moves down discharging into the lower plenum. The hot fluid entering the upper plenum may stratify in the absence of active mixing. The heat capacity and the thermal resistance of the thermal shroud will be important factors in the temperatures seen by the vessel at the upper head. The heat transfer to the shroud will be both radiative from the top of the core and mixed and natural convection from the gas in the plenum.

- Core and Reflector. There are a number of important phenomena during both the coastdown and conduction cooldown phases. The fraction of coolant that bypasses the core coolant channels in the prismatic modular reactor (PMR) is perhaps the most important phenomenon for fuel temperature. The fraction is related to core configuration and is dependent on fuel block dimensional changes over life. In the PMR fueled blocks stacked one on top of the other may have gaps between them that causes flow diversion. In the PBR flow diversion is less important because the leakage paths among fueled balls over life can be better predicted. However, the pebbles in contact with the vessel wall will have an average porosity that is greater than the bulk. This gives rise to a multi-dimensional heat distribution and bypass overcooling. The flow distribution through the PMR core is important as it affects the thermal conditions in the hot channel. Transition from coastdown to cooldown may be marked by flow stoppage in some channels as flow undergoes reversal. The PMR core contains a multi-dimensional arrangement of coolant channels within a block that may require detailed spatial to locate the local hot spot. While a porous body modeling of the fluid is not suited to the PMR, it may be adequate for the PBR. In addition the neutronic power may need to be computed on a block-byblock basis to properly resolve the radial heating profile. This profile is important for the flow distribution by natural convection in the cooldown phase. The temporal behavior of the decay heat curve is important since it affects the heat load seen by the heat removal systems. Fluid properties, particularly viscosity, are important during both coastdown and cooldown. Viscosity increases with temperature. During cooldown the coolant flowing in the hottest channel will have the greatest viscosity which will decrease cooling in that channel and increase fuel temperature. The core heat 


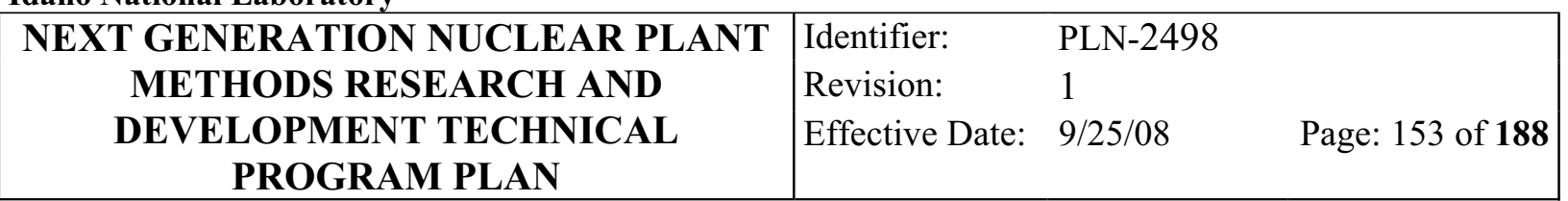

capacity is very important as it is a main mechanism for storing heat that otherwise would be removed through a heat transport path. Fuel temperatures would otherwise be greater. The gaps between blocks in the same plane and the associated gap conductance are important to radial heat transfer in the PMR. Under core configuration, the pebbles in the PBR may pile up locally at the top of core affecting the core power distribution.

- Lower Plenum and Components. During the coastdown phase the hot core coolant enters the lower plenum. In the PMR the coolant enters as a hot jet before it diffuses within the plenum. Thermal striping on components is an issue. Thermal stripping is less an issue in the PBR since the coolant flow paths are connected laterally and since the flow amongst the balls gives rise to converging and diverging jets, both of which give rise to better mixing before the coolant exits the core into he plenum. The plenum connects the vertical coolant channels in which parallel circulation patterns develop within the core during cooldown. These patterns are a principle means for moving energy from the core interior toward to vessel wall. The inlet plenum thus plays a role in the mixing and stratification in the upper plenum.

- Hot/Cold Pipe. The leakage of heat by conduction from the hot inner pipe to the cool outer annulus and the related performance of the insulation are the main phenomena. The hot and cold pipes in the PBR are much longer than the PMR so conduction phenomenon may be more important in the PBR. The hot-cold pipes may act as a heat pipe transporting heat away from the vessel and radiating it to the reactor cavity. The transport may be a combination of convection and conduction.

- Reactor Cavity. Heat removal by the RCCS during PCC is the main path for cooling the vessel. The radial temperature gradient developed across the core heat to the vessel. The transfer of heat from the vessel wall to the air ducts is mainly by radiation heat transfer. Computer code calculations with CFD models indicate that this is 90 percent of the heat transfer with convection by air in the cavity making up the balance. The view factors for the reactor vessel communicating with the ducts are especially complex because the vessel geometry is circular while the duct layout is Cartesian. The impact of simplifications used in computing view factors must be quantified. In the cooldown phase the hot plumes in the vessel head raise the temperature of the vessel wall at the top such that the vessel temperature and not the fuel may be the limiting condition.

- RCCS Air Duct. The energy conducted through the duct walls from the reactor cavity is convected to the air inside the duct and is also radiated by the inner surfaces of the duct to adjacent surfaces. A buoyant head is established inside the duct as the air heated by the duct walls expands, rises, and draws air in at the duct inlet. The heat transfer and pressure loss phenomena inside the duct are dependant on the velocity profile at the wall. If local buoyancy at the wall is induced, then the heat transfer and pressure loss processes operate in the mixed rather than forced convection mode. Because of the non-circular geometry of the duct and non-uniform heat flux at the duct surface, modeling of the mixed convection region requires special treatment. Either scaled experiments are required to obtain integral data or a validated CFD code is needed to solve explicitly for the flow field in the duct interior. Recent results suggest that fluid property variation may be more important than the mode of convection.]

- RCCS Piping and Chimney. The same considerations for the air duct apply here also. The effect of flow mixing in plena connecting pipes is considered to have negligible impact on fuel and vessel temperature because it is so far removed from the vessel and fuel.

- Power Conversion Unit. The role of the PCU diminishes as the turboshaft coasts down. While the time length for coastdown may be a factor that determines which phenomena are important, presently 


\begin{tabular}{|c|lll|}
\hline NEXT GENERATION NUCLEAR PLANT & Identifier: & PLN-2498 \\
METHODS RESEARCH AND & Revision: & 1 & \\
DEVELOPMENT TECHNICAL & Effective Date: & $9 / 25 / 08$ & Page: 154 of 188 \\
PROGRAM PLAN & & & \\
\hline
\end{tabular}

the coastdown time is not known. There is a possibility for generation of pressure waves in the PCU as a result of compressor stall.

\section{A-6.2 Depressurized Conduction Cooldown (DCC) Event}

The DCC event is initiated by a guillotine break of the hot/cold pipe. The coolant inventory blows down, exiting through the opening in the inner hot duct and in the annular cold duct. The PCU is effectively isolated from the reactor vessel and plays no significant role in the event. The reactor trips on loss of pressure. After blowdown, air begins to diffuse into the primary system through the site of the break. If sufficient air enters the primary system, natural circulation paths are established. The DCC has three distinct phases, (1) blowdown, (2) molecular diffusion, and (3) natural convection.

- Inlet Plenum. During the blowdown phase, depending on the relative volumes of the vessel flow passages, flow reversal may bring hot core coolant into the inlet plenum. During the air ingress phase, air will tend to enter at the bottom of the annular cold pipe. Acoustic noise will aid its diffusion into the plenum. The molecular diffusion of air is very dependent on the gas temperature in the plenum. Thus, thermal stratification and mixing in the inlet plenum must be first well understood before air ingress can be predicted reliably. Large thermal gradients in the inlet plenum will enhance or inhibit molecular diffusion of air into the plenum. The progression of the air ingress phase has a significant effect on the natural convection phase.

- Riser. During the air ingress phase the temperature distribution in the riser is important to the progression of air diffusion. Thus, the role of thermal stratification in the riser must be well understood. In the PBR the risers contain graphite and which may act as a parasitic sink for oxygen during the air ingress and natural convection phases. The shortest path to the core is, however, through the lower plenum. Thus, the risers may have a reduced role in the progression of the air ingress phase. In the natural convection stage, depending on the gas mixture, there is a possibility for recirculation flow within the riser of alternating up and down flow in adjacent risers. This will enhance the transport of heat from the core to the vessel wall.

- Upper Plenum and Components. The potential for graphite oxidation in the natural convection phase depends on how well air infiltrated in the previous phase.

- Core and Reflector. Reaction forces during the blowdown phase can load vessel structures and loosen graphite dust previously created by mechanical friction of fuel elements. The PBR will have higher dust levels because the fuel is in motion. The blowdown will carry some of the dust and vent it into containment or confinement. The confinement must be equipped with a filter to prevent discharge of the dust to the environment. During the blowdown phase, the helium in the core is cooled by the mechanical work of decompression. This cooling term may serve to lower fuel temperature from what it would otherwise be. The ingress of air creates the potential for significant oxidation of the fuel. During the natural convection phase, the circulating gas will aid in the removal of the heat of oxidation. The degree of oxidation is related to available supply of air during the ingress phase. For confinement, the original air in the reactor cavity will be partially displaced by helium from the break. For containment, the available air is a function of the volume of the containment. The air flowrate into the vessel during the natural convection phase likely will be insufficient to significantly cool the core. It will however generate significant heat of oxidation, raising structure temperatures, as it reacts with the graphite. The main path for air ingress is through the lower plenum via the hot duct. The graphite structures below the fueled region of the core will react with the air and reduce the concentration of oxygen entering the fueled region of the core. Graphite oxidation will be more 


\begin{tabular}{|c|lll|}
\hline NEXT GENERATION NUCLEAR PLANT & Identifier: & PLN-2498 \\
METHODS RESEARCH AND & Revision: & 1 & \\
DEVELOPMENT TECHNICAL & Effective Date: & $9 / 25 / 08$ & Page: 155 of 188 \\
PROGRAM PLAN & & & \\
\hline
\end{tabular}

important in the PBR as a consequence of the greater fuel surface area and resulting higher oxidation rate.

- Lower Plenum and Components. The lower plenum lies between the site of the break and the core and is thus in the shorter of two paths that lead from the reactor cavity to the core. Consequently, during the blowdown phase, graphite dust lodged in the lower core and the lower plenum may be swept through the lower plenum and out the break into the confinement. During the air ingress phase, the thermal stratification in the lower plenum will play a role in the rate of molecular diffusion of air into the core. If graphite is present in the lower plenum, it will act as a site for oxidation and may result in reduced concentrations reaching the reactor fuel. During the natural convection phase the lower plenum may be important to the flow patterns that are established.

- Hot/Cold Pipe. The double guillotine of the hot/cold pipe creates two separate paths from the reactor cavity to the core, one through the inlet plenum and one through the lower plenum. As mentioned in [7] blowdown will be through both paths with the result that a plane of zero velocity will exist at some point, likely in the core. The reactor cavity conditions beginning with the start of the air ingress phase defines the air concentration boundary condition at the break.

- Reactor Cavity. The air in the reactor cavity before the onset of the upset will contain water vapor. Some of this water vapor will be present in the mixture of gases that enter the break site during the air ingress phase. In addition, the PBR may include a source of cooling water that might enter the reactor cavity and, during the air ingress and natural convection phases, enter the reactor vessel as water vapor through the break. During the blowdown phase, graphite dust may be discharged into the reactor cavity. If the cavity acts as a confinement, then the release of this radioactive dust into the environment through a relief valve must be considered. The dust may settle on RCCS heat transfer surface in the cavity changing their heat transfer characteristics. During the air ingress and natural convection phases, some the air in the cavity that was not displaced will move into the reactor vessel and oxidize graphite surfaces.

- RCCS Air Duct. These are the same consideration as for the DCC. Additionally, one must also consider possible collapse of the air duct walls by pressure difference during blowdown..

- RCCS Piping and Chimney. These are the same phenomena that are important during the PCC.

- Power Conversion Unit. The power conversion unit is effectively isolated from the reactor vessel after blowdown and plays no significant role in the event.

\section{A-6.3 Load Change (LC) Event}

The LC event is initiated with a reduction in primary flowrate by 20 percent over a 20 second period and is then maintained at this value. The reactor is not tripped. The net reactivity is assumed to return to zero in the asymptote through natural temperature change without control rod motion. The PCC has two distinct phases, (1) the flow transient phase followed by transition to a new equilibrium condition (2) and the new steady-state operating condition. The plant response is highly dependent on the behavior of the plant control system. Assuming the reactor inlet temperature is maintained constant and Doppler and coolant feedback are the main reactivity temperature effects, the reduction in cooling will raise the average core temperature which will reduce the core power. Whether the reactor outlet temperature increases or decreases depends on the coolant to fuel temperature rise and the coolant to Doppler reactivity feedbacks. 


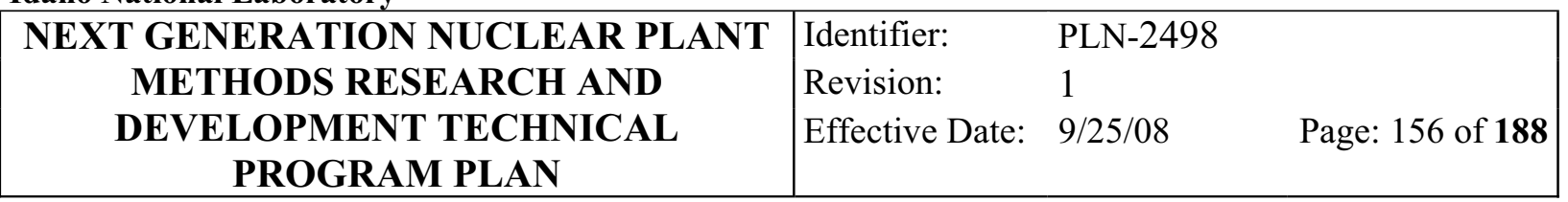

It is expected, however, that the core outlet temperature will change and that will lead to the movement of local hot spots. The main concern is the relocation of a structure hot spot during the transient and the thermal stresses generated. The temperature field at the turbine inlet piping has been suggested as an area for concern. The important phenomena for each component are described below.

- Upper Plenum and Components. The flow will redistribute in the upper plenum so that the flowrate into the PMR core channels will change.

- Core and Reflector. The redistribution of flow among the core channels will change the location of the core hot spot.

- Lower Plenum and Components. The importance of thermal striping will be greater for the PMR since the coolant streams exiting the PMR core have a wider temperature variation because there is less mixing as the coolant flows down the core. The coolant may exhibit a temperature gradient in the plane that is normal to the flow of coolant through the lower plenum. This is because the coolant exiting the core is not isothermal.

- Hot/Cold Pipe. The hot pipe takes coolant from the inlet plenum. The location of the hot spot in the hot pipe will change as the flow pattern in the lower plenum changes with the flow reduction.

- Power Conversion Unit. The turbomachine performance will effect the temperatures exiting the turbomachines and hence the temperatures seen by the recuperator.

Table A-18. Summary of Identified Phenomena.

\begin{tabular}{|l|l|l|}
\hline \multicolumn{1}{|c|}{ Phenomena } & \multicolumn{1}{|c|}{ Issue } & \multicolumn{1}{|c|}{$\begin{array}{c}\text { Associated } \\
\text { Components }\end{array}$} \\
\hline Flow distribution & $\begin{array}{l}\text { Nonuniform and asymmetric flow may result in local hot spot } \\
\text { in core } \\
\text { Natural circulation inside core is one of core cooling } \\
\text { mechanism during PCC and DCC } \\
\text { Natural circulation inside reactor cavity partly contributes to } \\
\text { RCCS heat removal } \\
\text { Bypass flow }\end{array}$ & $\begin{array}{l}\text { Core/Reflector } \\
\text { Plenums } \\
\text { Riser } \\
\text { RCL } \\
\text { Rx Cavity }\end{array}$ \\
\hline Fluid properties & $\begin{array}{l}\text { Accurate prediction of gas properties is a basic requirement for } \\
\text { analyzing gas flow and heat transfer }\end{array}$ & $\begin{array}{l}\text { Core/Reflector } \\
\text { Plenums } \\
\text { Riser } \\
\text { RCL } \\
\text { RCCS }\end{array}$ \\
\hline $\begin{array}{l}\text { Convection Heat } \\
\text { transfer }\end{array}$ & $\begin{array}{l}\text { Accurate representation of heat transfer is to ensure adequate } \\
\text { heat removal rate } \\
\text { Heat transfer regime tends to be in mixed or free convection } \\
\text { heat transfer during accident conditions }\end{array}$ & $\begin{array}{l}\text { Core/Reflector } \\
\text { Plenums } \\
\text { Riser } \\
\text { Loop } \\
\text { RCCS } \\
\text { PCU }\end{array}$ \\
\hline
\end{tabular}




\begin{tabular}{|c|lll|}
\hline NEXT GENERATION NUCLEAR PLANT & Identifier: & PLN-2498 \\
METHODS RESEARCH AND & Revision: & 1 & \\
DEVELOPMENT TECHNICAL & Effective Date: & $9 / 25 / 08$ & Page: 157 of 188 \\
PROGRAM PLAN & & & \\
\hline
\end{tabular}

\begin{tabular}{|c|c|c|}
\hline Phenomena & Issue & $\begin{array}{l}\text { Associated } \\
\text { Components }\end{array}$ \\
\hline Pressure drop & $\begin{array}{l}\text { Accurate representation of pressure drop is to ensure adequate } \\
\text { design flow rate and flow distribution }\end{array}$ & $\begin{array}{l}\text { Core/Reflector } \\
\text { Plenums } \\
\text { Riser } \\
\text { Loop } \\
\text { RCCS } \\
\text { PCU } \\
\end{array}$ \\
\hline $\begin{array}{l}\text { Radiation heat } \\
\text { transfer }\end{array}$ & $\begin{array}{l}\text { Accurate representation of radiation heat transfer in a complex } \\
\text { geometry is to ensure adequate heat removal from core to } \\
\text { RCCS }\end{array}$ & $\begin{array}{l}\text { Core/Reflector } \\
\text { Riser } \\
\text { RCCS }\end{array}$ \\
\hline $\begin{array}{l}\text { Contact heat } \\
\text { transfer }\end{array}$ & $\begin{array}{l}\text { Accurate representation of contact heat transfer in a complex } \\
\text { geometry is to ensure adequate heat removal from core to } \\
\text { RCCS }\end{array}$ & $\begin{array}{l}\text { Core/Reflector } \\
\text { RCCS }\end{array}$ \\
\hline $\begin{array}{l}\text { Gas conduction heat } \\
\text { transfer }\end{array}$ & $\begin{array}{l}\text { Accurate representation of gas conduction is to ensure adequate } \\
\text { heat removal from core to RCCS }\end{array}$ & $\begin{array}{l}\text { Core/Reflector } \\
\text { Rx cavity } \\
\text { Riser }\end{array}$ \\
\hline $\begin{array}{l}\text { Thermal mixing and } \\
\text { stratification and } \\
\text { gas species } \\
\text { stratification }\end{array}$ & $\begin{array}{l}\text { Thermal stratification in the plenums is a challenge to material } \\
\text { integrity and core local hot spot }\end{array}$ & $\begin{array}{l}\text { Plenums } \\
\text { Hot/Cold Piping } \\
(\text { PBR!) }\end{array}$ \\
\hline $\begin{array}{l}\text { Material properties } \\
\text { of fuel and reflector }\end{array}$ & $\begin{array}{l}\text { Accurate representation of conductivity and specific heat of } \\
\text { fuel and reflector is to ensure local temperature distribution. } \\
\text { Effects of irradiation on the material properties are especially } \\
\text { important in affecting the gap dimensions and consequently the } \\
\text { bypass flow. }\end{array}$ & Core/Reflector \\
\hline Core decay heat & $\begin{array}{l}\text { Core power is the heat source that determines the consequent } \\
\text { temperature transients }\end{array}$ & Core/Reflector \\
\hline Reactivity feedback & Doppler feedback provides intrinsic safety features & Core/Reflector \\
\hline $\begin{array}{l}\text { Multi-dimensional } \\
\text { heat conduction }\end{array}$ & $\begin{array}{l}\text { Accurate prediction of local temperature distribution in a } \\
\text { complex geometry is to ensure the local hot spot and reactivity } \\
\text { feedback }\end{array}$ & Core/Reflector \\
\hline Hot plume & $\begin{array}{l}\text { Hot plume from core in the upper plenum is a challenge to } \\
\text { vessel integrity }\end{array}$ & Upper Plenum \\
\hline $\begin{array}{l}\text { Thermal } \\
\text { resistance/heat } \\
\text { capacity of shroud }\end{array}$ & $\begin{array}{l}\text { Thermal resistance and heat capacity of shroud in upper } \\
\text { plenum is to protect vessel overheat }\end{array}$ & Upper Plenum \\
\hline Core configuration & $\begin{array}{l}\text { Location of pebble; pebble void fraction as function of location } \\
\text { and pebble trajectories. } \\
\text { Gaps between the blocks }\end{array}$ & Core/Reflector \\
\hline Jet discharge & $\begin{array}{l}\text { Jet discharge into outlet plenum can induce spatial and } \\
\text { temporal variations in temperature of the plenum wall, which } \\
\text { may result in material fatigue } \\
\text { Momentum of impinging stream may damage insulation }\end{array}$ & Outlet Plenum \\
\hline
\end{tabular}


Idaho National Laboratory

\begin{tabular}{|c|lll|}
\hline NEXT GENERATION NUCLEAR PLANT & Identifier: & PLN-2498 & \\
METHODS RESEARCH AND & Revision: & 1 & \\
DEVELOPMENT TECHNICAL & Effective Date: & $9 / 25 / 08$ & Page: 158 of 188 \\
PROGRAM PLAN & & & \\
\hline
\end{tabular}

\begin{tabular}{|c|c|c|}
\hline Phenomena & Issue & $\begin{array}{l}\text { Associated } \\
\text { Components }\end{array}$ \\
\hline Thermal striping & $\begin{array}{l}\text { High frequency temperature change by jet discharge can induce } \\
\text { material fatigue }\end{array}$ & Outlet Plenum \\
\hline $\begin{array}{l}\text { Pipe/insulator } \\
\text { conduction }\end{array}$ & $\begin{array}{l}\text { Accurate prediction of thermal resistance of pipe/insulator is to } \\
\text { ensure mechanical integrity of hot/cold pipe }\end{array}$ & Hot/cold pipe \\
\hline Critical flow & $\begin{array}{l}\text { Accurate prediction of critical flow is to ensure } \\
\text { depressurization of reactor vessel } \\
\text { The high velocities associated with the large-break may cause } \\
\text { critical flow not only at the hot duct exit but also in some small } \\
\text { area flow passages in the reactor vessel. The influence of the } \\
\text { high flow rates on the redistribution of dust in the reactor } \\
\text { vessel, the PCU, and into the reactor confinement should be } \\
\text { considered. }\end{array}$ & Hot/cold pipe \\
\hline bulk $\mathrm{CO}$ reaction & $\begin{array}{l}\text { Accurate prediction of bulk } \mathrm{CO} \text { reaction is to ensure species } \\
\text { concentration and gas temperature }\end{array}$ & $\begin{array}{l}\text { Core/Reflector } \\
\text { Plenums } \\
\text { Riser }\end{array}$ \\
\hline Graphite oxidation & $\begin{array}{l}\text { Accurate prediction of graphite surface oxidation is to ensure } \\
\text { peak fuel temperature by air-ingress }\end{array}$ & Core/Reflector \\
\hline Molecular diffusion & $\begin{array}{l}\text { Accurate prediction of molecular diffusion in a complex } \\
\text { geometry is to ensure on-set of bulk natural circulation and the } \\
\text { reaction rate of bulk CO and graphite oxidation } \\
\text { Noise affects the diffusion process }\end{array}$ & $\begin{array}{l}\text { Plenums } \\
\text { Riser } \\
\text { Core/Reflector }\end{array}$ \\
\hline $\begin{array}{l}\text { Confinement valve } \\
\text { and filter } \\
\text { characteristics }\end{array}$ & $\begin{array}{l}\text { The confinement relief valve and the filtering hardware } \\
\text { location and performance characteristics are important in } \\
\text { establishing the valve lift interval, fluid discharge } \\
\text { characteristics, and fraction of dust that is retained in } \\
\text { confinement. }\end{array}$ & \\
\hline $\begin{array}{l}\text { Air purge and gas } \\
\text { species distribution }\end{array}$ & $\begin{array}{l}\text { Accurate prediction of gas species distribution in reactor cavity } \\
\text { is to define oxygen supply to reactor vessel }\end{array}$ & Reactor cavity \\
\hline Dust from core & $\begin{array}{l}\text { Graphite dust from the core is source term for fission product } \\
\text { and aerosol transport in confinement }\end{array}$ & Reactor cavity \\
\hline $\begin{array}{l}\text { Conduction to } \\
\text { ground }\end{array}$ & $\begin{array}{l}\text { Conduction to ground is a final success path for core afterheat } \\
\text { removal }\end{array}$ & Reactor cavity \\
\hline $\begin{array}{l}\text { Flow mixing in } \\
\text { piping plenums }\end{array}$ & $\begin{array}{l}\text { Flow mixing in RCCS plenums affects the flow distribution in } \\
\text { RCCS }\end{array}$ & RCCS \\
\hline $\begin{array}{l}\text { Buoyancy flow in } \\
\text { chimney }\end{array}$ & $\begin{array}{l}\text { Accurate prediction of buoyancy flow in chimney is to ensure } \\
\text { RCCS heat removal rate }\end{array}$ & RCCS \\
\hline $\begin{array}{l}\text { Turbine } \\
\text { performance }\end{array}$ & $\begin{array}{l}\text { Accurate representation of turbine performance is to ensure } \\
\text { system response during transients }\end{array}$ & PCU \\
\hline $\begin{array}{l}\text { Compressor } \\
\text { performance }\end{array}$ & $\begin{array}{l}\text { Accurate representation of compressor performance is to } \\
\text { ensure system response during transients }\end{array}$ & PCU \\
\hline
\end{tabular}




\begin{tabular}{|c|lll|}
\hline NEXT GENERATION NUCLEAR PLANT & Identifier: & PLN-2498 & \\
METHODS RESEARCH AND & Revision: & 1 & \\
DEVELOPMENT TECHNICAL & Effective Date: & $9 / 25 / 08$ & Page: 159 of 188 \\
PROGRAM PLAN & & & \\
\hline
\end{tabular}

\begin{tabular}{|l|l|l|}
\hline \multicolumn{1}{|c|}{ Phenomena } & \multicolumn{1}{|c|}{ Issue } & \multicolumn{1}{c|}{$\begin{array}{c}\text { Associated } \\
\text { Components }\end{array}$} \\
\hline Cooler performance & $\begin{array}{l}\text { Accurate representation of heat transfer and pressure drop of } \\
\text { coolers is to ensure system response during transients }\end{array}$ & PCU \\
\hline $\begin{array}{l}\text { Recuperator } \\
\text { performance }\end{array}$ & $\begin{array}{l}\text { Accurate representation of heat transfer and pressure drop of } \\
\text { recuperator is to ensure system response during transients }\end{array}$ & PCU \\
\hline Valve performance & $\begin{array}{l}\text { Accurate representation of valve performance is to ensure } \\
\text { system response during transients }\end{array}$ & PCU \\
\hline $\begin{array}{l}\text { Heat conduction in } \\
\text { thick-walled } \\
\text { structure }\end{array}$ & $\begin{array}{l}\text { Large spatial temperature gradient can lead to large thermal } \\
\text { stress and component fatigue }\end{array}$ & PCU (turbine inlet) \\
\hline Pressure pulses & $\begin{array}{l}\text { Pressure waves that move through the system will stem from: } \\
\text { PCU equipment malfunctions, e.g., compressor surges } \\
\text { A guillotine break of the hot duct or other lines in the system } \\
\text { Pressure waves may cause the dislodgement of dust in the } \\
\text { system and may inadvertently cause an unexpected large } \\
\text { differential pressure to exist in the system. }\end{array}$ & $\begin{array}{l}\text { PCU } \\
\text { Reactor vessel and } \\
\text { components } \\
\text { Reactor cavity }\end{array}$ \\
\hline
\end{tabular}

\section{A-7. OBSERVATIONS}

Preliminary PIRTs have been developed for two reference VHTR designs based on: (1) 600 MWth GT-MHR for the representative PMR and (2) 400 MWth PBMR for the representative pebble-bed reactor (PBR). Three highly-ranked event scenarios were selected as the nucleus set of events: (1) PCC event initiated by loss of flow and simultaneous failure of shutdown cooling system, (2) DCC event initiated by loss of coolant with air-ingress and (3) load changes including normal operation conditions. Each event scenario is subdivided into several time phases reflecting the major thermal-fluid characteristics and processes of the event. For each phase, each component of the reference designs is examined to identify the various phenomena. The phenomena are then ranked with respect to their influence on the selected primary criteria, both safety and otherwise by an expert panel group in Korea and the US. Although three scenarios have been examined and the key phenomena have been prioritized, there are a number of other scenarios which have yet to be considered and evaluated, e.g., the water ingress event, rod withdrawal anticipated transient without scram, etc.

The PIRTs generated in this study are first-cut and intended for use in identifying the experimental database and further experiment needs as well as qualifying and validating the capability of existing analysis tools. A further refinement of the PIRTs is required as the vendor designs become more accurately specified.

\section{A-8. REFERENCES}

W. J. Lee, T. Y. C. Wei, R. R. Schultz, K. D. Kim, H. K. Hwang, M. J. Lim, S. W. Lee, R. B. Vilim, E. E. Feldman, W. D. Pointer, P. D. Bayless, and R. L. Moore, 2005, Generation of a Preliminary PIRT (Phenomena Identification and Ranking Table) for Very High Temperature Gas-Cooled Reactors, KAERI/TR-3050/2005, INL/EXT-05-00829, ANL-GenIV-066, September. 


\begin{tabular}{|c|lll|}
\hline NEXT GENERATION NUCLEAR PLANT & Identifier: & PLN-2498 & \\
METHODS RESEARCH AND & Revision: & 1 & \\
DEVELOPMENT TECHNICAL & Effective Date: & $9 / 25 / 08$ & Page: 160 of 188 \\
PROGRAM PLAN & & & \\
\hline
\end{tabular}

R.B. Vilim, E.E. Feldman, W.D. Pointer, T.Y.C. Wei, 2004, Initial VHTR Accident Scenario Classification: Models and Data, Status Report, ANL, September.

R. R. Schultz, et al., Next Generation Nuclear Plant - Design Methods Development and Validation Research and Development Program Plan, 2004, INEEL/EXT-04-02293, Rev 1, INL, September.

R.R. Schultz, S. Ball, J. King, 2005, Catalogue of Validation Data: Gas-Cooled Reactor Operational and Accident Scenarios and Phenomena Identification and Ranking, INL/EXT-05-00157, March. 


\begin{tabular}{|c|lll|}
\hline NEXT GENERATION NUCLEAR PLANT & Identifier: & PLN-2498 \\
METHODS RESEARCH AND & Revision: & 1 & \\
DEVELOPMENT TECHNICAL & Effective Date: & $9 / 25 / 08$ & Page: 161 of 188 \\
PROGRAM PLAN & & & \\
\hline
\end{tabular}

\section{Appendix B}

\section{Summary of Next Generation Nuclear Plant Methods Workshop, Salt Lake City, UT August 22-23, 2006}


NEXT GENERATION NUCLEAR PLANT

METHODS RESEARCH AND

DEVELOPMENT TECHNICAL

PROGRAM PLAN
Identifier: $\quad$ PLN-2498

Revision: $\quad 1$

Effective Date: 9/25/08 Page: 162 of $\mathbf{1 8 8}$ 


\begin{tabular}{|c|lll|}
\hline NEXT GENERATION NUCLEAR PLANT & Identifier: & PLN-2498 \\
METHODS RESEARCH AND & Revision: & 1 & \\
DEVELOPMENT TECHNICAL & Effective Date: & $9 / 25 / 08$ & Page: 163 of 188 \\
PROGRAM PLAN & & & \\
\hline
\end{tabular}

\section{Appendix B}

\section{Summary of Next Generation Nuclear Plant Methods Workshop, Salt Lake City, UT August 22-23, 2006}

The NGNP Design Methods Workshop was convened in Salt Lake City, Utah on August 22 and 23 to discuss the direction being taken in the methods R\&D planning. The audience was composed of technical staff from the following organizations: Areva Ltd; Westinghouse, Inc, Technology Insights Inc, NRC; Oregon State University, Massachusetts Institute of Technology, University of Idaho, ANL, INL, and Oak Ridge National Laboratory. The experts from these organizations were about equally divided between the reactor physics and thermal fluids areas.

The Workshop progressed by first discussing the "gaps" and then addressing how the gaps might be eliminated or minimized. The "gaps" refer to whether or not the software tools under consideration are capable of calculating the phenomenon of interest. If the software isn't capable of calculating the required phenomenon, then the discussion centered on what sort of planning is ongoing to ensure the software tools under consideration are either replaced by software that can perform the necessary calculations or are being validated adequately.

Following a set of presentations designed to acquaint the workshop participants with the NGNP Methods R\&D planning to date, the workshop was divided into two groups that addressed reactor physics and thermal-fluids behavior respectively. The following subsections discuss reactor physics and thermalfluids behavior in sequence. The summary for each of these technical areas includes points made during the presentations as well as during the divided workshop.

\section{B-1. REACTOR PHYSICS}

The tools discussed under the heading of reactor physics fall into two groups: tools for the calculation of reactor physics parameters and tools for the analysis of pebble flow mechanics. The first group includes tools for both pebble-bed and prismatic reactors, while the second group only includes tools applicable to the pebble-bed. The parameters to be calculated by each group of tools are listed below:

\section{Group I: reactor physics parameters}

- Multiplication factor

- Reactivity worths and swing

- Reactivity coefficients

- Flux distribution

- Fluence distribution

- Power distribution

- Point kinetics parameters

- Fuel burnup distribution

- Nuclide density distribution 


\begin{tabular}{|c|lll|}
\hline NEXT GENERATION NUCLEAR PLANT & Identifier: & PLN-2498 \\
METHODS RESEARCH AND & Revision: & 1 & \\
DEVELOPMENT TECHNICAL & Effective Date: & $9 / 25 / 08$ & Page: 164 of 188 \\
PROGRAM PLAN & & & \\
\hline
\end{tabular}

\section{Group II: Pebble Flow Mechanics}

- Pebble flow pattern

For each of the parameters listed above, comments were recorded for each of the following categories of tools:

- ENDF data processing tools

- Stochastic capabilities

- Lattice-cell tools (for preparation of multi-group cross sections)

- Whole-core analysis tools (multi-step design approach)

- Whole-core analysis tools (spatially heterogeneous modeling)

- Source term/decay heat determination

- Coupled neutron-gamma calculation

- Spatial kinetics calculation

- Integral data for validation.

In the above list, the order is the same as in Appendix F of Parker and West 2006 except that the category of integral data for validation is moved to the final position because it is relevant to all of the other categories, and because it differs from them in being a set of data instead of a set of computational tools.

The same comments usually apply to a given computational tool regardless of which parameter the tool is being applied to calculate. For example, MCNP is inefficient for repetitive design optimization calculations, because it takes a long time to complete a run for complicated problems. This is true whether MCNP is being used to find k-effective or flux distributions. Therefore, most of the comments appear in the section on the multiplication factor (the first parameter considered), and subsequent sections allude to comments on the multiplication factor. Therefore, it is more concise to summarize the comments under the headings of tool categories rather than under the headings of the parameter types. The comments are summarized below.

\section{B-1.1 ENDF Data Processing Tools}

The participants note that processing codes such as NJOY and AMPX are well established, well maintained, and universally recognized tools, and that they are generally adequate for processing ENDF/B data. Some data gaps are noted, especially in graphite cross sections, plutonium, and some of the higher actinides. New evaluations and measurements are needed as identified in the current DOE Physics Working Group priorities. It is also noted that improved covariance matrices are needed for a large number of nuclides important for the VHTR. The treatment of scattering in the resonance region needs more attention.

Two separate comments lament the low level of funding given to these issues. One of the comments criticizes INL and DOE upper management for not recognizing the importance of design and evaluation methods in general or the potential savings they offer as alternatives to expensive physical mockups. This comment is repeated for every tool category. Evidently, methods development efforts are hampered by inadequate funding support. Other comments point out that the fuels and materials issues are the critical 


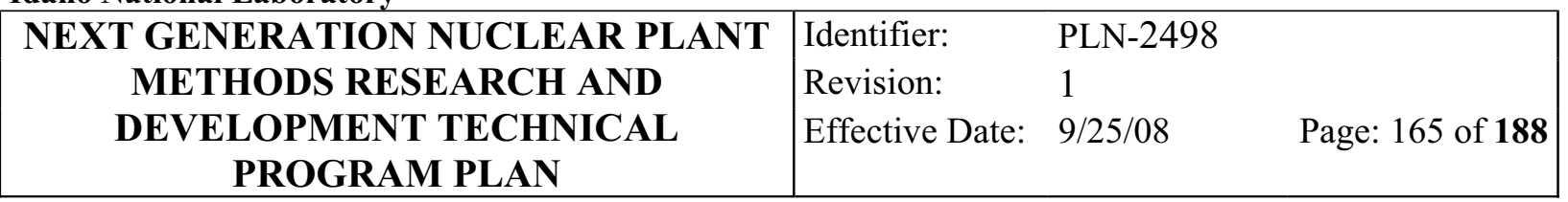

path, so that increased funding of methods R\&D should not be at the expense of "more important technical areas"; instead, increased overall funding is needed for the whole NGNP R\&D program.

\section{B-1.2 Stochastic Capabilities}

Stochastic reactor physics modeling capabilities, particularly MCNP, are regarded as well established and well verified. Since MCNP uses continuous-energy cross sections and a very general geometry description, it directly addresses neutron spectrum and spatial dependence, and it is useful for verifying other codes.

Comments are made that these tools have gaps in their ability to model double heterogeneity. Presumably these comments refer to the difficulty of representing randomly arranged fuel particles and pebbles. Efforts are being made to address this issue.

It is noted that stochastic codes are still computationally inefficient, and therefore not well suited for design optimization. Furthermore, it is pointed out that such tools cannot accommodate moving fuel.

Comments are made that "tools for truly connected and integrated stochastic methods and depletion analysis are still rudimentary," and that resources should be dedicated to this problem. Presumably the comment is directed at protocols like MOCUP and MONTEBURNS. Depletion is now available within MCNPX. It is not known whether the person who made this comment is aware of this new capability.

It is asserted that stochastic tools cannot provide spatially detailed information. One wonders whether this commenter meant that they cannot do so efficiently. With MCNP, one can define as much spatial detail as one wants. However, to get meaningful tallies in small regions, very large numbers of particles must be tracked. Variance reduction techniques can reduce these numbers to manageable size, particularly if spatially detailed information is only needed in a few spatial locations.

\section{B-1.3 Lattice/Cell Tools (Preparation of Multi-group Cross Sections)}

It is noted that the existing lattice/cell codes, such as DRAGON, WIMS, COMBINE, SCALE, and CASMO, were all developed for other types of reactors. The participants support current efforts to improve existing tools for HTGR application, including the INL's COMBINE code. However, all currently available tools need application-specific improvements, and they need V\&V for graphite systems. The participants support the currently planned development and V\&V efforts.

It is asserted that cross section sets for stochastic depletion are unreliable, not V\&V'd, and lacking in detail and accuracy. The proposed action to correct this deficiency is to devote more resources to the problem, preferably through a consortium of universities.

\section{B-1.4 Whole-Core Analysis (Multi-step Design Approach)}

Deterministic diffusion codes are considered adequate for whole-core design calculations. For PBRs, the INL code PEBBED is considered an advanced code for design optimization, although, since it is still under development, it has not been V\&V'd. Additional development needs for PEBBED are identified, including leakage feedback, non-axial pebble flow, and more sophisticated treatment of depletion.

The DIF3D/REBUS code suite is considered adequate for computing core physics parameters for the prismatic VHTR, although a need is asserted for including nodal equivalence theory (surface-dependent discontinuity factors) and pin power reconstruction in the DIF3D-VARIANT nodal transport module. 


\begin{tabular}{|c|lll|}
\hline NEXT GENERATION NUCLEAR PLANT & Identifier: & PLN-2498 & \\
METHODS RESEARCH AND & Revision: & 1 & \\
DEVELOPMENT TECHNICAL & Effective Date: & $9 / 25 / 08$ & Page: 166 of 188 \\
PROGRAM PLAN & & & \\
\hline
\end{tabular}

\section{B-1.5 Whole-Core Analysis (Spatially Heterogeneous Modeling)}

For PBRs, the INL code PEBBED and the German code VSOP are identified as appropriate codes for this kind of analysis. The same development needs for PEBBED that are identified for category 4 apply to this category as well. Furthermore, improved thermohydraulic coupling is needed.

It is noted that VSOP can model the time-dependent evolution of the steady state, which was not the purpose of PEBBED (although such a capability for PEBBED is under development). VSOP can be used for the "run-in" period, but it is a "black box." If Westinghouse and PBMR are chosen to build the NGNP, they will probably use VSOP, but VSOP will require extensive V\&V with independent tools.

In this category, the issue of pebble flow mechanics is discussed. It was noted that dynamic simulation of pebble flow is possible, and such simulation efforts have been begun at INL. It is recommended that development of these models continue, and that they be validated against experiments. The participants recommended that current efforts to develop the tools identified should continue.

\section{B-1.6 Source Term/Decay Heat Determination}

Comments recommend investigation of the use of other codes (unspecified) to generate decay heat using nuclide distributions supplied by PEBBED. The decay heat predictions could be combined with the fission power profiles predicted by PEBBED to provide complete heating profiles.

Comments note that tools exist that can compute very detailed isotopic content (e.g., ORIGEN). These tools need to be validated for graphite reactor spectra and fuel burnups. Better methods are needed to integrate these tools with core-level analysis (again, is the commenter aware of the coupling available in MCNPX?).

\section{B-1.7 Coupled Neutron-Gamma Calculation}

A comment recommends that a production module is needed for integrated neutron and gamma calculations for VHTR applications, based on the approaches used for fast reactors in DIF3D. Another comment asserts a need to identify tools for shielding and dose analysis.

\section{B-1.8 Spatial Kinetics Calculation}

It is noted that a kinetics version of PEBBED is being developed. This is considered a needed development. The German code TINTE, which is being used by PBMR, is another option for spatial kinetics calculations. Data needs are identified. For the prismatic reactor, it is suggested that DIF3D-K be coupled to a systems analysis tool. In all cases, V\&V is needed.

\section{B-1.9 Integral Data for Validation}

The lack of benchmark data for the VHTR spectrum is noted. This is especially true for the prismatic reactor. Only limited data are available to distinguish the moderator temperature coefficient of reactivity from the fuel temperature coefficient. It is pointed out that there is also a lack of data for criticality safety analysis of fuel storage and handling configurations for the VHTR fuel forms. Comments express hope that the experiments at the French MASURCA facility proposed by Areva NP will provide a substantial amount of relevant data. It is recommended that formal evaluation of existing relevant data continue in the IRPhEP process in accordance with the current Methods R\&D plan. It is also recommended that new 


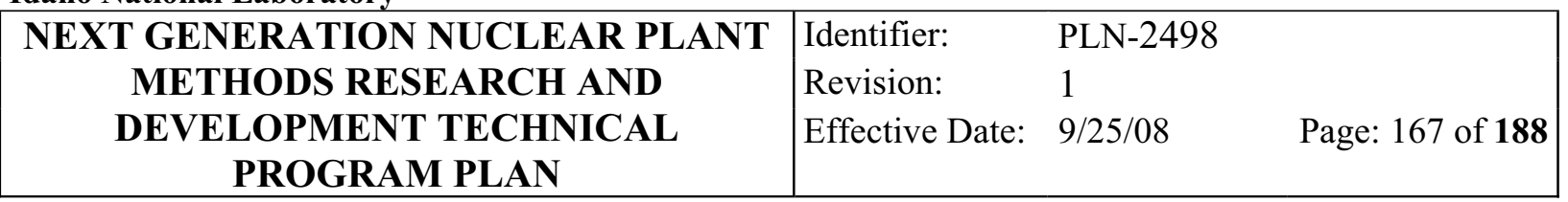

physics experiments be performed in the United States to provide specific data for VHTR methods V\&V, and that experiments be commissioned at universities that have appropriate facilities.

\section{B-1.10 Detailed Comments}

The comments can be read in their entirety by referring to Parker and West 2006.

\section{B-1.11 Summary}

Observations and comments from the Workshop participants identify appropriate tools, summarize their strengths, and list "gaps," which may be deficiencies in specific tools or the complete absence of an appropriate tool Actions to close the gaps are suggested. The comments on reactor physics tools can be divided into groups covering reactor physics parameters and pebble flow mechanics; each of these groups can be broken down according to parameters to be evaluated. For each parameter, a list of tool categories is considered. Since the comments on the tool categories are mostly the same for all parameters, the condensed comments are collected for all parameters and listed by tool category.

\section{B-2. THERMAL-FLUIDS BEHAVIOR}

The software used to calculate thermal-fluids behavior are divided into three categories: severe accident, systems analysis, and computational fluid dynamics. Examples of software which fits within these three categories are:

- Severe accident: MELCOR and GRSAC

- Systems analysis: RELAP5-3D

- Computational fluid dynamics: FLUENT and STAR-CD

The three thermal-hydraulics software categories are distinguished from one another by their focus and, consequently their numerical models and calculational capabilities. The differences between the three types are described in more detail below.

\section{B-2.1 Severe Accident}

Such codes, for example, MELCOR contain models aimed at calculating the behavior of a system following fuel failure - hence it has extensive models aimed at calculating fission product transport. These codes are generally very fast-running and do not require extensive computer resources.

\section{B-2.2 Systems Analysis}

Systems analysis codes, such as RELAP5-3D, were originally developed with one-dimensional models (with the viscous stress terms removed from the field equations) to calculate the fluid behavior throughout the reactor system including the balance-of-plant-principally during off-normal or during accident scenarios - but not system behavior following fuel failure. This class of software, in particular RELAP and RETRAN, are the most commonly used tools in performing licensing calculations. Not long ago, RELAP5 was used for NRC license audit calculations for the Westinghouse AP600 license submittal evaluation. The RELAP5 qualification process (described in Fletcher et al 1997) is the standard used to derive the qualification methodology for thermal-fluid behavior tools in the NGNP R\&D plan. 


\begin{tabular}{|c|lll|}
\hline NEXT GENERATION NUCLEAR PLANT & Identifier: & PLN-2498 & \\
METHODS RESEARCH AND & Revision: & 1 & \\
DEVELOPMENT TECHNICAL & Effective Date: & $9 / 25 / 08$ & Page: 168 of 188 \\
PROGRAM PLAN & & & \\
\hline
\end{tabular}

\section{B-2.3 Computational Fluid Dynamics}

These codes were developed from first principles and consequently retain all the terms in their field equations. Generally, CFD models have a very fine mesh and consequently are computationally intensive. CFD is generally used to model the fluid dynamics in large volumes such as the inlet and outlet plena of the NGNP as well as the reactor confinement volume. The fine mesh requirements of a high-fidelity CFD model prevent analysts from making CFD representations of an entire reactor system presently.

\section{B-2.4 Workshop Format}

The portion of the Workshop devoted to discussions on the thermal-hydraulic software and R\&D planning was generally directed to the above three classes of software. The NGNP Methods Plan describes a process to use: (a) CFD to calculate whether or not unacceptably large localized hot spots or thermal gradients are present, (b) systems analysis codes to calculate the global system behavior and also to provide boundary conditions to the CFD software, and (c) severe accident codes to perform scoping calculations (GRSAC) or to calculate fission product transport throughout the confinement, following fuel failure, and also to the environment. The three classes of software were examined and discussed in the context of calculating the thermal fluid behavior in the core, the inlet and outlet plena, adjacent to the vessel/core barrel walls, and in the confinement. Special attention was paid to the capabilities of these classes of software to calculate the presence of localized hot spots and the presence of excessively high thermal gradients.

\section{B-2.5 Summary of Comments and Observations during Workshop}

The comments and observations summarized below are recorded in the Workshop Proceedings (Parker and West 2006), Appendix D. The summaries are broken into sections specific to CFD software, systems analysis software, and severe accident software respectively.

\section{CFD Software}

CFD, as an analysis tool, is perceived to be capable of providing high-fidelity answers. CFD can be coupled to multi-dimensional core physics tools to provide high resolution estimates of hot spot magnitudes and locations. CFD is effective in calculating three-dimensional (3-D) turbulent, transitional and natural circulation (density-gradient driven) flows since: (i) heat transfer coefficients are calculated directly and radiation heat transfer is properly modeled - including analytically-calculated view factors, (ii) a spectrum of turbulence models is available to calculate turbulent behavior, (iii) large eddy simulation filtering techniques are also available to enable the analysts to focus on specific areas of importance if the Reynolds number averaged Navier-Stokes equation (RANS) approach, using turbulence models, does not represent the physics, (iv) system-wide countercurrent density-gradient driven flowssuch as will occur during the pressurized conduction cooldown scenario and the later stages (air ingress) of the depressurized conduction cooldown scenario - can be modeled if the participating portions of the system are modeled, and (v) interactions between multiple species are modeled.

Presently inadequacies of CFD software are: (a) CFD needs to be coupled to high resolution multidimensional core physics tools ${ }^{\mathrm{s}}$ and in some cases structural mechanics codes, (b) rigorous validation

s. This requirement is needed for some cases when CFD is used in or near the core. 


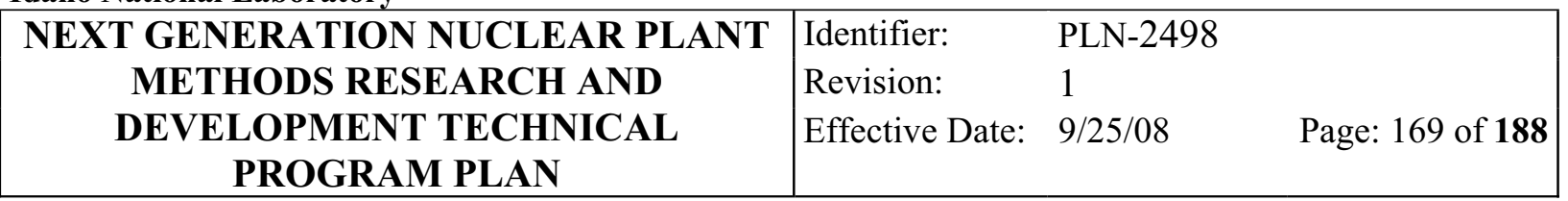

studies have not been performed since well-defined benchmark studies are not available ${ }^{t},(d)$ not defined properly since the level of validation has not been rigorously defined with respect to NRC Regulatory Guide 1.203 , (d) related to the need to treat specific geometries for which existing turbulence models may or may not be applicable, (e) not able to adequately analyze flow through porous media, (f) computationally intensive and thus CFD models are limited in size as defined by computer central processing unit limitations, and (g) Efforts to model important fluid-structure interactions are not adequate, e.g., the modeling of fluid-induced vibration or some material deformation, (h) the quality assurance requirements are not adequately formalized.

Issues and inadequacies that require resolution include:

1. Key experiments need to be defined and performed.

2. Resolution of whether separate-effects data for pebble conduction and thermophysical properties are adequate for validation given the validations are performed in conjunction with code-to-code benchmarking comparisons.

3. Resolution of the accuracies required for calculations and experiments such that the required analysis objectives are achieved given the uncertainties in graphite data.

4. The need to evaluate the experimental data, presently available, to determine whether they are of sufficient accuracy.

5. Identification of the level of detail required in the planned CFD validations and the analyses that are to follow.

6. Definition of the required fidelity of the calculated parameters needs to ensure the proposed calculations are adequate.

7. Definition of practices and procedures to enable the mesh size and model size requirements to be defined. Completion of this task will ensure the potential incompatibilities between the computational needs and the available computing power are identified and resolved.

8. Assurance that the experimental data sets and validation practices are in conformance with 10CFR 50 B, NQA-1 and 10CFR 20 reporting requirements.

9. There are presently no means to produce the required validation data that include all of the integral effects and interactions. An integral scaled facility is required to produce applicable CFD validation data.

10. The specification of practices and procedures to ensure that CFD validation data sets are adequate to not only determine whether the software are capable of calculating the required phenomena but also give sufficient information so enable software developers to determine the cause of the deficiencies. This is particularly true for large scale facilities such as the proposed integral scaled facility as well as the RCCS scaled facility.

There are presently no means to produce the required validation data that include all of the integral effects and interactions. An integral scaled facility is required to produce applicable CFD validation data.

The specification of practices and procedures to ensure that CFD validation data sets are adequate to not only determine whether the software are capable of calculating the required phenomena but also give

t. For example, appropriate turbulence models need validation for buoyant flow conditions, mixed flows, transitional flows, and high-aspect-ratio natural convection systems - such as RCCS. 


\begin{tabular}{|c|lll|}
\hline NEXT GENERATION NUCLEAR PLANT & Identifier: & PLN-2498 \\
METHODS RESEARCH AND & Revision: & 1 & \\
DEVELOPMENT TECHNICAL & Effective Date: & $9 / 25 / 08$ & Page: 170 of 188 \\
PROGRAM PLAN & & & \\
\hline
\end{tabular}

sufficient information so enable software developers to determine the cause of the deficiencies. This is particularly true for large scale facilities such as the proposed integral scaled facility as well as the RCCS scaled facility.

Summary - CFD Software: CFD software can be used to analyze the fluid dynamics in any portion of the NGNP. The largest impediment to using CFD is the computational requirements and the size of the problem that requires analysis.

Presently all of the "gaps" and "issues and inadequacies" identified by the Workshop participants are being addressed either directly by formulating the practices and procedures that are to be used for CFD V\&V or are planned to be implemented at some stage in the NGNP Design and Safety Methods Validation Program.

\section{Systems Analysis Software}

Systems analysis software, such as RELAP5, are ideal tools to analyze the behavior of system components that can be represented by a one-dimensional analysis. Some systems analysis codes, although they have limited 3-D modeling capability, are usually missing some terms in the field equations, e.g., the viscous stresses. Consequently, without being prescriptive, an analyst cannot model such phenomena as turbulent mixing properly. However, systems analysis software, due its limited computational needs, can easily be used to model the entire system, including the balance-of-plant over the full extent of the transient scenario. Hence, first-order solutions to the behavior in all of the system components can be obtained. And, if the systems analysis code is used in conjunction with a CFD code, the systems analysis code can be used to provide the boundary conditions to the CFD calculation. In this way a systems analysis code can be used to provide the boundary conditions for the CFD code for longterm transients. That is, if it is desired to study the mixing in the inlet and outlet plena some time after an accident scenario is initiated, e.g., at time equal 24 hours, the systems analysis code can be used to obtain the boundary conditions for the CFD code at that desired time - and with this input the CFD code can then be used to describe the mixing and density-gradient driven flow behavior at selected times in the scenario.

A special use of systems analysis codes is to analyze the behavior of two-phase problems in the NGNP. Two-phase problems will be likely to occur in the water-cooled RCCS. Since, under natural circulation conditions, the water is very likely to boil and thus two-phase flow regimes will be present, the systems analysis codes are the only available tool with the capability to address this problem properly.

Presently inadequacies of systems analysis software are: (a) heat transfer correlations for transition and mixed convection need to be developed and implemented, (b) rigorous validation studies have not been performed based on the highly-ranked phenomena identified in the phenomena identification and ranking studies, (c) not defined properly since the level of validation has not been rigorously defined with respect to NRC Regulatory Guide 1.203, (d) related to the need to treat specific geometries and heat transfer loads - such as might occur in the core, e.g., possible heat transfer in channels with high heat loads on one wall and low heat loads on an adjacent wall, and (e) the quality assurance requirements are not adequately formalized.

Issues and inadequacies that require resolution include:

1. Key experiments need to be defined and performed. 


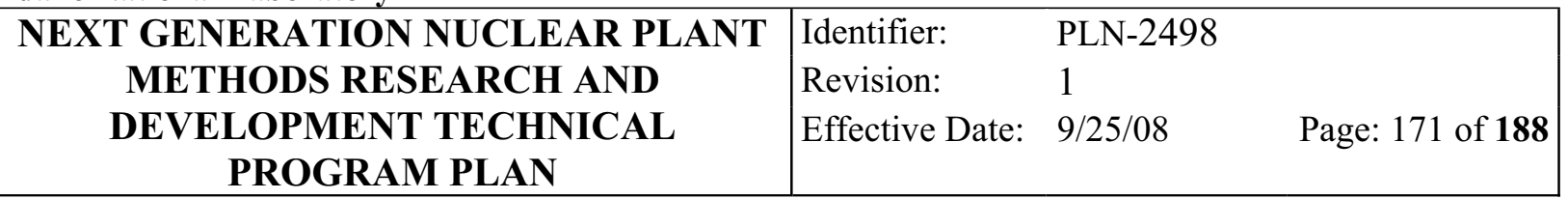

2. Resolution of whether separate-effects data for pebble conduction and thermophysical properties are adequate for validation given the validations are performed in conjunction with code-to-code benchmarking comparisons.

3. Resolution of the accuracies required for calculations and experiments such that the required analysis objectives are achieved given the uncertainties in graphite data.

4. The need to evaluate the experimental data, presently available, to determine whether they are of sufficient accuracy.

5. Identification of the level of detail required in the planned systems analysis software validations and the analyses that are to follow.

6. Definition of the required fidelity of the calculated parameters needs to ensure the proposed calculations are adequate.

7. Definition of practices and procedures to enable the nodalization and model size requirements to be defined.

8. Assurance that the experimental data sets and validation practices are in conformance with $10 \mathrm{CFR} 50$ B, NQA-1 and 10CFR 20 reporting requirements.

9. There are presently no means to produce the required validation data that include all of the integral effects and interactions. An integral scaled facility is required to produce applicable systems analysis software validation data.

10. The specification of practices and procedures to ensure that systems analysis software validation data sets are adequate to not only determine whether the software are capable of calculating the required phenomena but also give sufficient information so enable software developers to determine the cause of the deficiencies. This is particularly true for large scale facilities such as the proposed integral scaled facility as well as the RCCS scaled facility.

Summary - Systems Analysis Software: Systems analysis software can be used to analyze the fluid dynamics in any portion of the NGNP. Presently all of the "gaps" and "issues and inadequacies" identified by the Workshop participants are being addressed either directly by formulating the practices and procedures that are to be used for systems analysis V\&V or are planned to be implemented at some stage in the NGNP Design and Safety Methods Validation Program.

\section{Severe Accident Software}

The two severe accident analysis software tools considered by the Workshop participants were MELCOR and GRSAC. GRSAC is a very fast-running code that is ideal for performing scoping calculations that describe not only core temperature distributions and other important temperature distributions (e.g., vessel temperatures and confinement material temperatures) as a function of time, but also performs graphite oxidation calculations due to air ingress. MELCOR is also a fast-running code that will be used to calculation fission product transport from the vessel into the reactor confinement.

The Workshop participants did not focus as intensely on these tools as the CFD and systems analysis tools. However, in general the same general comments were given for these tools as for CFD and systems analysis tools, that is, items 1 through 10 under the systems analysis section are also true for GRSAC and items 4 through 10 are applicable to MELCOR. 


\begin{tabular}{|c|lll|}
\hline NEXT GENERATION NUCLEAR PLANT & Identifier: & PLN-2498 & \\
METHODS RESEARCH AND & Revision: & 1 & \\
DEVELOPMENT TECHNICAL & Effective Date: & $9 / 25 / 08$ & Page: 172 of 188 \\
PROGRAM PLAN & & & \\
\hline
\end{tabular}

Summary - Severe Accident Software: Presently all of the "gaps" and "issues and inadequacies" identified by Workshop participants are being addressed in much the same way as those for systems analysis codes. 


\begin{tabular}{|c|lll|}
\hline NEXT GENERATION NUCLEAR PLANT & Identifier: & PLN-2498 \\
METHODS RESEARCH AND & Revision: & 1 & \\
DEVELOPMENT TECHNICAL & Effective Date: & $9 / 25 / 08$ & Page: 173 of 188 \\
PROGRAM PLAN & & & \\
\hline
\end{tabular}

\section{Appendix C}

\section{Differential Nuclear Data Measurements at the ANL Intense Pulsed Neutron Source}


NEXT GENERATION NUCLEAR PLANT

METHODS RESEARCH AND

DEVELOPMENT TECHNICAL

PROGRAM PLAN
Identifier: $\quad$ PLN-2498

Revision: $\quad 1$

Effective Date: 9/25/08 Page: 174 of 188 


\begin{tabular}{|c|lll|}
\hline NEXT GENERATION NUCLEAR PLANT & Identifier: & PLN-2498 & \\
METHODS RESEARCH AND & Revision: & 1 & \\
DEVELOPMENT TECHNICAL & Effective Date: & $9 / 25 / 08$ & Page: 175 of 188 \\
PROGRAM PLAN & & & \\
\hline
\end{tabular}

\section{Appendix C}

\section{Differential Nuclear Data Measurements at the ANL Intense Pulsed Neutron Source}

The Idaho National Laboratory (INL) collaboration with Argonne National Laboratory (ANL) involves use of sophisticated detector arrays, supporting electronics, and data acquisition systems originally established by INL on a beamline of the Argonne Intense Pulsed Neutron Source (IPNS) facility for use in fundamental studies of the fission process. The INL apparatus, which consists of an array of multiple types of multiple detectors operated in coincidence, with correlation of each observed event to the time of flight of the neutron that induced it, also offers an innovative method for cross-section measurements. The immediate measurement goals involve measurement of neutron induced interaction cross-sections for ${ }^{240} \mathrm{Pu},{ }^{242} \mathrm{Pu}$, and possibly ${ }^{241} \mathrm{Am}$, with measurements for other nuclides of interest for advanced reactor physics applications (e.g., ${ }^{241} \mathrm{Pu}$ ) to follow later.

The ANL IPNS facility is a spallation neutron source with a moderated neutron beam that has a neutron spectrum at 12 and 20 meters as illustrated in Figure C-1. The data shown in Figure C-1 are the results of average measured intensities and Monte Carlo N-Particle Transport Code (MCNP) calculations performed at IPNS. For perspective, a direct comparison of flux intensity is shown between IPNS and the Los Alamos Lujan Center at lower neutron energies where explicit numbers, in similar units, are available, in particular for epithermal neutrons.

The uncertainty of the energy of a neutron that induces an interaction of interest in the target is determined largely by the uncertainty on the n-TOF from the time width of the proton pulse. In addition to this important parameter, the pulse rate and the flight path length interact to limit the useable energy range of the neutrons. At IPNS the pulse rate is $30 \mathrm{~Hz}$ and the proton pulse full width is $70 \mathrm{~ns}$. All beam lines at IPNS are heavily shielded and evacuated so that backgrounds are reduced. The low background at IPNS is an important factor for the long runs needed for experiments that achieve low statistical uncertainty. 


\begin{tabular}{|c|lll|}
\hline NEXT GENERATION NUCLEAR PLANT & Identifier: & PLN-2498 & \\
METHODS RESEARCH AND & Revision: & 1 & \\
DEVELOPMENT TECHNICAL & Effective Date: & $9 / 25 / 08$ & Page: 176 of 188 \\
PROGRAM PLAN & & & \\
\hline
\end{tabular}

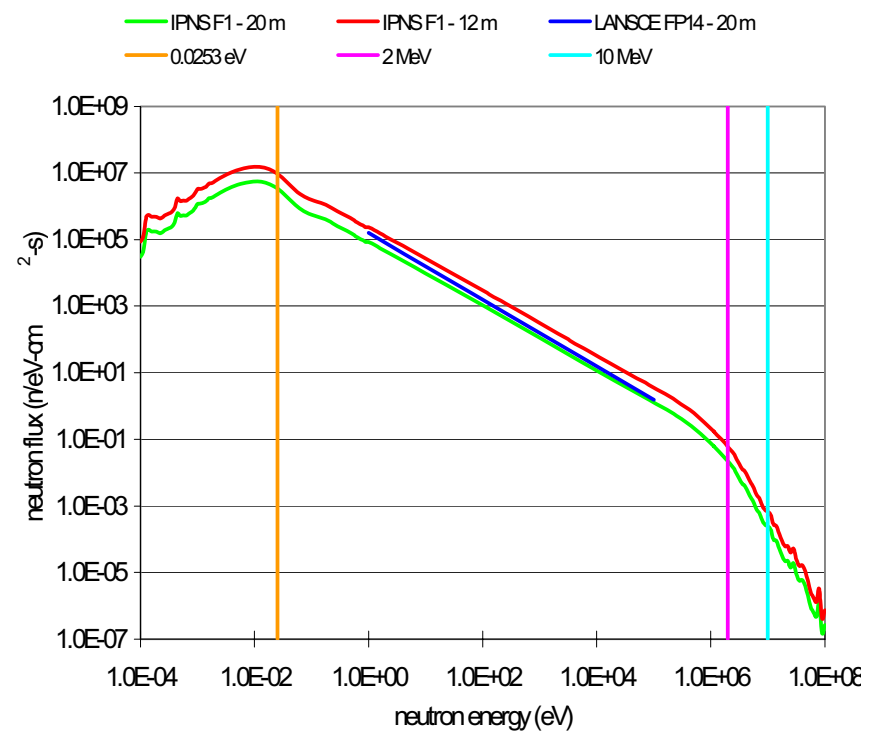

Figure C-1. Plot of the neutron spectrum on the F3 beam line used for the INL apparatus for distances of 12 and 20 meters and the extrapolated values for FP14 at LANSCE. Energy markers are shown for the readers benefit.

The INL apparatus was originally installed at IPNS to perform experiments using induced fission of actinide targets for prompt information concerning fission yields by isotope pairs, nuclear structure information for prompt de-excitation of the fission products, multiplicity of both neutron and gamma rays by isotope pairs, and isotope pair distributions for fission cluster models. These efforts are extensions of spontaneous fission studies on ${ }^{252} \mathrm{Cf}$ and ${ }^{242} \mathrm{Pu}$ conducted with arrays of HPGe detectors at INL, Oak Ridge National Laboratory (ORNL), Lawrence Berkeley National Laboratory (LBNL), and finally on GAMMASPHERE at both ANL and LBNL. This work has produced publications on the nuclear structure of fission products prior to beta decay, fission yields by isotope pairs, and explicit neutron multiplicity as correlated to specific fission pairs.

Three years ago efforts began to modify the INL apparatus at IPNS to provide the capability to measure neutron interaction cross-sections as a function of incident neutron energy, branching ratios for the production of different isotopes by neutron capture or fission, and cross-sections for the production of independent yields from actinide fission. The goal of the work is to produce results

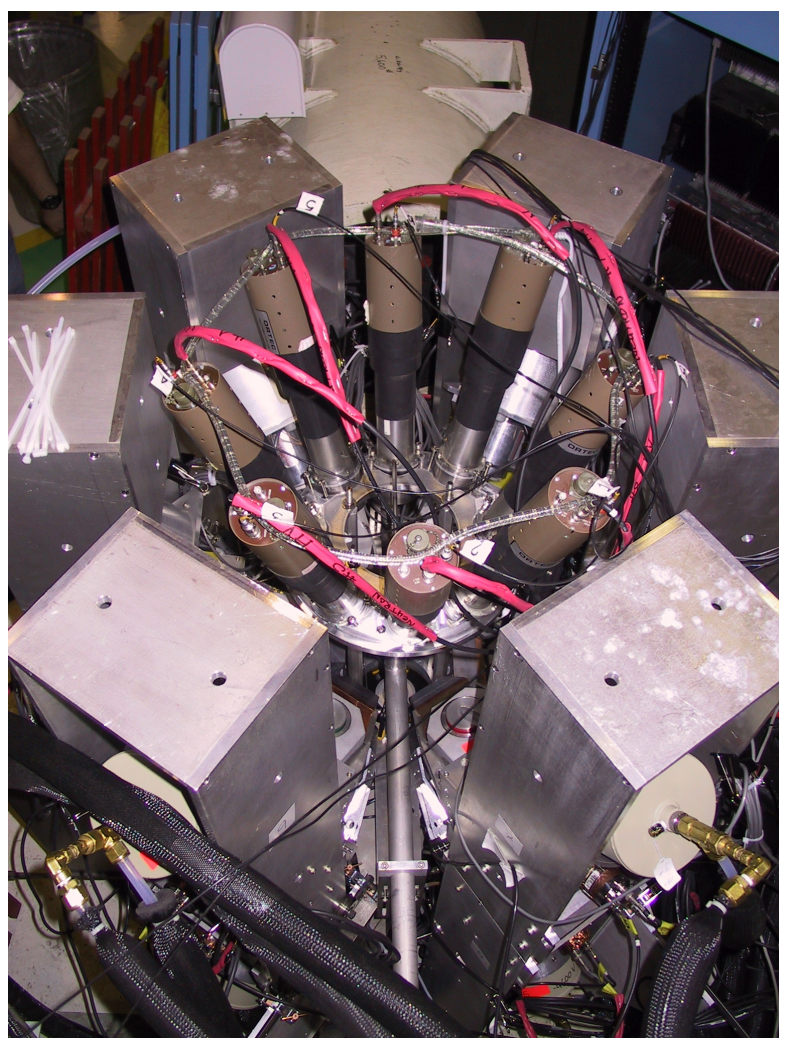

Figure C-2. INL detector array for nuclear data measurements at ANL/IPNS. 


\begin{tabular}{|c|lll|}
\hline NEXT GENERATION NUCLEAR PLANT & Identifier: & PLN-2498 \\
METHODS RESEARCH AND & Revision: & 1 & \\
DEVELOPMENT TECHNICAL & Effective Date: & $9 / 25 / 08$ & Page: 177 of 188 \\
PROGRAM PLAN & & & \\
\hline
\end{tabular}

that have statistical uncertainties of $3 \%$ or less and maximum neutron TOF uncertainties of $10 \%$. In the resonance energy range of a few $\mathrm{eV}$ to $\sim 100 \mathrm{keV}$ the TOF uncertainties are much smaller, ranging from 0.2 to $\sim 2 \%$ for both fission and capture cross-sections.

The INL experimental technique allows a model-independent measurement of the neutron interaction cross-section to be made over a continuous energy range from a few meV to above $2 \mathrm{MeV}$ without breaking the measurement into different energy segments. With approximately 4,000 hours of beam time available for measurements in one year, low statistical uncertainty can be achieved.

The INL detector array, shown in Figure C-2, is composed of 12 Compton suppressed, high purity germanium (CSHPGe) detectors, eight fast neutron detectors (BC501 liquid scintillator), and a stack of up to 32 Silicon (Si) detectors interleaved with double-sided foils of actinide targets. The trigger electronics starts the digitization process if two of the CSHPGe, two neutron detectors, or a CSHPGe and a neutron detector produce a signal within a set coincidence time window. In this way, three separate conditions can be used as independent triggers for determining if a neutron interaction has occurred in a target. The coincidence is based on overlap timing with a time window of 50 to 100 nanoseconds.

The Si detectors are used to directly detect the fission fragments as these fragments recoil directly into the Si detectors. The Si detectors and the target foils are interleaved, each actinide foil has a selected thickness that will allow the low energy, light mass fission fragment to escape the target and enter the $\mathrm{Si}$ detector, which is in contact with the target material. A discriminator is used to reject $\alpha$-particles and their pileup signal and accept only the fission fragment signal, which is a factor of ten greater in amplitude. These fission fragment signals in the Si detectors are used as a fourth trigger in the system. Si detectors are used instead of a fission chamber primarily because the rise time of the output pulse is faster by roughly a factor of ten. In addition, since the Si detector is in contact with the actinide target, gamma rays observed from the fragment have no Doppler shift or broadening due to emission in flight. There are other advantages with size, less support electronics, better $\alpha$-particle discrimination, and low mass material. The energy output of each $\mathrm{Si}$ detector is also digitized and included in the data packet.

Signals from the four triggers are combined logically in various ways to produce an event signal. This event signal is used to trigger data acquisition of digitized detector signals, time relationships between the detector signals, and as a stop on a multistop time-digital converter (TDC). In this way a multiparameter data packet is acquired for each observed radiation event and stored in list mode format. Since the system is configured to respond to coincidence events, and the prompt timing of an event is from 10-22 seconds to 10-12 seconds, a single trigger can result in multiple radiation types being included in the event. The simplest example is that of a fission fragment being detected in a Si detector and single gamma ray or neutron radiation also observed as will be shown later.

With the ability to select the events by post experiment sorting the multiplicities of both neutrons and gamma rays can be determined. The production rates of the selected gamma rays arising from the prompt fission fragments or the excited isotopes produced by neutron capture are used to determine the appropriate reaction cross-sections as a function of the incident neutron energy, determined from the event-by-event neutron TOF parameter. In addition to information required to extract absolute reaction cross-sections, the acquired data sets also will contain the information needed to extract independent fission fragment yields that can be used to validate accepted values in a model independent manner.

The most important aspect of this powerful ability to select events by sorting and then determine cross-sections is to reduce background and events unassociated with the reaction channel of interest. This reduces the error on the cross-section by allowing an event set to be selected that only contains events that are from that particular reaction channel. For example, problems associated with measuring the fission 


\begin{tabular}{|c|lll|}
\hline NEXT GENERATION NUCLEAR PLANT & Identifier: & PLN-2498 & \\
METHODS RESEARCH AND & Revision: & 1 & \\
DEVELOPMENT TECHNICAL & Effective Date: & $9 / 25 / 08$ & Page: 178 of 188 \\
PROGRAM PLAN & & & \\
\hline
\end{tabular}

cross-section for material with high spontaneous fission rates can be handled by sorting events based on different conditions. This method also reduces the total error whereas the traditional method of beam-on, beam-off does not remove the spontaneous fission events from the beam on data set. Selecting fission events by requiring a Si detector signal, and then sorting on gamma rays from different fission pairs will provide information on contributions for the two types of fission processes. The distribution of neutrons and thus associated fragments pairs are different for the spontaneous versus induced fission. This is caused by the differences in the excitation energy of the fissioning nucleus. In spontaneous fission, the nucleus is in its ground state. For induced fission, the nucleus will be at an excited state due to the energy brought in by the incident neutron and by the rearrangement of the population of the nuclear orbitals in the nuclear system after the neutron is captured.

The INL apparatus at IPNS thus has some key unique capabilities, supported by the IPNS facility itself. The most important is the ability to take coincidence data associated with a particular nuclear event as noted above. An array of detectors could be operated in this manner at other facilities as well, but at IPNS, two important enabling features are available: an intense flux of neutrons, and the availability of the beam for long experimental measurements. These two facts allow low statistical uncertainties to be attained. Achieving a goal of $\sim 109$ events observed and stored by the data system requires over 100 days of beam time. This long experimental time is available at IPNS since the INL apparatus is on one beam line and not affecting experiments at other locations in the facility. The other unique capability associated with the INL protocol is related to the ability to perform nuclear-event-based data collection and post analysis by sorting data into subsets based on physics conditions. By imposing multiple conditions and sorts via software or computer processing in selecting data sets for detailed analysis, the "cross talk" between channels can be minimized in ways that are not possible in the hardware of the electronics. The simplest and easiest to understand is the Si detector trigger to separate fission events and all other events. The nonfission events can be further sorted to look at other reaction channels. This capability has produced exceptional results in nuclear structure and spontaneous fission studies and also applies to the problems of measuring various neutron cross-sections of actinide isotopes.

The post analysis capability based on data selection of reaction channels provides results that are selfconsistent across the larger experimental data set. This means that the ability to use different approaches in sorting can produce results that provide consistency checks that are otherwise not available. An example of this is the case of the determination of a fission cross-section by direct selection of the observation from the Si detectors and the cross-section determined from sorting on gamma rays from the highest yield fission fragments. Although the statistics in these two subsets of data will be different, the cross-section should have the same result in both cases. This is a powerful tool to check the results and provide a consistency not in previous work.

Targets used by INL at the IPN facility are fabricated in Russia by collaborators at the Joint Institute for Nuclear Research (JINR). The targets are metal foils, not oxides, on an appropriate backing and of the thickness needed to allow the light fission fragments to escape the target and enter the silicon detector that is in contact with the target material. This removes the need for large corrections of the incident neutron flux that must often be done in the case of oxide targets. In addition, the vapor deposition of metal onto metal backing gives excellent stability to the targets and reduces the risk of contamination due to targets coming off the backing. The isotopic purity of the targets is greater than $98 \%$ for the principal isotope, and a detailed chemical analysis is provided for each target batch and individual target characteristics such as mass per unit area, total mass, and others.

Authorization was received in January 2005 for internal INL funding to perform a proof-of-principle experiment for cross-section measurements at IPNS using ${ }^{239} \mathrm{Pu}$ standards. This experiment was 


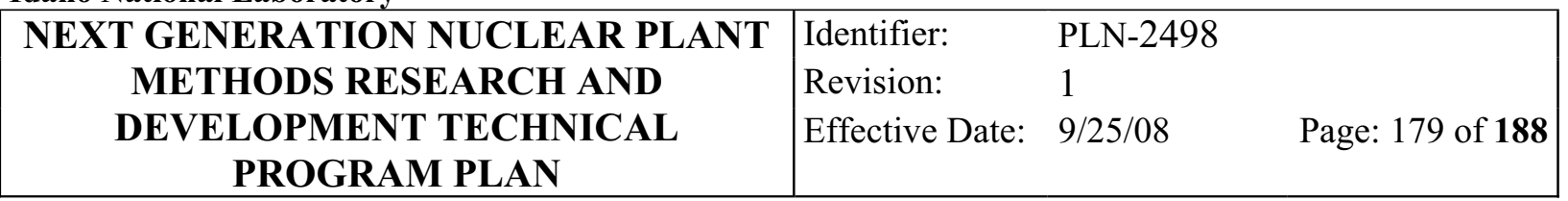

recommended in a September 2004 international peer review of the INL-ANL proposal to perform some types of nuclear data measurements pertinent to the VHTR Generation-4 program at IPNS. The experiment was successfully initiated in early May 2005 and data collection for the most recent accelerator operation cycle continued until the end of the cycle in late June 2005. Significant results were obtained and presented at the DOE AFCI/Generation IV Physics Working Group meeting in July 2005. Some key initial results are summarized below.

Figure C-3 shows some initial time-of-flight (TOF) correlated fission event spectrum data from the INL silicon detectors over the energy range of interest from thermal to about $1 \mathrm{MeV}$. The prominent first resonance in plutonium at $0.3 \mathrm{eV}$ is labeled, as are a few of the higher resonances. Recall that as one goes to the left in this plot the incident neutron energy increases to first order as the inverse square of the time of flight. Figure C-4 shows the same data with the energy range up to about $500 \mathrm{eV}$ expanded to show more detail of the measured data for the prominent resonances.

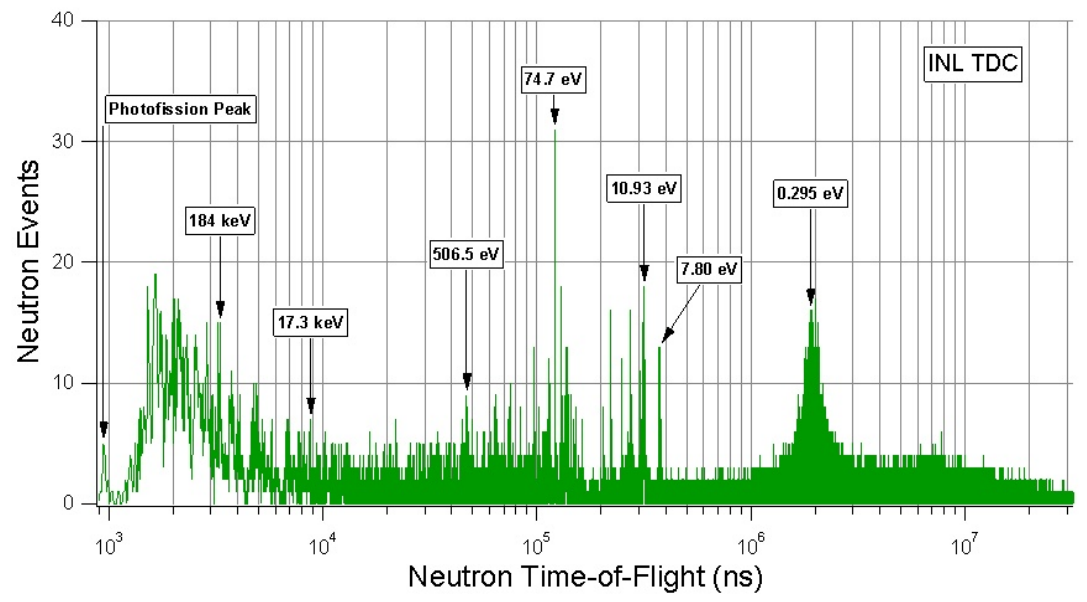

Figure C-3. Initial 239Pu Fission event spectrum from the IPNS nuclear data proof of principal experiment at ANL/IPNS. The neutron energy range spans 8 decades.

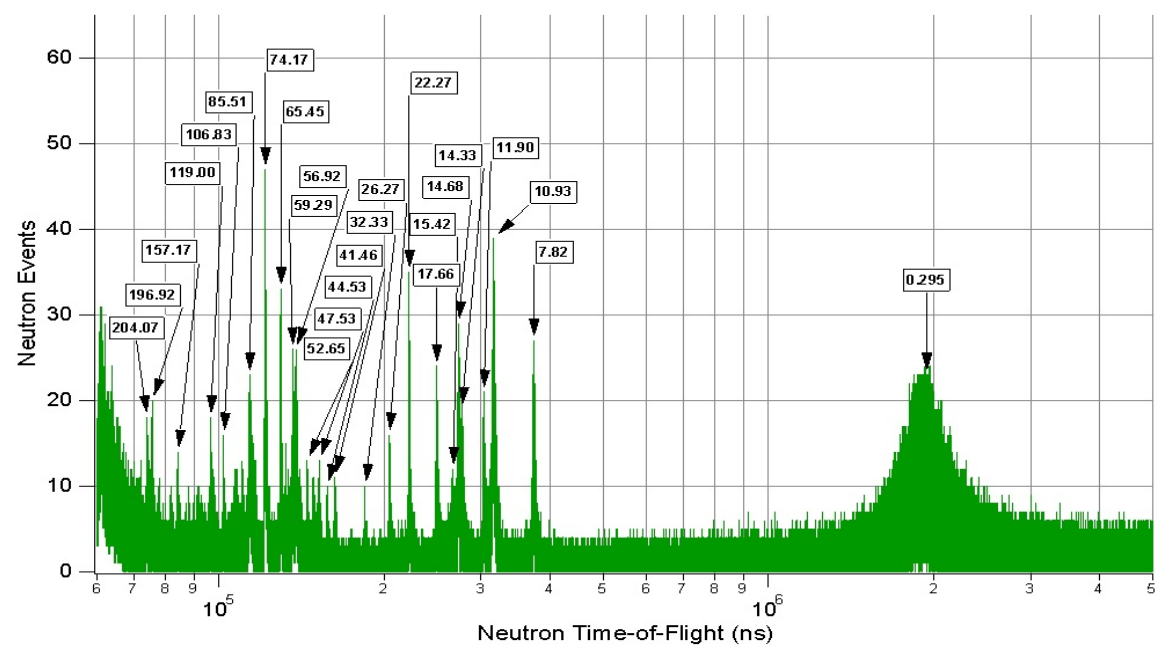

Figure C-4. Fission event spectrum for ${ }^{239} \mathrm{Pu}$. Expanded energy scale. 


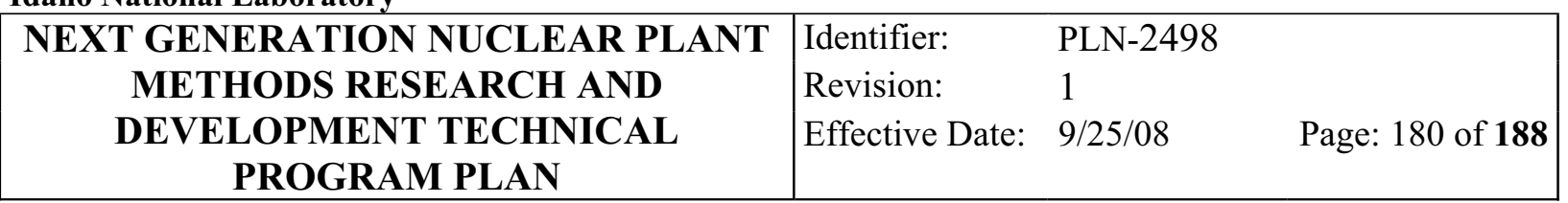

Figure C-5 shows a further expansion of the energy scale to show detail in the energy range around $200 \mathrm{eV}$. The measured data are given in the lower plot, while the ENDF evaluation of the corresponding cross-section for the same energy range is given in the top plot for comparison. Note the remarkable fidelity of the measured data relative to the standard evaluation. The statistical uncertainty on the measurements in these preliminary results is on the order of $10 \%$ at the resolution shown. However, these early demonstration experiments only involve collection of data over a few days of run time. In a precision calibration, or for a measurement of the cross-section for an unknown sample, the run time would be much longer, to permit greater statistical accuracy on the order of the requirements identified by the various uncertainty propagation studies.
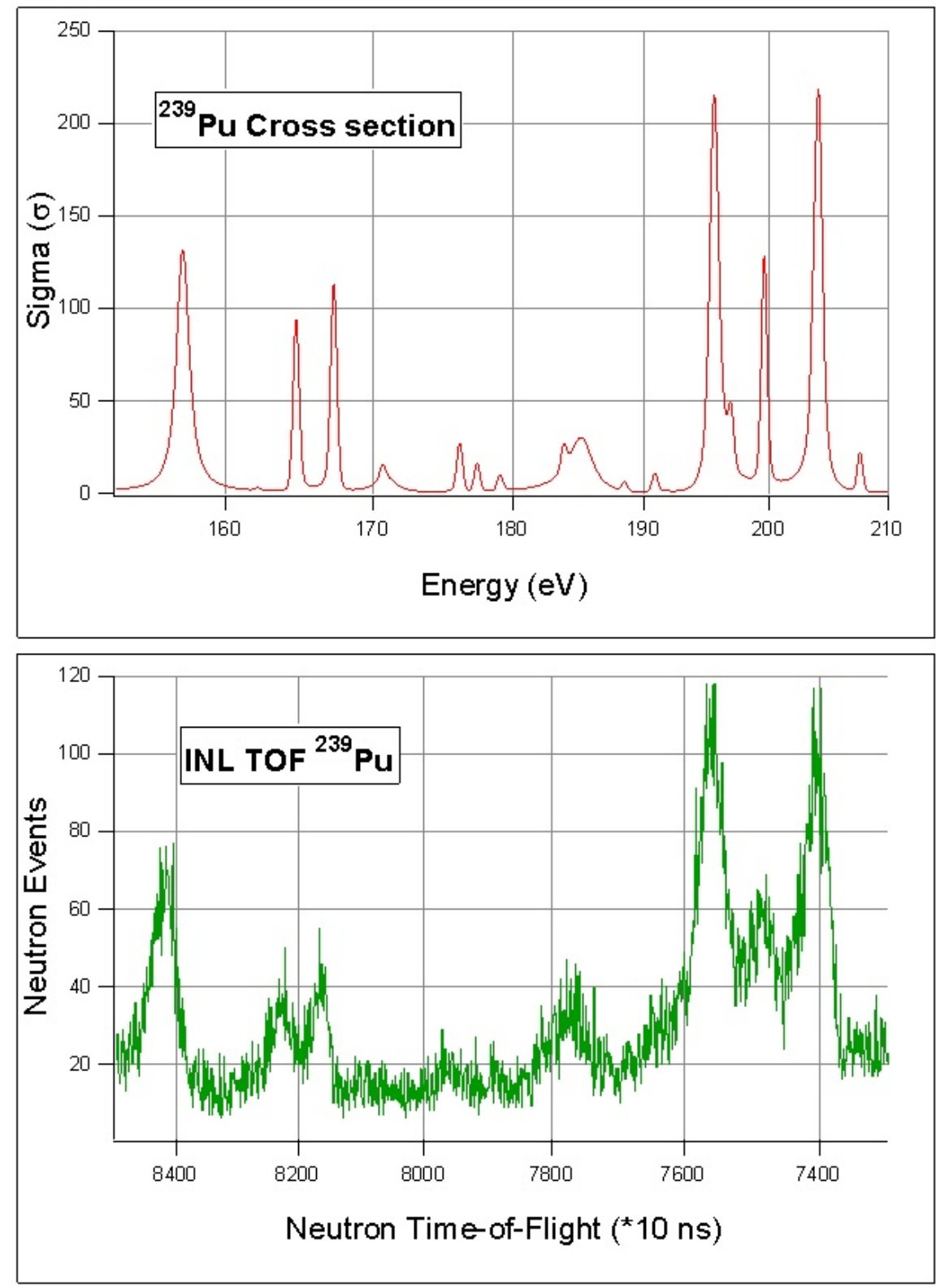

Figure C-5. Comparison of the ENDF fission cross-section (top) with the INL-measured fission event spectrum (bottom). 


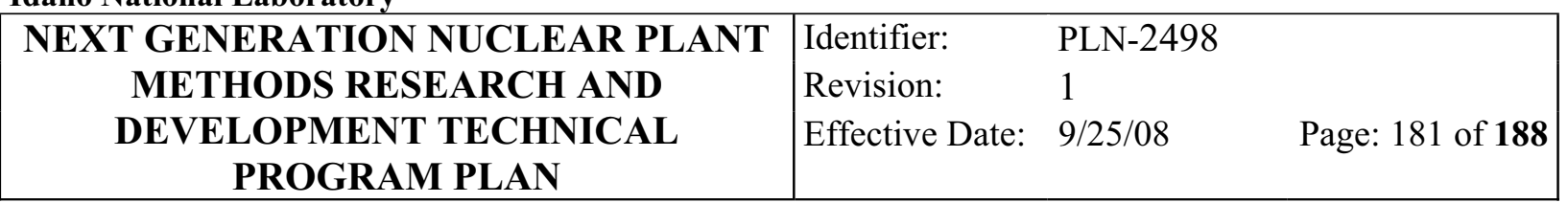

Finally, and most significantly, it should be noted that the results given in the previous figures are for "singles" data - data from the silicon fission detectors alone, with no coincidence gating. Figure C-6 provides a striking initial example of what can be achieved by coincidence gating. In this case, the signals from the gamma detectors in the INL/IPNS detector array are gated (by postexperiment sorting) in anticoincidence with the signals from the silicon fission detectors. This offers a self-consistent way of separating neutron capture events from fission events in a way that avoids the need to do individual experiments for each, or to normalize data to computational models. That is, an event detected by the gamma detectors is only counted if a fission is NOT detected at the same time. Figure C- 6 shows the results of this procedure for the energy range around $10 \mathrm{eV}$. The top plot once again shows the ENDF cross-section (the standard for comparison) and the bottom plot shows the gated data or neutron capture event spectrum (proportional to the capture cross-section), separated from the fission event spectrum solely by experiment, in the same experiment, a key feature of the coincidence approach used at IPNS.
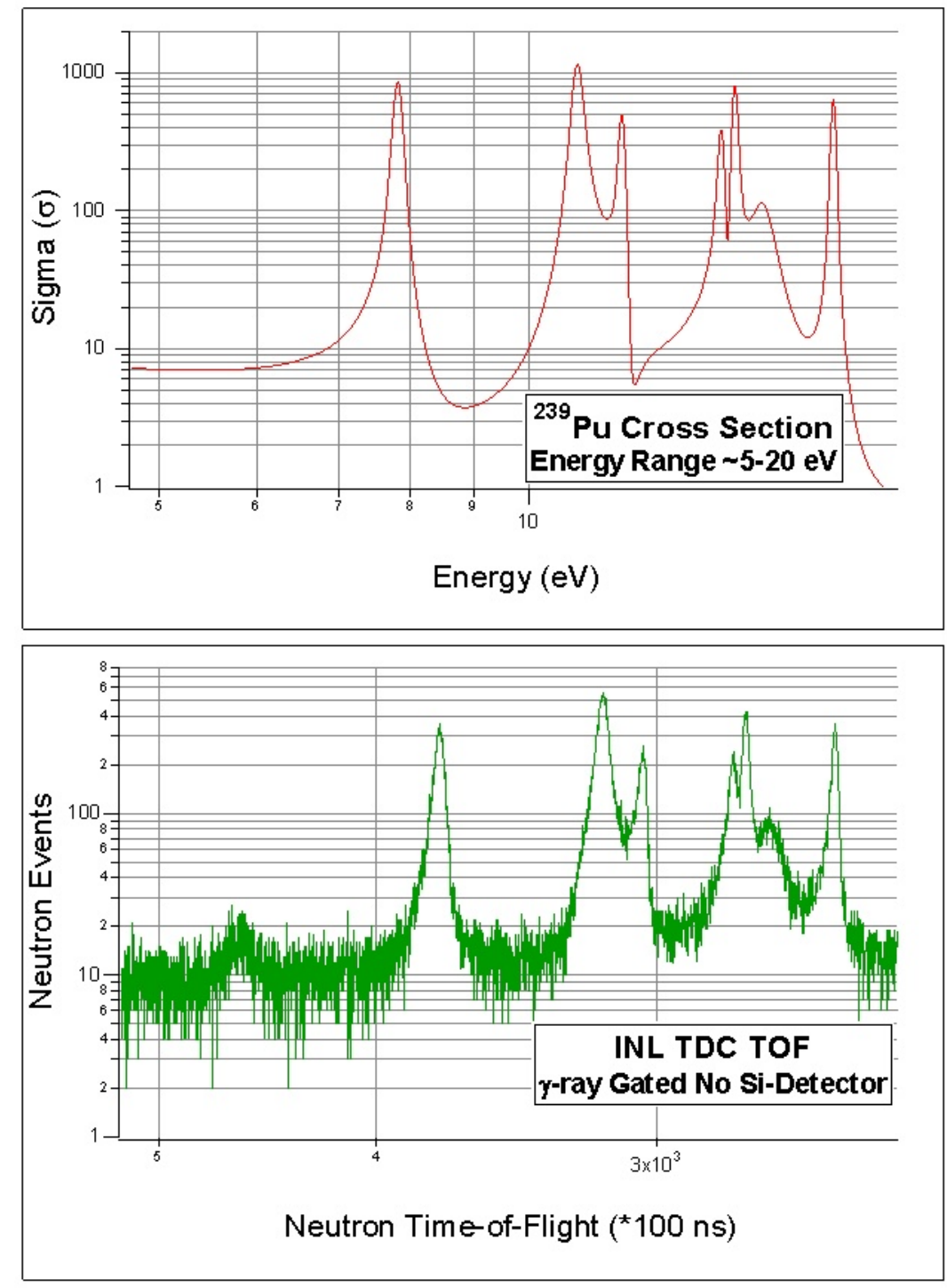

Figure C-6. Capture event spectrum (bottom) for ${ }^{239} \mathrm{Pu}$ measured by INL using anticoincidence with fission, compared with the ENDF capture cross-section evaluation (top). 


\begin{tabular}{|c|lll|}
\hline NEXT GENERATION NUCLEAR PLANT & Identifier: & PLN-2498 & \\
METHODS RESEARCH AND & Revision: & 1 & \\
DEVELOPMENT TECHNICAL & Effective Date: & $9 / 25 / 08$ & Page: 182 of 188 \\
PROGRAM PLAN & & & \\
\hline
\end{tabular}

Once again the high fidelity of the measured data apparent, even though the initial results are for only a few days of accelerator run time. Of particular interest is the small feature that appears in the measured data near $5 \mathrm{eV}$. This small resonance is not included in the ENDF evaluation, nor is it included in the commonly used JENDL or JEFF evaluations (source: NEA Janis 2.1). It is, however, reported in the Russian BROND data evaluation, and the new data from the IPNS experiment reported here independently confirms it. In fact, a detailed examination of the data offers intriguing evidence that this resonance and a few others that were observed could be primarily related to previously unrecognized channels for induced alpha emission.

The coincidence approach also allows many other types of signals to be separated. For example, separating a neutron-induced event spectrum (cross-section) from the very high background radioactivity present in some actinide targets of interest can be very effectively enabled by this technique.

Compensation for unwanted background due to target contamination is also possible. Finally, and very significantly, it may also be possible to resolve resonances in a manner that is not limited by the proton pulse width of the accelerator by capturing, through coincidence techniques, differences that may exist between the decay schemes of each resonance, although this remains to be demonstrated. Taken together, these features of the experimental apparatus and protocol offer the potential for improved accuracy in differential actinide cross-section measurements important to VHTR as well as to other Generation IV concepts.

\section{Workscope for FY 2007}

- Per recommendations of the DOE Joint AFC/Generation IV Physics Working Group, INL, in collaboration with ANL, will perform the following two tasks in FY 2007:

- Complete a demonstration measurement of Pu-239 absolute fission and capture cross-section as a function of energy over an energy range from thermal up to the energy resolution limit of the experimental apparatus currently operating at the ANL IPNS facility. Present available results orally at upcoming GNEP/AFC/Generation IV Physics Working Group Meetings and issue a written external INL report of all results by September 30, 2007. Results will also be submitted for publication in the refereed literature as appropriate.

- In the first quarter of FY 2007, specify and order long-lead-time, high isotopic purity targets needed for completion of the ${ }^{239} \mathrm{Pu}$ measurements in FY 2007 and initiation of planned measurements of cross-sections for ${ }^{240} \mathrm{Pu}$ in FY 2008. This task will also include specification of ${ }^{242} \mathrm{Pu}$ targets for initiation of measurements for that isotope after FY 2008 as well due to economies that can be achieved by ordering both target sets together.

- Deliverables for FY 2007 will include monthly status reports of the experiment, the equipment, and operational results, oral report to the Physics Working Group as noted, an Annual Report, and any papers presented or accepted for publication.

- $\quad$ Estimated costs for FY 2007 are \$1,200K.

\section{Workscope for FY 2008}

- Complete documentation of ${ }^{239} \mathrm{Pu}$ calibration and demonstration. Initiate induced interaction measurements for ${ }^{240} \mathrm{Pu}$. Initiate spontaneous fission measurements for ${ }^{240} \mathrm{Pu}$ using Gammasphere at ANL for various corrections, calibrations, and other basic information to facilitate the IPNS measurements. 


\begin{tabular}{|c|lll|}
\hline NEXT GENERATION NUCLEAR PLANT & Identifier: & PLN-2498 \\
METHODS RESEARCH AND & Revision: & 1 & \\
DEVELOPMENT TECHNICAL & Effective Date: & $9 / 25 / 08$ & Page: 183 of 188 \\
PROGRAM PLAN & & & \\
\hline
\end{tabular}

- Deliverables for FY 2008 will include monthly status reports of the experiment, the equipment, and operational results, periodic oral reports to the Physics Working Group, an Annual Report, and any papers presented or accepted for publication.

- Estimated costs for FY 2008 are $\$ 2,000 \mathrm{~K}$.

\section{Workscope for FY 2009}

- Complete ${ }^{240} \mathrm{Pu}$ measurements and initiate ${ }^{242} \mathrm{Pu}$ measurements.

- Deliverables for FY 2009 will include monthly status reports of the experiment, the equipment, and operational results, periodic oral reports to the Physics Working Group, an Annual Report, and any papers presented or accepted for publication.

- Estimated costs for FY 2009 are $\$ 2,000 \mathrm{~K}$.

\section{Workscope for FY 2010}

- Complete ${ }^{242} \mathrm{Pu}$ data measurements and data analysis.

- Deliverables for FY 2010 will include monthly status reports of the experiment, the equipment, and operational results, periodic oral reports to the Physics Working Group, an Annual Report, and any papers presented or accepted for publication.

- Estimated costs for FY 2010 are: $\$ 2,000 \mathrm{~K}$.

\section{Workscope Beyond FY 2010}

Workscope beyond FY 2010 will be dependent on nuclide priorities set by the Generation IV/AFCI Physics Working Group, but it is anticipated that the effort will include measurements of certain americium and curium isotopes of interest for potential advanced fuel cycles. 
NEXT GENERATION NUCLEAR PLANT

METHODS RESEARCH AND

DEVELOPMENT TECHNICAL

PROGRAM PLAN
Identifier: $\quad$ PLN-2498

Revision: $\quad 1$

Effective Date: 9/25/08 Page: 184 of 188 


\begin{tabular}{|c|lll|}
\hline NEXT GENERATION NUCLEAR PLANT & Identifier: & PLN-2498 \\
METHODS RESEARCH AND & Revision: & 1 & \\
DEVELOPMENT TECHNICAL & Effective Date: & $9 / 25 / 08$ & Page: 185 of 188 \\
PROGRAM PLAN & & & \\
\hline
\end{tabular}

\section{Appendix D}

\section{The Use of Nuclear Energy for Process Heat}


NEXT GENERATION NUCLEAR PLANT

METHODS RESEARCH AND

DEVELOPMENT TECHNICAL

PROGRAM PLAN
Identifier: $\quad$ PLN-2498

Revision: $\quad 1$

Effective Date: 9/25/08 Page: 186 of $\mathbf{1 8 8}$ 


\begin{tabular}{|c|lll|}
\hline NEXT GENERATION NUCLEAR PLANT & Identifier: & PLN-2498 \\
METHODS RESEARCH AND & Revision: & 1 & \\
DEVELOPMENT TECHNICAL & Effective Date: & $9 / 25 / 08$ & Page: 187 of 188 \\
PROGRAM PLAN & & & \\
\hline
\end{tabular}

\title{
Appendix D
}

\section{The Use of Nuclear Energy for Process Heat}

\author{
By J. Steve Herring
}

Nuclear reactors can and have been used to provide energy products other than electricity. Figure D-1 shows the approximate temperature ranges needed for a number of industrial thermal processes. For comparison, the reactor outlet temperatures used in electrical generation range from $300^{\circ} \mathrm{C}$ in light water or CANDU (Canadian Natural-Uranium, Heavy-Water-Moderated-and-Cooled Power) reactors to $950^{\circ} \mathrm{C}$ in very high temperature reactors (VHTRs) using helium as the coolant.

It is informative to consider the distribution of energy use in the U.S. economy. Overall, the United States consumes about 100 quadrillion BTU (quads; $100 \times 1015$ BTU) per year. The present fleet of 103 reactors annually produces about 8 quads. The electric power sector consumes a total of 40 quads annually, about half of which is supplied by coal. The transportation sector consumes 27 quads annually, almost entirely supplied by petroleum, $60 \%$ of which is imported. The residential sector consumes 5 quads of natural gas for home heating, while the commercial consumes 3 quads for building heating. By comparison, the industrial sector consumes about 9 quads of natural gas annually. While part of the natural gas use is for building heating, the majority is for process heat requirements, such as those shown above.

Thus the needs to which nuclear energy might readily be applied are first, the 20 quads of coal now being used for electricity generation, then the 5 quads of natural gas being used for industrial process heat and the 15 quads of petroleum being imported for the transportation sector.

In addressing our strategic vulnerabilities due to imported petroleum, it is important to recognize that the primary chemical difference between coal and petroleum is the amount of hydrogen in the chemical mixture. In simplified terms and ignoring impurities, coal is $\mathrm{CH} 0.8$ and petroleum is $\mathrm{CH} 1.8$. Thus, the most promising near-term use of nuclear heat and electricity will be for production of hydrogen for upgrading lower quality crude oils and oil sands. Later uses will be for the synthesis of conventional hydrocarbon fuels using nuclear-produced hydrogen and carbon from a variety of sources, initially coal, but later, sequestered $\mathrm{CO}_{2}$ and biomass. 


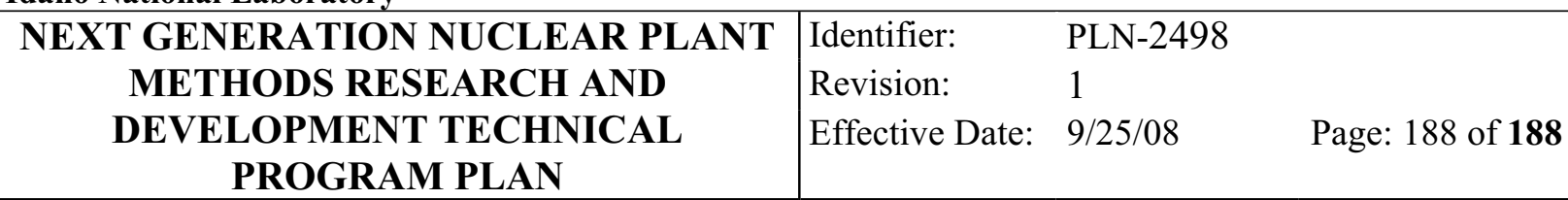

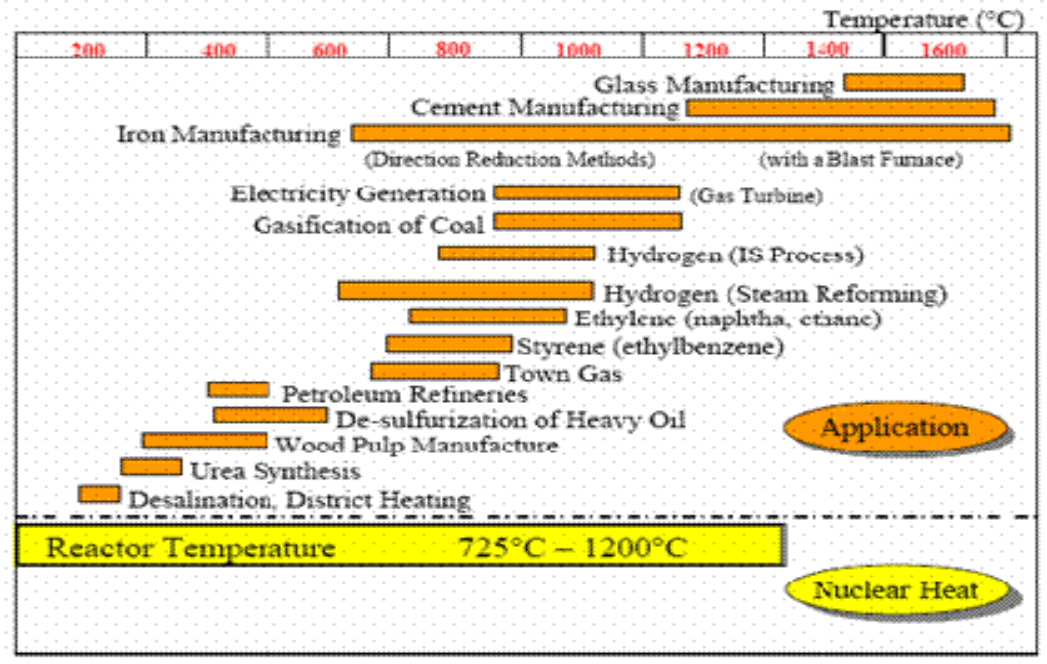

Figure D-1. Typical temperatures ranges needed by various industrial processes compared to design exit temperatures for an advanced VHTR. 